\title{
C02 Dissociation using the Versatile Atmospheric Dielectric Barrier Discharge Experiment (VADER)
}

\author{
Michael Allen Lindon \\ West Virginia University
}

Follow this and additional works at: https://researchrepository.wvu.edu/etd

\section{Recommended Citation}

Lindon, Michael Allen, "CO2 Dissociation using the Versatile Atmospheric Dielectric Barrier Discharge Experiment (VADER)" (2014). Graduate Theses, Dissertations, and Problem Reports. 285.

https://researchrepository.wvu.edu/etd/285

This Dissertation is protected by copyright and/or related rights. It has been brought to you by the The Research Repository @ WVU with permission from the rights-holder(s). You are free to use this Dissertation in any way that is permitted by the copyright and related rights legislation that applies to your use. For other uses you must obtain permission from the rights-holder(s) directly, unless additional rights are indicated by a Creative Commons license in the record and/ or on the work itself. This Dissertation has been accepted for inclusion in WVU Graduate Theses, Dissertations, and Problem Reports collection by an authorized administrator of The Research Repository @ WVU.

For more information, please contact researchrepository@mail.wvu.edu. 


\title{
$\mathrm{CO}_{2}$ Dissociation using the Versatile Atmospheric Dielectric Barrier Discharge Experiment (VADER)
}

\author{
by \\ Michael Allen Lindon \\ Dissertation submitted \\ to the Eberly College of Arts and Sciences \\ at West Virginia University \\ in partial fulfillment of the requirements for the degree of \\ Doctor of PhILOSOPhY in \\ PHYsics \\ Committee Members \\ Earl Scime, Ph.D., Chair \\ Paul Cassak, Ph.D. \\ Arthur Weldon, Ph.D. \\ D.J. Pisano, Ph.D. \\ Fred King, Ph.D. \\ Department of Physics \\ Morgantown, West Virginia \\ 2014
}

Keywords: $\mathrm{CO}_{2}$ Dissociation, Dielectric Barrier Discharge, DBD, Atmospheric Plasmas, Stark Broadening Spectroscopy, Plasma Chemistry, Plasma Chemical Model Copyright 2014 Michael Lindon 


\section{Abstract \\ $\mathrm{CO}_{2}$ Dissociation using the Versatile Atmospheric Dielectric Barrier Discharge Experiment (VADER)}

\section{Michael Allen Lindon}

As of 2013, the Carbon Dioxide Information Analysis Center (CDIAC) estimates that the world emits approximately 36 trillion metric tons of Carbon Dioxide $\left(\mathrm{CO}_{2}\right)$ into the atmosphere every year [1]. These large emissions have been correlated to global warming trends that have many consequences across the globe, including glacial retraction, ocean acidification and increased severity of weather events. With green technologies still in the infancy stage, it can be expected that $\mathrm{CO}_{2}$ emissions will stay this way for along time to come. Approximately $41 \%$ of the emissions are due to electricity production, which pump out condensed forms of $\mathrm{CO}_{2}$ [2]. This danger to our world is why research towards new and innovative ways of controlling $\mathrm{CO}_{2}$ emissions from these large sources is necessary.

As of now, research is focused on two primary methods of $\mathrm{CO}_{2}$ reduction from condensed $\mathrm{CO}_{2}$ emission sources (like fossil fuel power plants): Carbon Capture and Sequestration (CCS) and Carbon Capture and Utilization (CCU). CCS is the process of collecting $\mathrm{CO}_{2}$ using absorbers or chemicals, extracting the gas from those absorbers and finally pumping the gas into reservoirs. $\mathrm{CCU}$ on the other hand, is the process of reacting $\mathrm{CO}_{2}$ to form value added chemicals, which can then be recycled or stored chemically.

A Dielectric Barrier discharge (DBD) is a pulsed, low temperature, non-thermal, atmospheric pressure plasma which creates high energy electrons suitable for dissociating $\mathrm{CO}_{2}$ into its components ( $\mathrm{CO}$ and $\mathrm{O}$ ) as one step in the CCU process. Here I discuss the viability of using a DBD for $\mathrm{CO}_{2}$ dissociation on an industrial scale as well as the fundamental physics and chemistry of a $\mathrm{DBD}$ for $\mathrm{CO}_{2}$ dissociation. This work involved modeling the DBD discharge and chemistry, which showed that there are specific chemical pathways and plasma parameters that can be adjusted to improve the $\mathrm{CO}_{2}$ reaction efficiencies and rates.

Experimental studies using the Versatile Atmospheric dielectric barrier Discharge ExpeRiment (VADER) demonstrated how different factors, like voltage, frequency and the addition of a photocatalyst, change the efficiency of $\mathrm{CO}_{2}$ dissociation in VADER and the plasma chemistry involved. 


\section{Acknowledgments}

The process for getting a PhD was grueling, so I would like to thank everyone who helped me make this happen: My advisor, Dr. Earl Scime, for always being there to answer a question, for doing untold amounts of edits, and for putting up with my sass. The WVU physics department, especially my committee members (Dr. Earl Scime, Dr. Paul Cassak, Dr. H.A. Weldon, Dr. Fred King, and Dr. D.J. Pisano), for all of their hard work which made me the physicist I am today. Dr. Dushyant Shekhawat and those at NETL who put their faith in me and allowed my PhD project to take off. Dr. Michael Gallagher for being a great friend and colleague. My family for always being there to give advice and for keeping my connection between me and Chicago strong. Samantha Brooks for her constant encouragement and for always being there for me after a rough day; I never would have made it this far without you. 


\section{Contents}

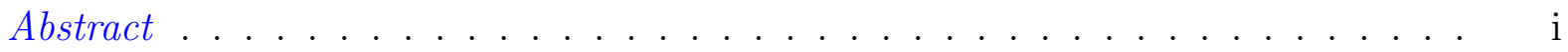

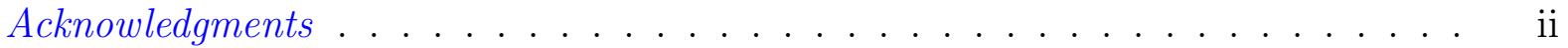

Table of Contents.................................... ii

List of Figures . . . . . . . . . . . . . . . . . . . . . . vi

1 Introduction $\quad 1$

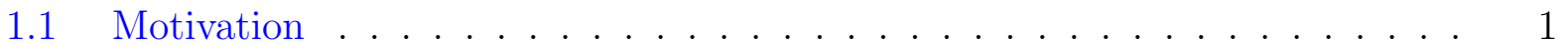

1.2 Research Goals . . . . . . . . . . . . . . . . . . . 3

1.3 The Research ....................... 4

2 Dielectric Barrier Discharge and $\mathrm{CO}_{2}$ Dissociation Theory 6

$2.1 \mathrm{CO}_{2}$ Dissociation Chemistry ..................... 6

2.1.1 $\mathrm{CO}_{2}$ Dissociation Energies . . . . . . . . . . . 7

2.1.2 Chemical Inefficiencies . . . . . . . . . . . . . . 8

2.1.3 Activation Energies and Catalysts . . . . . . . . . . . . 13

2.1.4 Free Radical Chemistry . . . . . . . . . . . . . . 16

2.2 Plasma Systems . . . . . . . . . . . . . . . . . . . . . . . . . . . . . 19

2.2.1 Atmospheric Pressure Plasmas . . . . . . . . . . . . . . . 20

2.2.2 Creating Atmospheric Plasmas . . . . . . . . . . . . . . . 21

2.2.3 LTE vs. Non-LTE Plasmas . . . . . . . . . . . . . . . . . 22 
2.2.4 Atmospheric Plasma Systems . . . . . . . . . . . . . . . 23

2.2.5 The Dielectric Barrier Discharge (DBD) . . . . . . . . . . . . 24

2.2.6 DBD Plasma Dynamics . . . . . . . . . . . . . . . 31

2.3 DBD Plasma Chemistry Model . . . . . . . . . . . . . . . . . . . . 44

2.3.1 The Cascade Phase . . . . . . . . . . . . . . . . . 45

2.3.2 The Afterglow Phase and Neutral Phase . . . . . . . . . . . . . 47

2.3.3 Pure $\mathrm{CO}_{2}$ Plasma Results . . . . . . . . . . . . . . . . . . . 49

2.3.4 $95 \% \mathrm{CO}_{2} / 5 \%$ CO Plasma Results ............. 55

2.3.5 $60 \% \mathrm{CO}_{2} / 40 \%$ Ar Plasma Results ............... 60

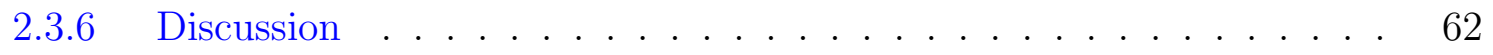

3 VADER Design $\quad 64$

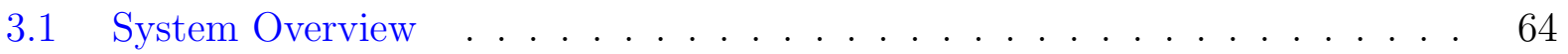

3.1.1 Reaction Chamber .................. 67

3.1.2 High Voltage $(\mathrm{HV})$ System . . . . . . . . . . . . . . 67

3.1.3 The Reaction Box ................... 73

3.1.4 Catalyst Mounting . . . . . . . . . . . . . 77

3.1.5 Gas System . . . . . . . . . . . . . . 78

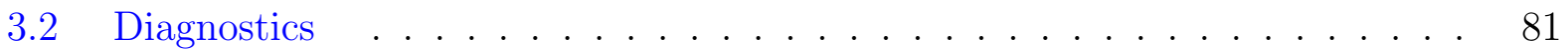

$3.2 .1 \quad$ RGA . . . . . . . . . . . . . . . . . . 82

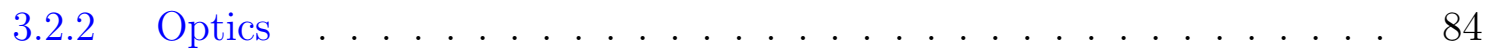

3.2.3 Power Measurements ................... 86

4 Optical Diagnostics in VADER $\quad 88$

4.1 Molecular Band Spectroscopy _. . . . . . . . . . . . . . . 89 
4.2 Stark Broadening Spectroscopy _. . . . . . . . . . . . . . 92

5 VADER $\mathrm{CO}_{2}$ Dissociation Experiments 106

5.1 Dielectric Tests . . . . . . . . . . . . . . . . 107

$5.2 \quad \mathrm{CO}_{2}$ Dissociation Experimental Setup . . . . . . . . . . . . 114

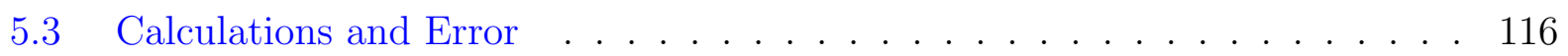

5.3.1 Energy Efficiency, Dissociation Rate and Percent Difference . . . . . 116

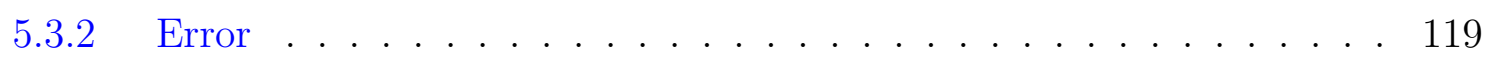

5.4 Experimental Results . . . . . . . . . . . . . . . . 121

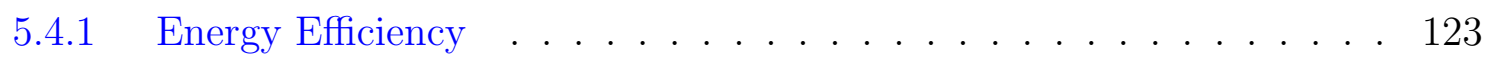

5.4 .2 Dissociation Rate .................... 126

5.4 .3 Gas Flow Rate . . . . . . . . . . . . . . . . 129

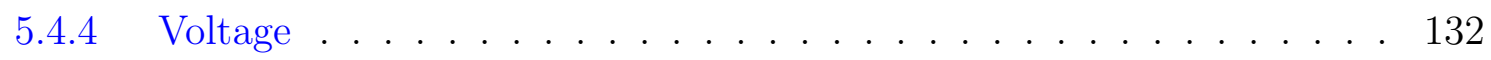

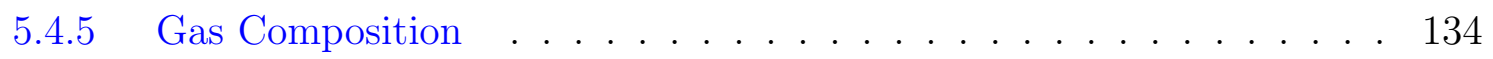

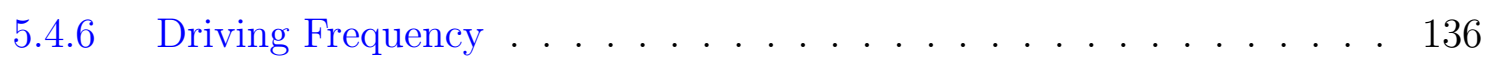

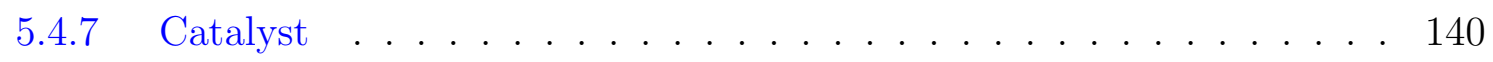

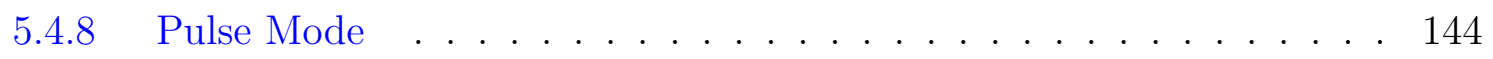

6 Summary 146

A Dielectric Material Analysis 153

A.1 First Dielectric Test . . . . . . . . . . . . . . . . . 153

A.2 Second Dielectric Test . . . . . . . . . . . . . . . . 154

B Collisional Cross-Sections for $\mathrm{CO}_{2}$ Dissociation 166 


\section{List of Figures}

2.1 Equilibrium Composition of $\mathrm{CO}_{2}$ in a Thermal System . . . . . . . . . 9

2.2 Electron $-\mathrm{CO}_{2}$ Cross-Sections . . . . . . . . . . . . . . . 14

2.3 DBD Designs . . . . . . . . . . . . . . . . . . . . . 27

2.4 Illustration of Streamer Formation . . . . . . . . . . . . . . . . . . 28

2.5 Anatomy of a Streamer . . . . . . . . . . . . . . . . . . . . . . . 29

2.6 Ion and Electron Cross-Sections . . . . . . . . . . . . . . 36

2.7 Ion and Electron Collision Frequencies . . . . . . . . . . . . . 37

2.8 Average Energy Gain of Ions and Electrons in an Electric Field . . . . . . . 38

2.9 Druyvesteyn vs. Maxwellian . . . . . . . . . . . . . . . . . . . . 43

2.10 Cascade Phase Reaction Rates - $100 \% \mathrm{CO}_{2}$. . . . . . . . . . . . . . 52

2.11 Afterglow/Neutral Phase Reaction Rates - $100 \% \mathrm{CO}_{2} \ldots \ldots$. . . . . . 54

2.12 Cascade Phase Reaction Rates - 92.5\% $\mathrm{CO}_{2}, 5 \% \mathrm{CO}, 2.5 \% \mathrm{O}_{2} \ldots \ldots 7$

2.13 Afterglow/Neutral Phase Reaction Rates - 92.5\% $\mathrm{CO}_{2}, 5 \% \mathrm{CO}, 2.5 \% \mathrm{O}_{2} \quad$. $\quad 59$

2.14 Cascade Phase Reaction Rates - $60 \% \mathrm{CO}_{2}, 40 \% \mathrm{Ar}, 3 \% \mathrm{CO}, 1.5 \% \mathrm{O}_{2}$. . . 61

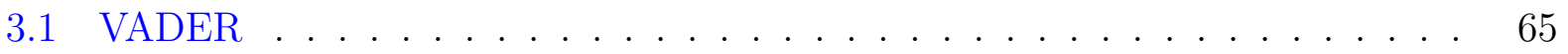

3.2 VADER Block Diagram . . . . . . . . . . . . . . . . 66

3.3 VADER Reaction Chamber Image . . . . . . . . . . . . . . . . . . 67

3.4 Voltage Drop vs. Frequency TREK Power Supply . . . . . . . . . . . . . . 69 
3.5 Example of Current, Voltage, and Power Graphs in VADER Using the DIDRIV10 Power Supply . . . . . . . . . . . . . . . . . . . . 70

3.6 Example Current-Voltage and Power Curves for Two Different Duty Cycles Using the DIDRIV10 Power Supply . . . . . . . . . . . . . . . 71

3.7 The HV Feedthrough Flange . . . . . . . . . . . . . . . . 73

3.8 Reaction Box Design . . . . . . . . . . . . . . . . . . 74

3.9 Reaction Box . . . . . . . . . . . . . . . . . . 75

3.10 Image of the Dielectric with Catalyst . . . . . . . . . . . . . . 77

3.11 Diagram of the VADER Gas System . . . . . . . . . . . . . . . 79

3.12 Picture of the VADER Heating Coil . . . . . . . . . . . . . . . . . 81

3.13 RGA Readout . . . . . . . . . . . . . . . . . . 84

3.14 Diagram of the VADER Focusing Optics . . . . . . . . . . . . . . 85

3.15 Picture of the VADER Voltage Divider . . . . . . . . . . . . . . 87

4.1 CO Molecular Band Structure in VADER $\mathrm{CO}_{2}$ Plasma . . . . . . . . . . 90

4.2 Molecular Band Structure of VADER Compared to Work by C. Rond [39] 91

4.3 Deconvolution Results for the 794.8176 nm Neutral Argon Line . . . . . . . 104

5.1 399.5nm Line Amplitudes When Using Alumina and Quartz Dielectric . . 112

5.2 TREK Energy Efficiency . . . . . . . . . . . . . . . . . . . . . 124

5.3 DIDRIV10 Energy Efficiency . . . . . . . . . . . . . . . . 125

5.4 Energy Efficiency Versus Power . . . . . . . . . . . . . . . . . 126

5.5 TREK Dissociation Rates . . . . . . . . . . . . . . . . . . 127

5.6 DIDRIV10 $\mathrm{CO}_{2}$ Dissociation Rates . . . . . . . . . . . . 128

$5.7 \mathrm{CO}_{2}$ Dissociation Rate Versus Power . . . . . . . . . . . . . . 130 
5.8 Percent Difference - Gas Flow Rate. . . . . . . . . . . . . . . . . . . . . . 131

5.9 Percent Difference - Voltage . . . . . . . . . . . . . . . . 133

5.10 Percent Difference - Gas Composition . . . . . . . . . . . . . . 135

5.11 Percent Difference in Forward Power Between Gas Compositions for the DIDRIV10 Power Supply . . . . . . . . . . . . . . . . . . . . 136

5.12 Energy Efficiency Versus Frequency . . . . . . . . . . . . . . . 137

5.13 Percent Difference - Power Supply Driving Frequency . . . . . . . . . . . . 138

5.14 Percent Difference - Power Supply Driving Frequency Separated by Gas Composition and Voltage . . . . . . . . . . . . . . . . 139

5.15 Effects of Lower Breakdown Voltages and Higher Applied Voltages on a DBD 141

5.16 Percent Difference - Catalyst . . . . . . . . . . . . . . . . . . 142

5.17 Percent Difference in Forward Power due to the Addition of P25 $\mathrm{TiO}_{2}$ into the TREK Power Supply . . . . . . . . . . . . . . . . . . . . 143

5.18 Percent Difference in Forward Power Due to the Addition of P25 $\mathrm{TiO}_{2}$ into the DIDRIV10 Power Supply . . . . . . . . . . . . . . . . 143

5.19 Percent Difference - Frequency . . . . . . . . . . . . . . . . . . 145 


\section{Chapter 1}

\section{Introduction}

\subsection{Motivation}

One of the biggest issues in our modern day is the effect of global warming and the destructive consequences it has on the global ecosystem. Evidence points to increased amounts of greenhouse gas emissions by humans as the main cause [3]. Greenhouse gases work to keep the Earth warm by trapping large portions of the radiation of the Sun on Earth. Therefore, the density of greenhouse gases in the Earth's atmosphere is directly related to the temperature of the Earth's surface. Some of the consequences of higher densities of greenhouse gases and higher surface temperatures consist of acidification of oceans due to increased carbon dioxide $\left(\mathrm{CO}_{2}\right)$ absorption, the melting of the ice caps, and a global increase in severe weather due to larger gradients in temperature [4]. These consequences lead to many issues worldwide, including the destruction of animal habitats, flooding of cities, and higher rates of global desertification. The majority of greenhouse gases supporting the Earth are water vapor $\left(\mathrm{H}_{2} \mathrm{O}\right), \mathrm{CO}_{2}$, methane $\left(\mathrm{CH}_{4}\right)$, nitrous oxide $\left(\mathrm{N}_{2} \mathrm{O}\right)$ and ozone $\left(\mathrm{O}_{3}\right)$. A substantial portion of the current temperature trends $(>60 \%)$ can be correlated to increased $\mathrm{CO}_{2}$ emission due to 
the industrialization of countries and $90 \%$ of $\mathrm{CO}_{2}$ emissions come from the burning of fossil fuels $[5]$.

Efforts to reduce the use of fossil fuels have led to several approaches for reducing $\mathrm{CO}_{2}$ emissions. For instance, in the US there has been a strong push towards more fuel-efficient vehicles, electric cars, energy efficient appliances, and green electricity (i.e. solar and wind power). These reduction methods have reduced the $\mathrm{CO}_{2}$ emissions from vehicles in the US (which create $28 \%$ of $\mathrm{CO}_{2}$ emission) and electricity demands from fossil fuel power plants (33\% of $\mathrm{CO}_{2}$ emissions) [6]. However, as of 2011 the world produced over $80 \%$ of its electricity from fossil fuels and demand for power has continued to rise as more nations have industrialized [2]. This means that unless a new form of power is found in the near future, the world will still require power plants that pump trillions of metric tons of $\mathrm{CO}_{2}$ into the atmosphere. This motivates research into the reduction of $\mathrm{CO}_{2}$ emissions from power plants.

Currently, there are two proposed methods for reducing $\mathrm{CO}_{2}$ emissions from power plants, the most popular of which is carbon capture and sequestration (CCS) followed by carbon capture and utilization $(\mathrm{CCU})$. CCS is the process of collecting $\mathrm{CO}_{2}$ by separating the $\mathrm{CO}_{2}$ from the flue gas of a reactor or power plant using $\mathrm{CO}_{2}$ absorbers or chemical processes, followed by transporting the gas to a reservoir to be permanently stored [7]. The proposed storage locations are either underground or in mineral deposits. CCS entails many complications, including finding a suitable place to act as a reservoir while avoiding harm to the surrounding environment and being able to permanently contain the gas. Sites with such properties are difficult to find and the increased energy costs of separating, transporting, and pumping the $\mathrm{CO}_{2}$ gas into its final location puts a large limitation on the efficacy of carbon sequestration [8]. Even so, large amounts of research have and are currently being done to overcome these issues. 
$\mathrm{CCU}$ involves converting $\mathrm{CO}_{2}$ into different chemicals that can be stored or used commercially. Research in this method of $\mathrm{CO}_{2}$ remediation has taken many forms, including growing algae using the flue gas of power plants, then harvesting the algae as biofuel and converting $\mathrm{CO}_{2}$ directly into hydrocarbons or commercial products using different combinations of heat, pressure, catalysts and plasma $[9,10]$. Each of these methods come with their own set of issues, which most often stem from low energy efficiencies and chemical selectivity issues.

It is clear that these $\mathrm{CO}_{2}$ removal methods need to be further researched if we are to overcome these issues in the future. This work focuses on the efficacy of atmospheric plasmas for CCU, specifically the Dielectric Barrier Discharge (DBD), and photocatalysts for the dissociation of $\mathrm{CO}_{2}$ into its constituent parts $\left(\mathrm{CO}\right.$ and $\left.\mathrm{O}_{2}\right)$ in the hopes of converting it into value added chemicals.

\subsection{Research Goals}

Atmospheric plasmas have been used for many things over the years, from ozone production, to plasma TVs, to electric arc furnaces, and the destruction of hazardous waste. [11-14] The usefulness of atmospheric plasmas is due to their ability to non-thermally excite high energy chemistry unavailable to traditional heating methods at large scales. The ability of nonthermal plasmas to excite these chemistries has created interest in using them for CCU. Some of the plasma systems previously tested are dielectric barrier discharges (DBDs) [15-21], microwave plasmas $[22,23]$, radio frequency $(\mathrm{RF})$ plasmas $[24,25]$ along with several other unique systems [26-32]. The biggest issue with current research in plasma CCU is that the plasma, in most cases, is treated only as an energy source for reactions. While this 
treatment can be effective for thermal plasmas, as there is little difference between a thermal plasma and a high temperature system, it is not appropriate when dealing with non-thermal plasmas (which distribute energy unevenly between molecular states), like DBDs and corona discharges. It is this uneven distribution of energy, which allows for the activation and deactivation of specific reaction pathways, that makes plasma CCU unique. Therefore, this work looks to understand the relationship between the dynamics of non-thermal plasmas and the $\mathrm{CO}_{2}$ chemistry in order to improve the current research into plasma CCU.

\subsection{The Research}

The first step to $\mathrm{CCU}$ is the highly endothermic process of $\mathrm{CO}_{2}$ dissociation $(\geq 5.52 \mathrm{eV} /$ molec) into its constituent parts ( $\mathrm{CO}$ and $\mathrm{O}$ ), which requires the high energy, non-thermal processes of a plasma. Chapter 2 reviews the dissociation chemistry and plasma physics important for $\mathrm{CO}_{2}$ dissociation. The results of this analysis showed that, of the atmospheric plasmas available, the $\mathrm{DBD}$ is the most appropriate plasma for $\mathrm{CO}_{2}$ dissociation. A DBD is an atmospheric pressure, non-thermal $\left(T_{e} \gg T_{i}\right)$, low temperature plasma formed of a collection of short-lived, filamentary discharges with typical electron temperatures of 1-10 $\mathrm{eV}$, plasma densities in the range of $10^{14}-10^{15} \mathrm{~cm}^{-3}$ and scalability appropriate for use in a commercial setting. The electron and ion energies within the discharge were calculated in order to understand the limitations of the DBD. The particle energies were then applied to a chemical model of the DBD discharge, which was used to determine the limiting chemical reactions of the plasma.

The plasma chemistry led to the design of the Versatile Atmospheric Discharge ExpeRiment (VADER), detailed in Chapter 3. VADER was built with the flexibility to test 
multiple gas flows, dielectrics, gas mixtures, catalysts, pressures, frequencies, voltages, discharge gaps, and waveforms. The chemical sampling system consisted of an RGA, which continuously sampled the reacted gases through a set of capillary leak valves, and power measurements (voltmeter, voltage divider) to determine the efficiency and dissociation rate of the discharge. Immersion probes are not possible in a DBD due to the high voltages involved, hence for plasma diagnostics two optical breadboards were built into the VADER test stand to allow for both injection and collection optics.

Chapter 4 discusses the results of the optical diagnostics used in VADER. The diagnostic methods included molecular band spectroscopy (which was used as the first sign of dissociation within the plasma) and Stark broadening spectroscopy (to look into the electron temperature of the plasma). Due to non-ideal broadening in the DBD, Stark broadening was used to verify the gas temperature of the VADER discharge.

Chapter 5 details the $\mathrm{CO}_{2}$ dissociation experiments in VADER. The first variable tested was the DBD dielectric. Several materials were tested for their power coupling and discharge physics in order to determine the material best suited for the dissociation experiments. Using the dielectric results, the $\mathrm{CO}_{2}$ dissociation experiments were conducted. The variables tested during the dissociation experiments included the gas flow rate, the power supply voltage and frequency, gas composition, the effects of adding a photocatalyst, and multiple pulse modes. One of the main discoveries was a resonant driving frequency at which $\mathrm{CO}_{2}$ dissociation is most efficient and how it is affected by the breakdown properties of the gas (dictated by the voltage and gas composition). Another important discovery was an improvement in dissociation efficiency and rate with the introduction of certain gases and the inclusion of a photocatalyst into the DBD. 


\section{Chapter 2}

\section{Dielectric Barrier Discharge and $\mathrm{CO}_{2}$ Dissociation Theory}

The dissociation of carbon dioxide $\left(\mathrm{CO}_{2}\right)$ within a plasma involves a complex relationship between the chemistry of $\mathrm{CO}_{2}$ and the dynamics of the plasma. For efficient dissociation the plasma needs to excite specific molecular energy states to overcome the bond energies of $\mathrm{CO}_{2}$ and avoid many of the energy loss mechanisms, like inefficient energy use and reverse reactions. Through modeling and analysis, it is shown that the electron cascade of a dielectric barrier discharge (DBD) is well-suited for the dissociation of $\mathrm{CO}_{2}$, which creates the radicals needed for the formation of value-added chemicals. However, the relaxation of those radicals is what truly determines the efficiency of these discharges.

\section{1 $\quad \mathrm{CO}_{2}$ Dissociation Chemistry}

To find the most efficient reaction pathways within VADER and to motivate the use of plasmas for $\mathrm{CO}_{2}$ dissociation, the full chemical thermodynamics of $\mathrm{CO}_{2}$ dissociation must 
be understood. To simplify the chemistry in VADER, $\mathrm{CO}_{2}$ was the only active, chemical species inserted into the plasma (argon does not readily form molecular bonds). Even so, the chemistry for $\mathrm{CO}_{2}$ dissociation is complicated, especially when taking into account the various plasma dynamics.

\subsection{1 $\quad \mathrm{CO}_{2}$ Dissociation Energies}

The dissociation chemistry of $\mathrm{CO}_{2}$ in a pure $\mathrm{CO}_{2}$ environment is as follows [33]:

$$
\mathrm{CO}_{2}+\text { heat } \longrightarrow \mathrm{CO}^{*}+\mathrm{O} \quad \Delta H \sim 532.2 \frac{\mathrm{kJ}}{\mathrm{mol}}=8.38 \mathrm{eV}
$$

$\mathrm{CO}^{*}$ is carbon monoxide with a double bond, which then relaxes to form a triple bond

$$
C O^{*} \longrightarrow C O \quad \Delta H \sim 532.2 \frac{\mathrm{kJ}}{\mathrm{mol}}=-2.86 \mathrm{eV}
$$

The oxygen atom $(\mathrm{O})$ reacts with other oxygen atoms or $\mathrm{CO}_{2}$ to form $\mathrm{O}_{2}$

$$
2 O \longrightarrow \mathrm{O}_{2} \quad \Delta H \sim 32.8 \frac{\mathrm{kJ}}{\mathrm{mol}}=-5.16 \mathrm{eV}
$$

or

$$
\mathrm{O}+\mathrm{CO}_{2} \longrightarrow \mathrm{O}_{2}+\mathrm{CO} \quad \Delta H \sim 32.8 \frac{\mathrm{kJ}}{\mathrm{mol}}=0.34 \mathrm{eV}
$$

Combining the above equations gives the full reaction [34]:

$$
\mathrm{CO}_{2} \longrightarrow \mathrm{CO}+\frac{1}{2} \mathrm{O}_{2} \quad \Delta \mathrm{H} \sim 283.3 \frac{\mathrm{kJ}}{\mathrm{mol}}=2.94 \mathrm{eV}
$$


Activation energy is the minimum input energy needed to start a reaction, in this case the dissociation of $1 \mathrm{CO}_{2}$ molecule. Based on the Arrhenius equations, the activation energy for $\mathrm{CO}_{2}$ dissociation is $\sim 5.5 \mathrm{eV}$, which is approximately the combined energies of equations 2.1 and $2.2[14,35]$. These similar energies mean that equations 2.1 and 2.2 occur simultaneously during the dissociative process and are often represented by the combined equation

$$
\mathrm{CO}_{2}+\text { heat } \rightarrow \mathrm{CO}+\mathrm{O} \quad \Delta H \sim 532.2 \frac{\mathrm{kJ}}{\mathrm{mol}}=5.52 \mathrm{eV} .
$$

After this initial dissociative process, either $2.58 \mathrm{eV}$ of input energy per $\mathrm{O}$ is recovered through oxygen recombination or an additional $0.34 \mathrm{eV}$ is spent to remove an oxygen from a $\mathrm{CO}_{2}$ molecule, easily attainable in a plasma. Therefore, the minimum energy cost for dissociating a single $\mathrm{CO}_{2}$ molecule into carbon monoxide $(\mathrm{CO})$ and oxygen $\left(\mathrm{O}_{2}\right)$ is $2.94 \mathrm{eV}$. However, the above chemical equations only cover the energy for the most basic dissociation path and do not take into account the many single and multistep reaction paths that occur within a $\mathrm{CO}_{2}$ plasma, nor any chemical and system inefficiencies $[14,36]$.

\subsubsection{Chemical Inefficiencies}

Quenching refers to the rate at which reactants are cooled in an effort to halt reverse reactions and can be done a number of ways, including spraying cold particles into the heated gas and using heat exchangers. In the case of thermal $\mathrm{CO}_{2}$ decomposition, temperatures between $3000-5000 \mathrm{~K}$ are needed to effectively dissociate $\mathrm{CO}_{2}$ at atmospheric pressure-see Figure 2.1 [27]. Heating a reactor to these temperatures requires an immense amount of energy (which is not economically viable) and if the system isn't properly quenched, reverse reactions will dominate the system kinetics as the gas cools [14]. The reverse reaction is the conversion 
of $\mathrm{CO}$ and $\mathrm{O}_{2}$ back into $\mathrm{CO}_{2}$.

$$
\mathrm{CO}+\frac{1}{2} \mathrm{O}_{2} \longrightarrow \mathrm{CO}_{2} \quad \Delta H \sim-283.26 \frac{\mathrm{kcal}}{\mathrm{mol}}=-2.94 \frac{\mathrm{eV}}{\mathrm{molec}}
$$

The best way to combat this inefficiency is to either use a system with a very large quenching rate or use a low temperature system, like a DBD.

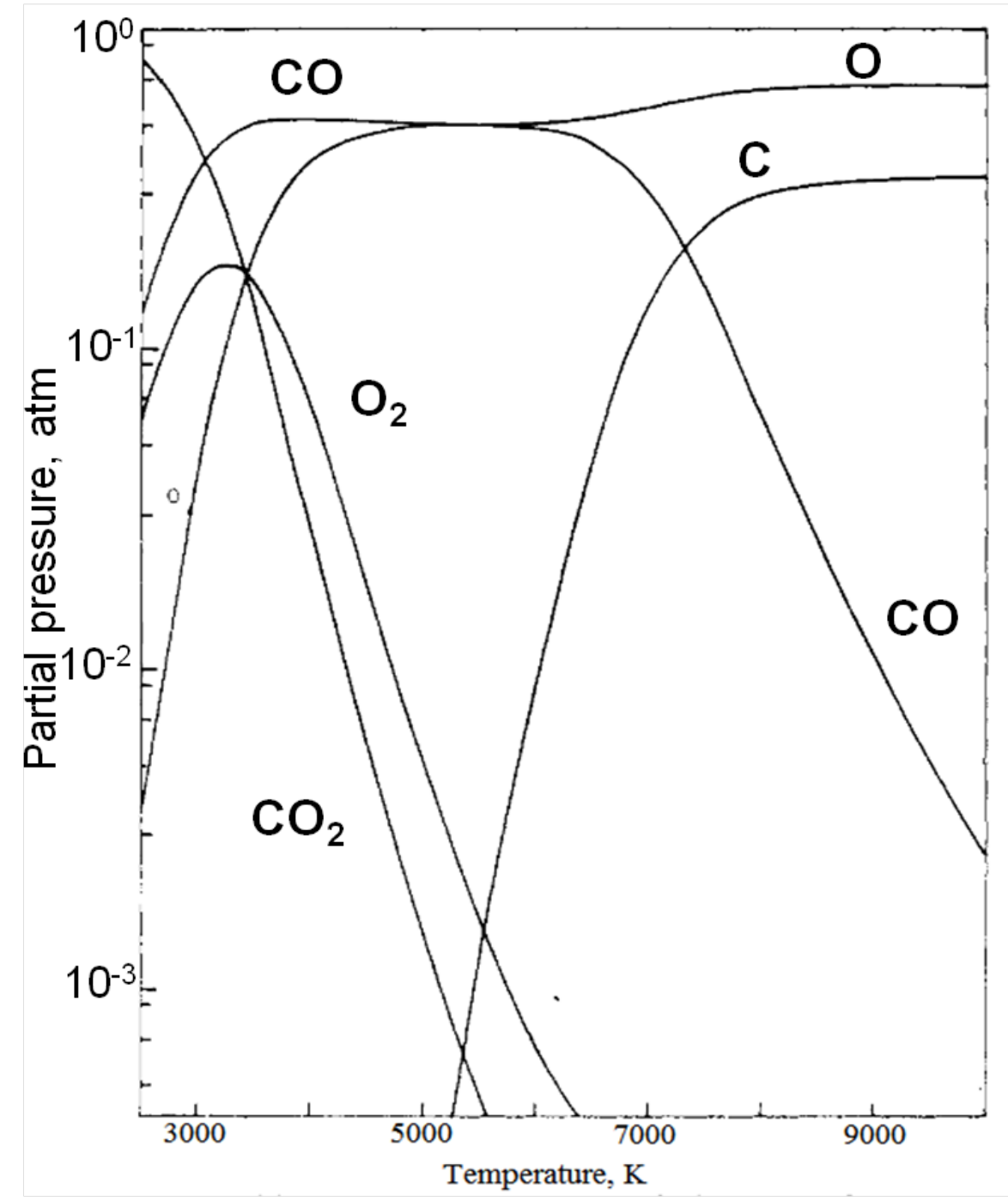

Figure 2.1: The equilibrium composition of $\mathrm{CO}_{2}$ and its constituents in a thermal plasma reactor at $1 \mathrm{~atm} .[27]$ 
The quenching process is linked to energy recuperation. Energy recuperation is the ability of a system to reuse the input and reaction energies of a chemical system for other purposes. For example, running the exhaust gas through a heat exchanger to preheat the reaction gas and encourage endothermic reactions, like $\mathrm{CO}_{2}$ reduction (Equation 2.4). In the case of $\mathrm{CO}_{2}$ dissociation, no recuperation would be equivalent to losing most of the $2.86 \mathrm{eV}$ recovered when the $\mathrm{CO}$ double bonds form a triple bonds and the $5.16 \mathrm{eV}$ of energy recovered during oxygen atom recombination (Equation 2.3), which increases the dissociation energy cost by $5.44 \mathrm{eV}$ per $\mathrm{CO}_{2}$ dissociated. For most plasmas, ionization is an energy intensive task $(13.8 \mathrm{eV})$, so it is important to recover the electron energy from this process. Therefore, a viable system for $\mathrm{CO}_{2}$ dissociation must be able to incorporate some form of energy recuperation. In the VADER experiments, energy recuperation was not addressed and therefore $\mathrm{CO}_{2}$ dissociation in VADER was expected to require much greater energies than $2.94 \mathrm{eV}$ per reaction.

For efficient dissociation, it is important that energy is applied where it is needed. In a molecule there are four major reservoirs into which energy can be deposited: the vibrational, electronic, rotational and kinetic states. Each of these reservoirs enables a different chemical path for dissociation with a different efficiency. For example, by exciting the vibrational states of $\mathrm{CO}_{2}$ with a microwave discharge at moderate pressures (50-200 Torr) an energy efficiency of up to $90 \%$ was achieved [14]. The molecular states and their application towards $\mathrm{CO}_{2}$ dissociation paths are described below:

- Vibrational state - Energy stored in the bending, flexing, and vibrating chemical bonds.

- Energy in the vibrational state of $\mathrm{CO}_{2}$ puts stress on the $\mathrm{C}-\mathrm{O}$ bonds, which either break the bonds or make it easier for collisions to break the bonds. It is 
suggested that energy placed in the asymmetric vibrational mode (001, when the two C-O bonds stretch and compress oppositely) is the most efficient method of dissociation [14].

- Electronic state - Energy stored in free electrons and bound electron energy levels

- High energy electrons go through impact dissociation or recombination pathways that directly dissociate $\mathrm{CO}_{2}$ or excite vibrational states (same as above).

- Rotational state - Energy of a molecule rotating about its axes

- None, energy must be mode-converted to other states for use in $\mathrm{CO}_{2}$ dissociation

- Kinetic energy - The ballistic motion of the molecules

- High energy collisions can cause $\mathrm{CO}_{2}$ dissociation through mode conversion into vibrational states, but kinetic energy is also easily mode-converted into rotational and electronic state energy.

Rotational excitation and kinetic excitation are inefficient pathways for dissociation. Rotational excitation does not have a direct route for molecular dissociation and must be mode-converted to the other three energy states to be useful for dissociation. Therefore, rotational energy is considered an energy sink for the purposes of $\mathrm{CO}_{2}$ dissociation. Increasing the kinetic energy of molecules for the purposes of dissociation requires accelerating heavy particles to high speeds and having them collide with one another in the hopes of dissociation. Kinetic excitation is inefficient because kinetic energy is easily transferred to other energy states, and at the high pressures needed for $\mathrm{CO}_{2}$ dissociation (atmospheric pressure or higher) the collision rates are too high for efficient ion/neutral particle acceleration. 
The most efficient $\mathrm{CO}_{2}$ dissociation path puts all of its energy into vibrational excitation, while keeping kinetic, electronic and rotational energies at a minimum [14,33,37]. This is because the bending and compressing of molecular bonds caused by vibrational energy takes the most direct path to dissociation. Also, assuming the bending and compressing of molecular bonds isn't enough to break $\mathrm{CO}_{2}$ 's bonds, the stress on the molecular bonds makes it easier for low energy collisions to break the bonds. Direct excitation of vibrational modes can be accomplished using lasers in the infrared, optical and UV ranges. However, the efficiencies of lasers are often very low and there are many problems keeping optical windows clean during industrial processes. Such issues can be especially important when working with reactive substances like the carbon in $\mathrm{CO}_{2}$.

If direct vibrational excitation is not possible, electronic excitation is the next best dissociative process. See Figure 2.2 for a summary of all electron- $\mathrm{CO}_{2}$ cross-sections. Electron energy can be efficiently mode converted to vibrational energy. The electronic-vibrational cross-section for $\mathrm{CO}_{2}$ peaks at electron energies between 3-6 eV with a cross-section greater than $1 \times 10^{-16} \mathrm{~cm}^{2}$ for $T_{e}>1 \mathrm{eV}$ [38]. These coupling energies are comparable to the bond energies of $\mathrm{CO}_{2}$ and are within range of electron temperatures seen in atmospheric plasmas, thus making atmospheric plasmas a viable path to $\mathrm{CO}_{2}$ dissociation. Electronic excitation also allows for additional dissociation pathways, the most common of which are dissociative attachment, impact dissociation, and dissociative ionization. Each pathway has a different outcome when an energized electron attaches to a molecule and puts the molecule into an unstable state, which then decays through dissociation [39,40]. The main difference between 
the three processes is the required energy and the number of free electrons after dissociation.

$$
\begin{aligned}
A X+e^{-} \longrightarrow A X^{-*} & \longrightarrow A^{-}+X \quad \text { (dissociative attachment) } \\
& \longrightarrow A+X+e^{-} \quad \text { (impact dissociation) } \\
& \longrightarrow A+X^{+}+2 e^{-} \text {(dissociative ionization) }
\end{aligned}
$$

For $\mathrm{CO}_{2}$ reactions, $\mathrm{AX}$ is $\mathrm{CO}_{2}, \mathrm{~A}$ is $\mathrm{O}$, and $\mathrm{X}$ is $\mathrm{CO}$. The minimum energies required for impact dissociation and dissociative ionization in $\mathrm{CO}_{2}$ are $12 \mathrm{eV}$ and $25 \mathrm{eV}$, respectively [41]. These high energies are required because the electron must have enough energy to both cause dissociation and cause one or two electrons to overcome the molecule's, or its constituents' ionization potential $(13.8 \mathrm{eV})$ [42]. Because an electron gains energy as it falls through the potential well of the particle it binds to, dissociative attachment in $\mathrm{CO}_{2}\left(\rightarrow \mathrm{CO}+\mathrm{O}^{-}\right)$occurs at electron energies as low as $3.4 \mathrm{eV} .3 .4 \mathrm{eV}$ is much lower than the C-O binding energy, thus making it an effective method of dissociation in a DBD. However, the dissociative attachment cross-section $\left(\sigma_{\text {diss attach }}<5 \times 10^{-19} \mathrm{~cm}^{2}\right.$ for $\left.3.4<T_{e}<10 \mathrm{eV}\right)$ is a factor of $10^{3}$ lower than the electronic-vibrational excitation cross-section, resulting in a much slower rate than vibrational excitation $[38,43]$.

\subsubsection{Activation Energies and Catalysts}

All chemical processes require a minimum activation energy, above the molecule's bond energies, to start a reaction. The activation energy is dependent on the bond structure and the reaction pathways of the molecule being reacted. Depending on the pathways in use, the total energy input can be close to the sum of the binding energies of the molecules' or much larger. At the end of the reaction, the activation energy is usually returned to the 


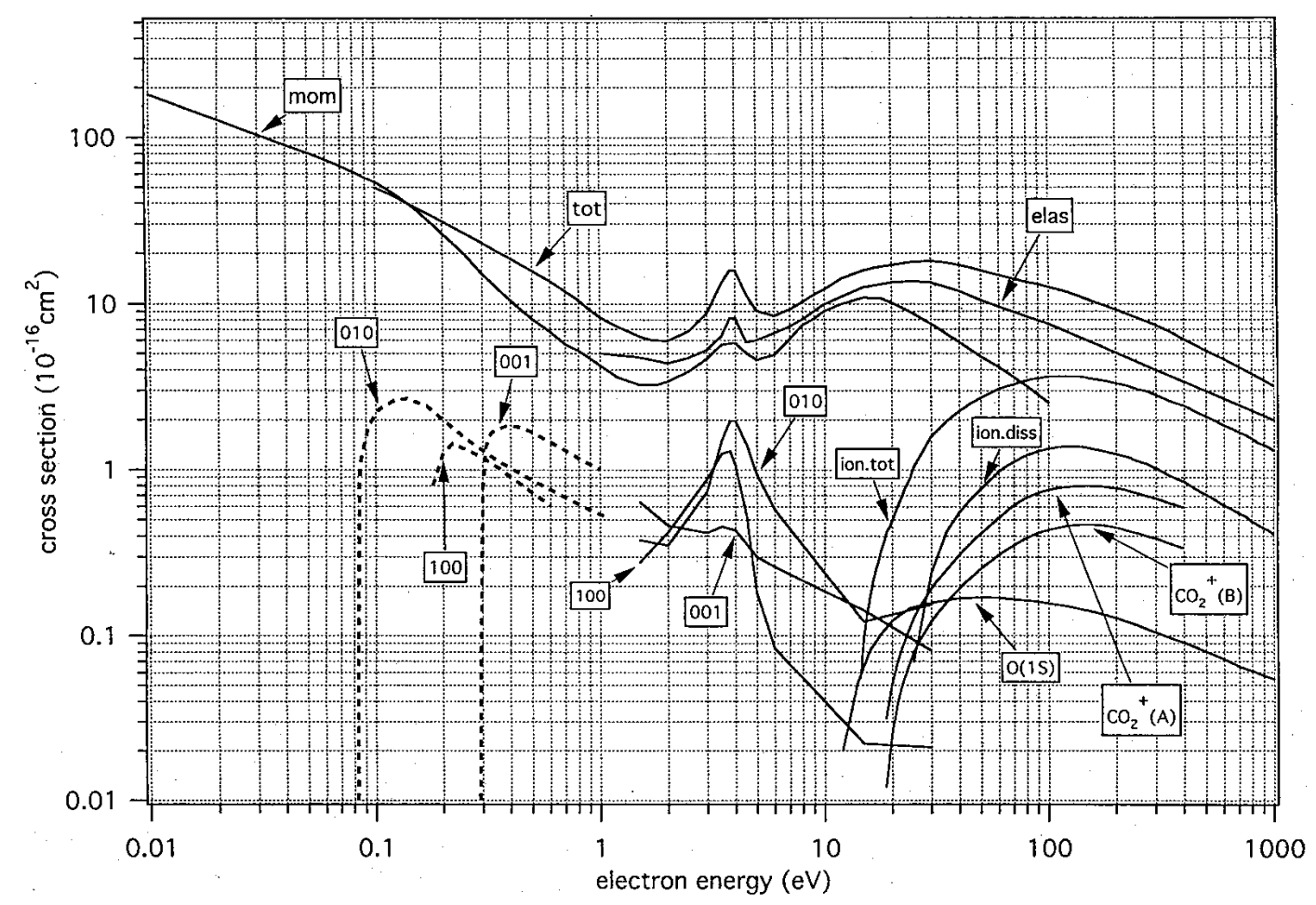

Figure 2.2: The electron - $\mathrm{CO}_{2}$ cross-sections. The three numbered cross-sections (010, 001, etc) are vibrational excitation cross-sections [38]

system as thermal energy. A catalyst is a chemical that, when introduced to a chemical reaction, provides an alternative reaction path without being consumed during the reaction. The alternate reaction path either decreases the activation energy for a specific reaction or increases the cross-section of the reaction by exposing bonds to collisions. These changes can increase the reaction rate, or, if the catalyst applies to the reverse reaction, slow down the reaction rate or even reverse it. For a catalytic reaction to occur, the catalyst needs to be in direct contact with the reactants. Therefore, the surface area of the catalyst is extremely important for most catalytic reactions. There are many methods for increasing the surface area of a catalyst, all of which depend on the form of the catalyst and its reactants. Examples of increasing the surface area of solid catalysts for use with gaseous reactants include applying the catalyst to a mesh grid, or pulverizing the catalyst and blowing it through the reactant 
stream.

The activation energy for $\mathrm{CO}_{2}$ dissociation is approximately $\sim 5.5 \mathrm{eV}$ equal to the bond energies of formation of $\mathrm{CO}$ and $\mathrm{O}[14,35]$. With such a small difference between activation energies and bond energies a traditional catalyst is ineffective. Therefore, a photocatalyst was used in these experiments. A photocatalyst is a material that becomes a catalyst only when it is energized above a certain energy. Any source of energy may be used to energize a photocatalyst, but it is most frequently accomplished through photoexcitation (shining a light of a specific energy on the material). The light/energy bridges the material's electron band gap, thus activating the chemical's catalytic properties [44]. The catalyst used in VADER was P25 $\mathrm{TiO}_{2}$, a mixture of $\mathrm{TiO}_{2}$ in the rutile and anatase phase. P25 $\mathrm{TiO}_{2}$ is currently used to disinfect water, decompose organic matter on windshields (self-cleaning glass), oxidize organic materials, and break down $\mathrm{CO}_{2}$ at room temperature when illuminated with light in the near UV (the reaction rate is extremely slow) [45-49]. Since $\mathrm{CO}_{2}$ has such strong bonds and $\mathrm{TiO}_{2}$ has been shown to dissociate $\mathrm{CO}_{2}$ at low temperatures $(300 \mathrm{~K})$, the photocatalyst activation energy must directly couple into $\mathrm{CO}_{2}$ 's molecular bonds, thus causing dissociation. Most plasmas naturally emit light $\left(\mathrm{CO}_{2}\right.$ has emission lines in the near UV) and have large energy losses to the walls of reactors through collisions. For these experiments, it is expected that the addition of a photocatalyst will allow for the energy loss due to these mechanisms to be coupled back into the dissociative process. One goal of these experiments was to determine if the light/particle energies of a DBD were sufficient to activate the $\mathrm{TiO}_{2}$ catalyst and then significantly effect the energy efficiency and rate of $\mathrm{CO}_{2}$ dissociation. 


\subsubsection{Free Radical Chemistry}

The previous sections discuss the methods and energy requirements for dissociating $\mathrm{CO}_{2}$ molecules into $\mathrm{CO}$ and $\mathrm{O}_{2}$. However, it is also important to discuss the chemistry of free radicals during dissociation. The relevant free radicals to be discussed herein are $\mathrm{O}, \mathrm{CO}_{2}^{+}$ and $\mathrm{Ar}^{+}$. Noble gases are known to reduce the break down voltage in plasmas and not change the chemistry, therefore tests were done to explore the effect of argon on the plasma chemistry of VADER. Other free radicals found during $\mathrm{CO}_{2}$ dissociation are $\mathrm{CO}_{2}^{-}, \mathrm{CO}^{+}$, $\mathrm{O}_{2}^{-}, \mathrm{O}^{+}$and $\mathrm{O}^{-}$; each has such a small population at atmospheric pressures such that their contributions to the dissociation chemistry are negligible.

\section{Atomic Oxygen (O)}

$\mathrm{O}$ is a byproduct of dissociating $\mathrm{CO}_{2}$ and, due to its high electronegativity, it quickly reacts with other molecules. In a $\mathrm{CO}_{2}$ plasma, $\mathrm{O}$ will follow one of four reaction pathways: $\mathrm{O}-\mathrm{O}$ bonding, $\mathrm{CO}_{2}$ reduction, $\mathrm{CO}$ oxidation, or ozone formation-see Table 2.1. O-O bonding and $\mathrm{CO}_{2}$ reduction create $\mathrm{O}_{2}$ the final $\mathrm{CO}_{2}$ dissociation product. Ozone at low temperatures decays into $\mathrm{O}_{2}$ or back into $\mathrm{O}$ at higher temperatures. $\mathrm{CO}$ oxidation is undesirable and creates $\mathrm{CO}_{2}$. Energetically, each of the $\mathrm{O}$ reactions is favorable, and the competition between these reactions is largely responsible for determining the final gas composition and efficiency of $\mathrm{CO}_{2}$ dissociation in both plasma and thermal environments.

Table 2.1: The most common atomic oxygen reactions in a $\mathrm{CO}_{2}$ plasma

\begin{tabular}{lll} 
Reaction & & $\Delta \mathrm{H}(\mathrm{eV})$ \\
\hline O-O Recombination & $2 \mathrm{O}+\mathrm{M} \rightleftharpoons \mathrm{O}_{2}+\mathrm{M}$ & -5.16 \\
$\mathrm{CO}_{2}$ Reduction & $\mathrm{O}+\mathrm{CO}_{2} \rightleftharpoons \mathrm{O}_{2}+\mathrm{CO}$ & +0.34 \\
CO Oxidation & $\mathrm{O}+\mathrm{CO}+\mathrm{M} \rightleftharpoons \mathrm{CO}_{2}+\mathrm{M}$ & -5.52 \\
Ozone Formation & $\mathrm{O}+\mathrm{O}_{2}+\mathrm{M} \rightleftharpoons \mathrm{O}_{3}+\mathrm{M}$ & -4.61
\end{tabular}




\section{$\mathrm{CO}_{2}^{+}$}

Due to the large energies needed for the ionization of $\mathrm{CO}_{2}(13.8 \mathrm{eV})$, significant populations of $\mathrm{CO}_{2}^{+}$are almost exclusively found within plasmas. $\mathrm{CO}_{2}^{+}$is formed through the collision of $\mathrm{CO}_{2}$ with electrons, photons, and ions/neutral particles, or a combination such that they excite electrons above their ionization energies [50]. When this excitation occurs due to electron collisions it is called electron impact ionization,

$$
e^{-}+A \longrightarrow 2 e^{-}+A^{+},
$$

when the excitation is due to photons it is photoionization

$$
h \nu+A \longrightarrow e^{-}+A^{+},
$$

and when it is due to energized ions/neutral particles it is Penning ionization

$$
A+M^{*} \longrightarrow e^{-}+A^{+}+M .
$$

In a DBD only the electron population is at high energies; therefore the majority of ionization occurs through electron impact ionization. However, high energy electrons commonly excite photon emission and excite vibrational and electronic states which then cause both photoionization and Penning ionization. Because of the minimal emissions and the large ionization energies of $\mathrm{CO}_{2}$, the effects of both photoionization and Penning ionization are considered minimal in a DBD. It should be noted that in other plasma chemical systems the compounded effects of these different ionization paths could have subtle effects on the plasma dynamics and chemistry, for instance by removing energy from vibrational states and 
causing diffusion of energy away from the reaction area.

Once formed, $\mathrm{CO}_{2}^{+}$may react a number of ways, including any method of $\mathrm{CO}_{2}$ dissociation already discussed. The most common reactions are dissociative recombination and radiative recombination-see Table 2.2. These recombination processes occur when an electron recombines with an ionized $\mathrm{CO}_{2}$ molecule. The change in potential as the electron recombines excites the molecule, which then decays either through dissociation, excitation, photon emission, or a combination of the three $[14,51,52]$. The recombination reactions are

Table 2.2: $\mathrm{CO}_{2}$ Recombination reactions

\begin{tabular}{llll} 
Reaction & & $\Delta \mathrm{H}(\mathrm{eV})$ \\
\hline Diss Recomb, rxn A & $\mathrm{CO}_{2}^{+}+e^{-} \rightarrow \mathrm{CO}_{2}^{*}$ & $\rightarrow C O+O$ & -8.3 \\
Diss Recomb, rxn B & $\rightarrow C+\mathrm{O}_{2}$ & -2.3 \\
Rad Recomb, rxn C & $\rightarrow \mathrm{CO}_{2}+h \nu$ & -13.76
\end{tabular}

exothermic and have no activation energy (no initial bonds need to be broken for activation). Measurements using the heavy-ion storage ring ASTRID and CRYRING (CRYRING data is in parentheses) have shown that the branching ratios for the three reactions heavily favor dissociation with rxn A occuring $87 \pm 4 \%(100 \pm 6 \%)$ of the time, rxn B $=9 \pm 3 \%(0 \pm 4 \%)$ and rxn $\mathrm{C}=4 \pm 3 \%(0 \pm 2 \%)[53,54]$. However, if these reactions are to efficiently dissociate $\mathrm{CO}_{2}$, the excess energy after dissociation needs to be spent on further dissociative processes. According to work by Tsuji, et al., 91-98\% of excess energy from rxn A is deposited in the CO vibrational states and according to Wilson et al., the vibrational-vibrational coupling between $\mathrm{CO}_{2}$ and $\mathrm{CO}$ at low temperatures is fast $[55,56]$. The combination of these two processes yields a reaction pathway that should be relatively efficient and can be a major factor for dissociation when a large enough population of $\mathrm{CO}_{2}^{+}$ions are present. 


\section{Argon and Charge Exchange}

Argon was added to the $\mathrm{CO}_{2}$ to reduce the bias voltages needed for plasma breakdown. Argon, despite having a higher ionization energy than $\mathrm{CO}_{2}\left(\mathrm{E}_{i o n, A r} \sim 15.8 \mathrm{eV}\right.$ compared to $\mathrm{E}_{\text {ion, } \mathrm{CO}_{2}} \sim 13.8 \mathrm{eV}$ ), reduces the bias voltage of the plasma due to its small recombination and negative ion formation rate [42]. The decreased recombination rates allow for large populations of $\mathrm{Ar}^{+}$to form in $\mathrm{Ar}-\mathrm{CO}_{2}$ plasmas. However, due to charge-exchange, $\mathrm{Ar}^{+}$is short lived. [57]

$$
\mathrm{Ar}^{+}+\mathrm{CO}_{2} \longrightarrow \mathrm{Ar}+\mathrm{CO}_{2}^{+} \Delta \mathrm{H}=-2 \mathrm{eV}
$$

Ar- $\mathrm{CO}_{2}$ charge exchange is exothermic and only involves an exchange of a single electron, thus the reaction requires no activation energy and occurs quickly [14]. Since the energies involved in this transfer of charge are relatively small (a few eV), the excess energy is often coupled into the vibrational and rotational states of a molecule. Therefore, the addition

of argon effectively increases the $\mathrm{CO}_{2}^{+}$population and excites the vibrational and rotational states of $\mathrm{CO}_{2}$, which increase the $\mathrm{CO}_{2}$ recombination rates and dissociation rates. The effects of different gases with differing charge exchange rates on $\mathrm{CO}_{2}$ dissociation were shown by Suib et al. and Zheng et al. using $\mathrm{Ar}, \mathrm{He}, \mathrm{N}_{2}$, and $\mathrm{O}_{2}[58,59]$. Suib et al. even mentions that the increase in dissociation correlates to the charge exchange rate, but does not go into detail.

\subsection{Plasma Systems}

Systems optimized for $\mathrm{CO}_{2}$ dissociation must focus on either exciting $\mathrm{CO}_{2}$ 's vibrational states or electronic states while keeping the system at low temperatures and recouping as 
much energy as possible. Lasers are inefficient at excitation and thermal systems are too energy intensive, therefore a good alternative is dissociation in a plasma. Since energy efficiency and high reaction rates are needed for a commercial $\mathrm{CO}_{2}$ dissociation, a low pressure plasma is impractical. Therefore, a low temperature, atmospheric plasma is needed for $\mathrm{CO}_{2}$ dissociation.

\subsubsection{Atmospheric Pressure Plasmas}

A plasma is an electrically neutral medium of ions, electrons and neutral particles. Due to the large population of charged particles, a plasma is strongly affected by both electric and magnetic fields and exhibits collective effects. The plasma criteria are quantified by [60]:

1. $n \lambda_{D}^{3}>>1$, the Debye radius $\left(\lambda_{D}\right.$, the radius at which a particle's electric field is completely shielded by nearby charged particles) of charged particles in the system must encompass many other charged particles. ( $n$ is the charged particle density)

2. $\lambda_{D}<<L$, the Debye radius must be smaller than the system size $(L)$

3. $\omega_{e, i}>>\omega_{\text {coll }, e, i}$, the plasma frequency $\left(\omega_{e, i}\right), e=$ electron, $i=$ ion) must be much larger than the the collision frequency of ions and electrons with neutrals $\left(\omega_{\text {coll }, e, i}\right)$

The above criteria are easily satisfied by low pressure plasmas. In a low pressure plasma, the distance between particles is large enough that once ionization occurs, ions and electrons freely experience the effects of external electric and magnetic fields and the internal electric and magnetic fields created by other nearby charged particles. A particle's interaction with both the external and internal electric and magnetic fields is what creates the unique, collective behavior of a plasma. 
An atmospheric pressure plasma (atmospheric plasma) does not easily satisfy the plasma criteria. In contrast to low pressure plasmas, atmospheric plasmas have a large neutral collision rate, which is often close to the plasma frequency. The higher collision rates have two major effects on the plasma. The first is that thermalization and recombination occur at a faster rate, often leading to a shorter plasma lifetime. Therefore, if an atmospheric plasma does not have an active source of ionization (usually a large electric field), it will die out quickly. Second, due to frequent collisions, charged particles have little time to follow electric and magnetic fields. Unlike low pressure plasmas, confinement using electric and magnetic fields in atmospheric plasmas is ineffective and is instead accomplished through manipulation of the system geometry and gas flow.

\subsubsection{Creating Atmospheric Plasmas}

There are two main methods for creating the electric fields necessary for atmospheric plasmas: high frequency electromagnetic fields and large electric fields. The most common way to create a discharge using electromagnetic fields at atmospheric pressure is through radio frequency $(\mathrm{RF})$ or microwave power. The RF and microwave systems use either an antenna or magnetron, respectively, to focus anywhere from several to thousands of watts of radiation into a cavity filled with a target gas. Creation of an atmospheric plasma using large electric fields generally requires a non-conducting fluid (gas or liquid) in-between two electrodes. The electrodes are biased to a relatively high potential, such that there is a large electric field between them. The electric fields for both methods accelerate any spontaneously ionized particles in the cavity (spontaneous ionization can happen due to thermal breakdown or ionizing radiation) and more often than not this acceleration is dominated by electrons. In the electromagnetic case, the oscillations are too fast for the ions to respond. In the large 
electric field case, the electrons quickly shield the ions from the electric field. [13]. If the accelerated particles gain enough energy to ionize other particles, before colliding and/or recombining with other particles, an ionization cascade occurs. The cascade both forms the plasma and heats the plasma until a negative feedback process, such as increased collision cross-sections due to higher particle temperatures, limits the density and temperature. Even though the electrons dominate the motion in these plasmas, due to collisions the ions in many of these plasmas can have energies close to that of the electrons.

\subsubsection{LTE vs. Non-LTE Plasmas}

Depending on the plasma properties, atmospheric plasmas will either be in Local Thermodynamic Equilibrium (LTE) or non-LTE. A plasma in LTE is defined as a plasma having similar localized ion and electron temperatures. It is often much more difficult to heat ions in comparison to electrons, therefore achieving thermodynamic equilibrium is often dependent on ion heating. In a low pressure plasma, ion heating often occurs due to the presence of an external field-either electromagnetic fields or strong electric fields. Electron-ion collisional heating is often minimal in low pressure plasmas, due to the poor energy transfer efficiencies of electron-ion collisions and the small collision cross-sections. In an atmospheric plasma the

roles are reversed; the large collision cross-sections lead to very little external ion heating (the fields don't have time to accelerate the ions before they collide or recombine). Therefore electron-ion collisions are almost exclusively responsible for ion heating. Since the energy transfer efficiency is low for electron-ion collisions, an LTE plasma at atmospheric pressure consists of a large population of high energy electrons, which, through a large number of collisions, heat up the ions to similar temperatures. If the electrons in an atmospheric plasma have enough energy to ionize the system and form a plasma, but the number of electron 
collisions with ions is limited such that ions can not be efficiently energized, the plasma will not be in thermal dynamic equilibrium, i.e. non-LTE. In most cases, non-LTE atmospheric plasmas usually have a large electron temperature and smaller ion and neutral temperatures: an ideal situation for $\mathrm{CO}_{2}$ dissociation through electron excitation.

\subsubsection{Atmospheric Plasma Systems}

A list of the most common atmospheric pressure plasma systems and their parameters is shown in Table 2.3. Of those listed, only the DBD, the atmospheric pressure glow discharge (APGD), the atmospheric pressure plasma jet (APPJ), the corona discharge, and the nonthermal RF discharge are low temperature and excite the electronic states of the plasma species. However, only the DBD, the APGD, and the corona discharge achieve the energies needed for dissociation. Each are viable candidates for a $\mathrm{CO}_{2}$ dissociation reactor based on electron excitation. At standard temperature and pressure (STP), the neutral gas density is $2.4 \times 10^{19} \mathrm{~cm}^{-3}$. Therefore, a comparable plasma density is required for chemical reactions to occur at a reasonable rate. Due to its much higher plasma densities and the ease of scaling up DBDs for commercial use, the DBD was selected as the best choice for a commercially viable system. The choice of a DBD makes it possible to also investigate APGDs, but due to the general instability of the APGD plasma and the limited range of gas mixtures that are able to

form an APGD (none of which contain $\mathrm{CO}_{2}$ ), APGDs were not investigated in this work [61]. Note, recent work by Spencer et al. has shown that atmospheric pressure, non-thermal microwave plasmas have comparable efficiencies to DBDs at $\mathrm{CO}_{2}$ dissociation, especially at low forward powers. However, the system created a high temperature plasma which required significant cooling and like most systems that require resonant cavities, like microwave and RF systems, are difficult to scale up due to the designs resonance requirements. 


\begin{tabular}{|llllll|}
\hline Heating system & $T_{e}(\mathrm{eV})$ & $T_{g}(\mathrm{eV})$ & $n_{e}\left(\mathrm{~cm}^{-3}\right)$ & $\mathrm{T} / \mathrm{NT}$ & source \\
\hline DBD (streamers) & $1-10$ & room temp & $10^{14}-10^{15}$ & $\mathrm{NT}-\mathrm{e}^{-}$excit & {$[61]$} \\
DBD (APGD) & & room temp & $10^{11}-10^{12}$ & $\mathrm{NT}-\mathrm{e}^{-}$excit & {$[62]$} \\
Corona Discharge & $1-10$ & room temp & $10^{9}-10^{14}$ & $\mathrm{NT}-\mathrm{e}^{-}$excit & {$[13]$} \\
APPJ & $1-2$ & room temp & $10^{11}-10^{12}$ & $\mathrm{NT}-\mathrm{e}^{-}$excit & {$[63]$} \\
RF (non-LTE), $\leq 1 \mathrm{~atm})$ & $0.185-2$ & $<0.08$ & $10^{11}-10^{12}$ & $\mathrm{NT}-\mathrm{e}^{-}$excit & {$[13]$} \\
RF (LTE) & $0.6-1.1$ & $0.6-1.1$ & $10^{15}-10^{20}$ & $\mathrm{~T}$ & {$[13]$} \\
Microwave (LTE) & $0.7-9$ & $0.1-1$ & $10^{11}-10^{15}$ & $\mathrm{~T}$ & {$[13,23]$} \\
Arc plasma & $0.8-1.4$ & $0.8-1.4$ & $10^{15}-10^{20}$ & $\mathrm{~T}$ & {$[13]$} \\
Heat + Catalyst & $0.3-0.5$ & $0.3-0.5$ & $\mathrm{NA}$ & $\mathrm{T}$ & {$[27]$} \\
\hline
\end{tabular}

Table 2.3: Plasma discharge parameters for different atmospheric plasmas. $T_{e}$ is the electron temperature, $T_{g}$ is the gas temperature and $n_{e}$ is the electron density. T means thermal excitation, NT means non-thermal excitation and NA means "not applicable."

\subsubsection{The Dielectric Barrier Discharge (DBD)}

To better understand the physics of a DBD, the electrical arc is reviewed. An electrical arc is an LTE plasma created using two electrodes and a non-conducting fluid in between the electrodes. Following the Raether, Meek and Loeb streamer theory (Paschen's Law corrected for high pressure discharges), the discharge forms when the electric field between two electrodes is large enough to start an ionization cascade within the non-conducting fluid [64]. When the cascade occurs, the gas breaks down and the electrons and ions in the fluid are quickly accelerated to the cathode and anode, respectively. The breakdown often occurs within a very thin beam and forms along the shortest path between electrodes. The reason a thin beam forms is explainable through the example of two parallel plate electrodes with an air gap. Each path between electrodes in a parallel plate is the same length to start, so once breakdown conditions are met the discharge occurs uniformly across the electrode surfaces, with some areas being slightly higher and lower density due to various effects like the thermal distribution, local collision rates, and areas of pre-ionization. The 
higher plasma density regions have a lower resistance to ion and electron conduction due to fewer collisions with slow neutrals and charged particles, thus more electrons and ions flow through those regions. This in turn increases the plasma density in the higher density region making conduction even easier, causing other higher resistance paths to die out until there is a single, high density plasma in the form of a thin and/or branching filament from the anode to the cathode. The collapse of the discharge paths into a single filament (assuming a short discharge gap $<1 \mathrm{~cm}$ ) happens within several nanoseconds of applying a voltage that exceeds the breakdown threshold. In the case of electrical arcs, the cathode and anode continuously supply and remove electrons from the system making a continuous plasma that is connected to both electrodes, only needing a DC voltage and a large current to keep it running (However, AC sources also work). Despite the very fast particle quenching rate due to the high number of collisions with the surrounding cold particles, the thin plasma channel quickly thermalizes. Thermalization occurs because electrons have a smaller collision crosssection than ions and therefore quickly reach high energies in the electric field. Since the electrodes continuously supply electrons, the ions are constantly bombarded, and therefore heated, by the fast electrons. The bombardment quickly increases the average ion and neutral energies in the arc and, in turn, decreases the collision frequency of the ions, thus allowing the electric field more time to accelerate the ions. These effects combine such that the ions usually have a temperature within a factor of 3 or 4 of the electrons, making an electrical arc a LTE plasma.

The DBD was first developed in 1857 by Ernst Werner Von Siemens for use in ozone production and was originally called the "silent discharge" [65]. The DBD is a high pressure, non-LTE, transient, low temperature plasma discharge. The DBD apparatus is similar to an arc with two electrodes and an air gap, but a DBD has the addition of a high breakdown 
voltage material placed in between the electrodes to impede arcing, often a dielectric. In practice, one electrode is grounded and the other is attached to a high frequency $(60 \mathrm{~Hz}-$ $500 \mathrm{kHz})$, high voltage $(0-500 \mathrm{kV})$ power source. The electrode and dielectric geometries have a variety of possible configurations, as seen in Figure 2.3. When voltage is applied to the electrodes, the dielectric charges and a strong electric field quickly develops within the air gap. Once the electric field is strong enough that ions and/or electrons are accelerated to ionization energies, before losing energy to collisions, the ionization cascade occurs. Different from an arc, when the cascading particles, either ions or electrons, reach the dielectric they accumulate on the surface and are not conducted out of the system. The charge buildup cancels the charge on the electrode and lowers the effective electric field within the air gap; preventing an electrical arc and further acceleration of the charged particles. Shielding continues after the initial cascade due to charge buildup on the surface as well as a sheathe region in front of the cathode and/or dielectric surface (supplies new charge to the surface as charged particles are neutralized). The shielding process occurs over tens to hundreds of nanoseconds and, once enough charge is collected on the surface, the DBD discharge begins to dissipate [66-71]. For the discharge to continue, the electric field is reversed and the cascade, often fueled by the previous wave of ionization, occurs in the opposite direction.

Due to the accumulation of charge on the dielectric surface, the ionization cascade in DBDs transport only a small amount of charge before the local electric field is shielded and particles no longer gain enough energy to continue the cascade. Since this shielding is localized, a DBD often does not consist of a single filament, but instead forms a streamer discharge which consists of multiple filaments distributed across the discharge area. Since each filament requires a similar electric field to form, each filament is practically identical (assuming no anisotropies in the DBD design, such as pointed electrodes). In the case of 

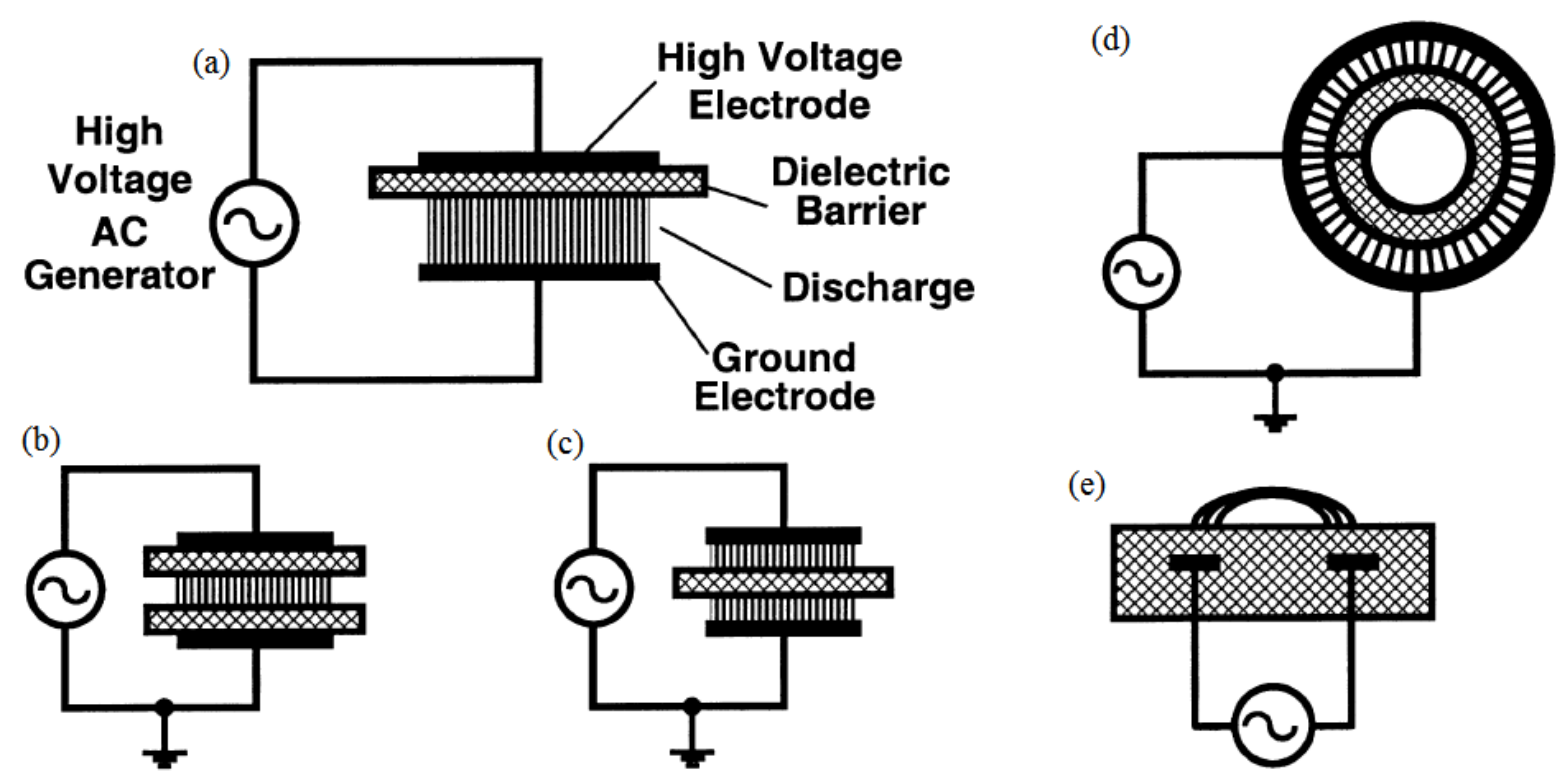

Figure 2.3: Several DBD configurations [65]. (a), (b) and (c) are different configurations for planar DBDs, (d) is an end on view of a cylindrical DBD and (e) is a surface DBD or more commonly known as a plasma actuator.

a DBD, these filaments are called streamers: short-lived, non-LTE, filament-like plasmas created by a large electric field.

Depending on the sign of the voltage, the streamer either cascades like an arc or it can have a cathode directed streamer. The arc-like cascade is depicted in the four left most diagrams of Figure 2.4 and generally starts at or near the cathode and cascades to the anode with a large wavefront of electrons. The electron wavefront quickly ionizes particles as they move through the gap, which spreads the discharge out and builds up the electron density. In the wake of the wavefront, a population of ions and enough electrons for quasi-neutrality are left behind to shield the electric field from the rest of the plasma, therefore only charges in the wave-front are affected by the external electric field. When the electrons reach the surface, they build up there while the plasma wake continues to keep quasi-equilibrium. With the external electric field completely shielded, the plasma begins to cool. As DBDs 


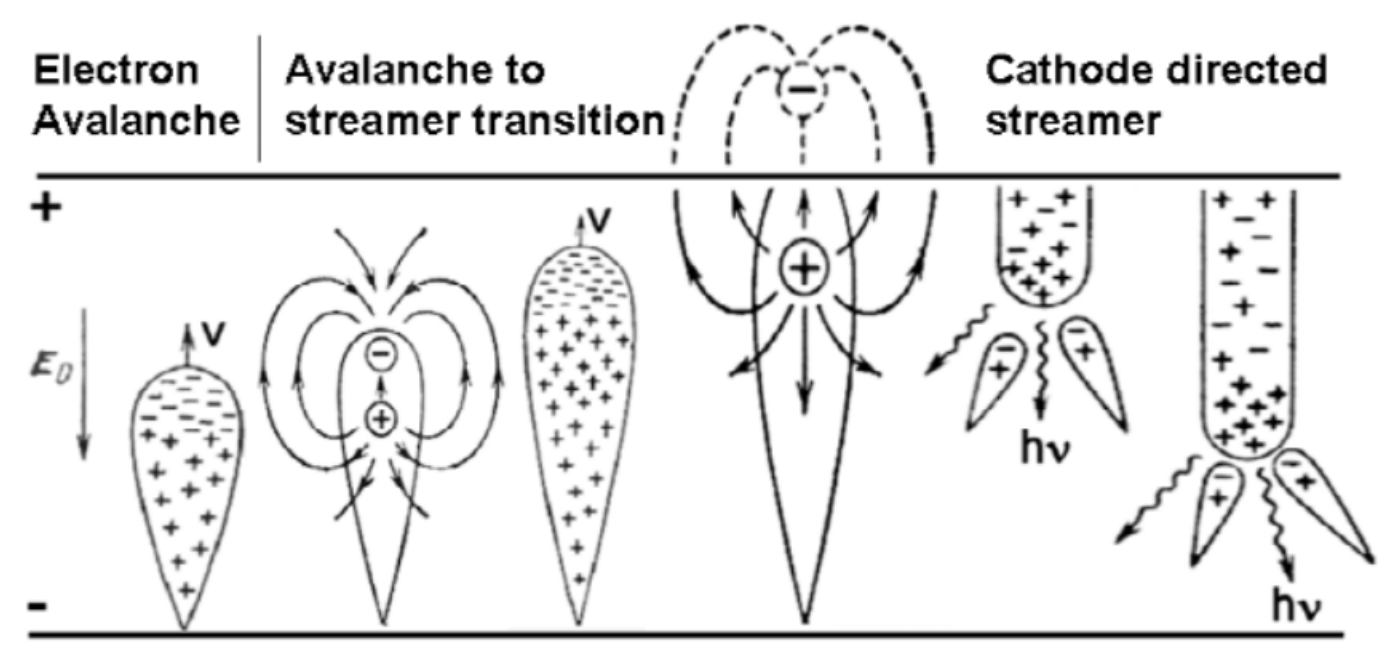

Figure 2.4: Illustration of Streamer Formation. Picture taken from Ref. [72]

generally have a high voltage (HV) electrode and a ground electrode, this type of streamer occurs when the HV electrode is charged positively. When the HV electrode is charged negatively, the cathode directed streamer occurs. The cathode directed streamer begins with electrons being pushed away from the anode (HV electrode) and causing a cascade. Once the cascade reaches a density where the plasma effectively shields external electric fields, the wavefront no longer sees an electric field and ceases to cascade (since the electron wavefront only sees the ground electrode). However, the conductive plasma in the wake of the electron cascade effectively shortens the distance between electrodes, thus compressing the electric field between it and the electrode $[67,73]$. Electric field compression increases the acceleration of particles in the cascade and radiation from the previous plasma wave creates electron-ion pairs ready to form the next cascade, as seen in the two right most diagrams in Figure 2.4. The new cascades then connect up with the previous cascades and further compress the electric field. This process of compressing and cascading continues until the plasma reaches both electrodes, thus forming a cathode directed streamer. Note, electric field compression also occurs during arc-like cascades and similarly accelerates particles at 
the head of the cascade. The effects of these two cascade types on the chemistry of $\mathrm{CO}_{2}$ dissociation is expected to be minimal, therefore it was not considered during this analysis.

After the initial cascade is finished, the streamer generally looks like an hourglass, see Figure 2.5, which consists of three regions: two surface discharge regions and the microdischarge channel. The surface discharge is the region in which the plasma is in contact with

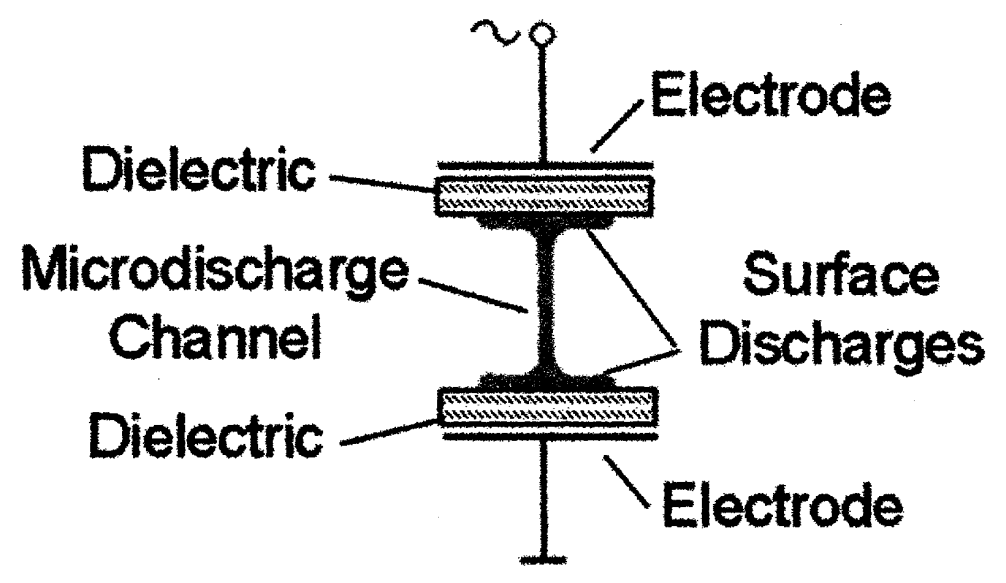

Figure 2.5: A single streamer in a DBD with dielectric covering both electrodes. Picture taken from Ref. [73]

either a dielectric or electrode surface. The diameter of the surface discharge is generally between $1 \mathrm{~cm}$ to $1 \mathrm{~mm}$, which is significantly wider than the micro-discharge channel. This change in plasma widths is due to the opposing electric field of the charges on the dielectric surface which deflect impinging particles. The surface discharge diameter is reduced on electrode surfaces due to electrons being conducted out of the system. The spreading that does occur is due to secondary electrons and photons created by particles impinging on the conductive surface (which then cause photo-ionization), with a small amount attributed to ion build up and diffusion across the surface. The micro-discharge channel is the plasma outside of the surface discharge regions and is most similar to the initial cascade of an arc plasma, consisting of a very thin dense column of plasma connecting the two surface 
discharges. The typical plasma characteristics of the streamer are listed in Table 2.4 [61].

Table 2.4: General characteristics of an atmospheric pressure streamer. Density and temperature values listed are for the center of the discharge and the magnitudes decrease with distance from the center of the streamer [61]

\begin{tabular}{|ll|ll|}
\hline$n_{e}$ & $10^{14}-10^{15} \mathrm{~cm}^{-} 3$ & Filament radius & $\sim 10^{-3}-10^{-4} \mathrm{~m}$ \\
$T_{e}$ & $1-10 \mathrm{eV}$ & Streamer Duration & $1-10 \mathrm{nsec}$ \\
$T_{g}$ & $<400 \mathrm{~K}$ & Peak current & $0.1-1 \mathrm{~A}$ \\
\hline
\end{tabular}

In a parallel plate DBD, the streamers will often move across the dielectric/electrode surfaces with an erratic behavior while dissipating and reforming seemingly randomly, even when there is no gas flow. The streamer motion is due to the relatively long lifetime of charged particles in an atmospheric pressure system. If the lifetime of charged particles is longer than the time it takes for the power supply to switch signs, the remaining charged particles in the micro-discharge channel fuel the re-ignition of the same streamer. Repeated re-ignition makes each streamer look and often act as if it is a continuous discharge-this is called the memory effect [61]. The random motion is due to each streamer being an electric dipole oriented in the same direction as other streamers, the ions closer to the anode and the electrons closer to the cathode. The dipole-dipole interaction causes streamers to repel one another. If the repulsion pushes a streamer into an area with a weaker electric field, the streamer may not reignite when the electric field changes sign. The dissipation of a streamer means the electrodes are less shielded, which leads to an increase in the effective electric field strengths and creates the opportunity for new streamers to form in areas of stronger electric fields. The forming and dissipation of streamers is strongest when the electric field oscillates quickly (depending on the gas, system flow rate, etc) and at large Townsend (Td, $\left.10^{-17} \mathrm{~V} / \mathrm{cm}\right)$ values $(>100 \mathrm{Td})$. When the electric field oscillation frequency is small, such that charged particles have a chance to recombine or drift out of the reaction area before the 
electric field reverses, reignition is less frequent and the streamers will form and dissipate in a more random fashion. Logically, this is also affected by the number of streamers, which is dependent on the electric field. Therefore, one way to reduce the randomness is to reduce the electric field and in turn the number of streamers that are interacting with one another. When the number of streamers needed to shield a surface becomes relatively small, the streamers can reach an equilibrium where each or a portion of the streamers are stationary. In highly uniform situations, DBD discharges have been shown to form a grid/pattern [74]. Stationary discharges can also be created using different electrode geometries. For example, the charge on an electrode will accumulate at points and sharp edges, creating larger electric fields at those positions. Since those positions will often have electric fields much greater than the surrounding areas, the streamers at these location will resist movement and become stationary. Pointed and/or sharp electrodes have the side effect of reducing the electric field in other locations, which in turn lowers the number of streamers that form, thus reducing the plasma volume. However, the charge accumulation at points and edges can be done intentionally in order to create a corona discharge, which uses the enhanced electric fields to break down the surrounding gas and create a different type of filament-like plasma.

\subsubsection{DBD Plasma Dynamics}

The low temperature, non-LTE nature of DBDs results from the dynamics of particle motion in an external electric field. The force and energy equations for a charged particle in a uniform 
electric field are

$$
\begin{gathered}
\vec{v}=\vec{a} t+\vec{v}_{0} \quad \vec{F}=m \vec{a}=q \vec{E} \rightarrow \vec{a}=\frac{q \vec{E}}{m} \\
K E=\frac{1}{2} m v^{2}=\frac{1}{2} m\left(a t+v_{0}\right)^{2}=\frac{q^{2} E^{2}}{2 m} t^{2}+q E v_{0} t+\frac{1}{2} m v_{0}^{2}
\end{gathered}
$$

where, $\vec{v}$ is the velocity, $\vec{v}_{0}$ is the initial velocity, $\vec{F}$ is the force, $m$ is the particle mass, $\vec{a}$ is the acceleration, $q$ is the particle charge, $\vec{E}$ is the electric field, $K E$ is the kinetic energy and $t$ is the particle's time in the electric field. The quantity $\frac{q^{2} E^{2}}{2 m} t^{2}$ is the amount of energy a particle gains from being accelerated in an electric field and the $q E t v_{0}$ term is the energy a particle gains due to moving through the electric field with an initial velocity. These terms have a $1 / m$ and a $\sqrt{1 / m}$ (within the $v_{0}$ term) dependence, respectively. Thus, electrons will gain more than 40 times as much energy from the electric field compared to an ion given similar initial conditions and flight times. As will be shown, the difference in masses, the lowering of the collision cross-section with velocity and the large collision rates at atmospheric pressures lead to electrons gaining significantly more energy in a DBD compared to the ions.

\section{Cross-Section Calculations}

The approximate collision cross-sections of the ions and electrons in an electric field were calculated to determine the amount of energy the electric field deposits into the various particles between particle collisions, and where the accelerated particles deposit their energy during a collision. The collision cross-sections for each plasma interaction were calculated, including electron (e)-ion collisions, e-e collisions, e-neutral, ion-ion, ion-e and ion-neutral (test particle-field particle). Neutral-neutral collision cross-sections were not calculated because they do not change the energy stored in the neutrals. The large angle collision cross-section 
and the small angle collision cross-section were calculated at various electron temperatures and electron/ion densities. The large angle cross-section $\left(\sigma_{L}\right)$ was calculated from

$$
\sigma_{L} \approx \pi b_{\pi / 2}^{2}=\pi\left(\frac{q_{T} q_{F}}{4 \pi \epsilon_{0} \mu v_{0}^{2}}\right)^{2} \quad b_{\pi / 2}=\frac{q_{T} q_{F}}{4 \pi \epsilon_{0} \mu v_{0}^{2}}
$$

where $b_{\pi / 2}$ is the impact parameter for $90^{\circ}$ collisions, $q_{X}$ is charge $(T=$ test particle, $\mathrm{F}=$ field particle), $\epsilon_{0}$ is the permittivity of free space, $\mu$ is the reduced mass of the colliding particles, $v_{0}$ is the initial relative velocity between the test particle and field particle. The small angle collision cross-section $\left(\sigma_{S}\right)$ :

$$
\sigma_{S}=8 \ln \left(\frac{\lambda_{D}}{b_{\pi / 2}}\right) \sigma_{L}=\frac{1}{2 \pi}\left(\frac{q_{T} q_{F}}{\epsilon_{0} \mu v_{0}^{2}}\right)^{2} \ln \left(\frac{\lambda_{D}}{b_{\pi / 2}}\right)
$$

where $\lambda_{D}$ is the Debye radius. The sum of these two cross-sections gives the total crosssection for collisions between two charged particles $\left(\sigma_{t o t}\right)$.

$$
\sigma_{\text {tot }}=\sigma_{S}+\sigma_{L}=\sigma_{\text {Largeangle }}\left(1+8 \ln \left(\frac{\lambda_{D}}{b_{\pi / 2}}\right)\right)
$$

For the collision cross-section between the charged particles and neutrals, trajectories can be considered ballistic and $\sigma_{\text {neutral }} \sim 3 * 10^{-16} \mathrm{~cm}^{2}$, based on an average particle radius of $\sim 10^{-8} \mathrm{~cm}$. The collision cross-section derivations and values reported here come from sections 1.8-1.10 of Bellan [75]. The collision frequency $(\nu)$ was calculated from

$$
\nu=N\langle\sigma v\rangle \quad \text { where } \quad\langle\sigma v\rangle=\frac{\int v \sigma(v) f(v) d v}{\int f(v) d v}, \quad f(v)=e^{-\frac{\left(m v^{2}\right)^{2}}{\left(2 k T_{e}\right)^{2}}}
$$


where $N$ is the density of the target particles, $\langle\sigma v\rangle$ is the collision rate averaged over the distribution of velocities and $f(v)$ is a Druyvesteyn velocity distribution function. The collision frequency is the rate at which a particle collides with other particles, and was broken down by species to identify which collisions dominate the reaction kinetics of the different plasma components.

Equation 2.15 was solved to find the average energy a charged particle gains from the electric field before colliding with a particle and losing its energy. The inverse of the collision frequency was used for $t$, because it gives the average time a particle travels before colliding with a particle and undergoing a large angle scattering event. The other variables used in the calculation are given in Table 2.5.

Table 2.5: Variables used for calculating the average energy a particle gains from the electric field within a DBD

\begin{tabular}{|ll|ll|}
\hline Charge, $q$ & $1.6022 \times 10^{-19} \mathrm{C}$ & Electric field, $E$ & $20 \mathrm{kV} / \mathrm{cm}$ \\
Initial ion velocity, $v_{0, i}$ & $6.92 \times 10^{4} \mathrm{~cm} / \mathrm{s}(0.033 \mathrm{eV})$ & e- mass, $m_{e}$ & $9.1094 \times 10^{-28} \mathrm{~g}$ \\
Initial e- velocity, $v_{0, e}$ & $3.75 \times 10^{7} \mathrm{~cm} / \mathrm{s}(0.5 \mathrm{eV})$ & Ion/neutral mass, $m_{\text {ion }}$ & $1.6726 \times 10^{-24} \mathrm{~g}$ \\
e- $/$ ion density, $n_{e} / n_{i}$ & $10^{13}-10^{16} \mathrm{~cm}^{-3}$ & e- Temperature, $\mathrm{T}_{e}$ & $0-10 \mathrm{eV}$ \\
\hline
\end{tabular}

The density and temperature ranges chosen for the calculations included values above and below the normally reported densities and temperatures of DBD systems (see Table 2.4). The ion mass was set to the minimum value (mass of a proton) to show the effects of the electric field on the most agile of ions in the system. Due to the low energies measured in DBD plasmas and the unreasonably large electron and ion energies found for initial energies above $1 \mathrm{eV}$, a moderate initial energy of $0.5 \mathrm{eV}$ for electrons and $0.033 \mathrm{eV}$ for ions was chosen. 


\section{Collision Cross-Section Results and Analysis}

Figure 2.6 shows the results of the collision cross-section analysis for each of the different collision types for varying electron densities and temperatures. The collision frequency was calculated using the results of the collision cross-section calculations, which were then used to find the average energy a particle gains before colliding with another particle. The collision frequency is shown in Figure 2.7 and the average energy gain in Figure 2.8. Note, this analysis is only applicable to the discharge cascade wavefront, because the wavefront shields the bulk of the plasma from the external electric fields, thus leaving a cool plasma in its wake. 

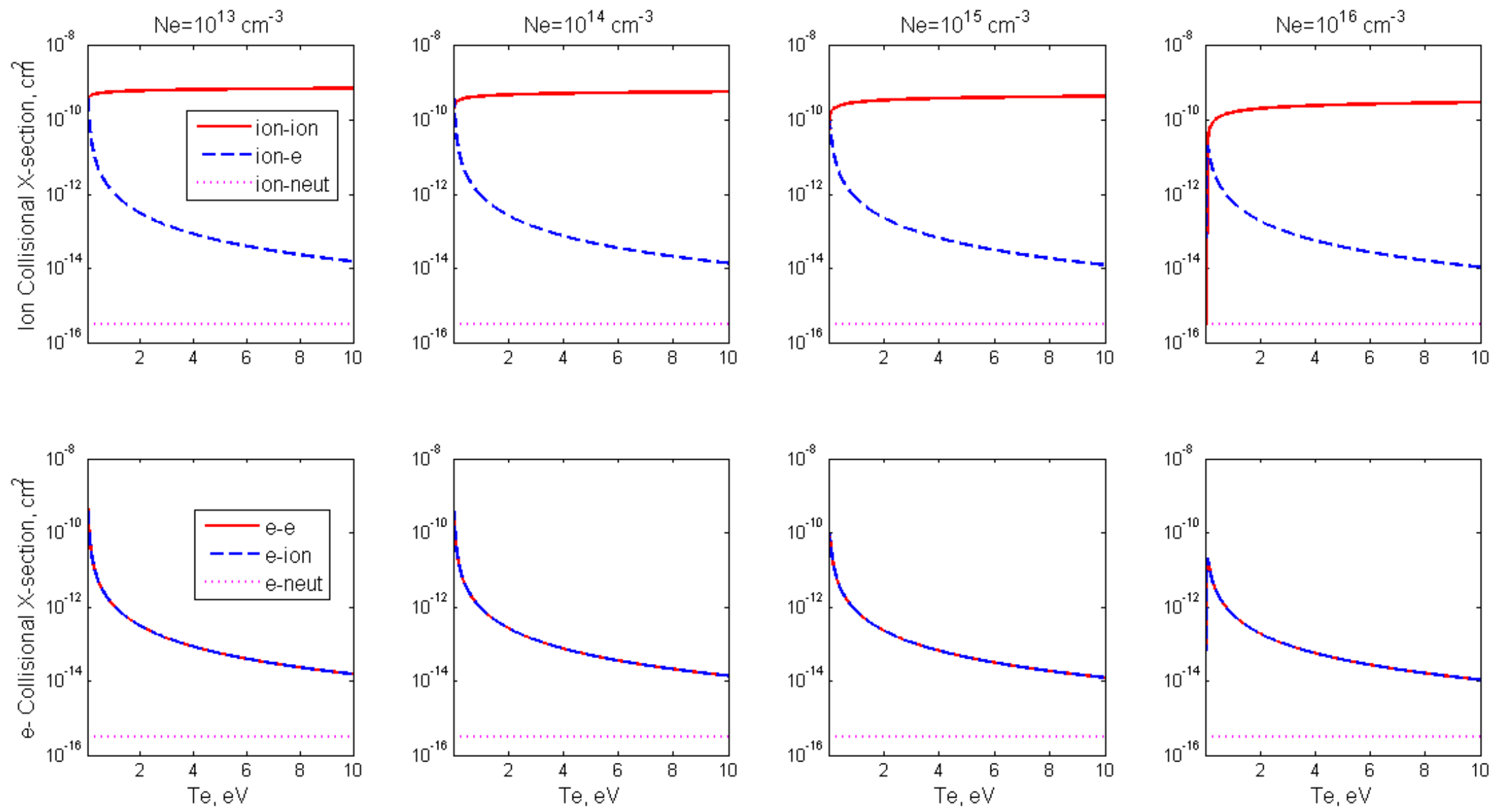

Figure 2.6: The calculated cross-section of ions (top graphs) and electrons(bottom graphs) with other ions, electrons and neutrals versus the electron temperature in an atmospheric DBD plasma at different electron and ion densities. Calculated using Equation 2.18 and the values shown in Table 2.5. The e-e and e-ion cross-sections overlap one another. 

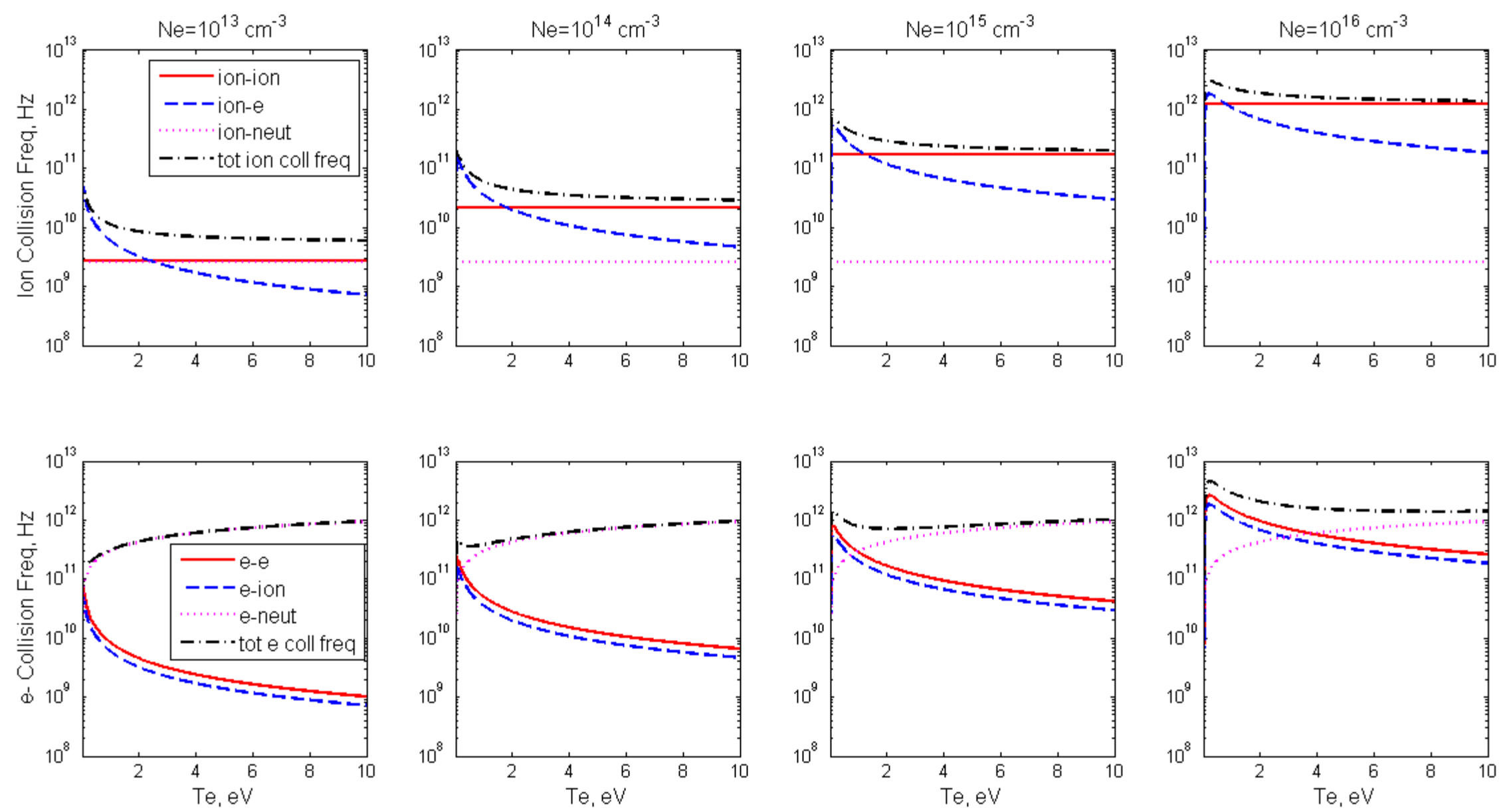

Figure 2.7: The calculated collision frequency of ions (top graphs) and electrons (bottom graphs) with other ions, electrons and neutrals versus the electron temperature in an atmospheric DBD plasma at different electron and ion densities. These values were calculated from the results of Figure 2.6, using Equation 2.19 and the variables given in Table 2.5 . 

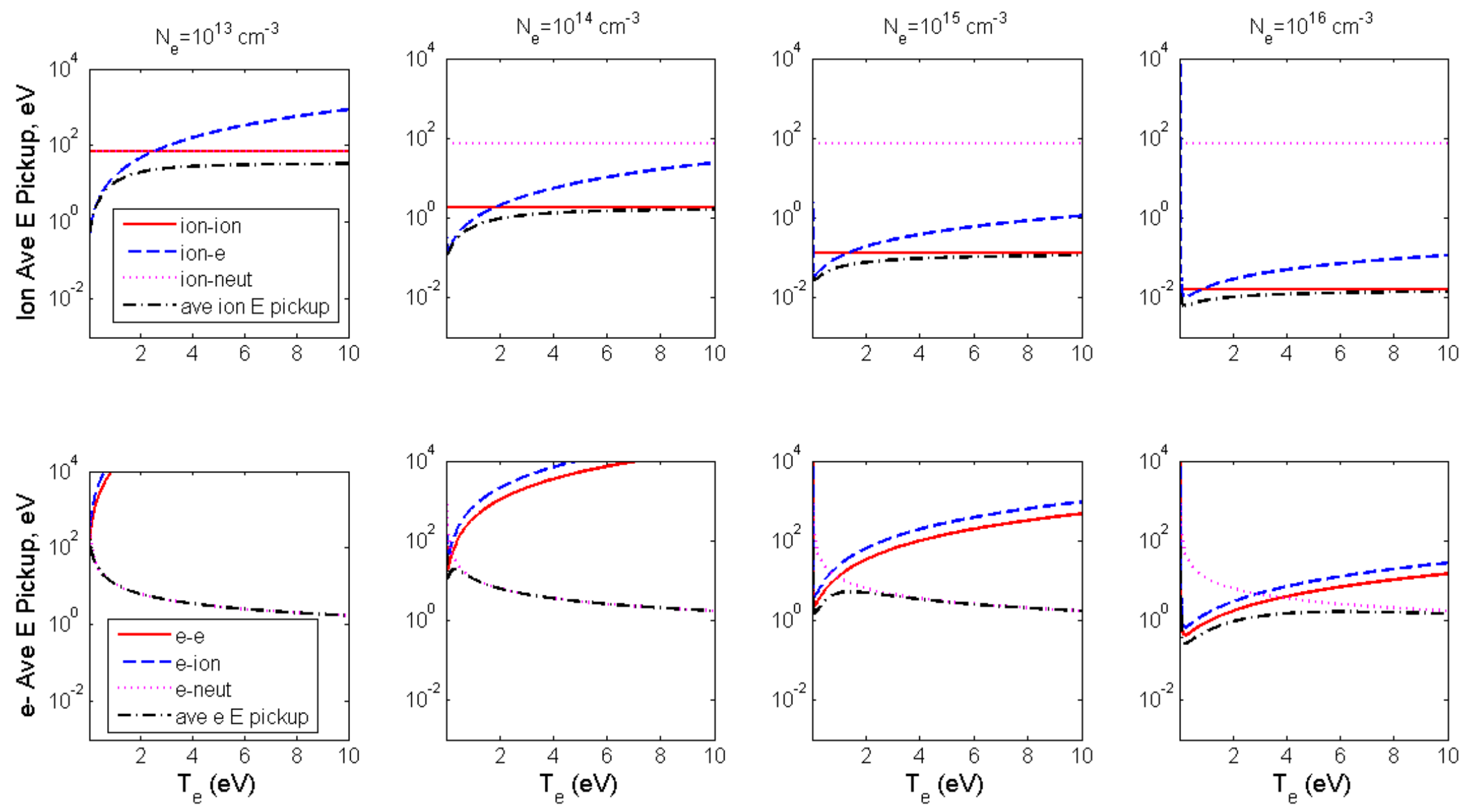

Figure 2.8: The average energy ions (top graphs) and electrons (bottom graphs) gain from the electric field before colliding with other ions, electrons and neutrals versus the electron temperature in an atmospheric DBD plasma at different electron and ion densities. Calculated from the results of Equation 2.7 and values given in Table 2.5. The curves with the lowest energy indicate the particle that most often collides with the target particle and dominates the target particle's energy. 
The first things to notice from Figures 2.7 and Figures 2.8 are the electron and ion collision frequencies for densities between $10^{13}-10^{14} \mathrm{~cm}^{-3}$; these are the typical densities found during plasma formation. Figure 2.7 shows that the electron collision frequency is smaller at lower electron temperatures, which makes the electron energy gain high for low energy electrons. The large energies acquired by these low energy electrons quickly initiate the electron avalanches and increase the density of the plasma. What is interesting are the ion energy and collision frequencies at low densities. When the electron temperature is low, the ions gain little energy from the electric field due to the ion's large collision rate with low energy electrons, which results in recombination. However, when the electron temperature is above $\sim 0.5 \mathrm{eV}$, the electron-ion collision rate drops such that ions gain significant energy from the electric field and collide with other ions and neutrals more frequently than electrons. Therefore, at the beginning of the discharge it can be expected that there is a small population of high energy ions [76]. If a plasma system was capable of achieving relatively high electron energies with limited electron densities, significant ion acceleration could be possible in these plasmas.

The electron and ion collision frequencies and energy gains from the electric field at higher plasma densities $\left(10^{15} \mathrm{~cm}^{-3}\right)$ help explain the steady state plasma dynamics generally observed in DBDs. Consistent with DBD theory, the graphs show that electrons mainly collide with neutrals and gain upwards of several $\mathrm{eV}$ from the electric field (this is even greater at higher electric fields), whereas the ions mainly collide with other ions and electrons, and gain little energy $(<0.1 \mathrm{eV})$ from the electric field at all electron temperatures. Therefore, the results give strong evidence for electrons being the main source of ionization in a DBD. DBDs also are often considered to have a limit to both their electron density and temperature. These calculations show that higher densities $\left(10^{16} \mathrm{~cm}^{-3}\right)$ lead to lower electron energies $(<1$ 
$\mathrm{eV}$ ) and higher e - ion collision frequencies, which lead to larger recombination rates and a reduction in electron density. Thus, the density is self-limited. The electron temperature is limited by the peak in the electron energy gain graph for electrons at $N_{e}=10^{15} \mathrm{~cm}^{-3}$ at $T_{e} \sim 1 \mathrm{eV}$. At temperatures above or below this peak, the electron energy gain decreases significantly and limits the achievable electron temperature. Also, increases in electron temperature show a decrease in the collision frequency of both electrons and ions, which further increases the plasma density (which is limited by higher recombination rates). The combination of these two negative feedback mechanisms limits the electron temperature. Note, the electron temperature limit is due to the neutral densities, as can be seen by the domination of the electron collision frequencies at higher electron temperatures. Therefore, low pressure DBDs do not have the same temperature limit as atmospheric pressure DBDs.

DBDs are considered to be low temperature plasmas; this designation refers to the ion and neutral temperatures and often implies that the plasma is not hot enough to cause significant sputtering or damage to surfaces (like melting). For example, low temperature plasmas are often used for biological applications [77]. Figure 2.8 shows that the ion energy gain from the electric field drastically decreases as electron density increases and is extremely low at typical DBD densities. Therefore, the electric field does not impart a large amount of energy into the ions. The energy transfer from electrons to ions is calculable from the equations for energy transfer between electrons and ions in an elastic collision

$$
\begin{gathered}
v_{i o n, f i n}=\frac{2 m_{e}}{m_{i o n}+m_{e}} v_{e, i n i} \\
K E_{i o n}=\frac{1}{2} m_{i o n} v_{i o n}^{2} \approx 2 \frac{m_{e}^{2}}{m_{i o n}} v_{e, i n i}^{2}
\end{gathered}
$$


and accounting for the number of collisions that occur within a plasma discharge time scale.

$$
N_{\text {coll }}=\nu \times t_{\text {disc }}
$$

Using the mass of a $\mathrm{CO}_{2}$ molecule $(44 \mathrm{amu})$, the maximum discharge time $\left(t_{\text {disc }}\right)$ and the collision frequency for a streamer $\left(\nu \sim 10^{13} \mathrm{~Hz}\right.$ and $\left.t_{d i s c} \sim 10 \mathrm{~ns}\right)$, we found that the number of collisions is $\sim 10^{5}$ and an electron imparts $\sim .005 \%$ of its energy to a stationary ion during an elastic collision. If every collision is perfectly elastic and always additive to the ion's energy, this could heat the ions to similar temperatures to the electrons. But, energy is constantly lost through inelastic collisions with atoms and molecules in both the surrounding gas and the boundaries (the dielectric and electrodes). Therefore, the electrons are not a strong source of energy for ions and neutrals. With no strong sources of ion or neutral heating and the electric field only able to accelerate the electrons to high energies, it is not surprising to find that DBDs are both low temperature and non-LTE.

\section{Further Analysis}

The cross-section calculations developed in the previous sections rely on three assumptions: the ions and neutrals are at room temperature $\left(300^{\circ} \mathrm{K}\right)$, the electric field within the air gap is at least $20 \mathrm{kV} / \mathrm{cm}$, and the electron velocity distribution is a Druyvesteyn distribution. The temperature assumption is reasonable because even after prolonged run times the VADER system never exceeded any of the reaction boxes component melting temperatures of $\sim 400^{\circ}$ K. As for the electric field, the TREK power supply (described in Section 3.1.2) has a maximum setting of $\pm 10 \mathrm{kV}$ plus a $1 / 8$ " electrode spacing gives an electric field of $\sim 30 \mathrm{kV} / \mathrm{cm}$ at frequencies up to $2.5 \mathrm{kHz}$. For higher frequencies, the peak voltages were much smaller. 
The dielectric then amplifies the electric field in the air gap (up to a factor of 2 when using equal thicknesses of dielectric and air gap), along with electric field compression effects. However, modeling of these amplified fields (up to $50 \mathrm{kV} / \mathrm{cm}$ ) showed a baseline increase in the average electron energy gain but insignificant effects on the ion energies. Note, the breakdown voltage of the gas determines the electric field during the cascade of a streamer and not the applied voltage, therefore the electric field within each streamer is invariant.

A Druyvesteyn distribution is the energy distribution created when charged particles are accelerated within an electric field. The Druyvestyn distribution has a larger low energy population and a smaller high energy population as compared to a Maxwellian and has been shown to be more appropriate for a DBD than a Maxwellian [78]. Figure 2.9, shows the difference in energy pick up between a Druyvesteyn and a Maxwellian distribution. In the higher electron density cases the difference was as much as 40\%. Carman and Mildren suggest that a bi-Druyvestyn and bi-Maxwellian distributions are more accurate representations of the distribution, because they include second-order terms based on the first atomic energy states of the plasma. However, due to the small first energy states of molecules (for $\mathrm{CO}_{2}$ $<0.1 \mathrm{eV})$ these second order terms are negligible. 

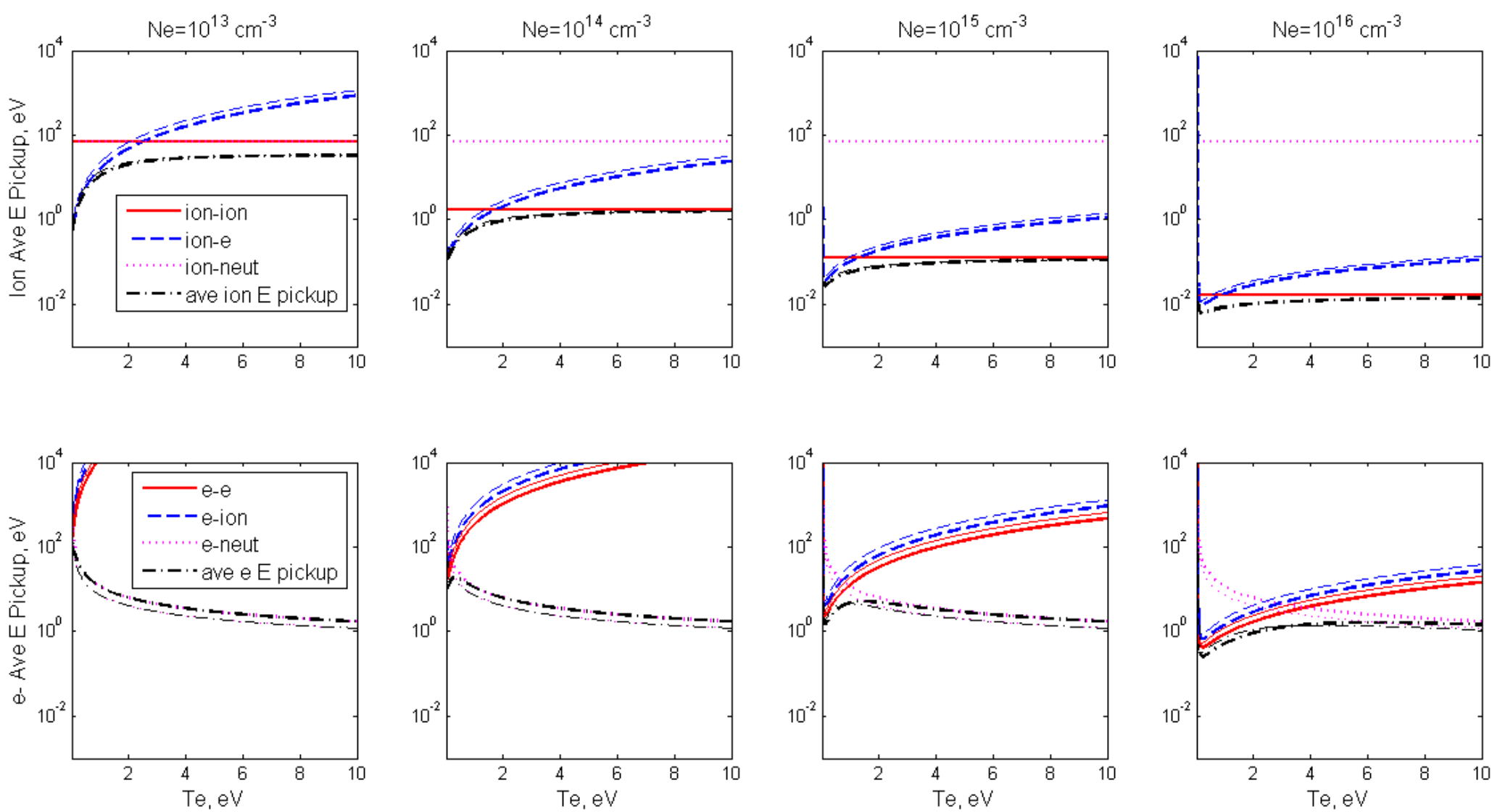

Figure 2.9: Comparison of the Druyvesteyn distribution to the Maxwellian EEDF for the average energy gain for ions (top graphs) and electrons (bottom graphs) in an electric field versus the electron temperature in an atmospheric DBD plasma at different electron and ion densities. The Maxwellian distributions are the thicker lines. The average energy ions (top graphs) and electrons (bottom graphs) gain from the electric field before colliding with other ions, electrons and neutrals versus the electron temperature in an atmospheric DBD plasma at different electron and ion densities. 


\subsection{DBD Plasma Chemistry Model}

Due to the non-steady state features and the timescales involved in a DBD (picosec to msec time-scales), modeling of streamer physics is a difficult task. When complex chemistry is included, full-scale simulation becomes almost impossible. However, until atmospheric plasma diagnostics for DBDs improve, models are the best way to understand DBD plasma chemistry. Therefore, to better understand the $\mathrm{CO}_{2}$ plasma chemistry in VADER we used a simplified model of DBD dynamics based on the results of several models to calculate the reaction rates found within a $\mathrm{CO}_{2}$ streamer. The parameters for the model were based on VADER parameters with a flow rate of 200 standard cubic centimeters per minute (SCCM) with a gas composition of $60 \% \mathrm{CO}_{2} 40 \%$ Ar passed through a $0.3175 \mathrm{~cm}^{2}$ inlet cross-section and a driving frequency of $30 \mathrm{kHz}$. The model was compared to experimental results in which there was $5 \% \mathrm{CO}_{2}$ dissociation using the above parameters, which corresponds to a $\sim 4 \times 10^{-6} \mathrm{~mol} / \mathrm{s}$ conversion rate and $r=\sim 1.25 \times 10^{-6} \mathrm{~mol} / \mathrm{cm}^{3} \mathrm{~s}$. Therefore, reaction rates significantly below $1.25 \times 10^{-6} \mathrm{~mol} / \mathrm{cm}^{3}$ are ignorable. The model assumes that the majority of reactions occur over several overlapping stages of the streamer, the stages are broken down into the cascade phase, the afterglow phase and the ground state phase $[66,73]$. The cascade phase encompasses streamer formation (the cascade) and the shielding of the external electric field. During the cascade phase, electrons are at relatively large energies (1-10 eV), meaning most of the high energy endothermic reactions occur at this time. Once the electric field is shielded, the streamer enters the afterglow phase. At which point the electron temperature and density quickly drop as electrons collide with particles and begin recombining at a high rate. The recombination of electrons with ions and the high energy reactions during the cascade phase lead to the afterglow phase, which is dominated by free radical chemistry 
(discussed in Section 2.1.4). Approximately 1 msec after the initial discharge the population of free radicals becomes small enough that ground state chemistry is dominant. Due to the small neutral temperatures found in a DBD afterglow plasmas, $\mathrm{CO}_{2}$ neutral chemistry is slow and the process favors reverse reactions. Therefore, DBDs for $\mathrm{CO}_{2}$ dissociation are often pulsed at frequencies such that time in the ground state phase is minimized.

\subsubsection{The Cascade Phase}

Models predict that the time for the initial cascade to propagate from one electrode to the other takes between several to hundreds of nanoseconds and is highly dependent on the gas makeup, ionization energies and the applied electric fields [66-71]. Within the first nanoseconds of the cascade, the electron and ion densities reach a maximum of anywhere from $10^{12}-10^{15} \mathrm{~cm}^{-3}$ with average electron temperatures between $1-10 \mathrm{eV}$, at which point the electron and ion formation rates begin to dissipate due to shielding. During these several nanoseconds of high energy and density electrons, electron impact reactions are dominant and endothermic reactions are possible.

The e- $\mathrm{CO}_{2}$ reaction rates were solved for typical cascade temperatures, $T_{e}=1-10 \mathrm{eV}$, using

$$
r\left(\frac{\text { moles }}{c m^{3} s}\right)=\int_{0}^{\infty} f\left(v_{e}\right) \sigma\left(v_{e}\right) v_{e}[A] \mathrm{d} v_{e} \times L
$$

where $r$ is the reaction rate, $v_{e}$ is the electron velocity, $f\left(v_{e}\right)$ is the electron velocity distribution function using a Druyvesteyn distribution, $\sigma\left(v_{e}\right)$ is the electron- $\mathrm{CO}_{2}$ cross-sections, $[A]$ is the target ion or neutral density and $L$ is the scaling factor. A list of the relevant electron- $\mathrm{CO}_{2}$ collision reactions and their corresponding cross-sections is tabulated in Appendix B. A minimal electron density of $n_{e}=10^{12} \mathrm{~cm}^{-3}$ was used to give a lower limit on 
the important reaction rates occurring in the streamer. The Druyvesteyn distribution was considered one-dimensional as electrons are only accelerated in the direction of the electric field and, due to the vast difference in energies and masses, the ions and neutrals were considered stationary in comparison to the electrons. Since the plasma only spends a fraction of its time in the cascade phase $\left(t_{\text {cascade }} \sim 10 \mathrm{~ns}\right.$, based on the average of the models), the reaction rates were scaled to the half period of a $30 \mathrm{kHz}$ sin wave $\left(T_{30 \mathrm{kHz}}\right)$, thus giving a scaling factor $\left(L=t_{\text {cascade }} / 2 T_{30 k H z}\right)$ of 0.0006 . The scaling factor allows direct comparison of reaction rates during the cascade phase to those in the afterglow and neutral phases.

Charge exchange rates also occur at very fast time scales and play a major role in the dynamics of charged particle populations and reactions during the cascade phase, thus they were also included in the cascade reaction rates. In the case of charge exchange, $v_{e}$ in Equation 2.23 was replaced with the ion velocity, $v_{i}$. The ions were assumed to be room temperature and to have a Boltzmann distribution, as they are not significantly accelerated by the electric field. Ionization cross-sections and energies (between 12-16 eV) for all species are relatively similar, therefore it is reasonable to assume that ion populations would initially be distributed based on each species' density with a total ion population equivalent to the electron density. Note, cross-sections for $\mathrm{CO}_{2}^{+}-\mathrm{CO} / \mathrm{CO}_{2}-\mathrm{CO}^{+}$and $\mathrm{CO}^{+}-\mathrm{O}_{2}$ (all are exothermic or energetically neutral) are not available in the literature and therefore were not plotted. However, due to the similar ionization energies of $\mathrm{CO}_{2}$ and $\mathrm{CO}$ their charge exchange rate is expected to be relatively small and ignorable. A similar difference in ionization energy between $\mathrm{CO}-\mathrm{O}_{2}$ and $\mathrm{CO}_{2}-\mathrm{O}_{2}$ implies that their cross-sections should be similar, therefore $\mathrm{CO}^{+}-\mathrm{O}_{2}$ should scale with the ratio of $n_{C O} / n_{C_{2}}$. For these experiments, this ratio never exceeds $10 \%$. 


\subsubsection{The Afterglow Phase and Neutral Phase}

After the cascade phase, the electric field is shielded, but the plasma persists for up to several milliseconds in the form of an afterglow $[66,73]$. At this point, the large population of electrons and ions created during the cascade phase have already begun thermalizing and recombining at a rapid rate, thus the afterglow phase is initially dominated by recombination reactions. The dissociations during the cascade phase combined with the recombination reactions create a large population of moderately energized free radicals, which are the dominant reactant species at this time.

The neutral phase occurs after the afterglow phase, when the streamer is cold (slightly warmer than room temperature) and the electron, ion, and radical populations are very small. The streamer at this point is equivalent to a thermal gas. Since $\mathrm{CO}_{2}$ dissociation is highly endothermic and the reverse reactions are exothermic, the neutral phase favors reverse reactions. Therefore, it is important to reduce the amount of time the plasma spends within the neutral phase if highly endothermic reactions are wanted. The easiest way to reduce a DBD's time within the neutral phase is to increase the driving frequency. This work, Paulssen and Wang all show that frequencies in the range of 30-60 kHz lead to more efficient dissociation $[15,18]$. These frequencies give a half period of $8.3-16 \mu \mathrm{s}$, much shorter than the afterglow lifetime. However, the neutral phase reactions still need to be considered during the lifetime of the streamer, because while the neutral reactions are only dominant during the neutral phase, they are still occurring during both the cascade and afterglow phases. This overlap in reaction rates is similarly true with the afterglow phase, which is considered to overlap the cascade phase. Because both the afterglow and neutral phases occur throughout the half-period of the discharge no scaling factor was needed for their

reaction rates. Since this also means the afterglow and neutral reactions occur within the 
same time frame of the discharge they are plotted together.

The free radical, recombination and neutral reaction rates for gas temperatures $\left(T_{g}\right)$ between 300 and $3000 \mathrm{~K}$ were solved using the rate equations

$$
r=\frac{d n}{d t}=k\left[R_{1}\right]\left[R_{2}\right]
$$

for 2 nd order reactions (2 reactants) and

$$
r=\frac{d n}{d t}=k\left[R_{1}\right]\left[R_{2}\right]\left[R_{3}\right]
$$

for 3 rd order reaction (3 reactants). $k$ is the rate coefficient and $\left[R_{1}\right],\left[R_{2}\right]$ and $\left[R_{3}\right]$ are the molar densities of reactants 1,2 and 3. A list of the relevant reactions and corresponding rate coefficients is in Table 2.6. $\mathrm{CO}_{2}, \mathrm{CO}, \mathrm{O}_{2}$ and $\mathrm{Ar}$ densities were scaled to atmospheric pressure densities $\left(n=2.454 \times 10^{19} \mathrm{~cm}^{-3}\right)$ based on their individual partial pressures. A density of $n_{e}=10^{12} \mathrm{~cm}^{-3}$ was used to show the minimal electron reaction rates. $\mathrm{CO}_{2}^{+}, \mathrm{CO}^{+}$ and $\mathrm{O}_{2}^{+}$densities were scaled to the electron density based on their partial pressures. Ar $^{+}$ densities were considered negligible due to the large charge exchange rates found during the cascade phase. O densities were $3.43 \times 10^{14} \mathrm{~cm}^{-3}$ as this is the minimum number of $\mathrm{O}$ that are needed for $\sim 5 \%$ dissociation in VADER at $200 \mathrm{SCCM}$ at $30 \mathrm{kHz}\left(4 \times 10^{-6} \mathrm{~mol} / \mathrm{s}\right)$, therefore reaction rates including $\mathrm{O}$ are minimum reaction rates. Ozone was not detected in any of the VADER experiments. However, ozone quickly breaks down even at low temperatures and ozone was found to be a favorable reaction even with minimal amounts of atomic oxygen. Therefore, the ozone density was scaled to the ozone production rate $\left(\mathrm{O}_{2}+\mathrm{O}+\mathrm{M} \rightarrow \mathrm{O}_{3}+\mathrm{M}\right)$ and the driving frequency, giving an ozone density of $\sim 10^{13} \mathrm{~cm}^{-3}$. 
Table 2.6: Afterglow/neutral Reactions and Rate Coefficients - The rate coefficients were solved using, $k=A\left(T_{g} / 300\right)^{B} e^{-C / T_{g}}, T_{g}$ is the gas temperature. $A$ has units of $\mathrm{cm}^{3} / \mathrm{mol} \mathrm{s}$ for 2nd order reactions and $\mathrm{cm}^{6} / \mathrm{mol}^{2} \mathrm{~s}$ for 3rd order. $C$ has units of Kelvin. Reaction rate constants with multiple sources were calculated using the NIST chemical kinetics database three parameter fit [79].

\begin{tabular}{|c|c|c|c|c|c|c|c|}
\hline Chemical Equation & Order & $\begin{array}{l}\min T_{g} \\
(\mathrm{~K})\end{array}$ & $\begin{array}{l}\max T_{g} \\
(\mathrm{~K})\end{array}$ & A & B & $\mathrm{C}$ & Source \\
\hline $\mathrm{CO}_{2}^{+}+\mathrm{O}_{2} \rightarrow \mathrm{CO}_{2}+\mathrm{O}_{2}^{+}$ & 2 & 300 & 10000 & $6.02 \mathrm{E}+13$ & 0.00 & $0.00 \mathrm{E}+0$ & [80] \\
\hline $\mathrm{CO}_{2}^{+}+e \rightarrow \mathrm{CO}+\mathrm{O}$ & 2 & 300 & 10000 & $3.91 \mathrm{E}+17$ & -0.80 & $0.00 \mathrm{E}+0$ & {$[53]$} \\
\hline $\mathrm{CO}^{+}+e \rightarrow C+O$ & 2 & 100 & 3000 & $1.66 \mathrm{E}+17$ & -0.55 & $0.00 \mathrm{E}+0$ & {$[81,82]$} \\
\hline $\mathrm{O}_{2}^{+}+e \rightarrow O+O$ & 2 & 100 & 5000 & $1.17 \mathrm{E}+17$ & -0.66 & $0.00 \mathrm{E}+0$ & {$[83,84]$} \\
\hline$O+O+M \rightarrow O_{2}+M$ & 3 & 200 & 4000 & $1.89 \mathrm{E}+13$ & 0.00 & $-9.00 \mathrm{E}+2$ & {$[85]$} \\
\hline $\mathrm{O}+\mathrm{CO}+\mathrm{M} \rightarrow \mathrm{CO}_{2}+\mathrm{M}$ & 3 & 300 & 2500 & $6.16 \mathrm{E}+14$ & 0.00 & $1.51 \mathrm{E}+3$ & [85] \\
\hline $\mathrm{O}+\mathrm{CO}_{2} \rightarrow \mathrm{O}_{2}+\mathrm{CO}$ & 2 & 300 & 2500 & $1.70 \mathrm{E}+13$ & 0.00 & $2.65 \mathrm{E}+4$ & [85] \\
\hline $\mathrm{O}_{2}+\mathrm{CO} \rightarrow \mathrm{O}+\mathrm{CO}_{2}$ & 2 & 300 & 2500 & $2.53 \mathrm{E}+12$ & 0.00 & $2.41 \mathrm{E}+4$ & [85] \\
\hline $\mathrm{O}_{2}+\mathrm{O}+\mathrm{M} \rightarrow \mathrm{O}_{3}+M$ & 3 & 100 & 3000 & $6.31 \mathrm{E}+13$ & -1.20 & $-2.89 \mathrm{E}+2$ & [86] \\
\hline$O_{2}+M \rightarrow O+O+M$ & 2 & 300 & 2500 & $6.08 \mathrm{E}+15$ & -1.00 & $5.94 \mathrm{E}+4$ & [85] \\
\hline $\mathrm{O}_{3} \rightarrow \mathrm{O}_{2}+\mathrm{O}$ & 2 & 300 & 3000 & $4.31 \mathrm{E}+14$ & 0.00 & $1.12 \mathrm{E}+4$ & {$[87]$} \\
\hline $\mathrm{O}_{3}+\mathrm{O} \rightarrow 2 \mathrm{O}_{2}$ & 2 & 200 & 2000 & $4.64 \mathrm{E}+12$ & -0.10 & $2.02 \mathrm{E}+3$ & {$[88,89]$} \\
\hline $2 \mathrm{O}_{3} \rightarrow 3 \mathrm{O}_{2}$ & 2 & 393 & 443 & $4.50 \mathrm{E}+12$ & 0.00 & $2.26 \mathrm{E}+2$ & {$[90]$} \\
\hline $\mathrm{CO}+\mathrm{O}_{3} \rightarrow \mathrm{CO}_{2}+\mathrm{O}_{2}$ & 2 & 296 & 301 & $2.41 \mathrm{E}-01$ & 0.00 & $0.00 \mathrm{E}+0$ & {$[91-93]$} \\
\hline$C O+M \rightarrow C+O+M$ & 2 & 5500 & 9000 & $9.15 \mathrm{E}+19$ & -3.10 & $1.29 \mathrm{E}+5$ & {$[94]$} \\
\hline
\end{tabular}

\subsubsection{Pure $\mathrm{CO}_{2}$ Plasma Results}

Figure 2.10 shows the reaction rates of a pure $\mathrm{CO}_{2}$ plasma before any dissociation. These results are relevant for a DBD system that has gas residence times similar to the discharge time of the plasma and the very early stages of a slower discharge. The first thing to note is that the ionization rate is consistent with those of a typical streamer. A typical streamer electron density is between $10^{12}-10^{15} \mathrm{~cm}^{-3}$, which corresponds to an e-impact ionization rate of $\sim 10^{-8}-10^{-5} \mathrm{~mol} / \mathrm{cm}^{3} \mathrm{~s}$ during the sample VADER conditions. Graph $\mathrm{C}$ of Figure 2.10 shows the plasma needs an average electron temperature of $5-10 \mathrm{eV}$ for these ionization rates, well within typical streamer discharge temperatures.

In Graph B of Figure 2.10, the vibrational excitation has the highest single reaction 
rate in the plasma. However, it is unclear how much vibrational excitation contributes to $\mathrm{CO}_{2}$ dissociation rates in a DBD. This uncertainty is because dissociation due to vibrational excitation requires multiple high energy collisions (which would be difficult to decouple from other impact dissociation reactions) and the literature gives inconsistent values of vibrational relaxation rates under plasma conditions. The vibrational to translational (VT) relaxation rate constant from Plasma Chemistry by Fridman is

$$
k_{V T} \approx 6.022 \times 10^{13} e^{-72 / T_{g}^{1 / 3}} \mathrm{~cm}^{3} / \mathrm{mol} \mathrm{s}
$$

where $T_{g}$ is in $\mathrm{K}$. This reaction rate constant is consistent with other sources and is stated to be a relatively slow process [14,95-97]. However, calculating the relaxation rate using the relaxation rate coefficient, an atmospheric pressure background density $\left(n_{n}=4.06 \times\right.$ $10^{-5}$ moles $/ \mathrm{cm}^{3}$ ) and a vibrationally excited population of density $\sim 5 \times 10^{-9}$ moles $/ \mathrm{cm}^{3}$ per discharge (calculated from the vibrational reaction rates at $5 \mathrm{eV}$ in Figure 2.10 at $300 \mathrm{~K}$ ), gives a VT reaction rate of $2.6 \times 10^{-4} \mathrm{~mol} / \mathrm{cm}^{3} \mathrm{~s}$. This means the $\mathrm{CO}_{2}$ molecules vibrationally relax at approximately the same rate as they are excited $\left(3 \times 10^{-4} \mathrm{~mol} / \mathrm{cm}^{3}\right.$ s versus $2.6 \times 10^{-4}$ $\left.\mathrm{mol} / \mathrm{cm}^{3} \mathrm{~s}\right)$. Similar simulations by Aerts et al. show that vibrational excitation is only a minor reaction when dissociating $\mathrm{CO}_{2}$ in a DBD [36]. This inconsistency in the effectiveness of vibrational excitation requires that other reaction pathways be considered during the analysis of dissociation rates.

The relatively fast $\mathrm{CO}_{2}$ dissociation rates consist of $\mathrm{CO}_{2}$ recombination $\left(\mathrm{CO}_{2}^{+}\right.$Recomb), $\mathrm{CO}_{2}$ dissociative attachment $\left(\mathrm{CO}_{2} \mathrm{Att}\right)$ and e-impact dissociation $\left(\mathrm{CO}_{2}\right.$ e-Impact $)$, as seen in Graph A of Figure 2.10. Each reaction has a strong temperature dependency, based on the energy of the reaction. The large energies needed for e-impact dissociation $(12 \mathrm{eV}$, 
$\mathrm{CO}_{2}$ Diss $)$, require $T_{e}>3 \mathrm{eV}$. At moderate plasma temperatures $\left(T_{e}>5 \mathrm{eV}\right)$ e-impact dissociation is the dominant reaction. Dissociative attachment requires less energy $(3.4 \mathrm{eV})$. Therefore, it occurs when $T_{e}>1 \mathrm{eV}$ and dominates the reaction rates between $2 \mathrm{eV}<T_{e}<6$ eV. Dissociative recombination occurs at all temperatures and becomes even more important at higher electron densities, because it scales with $n_{e}^{2}$ (assuming quasi-neutrality). All other e-collision reactions scale with $n_{e}$. The lack of an activation energy means dissociative recombination occurs faster at lower electron energies, between $0 \mathrm{eV}<T_{e}<4 \mathrm{eV}$. However, the reaction rate of dissociative recombination is very slow in comparison to other reactions, therefore while it is the dominant at low temperatures the rate is too low to have an effect on the chemistry. Therefore, it is proposed that the combination of e-impact dissociation, dissociative attachment and vibrational excitation are the main causes for $\mathrm{CO}_{2}$ dissociation during the cascade phase of the initial streamer breakdown. 


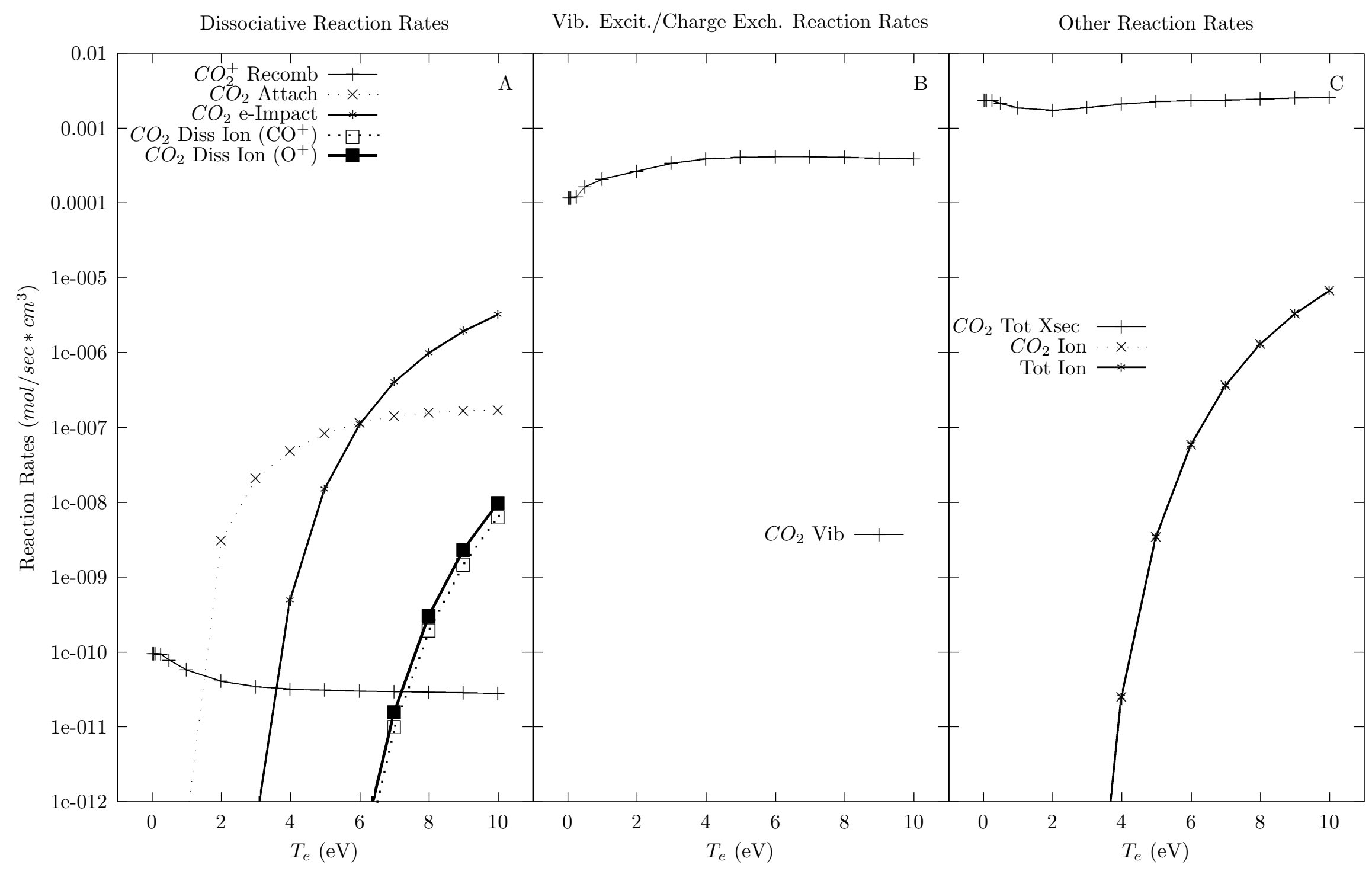

Figure 2.10: Cascade Phase Reaction Rates - 100\% $\mathrm{CO}_{2}$ before significant dissociation 
The afterglow/neutral phase reactions following a pure $\mathrm{CO}_{2}$ cascade phase are shown in Figure 2.11. The reactions that dominate during this low temperature phase are recombination reactions. $\mathrm{CO}_{2}^{+}$recombination occurs quickly even at low electron and ion densities (much faster at higher densities) and, due to the limited population of electrons and ions, recombination quickly replaces the electron population with an equivalent population of $\mathrm{O}$ and CO. A density of $3.43 \times 10^{14} \mathrm{~cm}^{-3}$ was used, because that is the minimum density of $\mathrm{O}$ and $\mathrm{CO}$ created per discharge for $5 \%$ dissociation in VADER. Depending on the gas temperature, the reactions will then either proceed through O-O bonding $\left(\mathrm{O}+\mathrm{O}+\mathrm{M} \rightarrow \mathrm{O}_{2}+\mathrm{M}\right.$, $\left.T_{g}<1200 \mathrm{~K}\right)$, CO oxidation $\left(O+C O+M \rightarrow C O_{2}+M, 500 \mathrm{~K}<T_{g}<1800 \mathrm{~K}\right)$, or $\mathrm{CO}_{2}$ reduction $\left(\mathrm{O}+\mathrm{CO}_{2} \rightarrow \mathrm{O}_{2}+\mathrm{CO}, \mathrm{T}_{g}>1300 \mathrm{~K}\right)$. CO oxidation is the reverse $\mathrm{CO}_{2}$ dissociation reaction, therefore during plasma startup it is important that the plasma is either less than $500 \mathrm{~K}$ or greater than $1500 \mathrm{~K}$ to ensure that the reverse reaction isn't encouraged. However, a higher temperature system also requires fast quenching, in order to reduce the amount of time is spent at temperatures where $\mathrm{CO}$ oxidation dominates. It is also important to note that the reaction rates for both $\mathrm{O}-\mathrm{O}$ bonding and $\mathrm{CO}$ oxidation are relatively slow, which should reduce the needed quenching rates. 


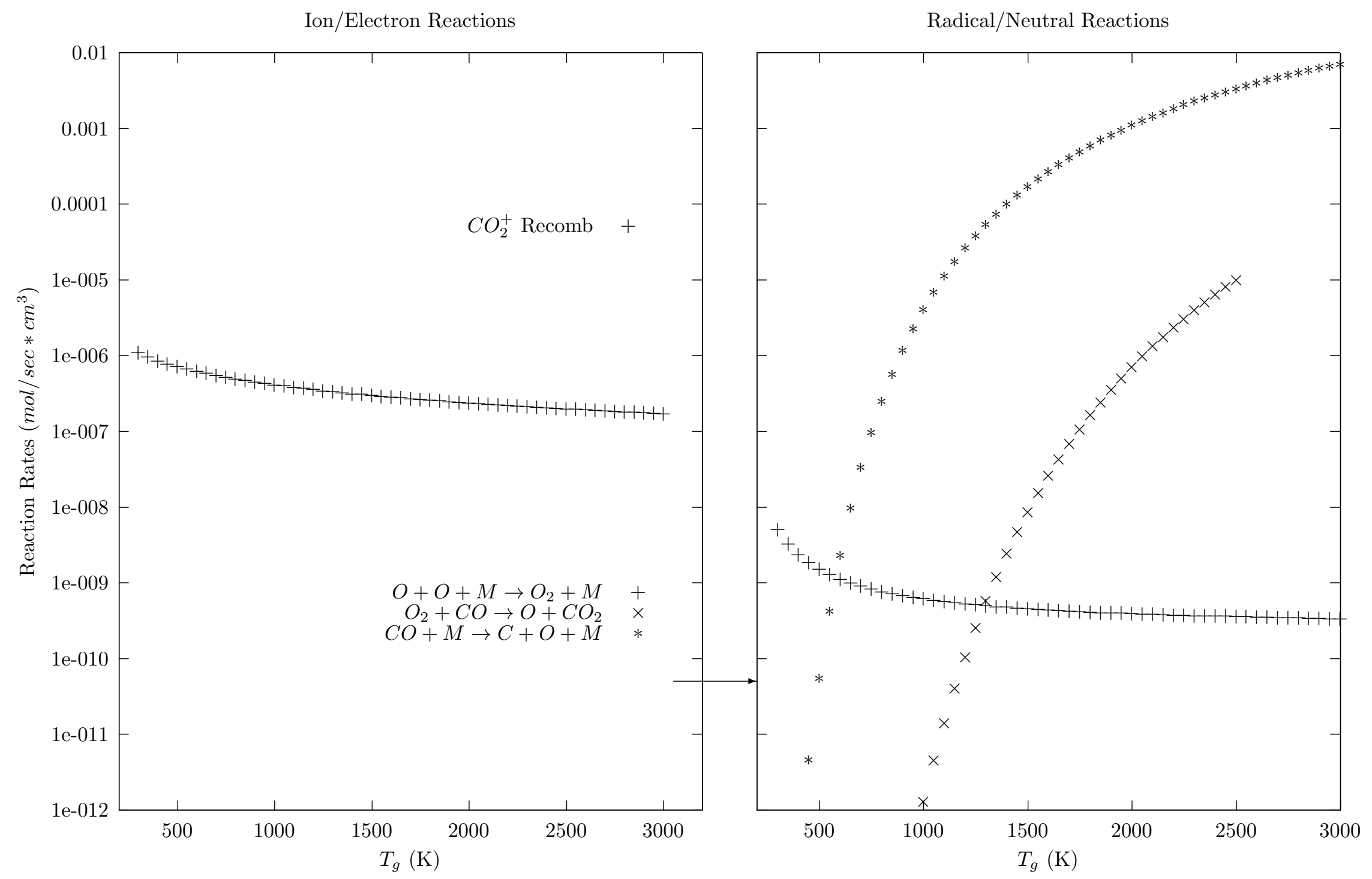

Figure 2.11: Afterglow/Neutral Phase Reaction Rates - $100 \% \mathrm{CO}_{2}$ before significant dissociation 


\subsubsection{5\% $\mathrm{CO}_{2} / 5 \%$ CO Plasma Results}

The residence time of gas within a streamer in VADER is several milliseconds, assuming a flow rate of $200 \mathrm{SCCM}$, a $0.3175 \mathrm{~cm}^{2}$ inlet cross-section and an average filament radius of $10^{-3}-10^{-4} \mathrm{~m}$. If the power supply driving frequency is $30 \mathrm{kHz}$, a volume of gas entering a streamer will go through hundreds of discharge cycles before leaving and many more times as the gas flows through different streamers in the reaction chamber. After multiple voltage cycles, a significant population of $\mathrm{CO}$ and $\mathrm{O}_{2}$ will have formed in the gas. This complicates the reaction kinetics as $\mathrm{O}_{2}, \mathrm{CO}$, and ozone reactions become more relevant to the plasma chemistry. To simulate the chemistry after multiple discharges, the calculations were repeated with $5 \%$ of the $\mathrm{CO}_{2}$ replaced with a proportional population of $\mathrm{CO}$ and $\mathrm{O}_{2}$ (in accordance with Equation 2.5).

The cascade phase reaction rates with $5 \% \mathrm{CO}$ are shown in Figure 2.12. Graph $\mathrm{C}$ shows that the addition of $\mathrm{CO}$ and $\mathrm{O}_{2}$ has a minimal effect on streamer ionization rates; this is because ionization energies of $\mathrm{CO}$ and $\mathrm{O}_{2}$ are similar to that of $\mathrm{CO}_{2}$.

Graph A shows that the small change in the $\mathrm{CO}_{2}$ population has little effect on the e-impact dissociation rates for $\mathrm{CO}_{2}$. The only major change to the dissociation rates is the addition of $\mathrm{CO}$ electron attachment dissociation. $\mathrm{CO}$ dissociation is beneficial for the goal of $\mathrm{CO}_{2}$ removal from the environment, as it leaves a single carbon atom which is highly reactive and can easily be converted to value added chemicals. However, no raw carbon was found within the VADER reaction chamber. Due to carbon's high reactivity and the large number of highly excited species in the plasma it is proposed that carbon atoms are quickly oxidized by $\mathrm{CO}_{2}, \mathrm{O}_{2}$ and $\mathrm{O}$ to reform $\mathrm{CO}(\mathrm{O}+\mathrm{C}$ reaction rates in a plasma are not

available in literature) $[79,98,99]$. All of these reactions are exothermic and lead to either further dissociation $\left(\mathrm{CO}_{2}+\mathrm{C} \rightarrow 2 \mathrm{CO}\right)$, an increase in the O population $\left(\mathrm{C}+\mathrm{O}_{2} \rightarrow \mathrm{CO}+\mathrm{O}\right)$ 
or the reverse reaction $(C+O \rightarrow C O)$. The destruction of $\mathrm{O}_{2}$ to form $\mathrm{O}$ is not a favorable reaction because $\mathrm{O}$ readily oxidizes $\mathrm{CO}$, thus causing an increase in the reverse reaction rate. Because the dissociation of $\mathrm{CO}$ essentially takes energy that could be spent directly dissociating $\mathrm{CO}_{2}$ and will often decompose the final products, $\mathrm{CO}$ dissociation is considered to have a negative effect on $\mathrm{CO}_{2}$ dissociation in VADER. However, this won't necessarily be the case if the carbon created is used to create a value added chemical. Some other minor reactions of note are $\mathrm{O}_{2}^{+}$recombination $\left(\sim 5\right.$ times slower than $\mathrm{CO}_{2}^{+}$recombination), $\mathrm{CO}^{+}$ recombination ( $\sim 5$ times slower than $\mathrm{CO}_{2}^{+}$recombination) and $\mathrm{O}_{2}$ dissociative attachment ( $\sim 5$ times slower than $\mathrm{O}_{2}$ dissociative attachment) which similarly reduce the efficiency of $\mathrm{CO}_{2}$ dissociation in VADER, but could be beneficial for the formation of value added chemicals.

Graph B shows that $\mathrm{CO}$ and $\mathrm{O}_{2}$ are both efficient at absorbing electron energy in the form of vibrational energy, especially at electron temperatures below $\sim 2 \mathrm{eV}$. This absorption means less of the vibrational energy is going towards the dissociation of $\mathrm{CO}_{2}$ and is instead going towards $\mathrm{CO}$ and $\mathrm{O}_{2}$ dissociation. Both reactions create $\mathrm{O}$, which as discussed above does not benefit the dissociative process. $\mathrm{O}_{2}$ is especially effective at reducing dissociation rates due to its high charge exchange rate with $\mathrm{CO}_{2}^{+}$, which reduces $\mathrm{CO}_{2}^{+}$recombination rates and increases $\mathrm{O}_{2}^{+}$recombination. 


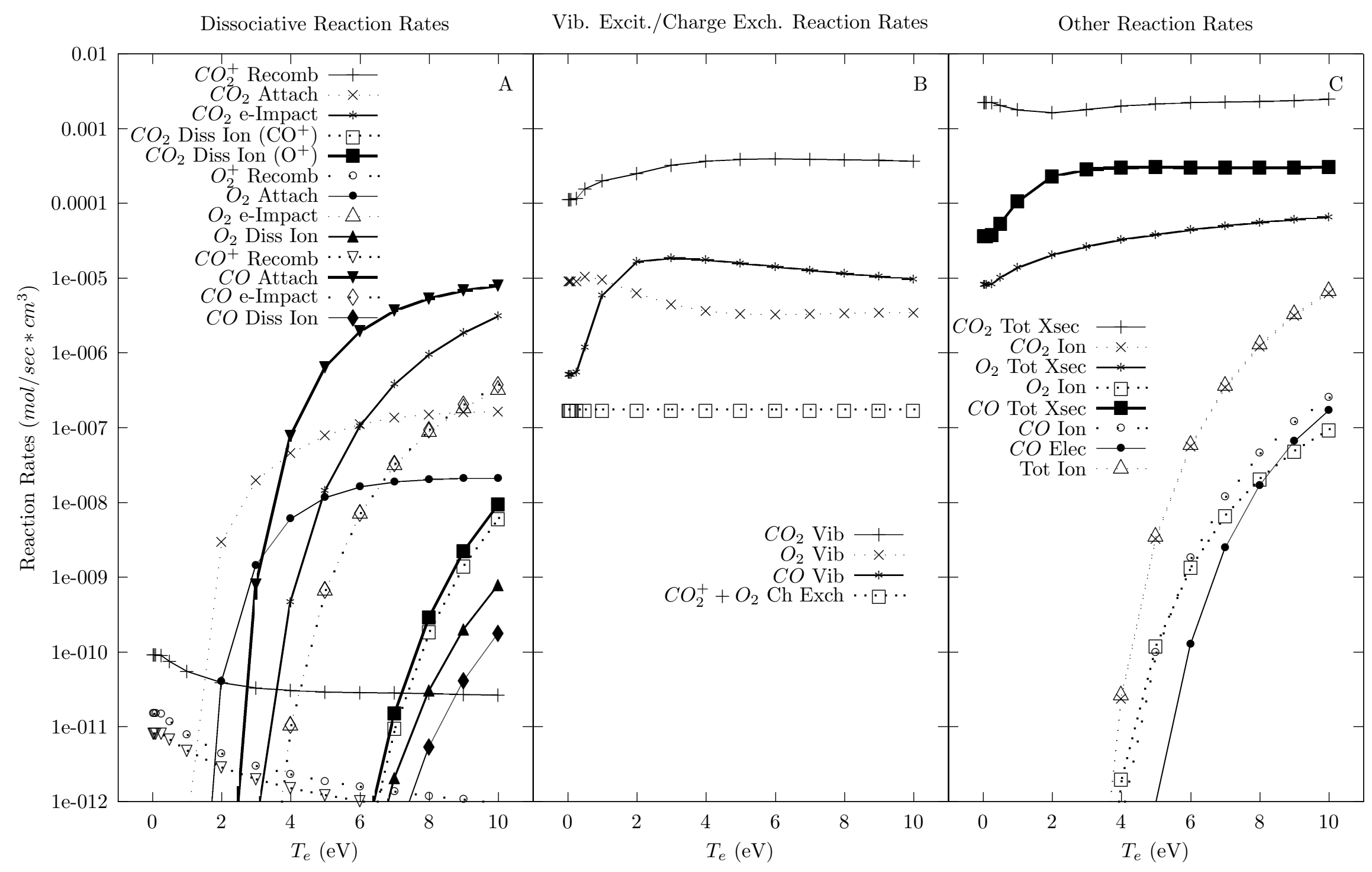

Figure 2.12: Cascade Phase Reaction Rates - 92.5\% $\mathrm{CO}_{2}, 5 \% \mathrm{CO}, 2.5 \% \mathrm{O}_{2}$ 
The afterglow/neutral reaction rates for the 5\% CO case are shown in Figure 2.13. The graphs show that once again $\mathrm{O}_{2}$ is responsible for slowing down $\mathrm{CO}_{2}$ dissociation rates in a DBD. Charge exchange between $\mathrm{CO}_{2}$ and $\mathrm{O}_{2}$ further reduces $\mathrm{CO}_{2}^{+}$recombination rates in exchange for $\mathrm{O}_{2}^{+}$recombination. At high temperatures $\left(\mathrm{T}_{g}>500 \mathrm{~K}\right), \mathrm{O}_{2}$ and $\mathrm{O}$ react quickly with $\mathrm{CO}$ forming $\mathrm{CO}_{2}$ with a small percent forming the final product of $\mathrm{O}_{2}$. Only at low temperatures $\left(\mathrm{T}_{g}<500 \mathrm{~K}\right)$ does $\mathrm{O}$ prefer to form ozone over $\mathrm{CO}_{2}$. If the ozone is then heated above $\sim 500 \mathrm{~K}$, it decays back into its constituents with no effect. However if the gas temperature is kept low $(<400 \mathrm{~K})$ ozone provides the only relatively quick path to $\mathrm{O}_{2}$ formation once a population of $\mathrm{O}_{2}$ has formed. This singular ozone path implies that low gas temperatures and cooling are necessary for efficient $\mathrm{CO}_{2}$ dissociation (similar to ozonizers). Also, it implies that oxygen content is one of the most important factors for determining the efficiency of $\mathrm{CO}_{2}$ dissociation. 
Ion/Electron Reactions

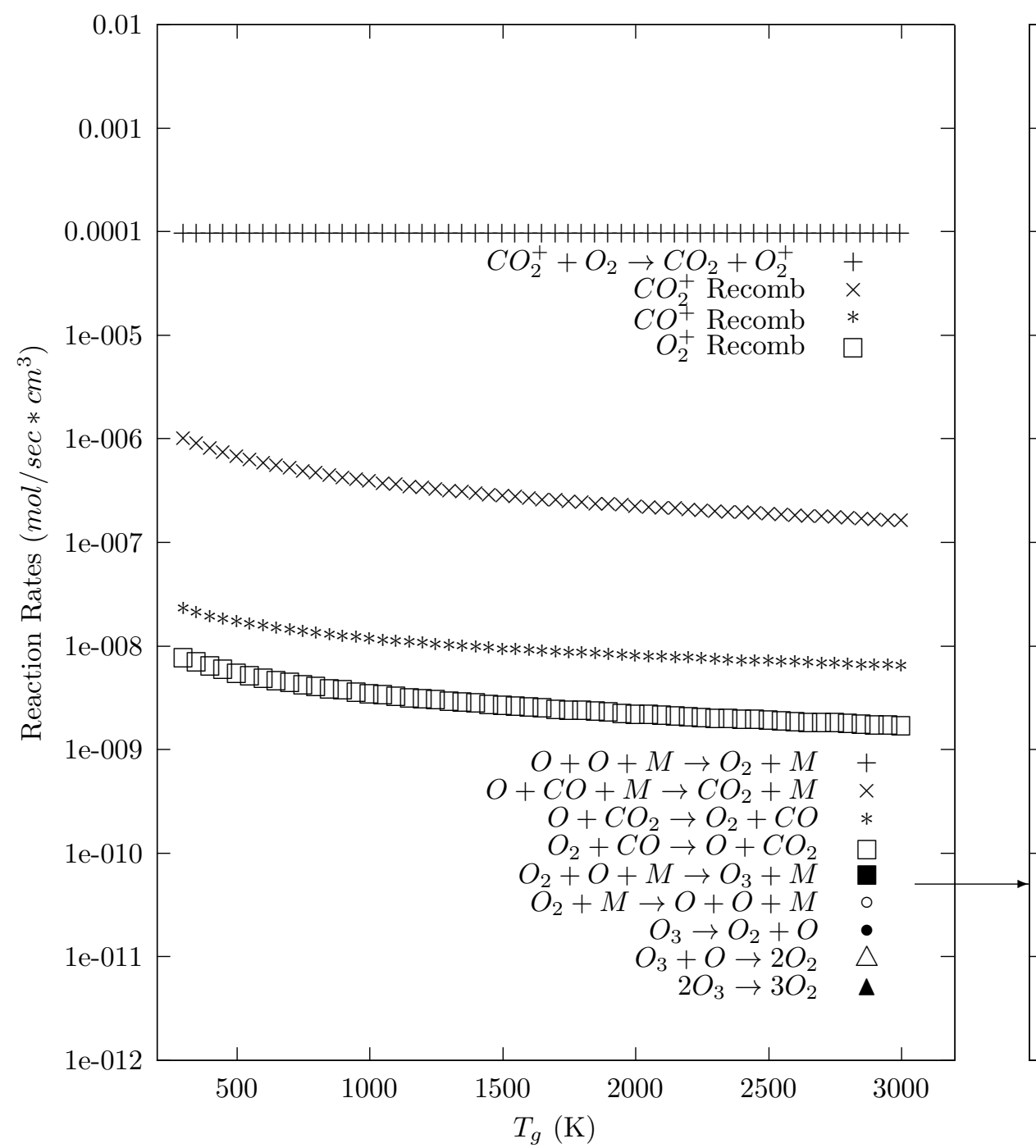

Radical/Neutral Reactions

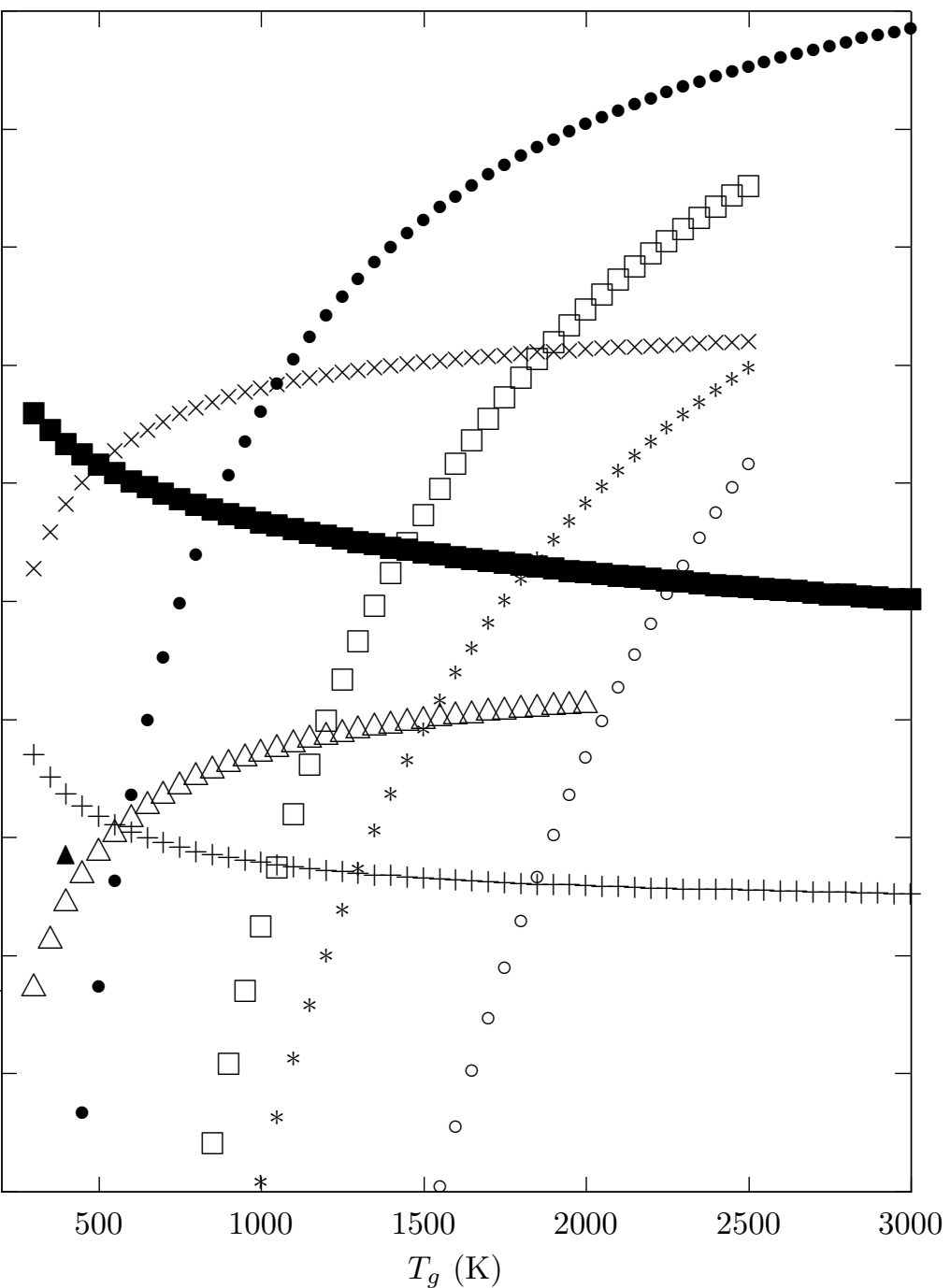

Figure 2.13: Afterglow/Neutral Phase Reaction Rates - 92.5\% $\mathrm{CO}_{2}, 5 \% \mathrm{CO}, 2.5 \% \mathrm{O}_{2}$ 


\subsection{5 $\quad 60 \% \mathrm{CO}_{2} / 40 \%$ Ar Plasma Results}

Figure 2.14 shows the results of the $95 \% \mathrm{CO}_{2} / 5 \% \mathrm{CO}$ reaction rate calculations with $40 \%$ of the $\mathrm{CO}_{2}, \mathrm{CO}$ and $\mathrm{O}_{2}$ replaced with argon. As discussed in Section 2.1.4 and as seen in graph $\mathrm{C}$, argon slightly decreases ionization rates and $\mathrm{Ar}^{+}$has a negligible recombination rate. The recombination rate has a maximum of $r=4 \times 10^{-14} \mathrm{~mol} / \mathrm{cm}^{3}$ s at $n_{e}=10^{12} \mathrm{~cm}^{-3}$ (not plotted) which scales with $n_{e}^{2}$. Graph B shows that the large argon charge exchange cross-sections create $\mathrm{CO}_{2}^{+}, \mathrm{CO}^{+}$and $\mathrm{O}_{2}^{+}$. The $\mathrm{Ar}^{+}-\mathrm{CO}_{2}$ charge exchange rates are 2 orders of magnitude higher than either $\mathrm{Ar}^{+}-\mathrm{CO}$ and $\mathrm{Ar}^{+}-\mathrm{O}_{2}$ charge exchange rates. Therefore, argon acts as a source of $\mathrm{CO}_{2}^{+}$ions, which can then go through recombination to increase dissociation rates. This is one possible explanation for the improved dissociation rates and efficiencies found in VADER when argon was added to the system.

The afterglow/neutral reaction rates with the addition of argon were shifted down by $40 \%$ and had no noticeable change from the results in Figure 2.13. 


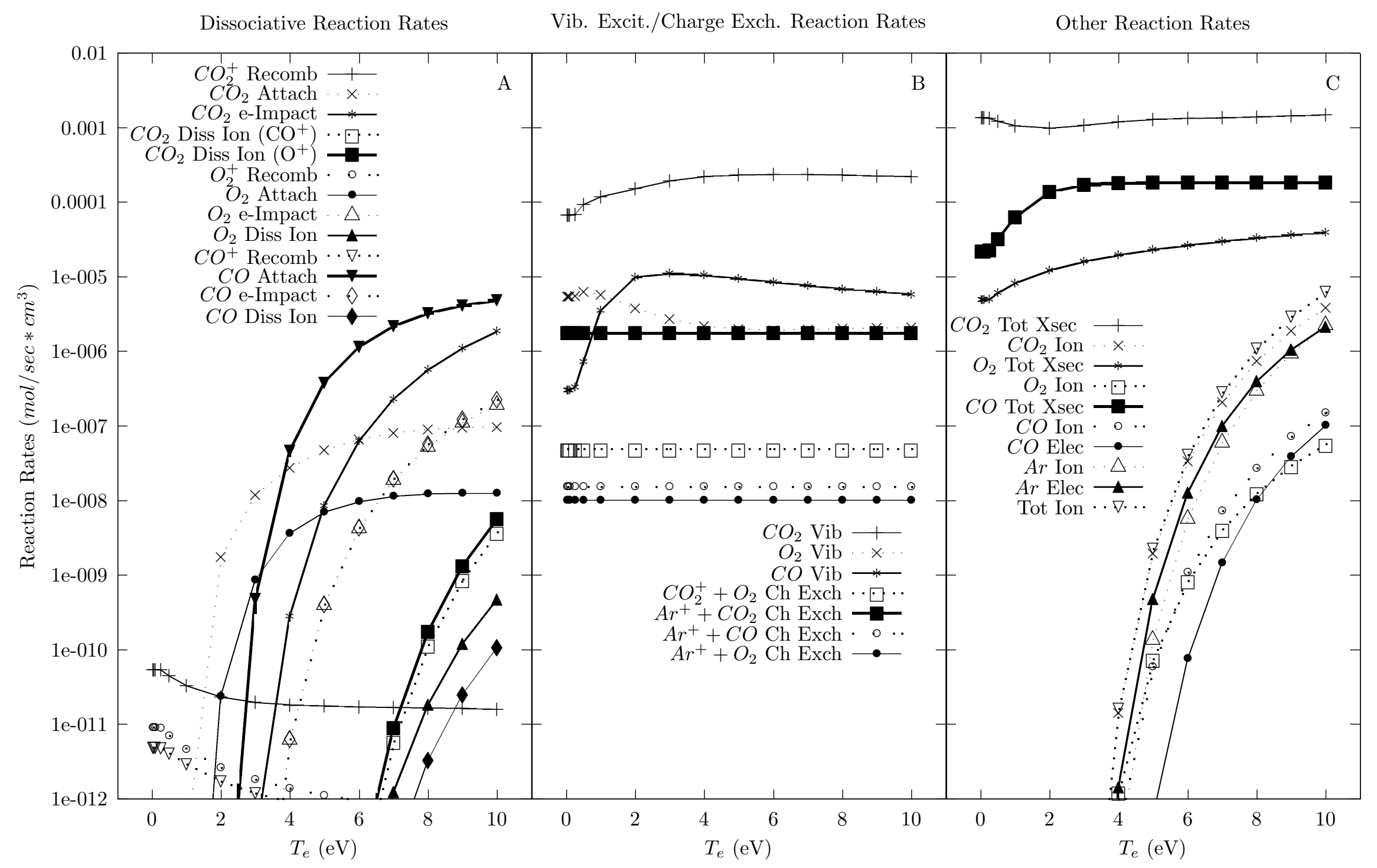

Figure 2.14: Cascade Phase Reaction Rates - $60 \% \mathrm{CO}_{2}, 40 \% \mathrm{Ar}, 3 \% \mathrm{CO}, 1.5 \% \mathrm{O}_{2}$ 


\subsubsection{Discussion}

The reaction rate calculations presented here provide a simplified perspective on localized reactions within the core of a streamer and do not take into account many of the complex dynamics and surface effects that occur within a DBD. These complex dynamics include streamer edge effects, energy transfer, photon emission, surface reactions and electric field compression.

As a volume of gas moves through a streamer, it first enters the streamer edge. The streamer edge is a lower density region of the streamer, which is generally accepted as a lower density and temperature region of the plasma. While it can be expected that the chemistry is similar to that of the bulk of the plasma, it is unclear how far this region of the plasma extends and what the effects of a gradient in plasma density and temperature have on the plasma chemistry.

Photoemission in a plasma is most often due to the relaxation of excited electronic and vibrational states. It has been proposed that these emissions play an important role in plasma breakdown, energy transfer and energy loss due to emission and re-absorption by the plasma [14]. The model used here assumes that photoemission has a negligible effect on the chemistry of the plasma. This assumption is based on the minimal amounts of emission that were observed with spectrometers during the VADER experiments and the inability to see any absorption during absorption spectroscopy experiments. While highly unlikely, the low amounts of photoemission could be due to the re-absorption of resonant emission lines within the plasma and surrounding gases, which would effectively cool the denser central plasma regions and heat the lower density surrounding regions.

As reported in this work and by others, surface materials have a significant effect on plasma chemistry within a DBD $[18,32,100]$. Therefore, a complete model of the surface 
chemistry is required for a complete model of the chemical kinetics within a DBD. The interaction between plasmas and surfaces are very complex, involving changes in surface chemistry due to the plasma, changes in plasma properties due to the surface material, the introduction of new species into the system, complex catalytic chemistry, various temperature effects, etc [44]. Creation of models including the surface chemistry are extremely difficult and were considered beyond the scope of this work.

Every collision, emission and chemical reaction within a plasma entails an energy transition, which includes photon emission, vibrational energy changes, rotational energy changes, electronic energy changes, etc. Depending on the species and the transition, the location of the energy can change the chemical dynamics of the plasma; for instance if a lot of energy is transferred into rotational energy then there is less vibrational energy available for dissociation. To include these kinds of interactions is computationally taxing and difficult and was thus not included in the model.

Electric field compression is discussed in Section 2.2.5. It is the increase in electric field between the cascade wavefront and the charged electrode as the wavefront moves closer to the electrode surface. Electric field compression creates an anisotropy in the plasma temperature between the electrodes. The chemical models shows that small changes in plasma temperature can have large effects on $\mathrm{CO}_{2}$ dissociation and can completely change the surface chemistry in the plasma. However, to include this anisotropy would require the code to evolve temporally, which was beyond the scope of this work.

The PLASMANT research group at the University of Antwerp is currently developing a much more comprehensive DBD chemistry model for $\mathrm{CO}_{2}$ dissociation [36]. 


\section{Chapter 3}

\section{VADER Design}

To look into the variables that affect $\mathrm{CO}_{2}$ dissociation within a DBD, the Versatile Atmospheric Dielectric Barrier Discharge Experiment (VADER) was designed. The VADER system allows for large amounts of flexibility in the DBD parameters including the ability to have variable gas flows, power supplies, electrode distances, dielectric materials, electrode orientations, temperatures, etc. To look into the chemistry and plasma physics of a DBD, VADER utilized power measurements (voltage dividers and rogowski coils), an atmospheric

pressure residual gas analyzer (RGA) system, and optical access within the near-infrared to near ultraviolet optical ranges.

\section{$3.1 \quad$ System Overview}

The Versatile Atmospheric Dielectric barrier discharge Experiment (VADER) is shown in Figure 3.1 and a block diagram of the system is shown in Figure 3.2. 


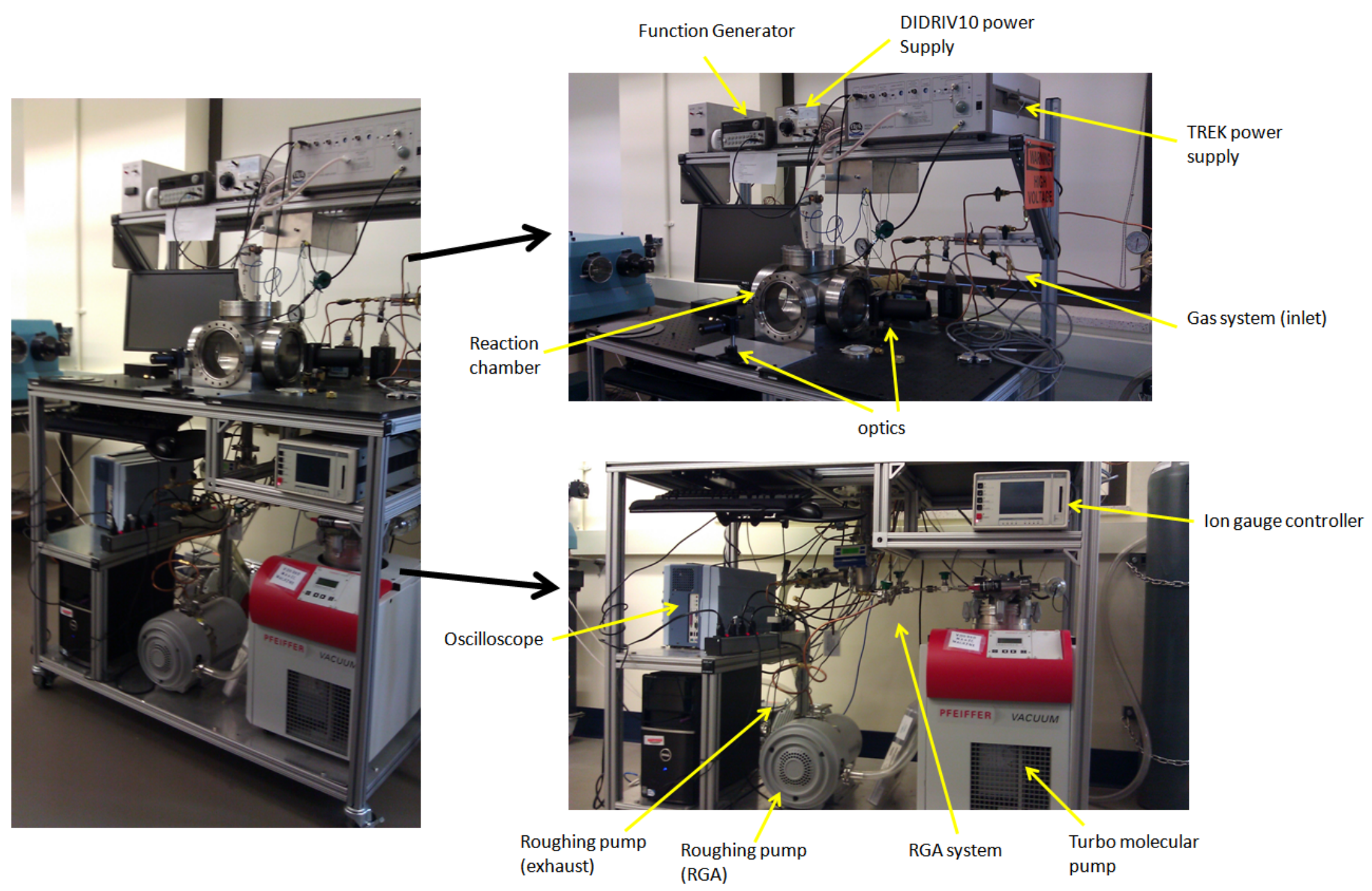

Figure 3.1: The VADER reactor 


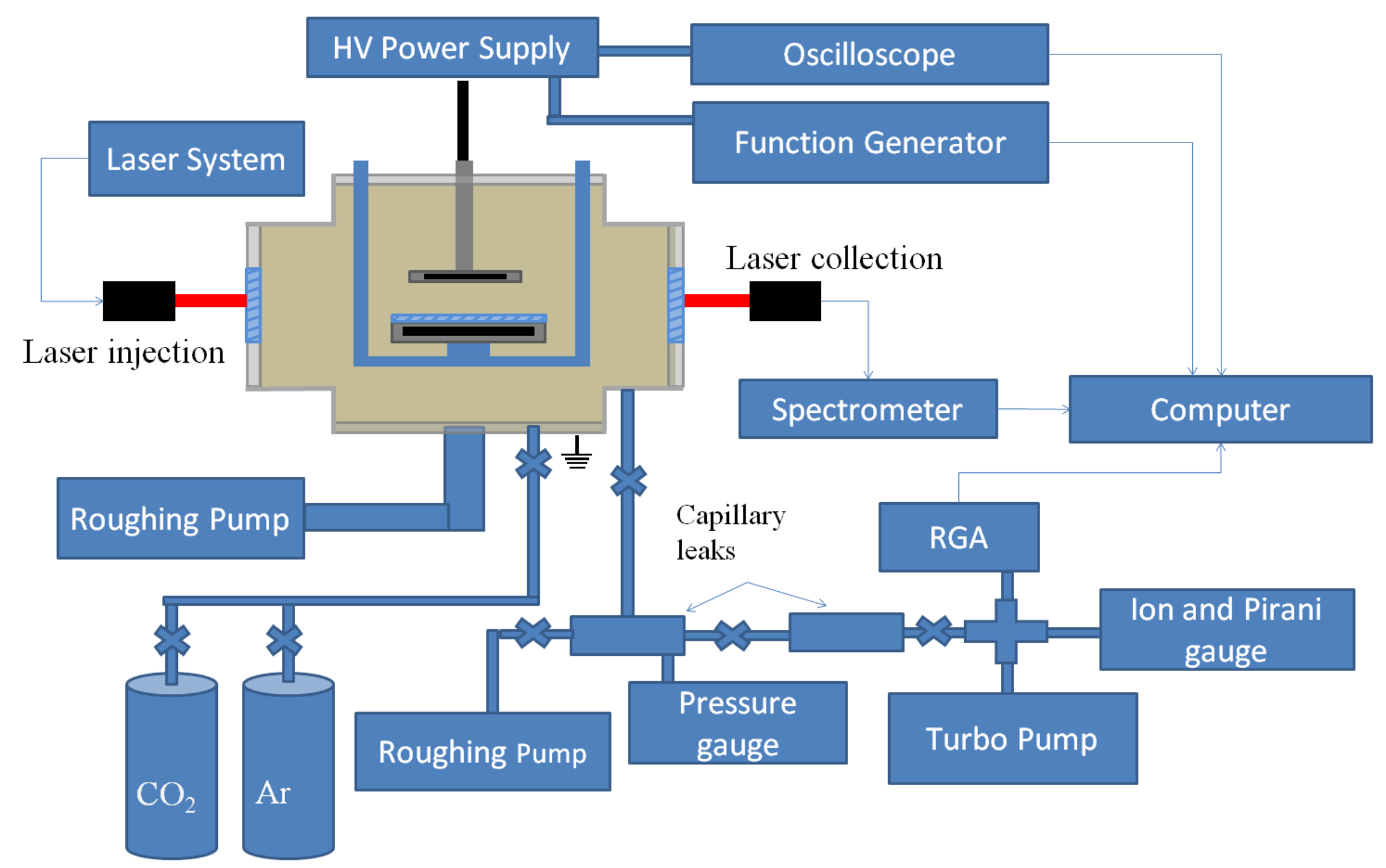

Figure 3.2: A schematic of the VADER system 


\subsubsection{Reaction Chamber}

The VADER reaction chamber, shown in Figure 3.3, consists of an 8" Lesker 6 way CF cross used to contain the plasma and reaction gases. One 8" flange was used for the HV feedthrough and ground line, one for gas feedthroughs, one for a roughing pump connection, and three were outfitted with optical viewports for plasma and optical emission spectroscopy. One of the optical viewports was a Lesker quick open flange for easy access to the DBD at the center of the chamber.

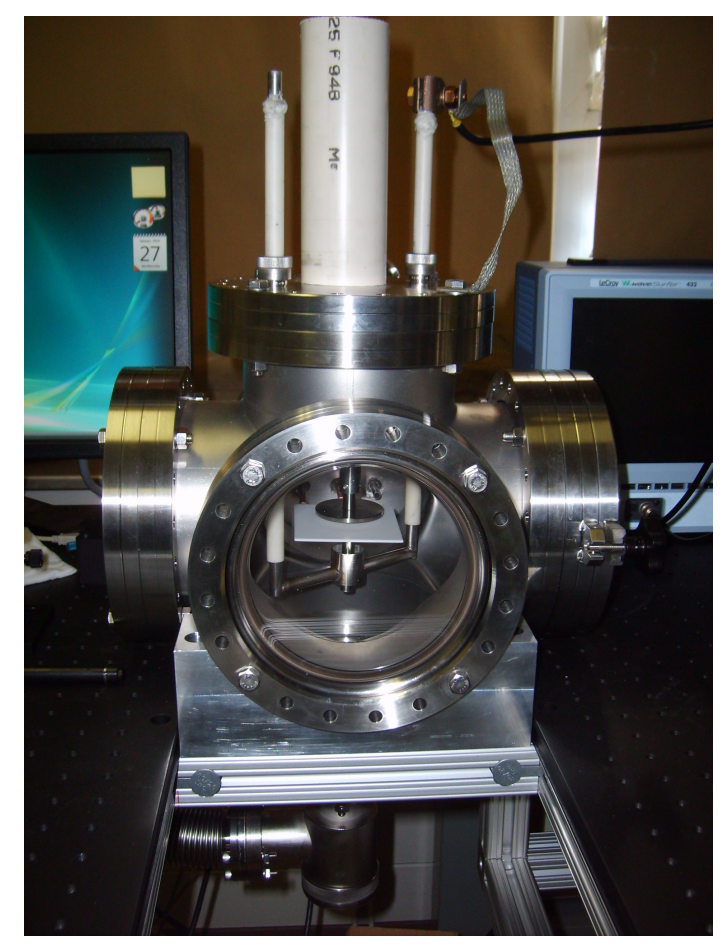

Figure 3.3: The VADER reaction chamber.

\subsubsection{High Voltage (HV) System}

Two HV systems were used in this experiment, one using a function generator connected to a Trek model 10/10B HV power amplifier (TREK supply) and the other a standalone 
DIDRIV10 power supply from amazing1.com.

The Trek supply was designed to supply $\pm 10 \mathrm{kV}$ between the frequencies of $0-10 \mathrm{kHz}$ with a max current of $10 \mathrm{~mA}$ and a max power of $50 \mathrm{~W}$ by amplifying an input signal by 1000:1. The input signal was supplied by a HP 33120A 15MHz Waveform Generator and connected to the front of the power supply using a standard BNC fitting. Several different waveforms, including square, triangular and sinusoidal were employed. The amplifier output used a custom high voltage lead which was connected directly to the HV feedthrough on the top flange. The power output for the TREK system was measured using the TREK power supply's built in voltage and current monitors. As is usual for high frequency amplifiers, the TREK system's voltage and power dropped off at higher frequencies, starting at $2.5 \mathrm{kHz}$ (see Figure 3.4). The upper range of frequencies during which plasmas would form was 4-7 $\mathrm{kHz}$ for most CO2 plasmas. Due to this frequency limitation, all runs using the TREK power supply were done with the highest voltage settings and the lower power supply frequencies. Also, the system's inductance at high frequency limited the bandwidth of the system and caused all waveforms above $2.5 \mathrm{kHz}$ to be sinusoidal.

The DIDRIV10 power supply had a variable voltage between $0 \%$ to $100 \%$ of the maximum voltage with a stated max voltage of $\pm 20 \mathrm{kV}$ and frequencies ranging from $28 \mathrm{kHz}$ and 64 $\mathrm{kHz}$ with a sinusoidal waveform. The specifications listed a maximum power of $200 \mathrm{~W}$, but when connected to the DBD system a maximum power of $\sim 85 \mathrm{~W}$ was measured. A severe drop in voltage and power was measured as frequency increased. Voltages for frequencies above $35 \mathrm{kHz}$ were so low that a plasma did not form. Therefore, all runs were done at $28.4 \mathrm{kHz}$ and $30 \mathrm{kHz}$. An example of the current, voltage, and associated power waveforms is shown in Figure 3.5. The current and voltage curves have large spikes after the voltage peaks (but not as the voltage increases) due to the bulk of the charge on the surface of the 


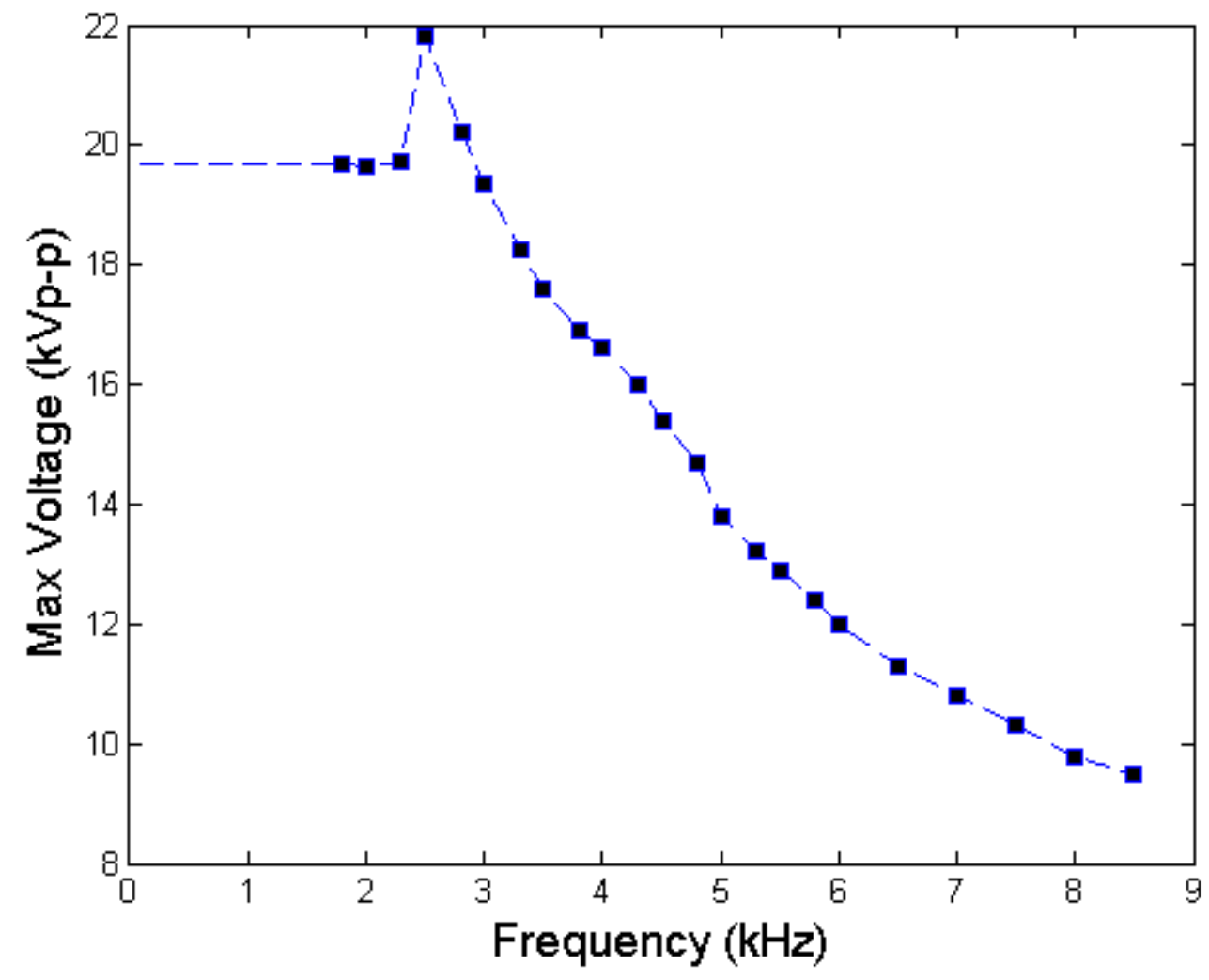

Figure 3.4: The Trek power supply's voltage dropoff versus frequency. The supply was driven with a constant amplitude square wave (20 Vp-p).

dielectric being repelled as voltage is reversed. These discharge characteristics have been seen in other DBDs [101].

The DIDRIV10 power supply has adjustable duty cycles which allow the power supply to be pulsed on and off at set time intervals. For example, one duty setting turned the power supply on for 3.6 milliseconds, then off for 0.5 milliseconds, then back on for $3.6 \mathrm{~ms}$, etc. The duty cycles were limited to a several millisecond (ms) range. Therefore, the duty cycles used were $3.6 \mathrm{~ms}$ on with $0.5 \mathrm{~ms}$ off and $2.4 \mathrm{~ms}$ on with $2.4 \mathrm{~ms}$ off. Examples of the current, voltage, and power profiles from the two duty cycles are shown in Figure 3.6.

The DIDRIV10 power supply did not include an internal method of finding the voltage and current (and in turn power), so a voltage divider and current monitor were attached to 

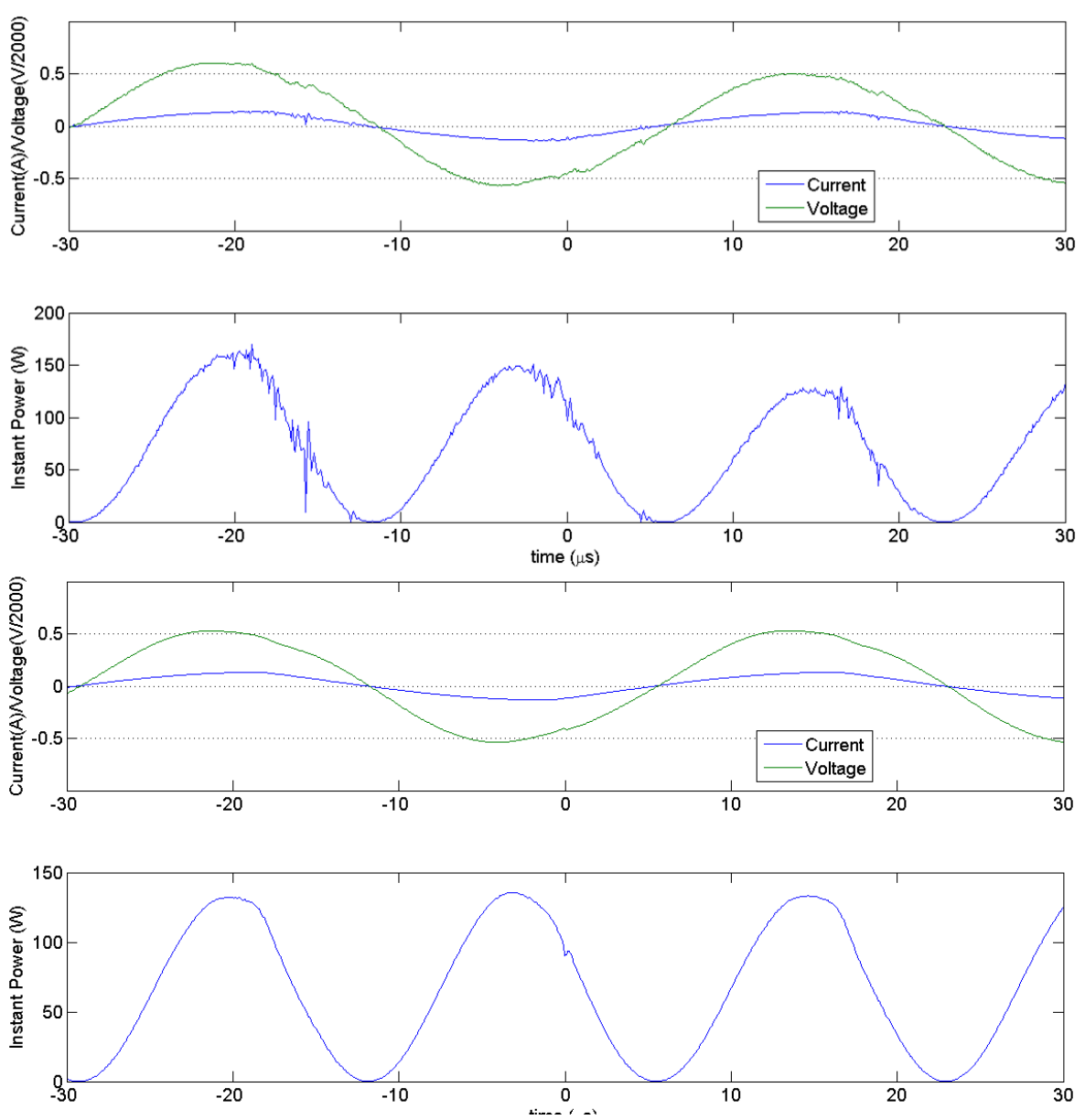

Figure 3.5: Example of current, voltage, and power time series used for VADER using the DIDRIV10 Power Supply. Each curve is from the same plasma with an average power of 70W. The top two curves are a case with no averaging, the second two curves are after 100 averages.

the system as discussed in Section 3.2.3. It was found that the DIDRIV10 power supply has an initial HV pulse when the plasma is turned on, after which the voltage drops to a constant amplitude, as is evident in Figure 3.6. The initial voltage spike was consistently $\sim 4 \mathrm{kV}$, whereas the continuous mode voltage depended on several experimental settings including frequency, gas composition and dielectric material. A maximum voltage and current of $\pm 1,900 \mathrm{~V}$ and $513 \mathrm{~mA}$ was found at $28.4 \mathrm{kHz}$ and $\pm 1,715 \mathrm{~V}$ and $455 \mathrm{~mA}$ at $30 \mathrm{kHz}$. The voltage scaled linearly as the voltage knob was swept from $0 \%$ to $100 \%$ voltage. Due to the 
variation in maximum voltages, all of the DIDRIV10 voltage readings are reported as either $100 \%$ (the voltage setting at maximum) or $90 \%$ (90\% of the max). At lower voltages, the power supply did not consistently produce a plasma.
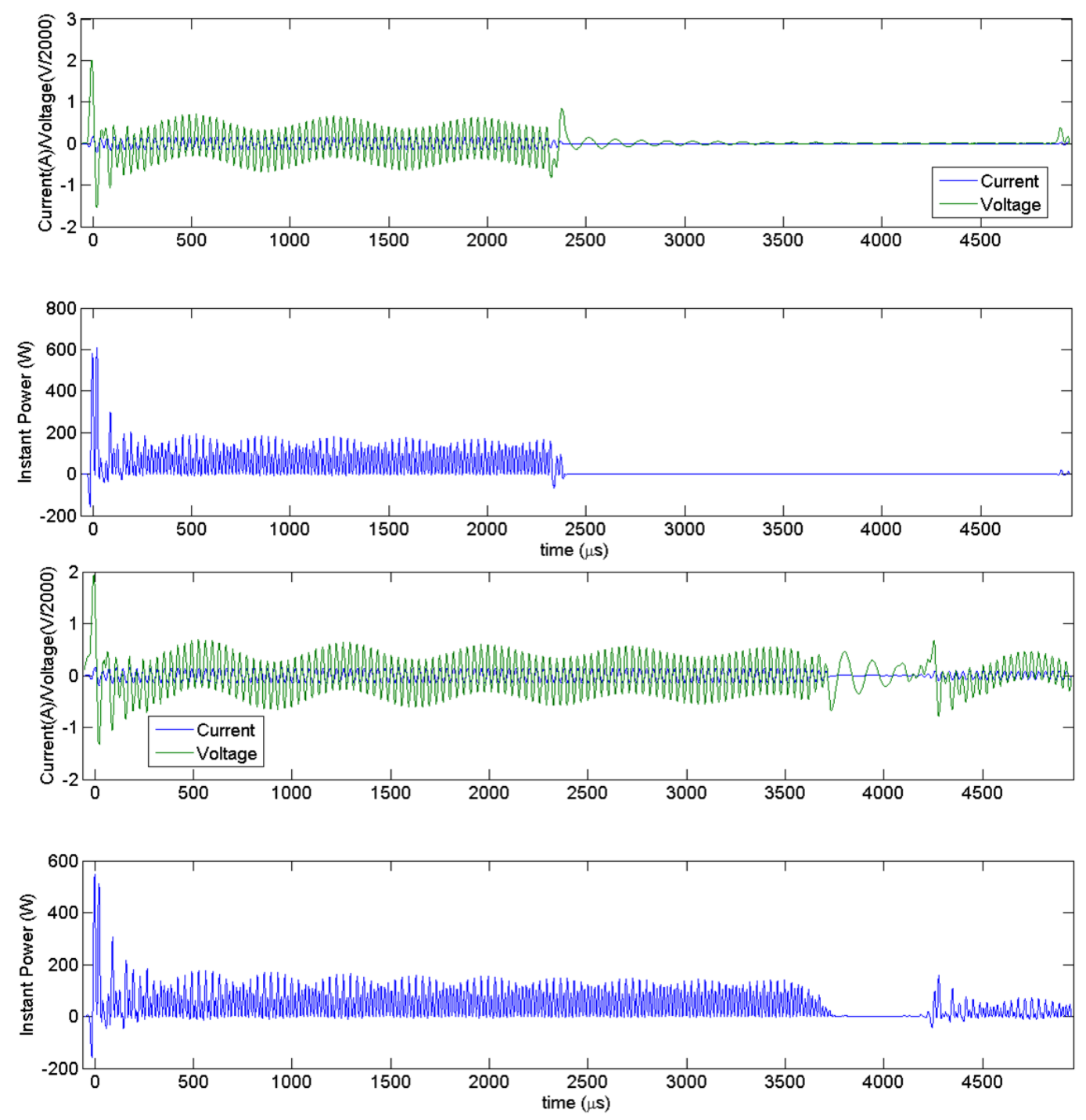

Figure 3.6: Examples of the current-voltage and power curves for two different duty cycles using the DIDRIV10 Power Supply. The top curves are for the $2.4 \mathrm{~ms}$ on with $2.4 \mathrm{~ms}$ off duty cycle and the bottom curves are for the $3.6 \mathrm{~ms}$ on with $.5 \mathrm{~ms}$ off duty cycle

The power supplies connected to a HV feedthrough flange, which consisted of an 8" blank flange modified to have a Lesker 30kV HV feedthrough (part number EFT3012091) welded to 
the center and two 1/2" Cajon fittings welded on to opposite sides of the feedthrough for use with a custom U-shaped basket. The U-shaped basket acted as the central grounding point for the system and held the ground electrode/dielectric box, see Figure 3.7. The connection point between the feedthrough and the HV line was insulated electrically and physically using two layers of PVC piping. The electrodes used in VADER were 3/8" stainless steel (SS) rods welded to 2" diameter, SS disks. To connect the HV electrode to the .094" SS rod coming from the feedthrough, a custom made SS connector was designed to accept the .094" HV feedthrough tip and the 3/8" SS rod, which were pinned in place using screws in the SS connector. The U-shaped basket consisted of a set of 3/8" SS rods welded into a U-shape with a $1 / 2$ " diameter alumina sheath Torr-sealed (TM) into place on both arms of the U. Once sealed, the 1/2" alumina sheathed arms were fed through the $1 / 2$ " Cajon (TM) fittings on the flange. The Cajon fittings held the basket in place and the Torr-sealed arms and fittings ensured the vacuum seal. At the bottom of the U-shaped basket a 3/8" inner diameter ring was welded in place, which also had a screw for clamping the ground electrode in place. The HV feedthrough flange had the HV electrode hanging within the center of the U-shaped basket over the ground electrode, leaving a large amount of space between the HV electrode and any non-DBD related metallic surfaces. This feedthrough design also allowed us to conveniently adjust the electrode gap without losing vacuum by loosening the Cajon fittings and either raising or lowering the height of the basket using the protruding basket arms. The main issue with the HV feedthrough was lining up the ground and HV electrodes, which is necessary for a uniform discharge. 


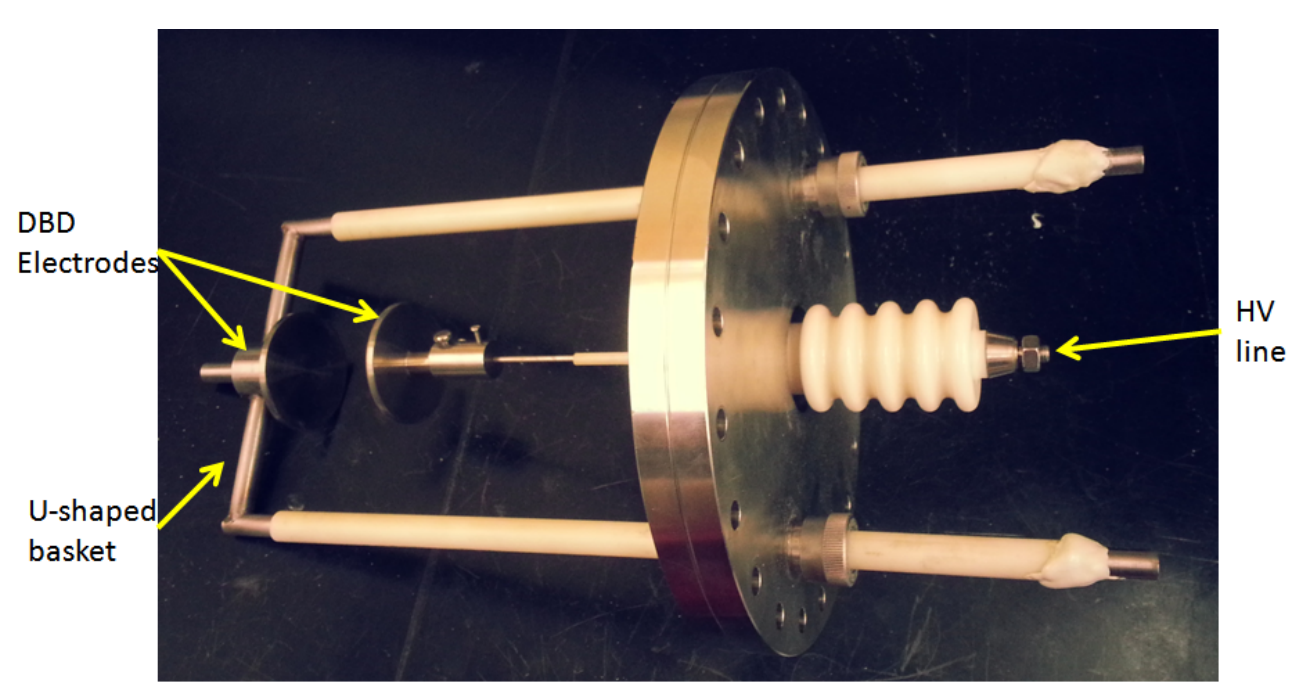

Figure 3.7: The HV feedthrough flange.

\subsubsection{The Reaction Box}

The reaction box was a small reaction chamber that, when sandwiched between the ground and HV electrodes, creates a planar DBD geometry-see Figure 3.8. After several iterations, the final design was made in three layers. The bottom was a machined stainless steel (SS) disk, the middle was the air gap with dielectric walls Torr-sealed in place and the top was a dielectric disk glued to the middle piece, giving a total reaction volume of $\sim 3.2 \mathrm{~cm}^{3}$. It included four ports. Three were used as a gas inlet, gas outlet and for a pressure gauge. The 4th port was capped. The reaction box was designed to alleviate issues arising from slightly misaligned electrodes, ensure the distance between electrodes were consistent for each run, and to constrain the volume of gas passing through the reaction zone during experimental runs.

The bottom layer of the reaction box was a thin 3" diameter SS disk with two 1/16" thick rings of diameter $21 / 4$ " and $23 / 4$ ", see Figure 3.8. The top surface of the cut disk section acted as one of the DBD electrodes. The middle layer sat in the groove of the first 


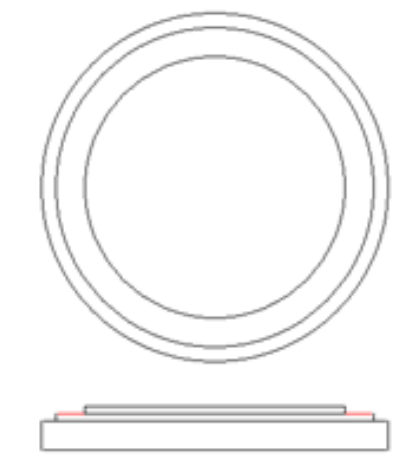

Bottom layer

3" diameter machined stainless steel disk (each cut is 1/16"deep)

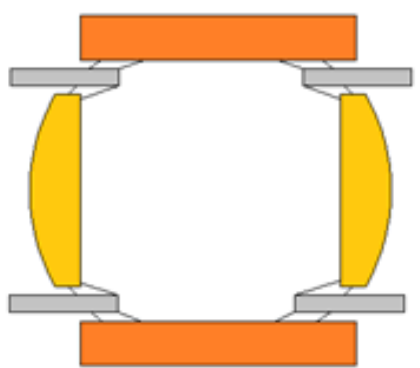

Polishedquartz Quartz Alumina tubes Torr-seal Middle layer Each piece is vacuum sealed to the bottom layer using Torrseal in the above configuration

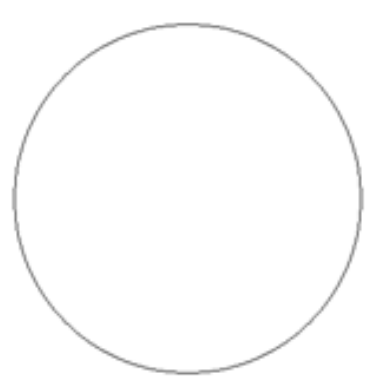

Top layer A 3" piece of quartzglass, sealed to the middle layer using crystalbond

Figure 3.8: The final reaction box design.

ring of the bottom layer and was a set of $1 / 8$ " thick quartz pieces, cut from a larger quartz disk, and short alumina tubes Torr-sealed into place. The middle layer created a 1/16" thick reaction zone. The thickness was selected based upon preliminary discharge tests and was adjustable by changing the groove depth or quartz thicknesses. The middle section acted as the walls of the system. The orientations of the gas inlet and outlet tubes were to ensure that the gas swirled through the plasma, maximizing the gas residence time in the reaction chamber. The second ring groove acted as a repository for the Torr-seal to form a vacuum seal. The Torr-seal groove was cut deep enough such that a Dremel (TM) tool could be used to cut out the Torr-seal if a piece needed to be replaced. Earlier iterations of the reaction box without this feature required a complete rebuild if something broke. There was a small amount of space near each of the alumina tube ports where the gas was not reacted-these areas can be seen in the right image in Figure 3.9. These areas are small in comparison to the reaction zone and were neglected in the experimental analysis. The two rectangular quartz pieces were for optical measurements and were polished to optical grade clarity before being 
mounted. This permitted optical emission spectroscopy measurements through the quartz sides. The polishing was done by hand using incrementally finer grit paper down to as small as 0.1 micron grit size. Note, each piece of the middle section was either ceramic or glass to provide insulation and to avoid surface discharges.

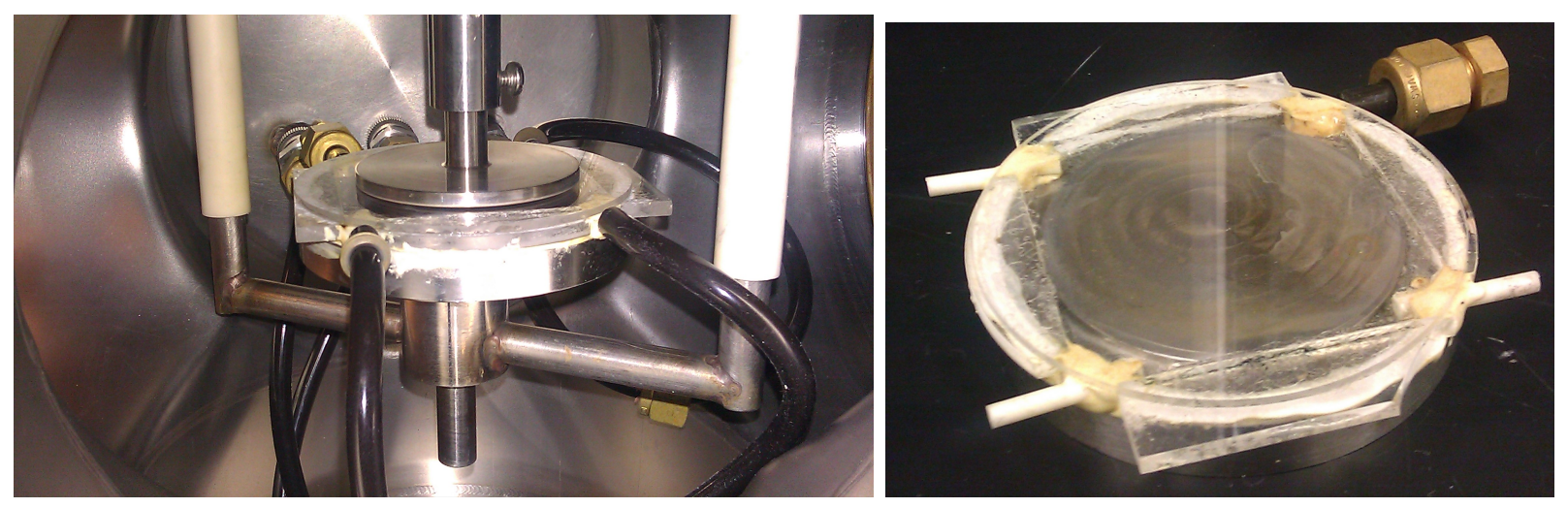

Figure 3.9: Left, the reaction box installed in the reaction chamber. Right, a completed reaction box

The top layer of the reaction box was a 3" diameter 1/16" thick quartz disk, which acted as the DBD dielectric. To mount the disk, the middle layer was Torr-sealed into place as flat as possible. Then, any Torr-seal that protruded from the surface was shaved smooth, giving a smooth surface for adherence of the quartz disk. Once smooth, the quartz disk was sealed to the top surface using Crystalbond, an epoxy that provides a strong vacuum seal and is easily removed with heating. The easily removable seal allowed for the addition of catalysts and cleaning of the reaction box. It was found that the crystal bond strength was modest and the system could not handle large pressure differentials. Therefore, it was important during setup and runs to make sure the pressure difference between the inside and outside of the reaction box was never more than $\sim 200$ Torr.

Once the middle layer was in place and polished smooth, the easiest way to mount the quartz disk was to dissolve the Crystalbond into a solvent and spread it on both the disk 
and middle layer surfaces, as thick as possible. The surfaces were allowed to dry such that the Crystalbond solution became pasty. Once pasty, the top quartz piece was placed onto the smoothed surface of the middle piece, making sure it sealed around the edges. The seal was monitored by looking at the air bubbles forming between the two surfaces; elimination of the bubbles ensured a strong seal. Once the pieces were together and sealed, pressure was applied to the top of the assembly to maintain a proper seal during curing. It took several hours for the Crystalbond to harden and the solvent to evaporate. Heating the system in an oven or other heating system greatly sped up the curing process. If the solvent wasn't allowed enough time to evaporate and the system was put under pressure or vacuum, the solvent would bubble, which ruined the bond and often covered various surfaces in amorphous Crystalbond.

If the quartz/Torr-seal surfaces were not smooth enough, another method was used to apply the Crystalbond solution. The Crystalbond, in solvent, was applied and allowed to become pasty. Ground up Crystalbond, with a sand-like consistency, was pushed into both of the pasty surfaces creating a thin surface of solid Crystalbond on the amorphous Crystalbond. Then the two pieces were joined together as before and placed in an oven at the melting temperature of the Crystalbond with a heavy weight to maintain compression. Once all of the Crystalbond became amorphous, the oven was turned off. The surfaces sometimes shifted off center during heating and had to be slid back in place while the Crystalbond was still amorphous, after which the reaction box was allowed to slowly cool with the heavy weight still on it. This method created a slightly thicker and much stronger bond than the other method. Note, if the top layer slid too much and Crystalbond leaked into the reaction area, the process was restarted. 


\subsubsection{Catalyst Mounting}

The catalyst used in VADER was commercial P25 $\mathrm{TiO}_{2}$. The quartz disk comprising the top layer of the reaction box had a thin film, $<0.25 \mathrm{~mm}$ thick, of the $\mathrm{TiO}_{2}$ catalyst applied in an $\sim 2$ inch diameter patch at the center of the disk-see Figure 3.10. The mounting process was done by Christopher Matranga of NETL by hydrolizing the $\mathrm{TiO}_{2}$ in liquid titanium isopropoxide, which was then applied to the quartz surface and allowed to dry. This created an amorphous $\mathrm{TiO}_{2}$ film with the P25 particles embedded in the film. The film was not seen to change after long periods of time within the plasma, but was found to easily rub off. Therefore, special attention was paid when mounting the quartz disk. Only a single disk with catalyst was used for all of these experiments.

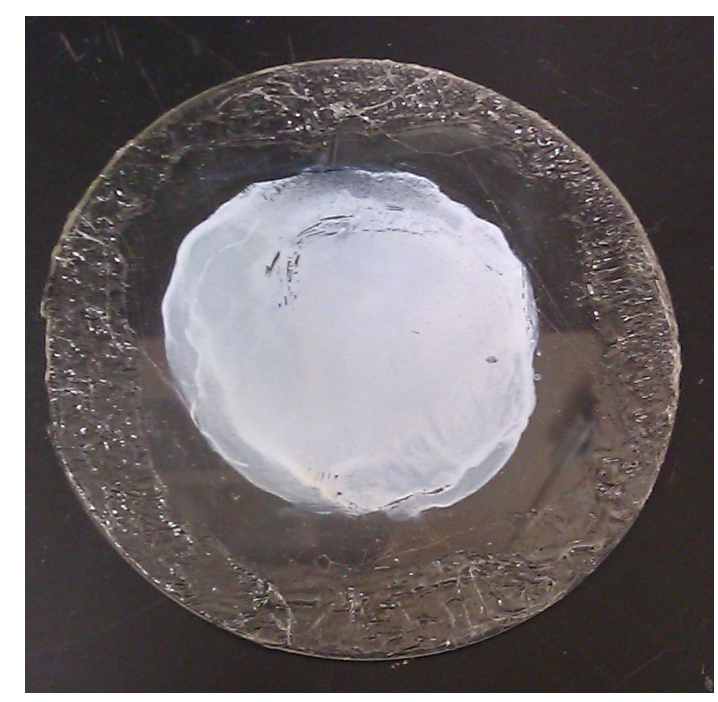

Figure 3.10: The quartz disk with the $\mathrm{TiO}_{2}$ film catalyst applied to its surface. This disk was used in all of the catalyst experiments. The edges look rough because they are covered in Crystalbond 


\subsubsection{Gas System}

The VADER gas handling system, seen in Figure 3.11, was designed to allow a known volume and mixture of gas at a specific temperature to flow into the reaction chamber. The reacted gas composition was measured using a residual gas analyzer. The input side of the gas system was set up such that a pair of gas tanks, carbon dioxide and argon from AIRGAS, could be attached to a set of MKS 100 and 500 SCCM mass flow controllers, which then piped the combined gas into a heating coil. Before each experimental run the entire gas system was flushed out.

A heating coil was used bring the gas to the desired operating temperature. The heating coil was 12' of 1/4" SS tube (copper was attempted but was found to quickly oxidize at the temperatures needed) wrapped into a $21 / 4$ " diameter tightly packed coil then wrapped in heating tape with a large amount of insulation around the heating tape. At the center of the SS tube a piece of quartz glass was inserted to insulate the inside of the coil, as can be seen in Figure 3.12. The long path length and heavy insulation were important since the thermal conductivity of gases was very low and the long path length gave plenty of time for gas mixing before reaching the reaction box. To ensure the temperature of the gas leaving the heating coil was still at the correct temperature, heavy insulation was installed along the entire length of pipe leading up to the reaction chamber. A thermocouple was installed approximately 3" down the line from the heating coil to control the heater's feedback loop. The feedback loop was controlled by an Omega programmable temperature controller set to a PID (proportional, integral, derivative) loop. After testing and retesting the heating system,

it was eventually determined it was not possible to prevent the gas from cooling down well before reaching the reaction chamber. Therefore, variations in the initial temperature were disregarded in the final experimental analysis. 


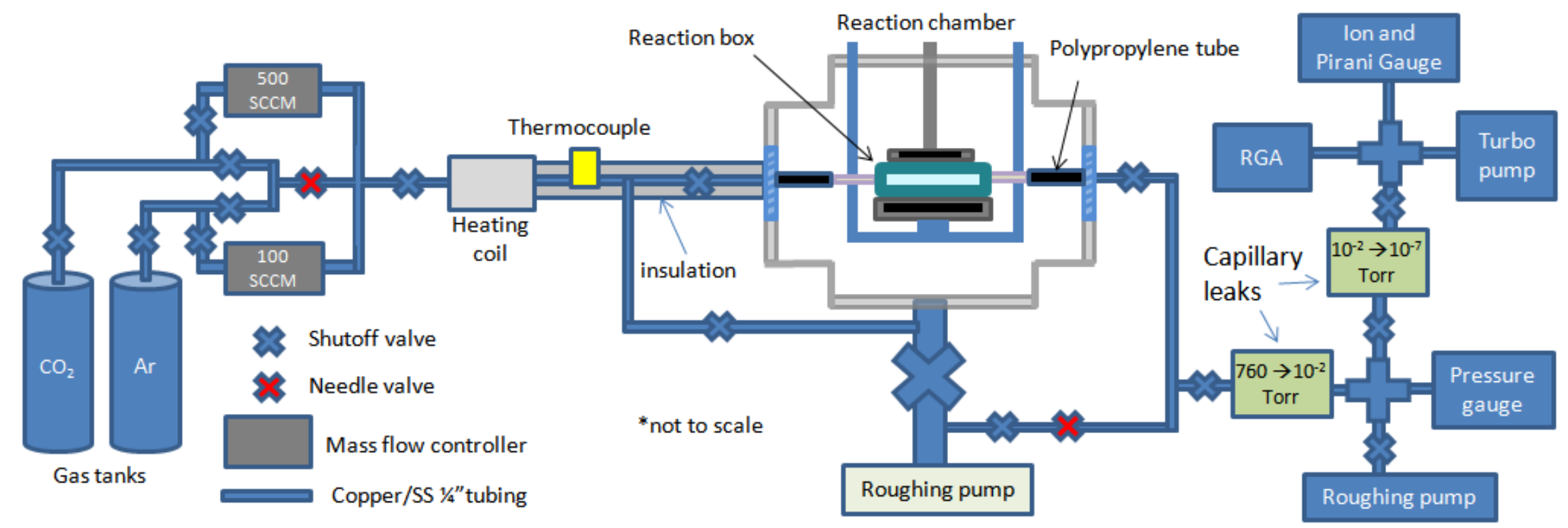

Figure 3.11: A diagram of the VADER Gas system 
After the gas left the heating coil, it entered a Swagelok $\mathrm{T}$ junction and went in one of two directions, either into the reaction chamber or the reaction box. In the first case, the gas followed a 1/4" copper tubing to a shutoff valve which lead to the bottom flange of the chamber and directly into the reaction chamber. In the second case, the gas entered a feedthrough flange on the backside of the reaction chamber, which consisted of an 8 " blank flange with three 1/4" Cajon feedthroughs welded onto it for a pressure gauge, a gas inlet and a gas exhaust. The gas entered the back side flange of the reaction chamber through a 1/4" copper tube fed through the gas inlet Cajon fitting, the copper tube then connected to a 1/4" polypropylene tube using a 1/4" Cajon to $1 / 4$ " Swagelok fitting. The polypropylene tube led to the gas inlet of the reaction box or was left open to the chamber. The pressure gauge, an Ashcroft compound vacuum pressure gauge, was connected to the end of a 1/4" SS tube bent into an L, which fed into the Cajon feedthrough. Just like the gas feed, a polypropylene tube was connected to the SS tube to connect to the port on the reaction box for a pressure gauge. For the reaction box exhaust, another polypropylene tube was attached to the exhaust port and connected to another 1/4" Cajon to 1/4" Swagelok fitting and a $1 / 4$ " copper tube. From there, the $1 / 4$ " copper tube exited the system through the exhaust Cajon fitting and split into a throttled roughing pump (the exhaust system) and the capillary leak apertures for the atmospheric pressure RGA (Discussed in Section 3.2.1). The exhaust system was a Pfeiffer Xtra dry vacuum pump attached to a needle valve. The needle valve was used to throttle the gas flow and control the pressure within the reaction box. For experimental runs, the valve was slowly tweaked until the pressure within the reaction chamber was constant at 760 Torr, at which point the volume of gas entering and exiting the reaction box was considered constant. Once this setting was found, the pressure stayed constant for multiple hours. 


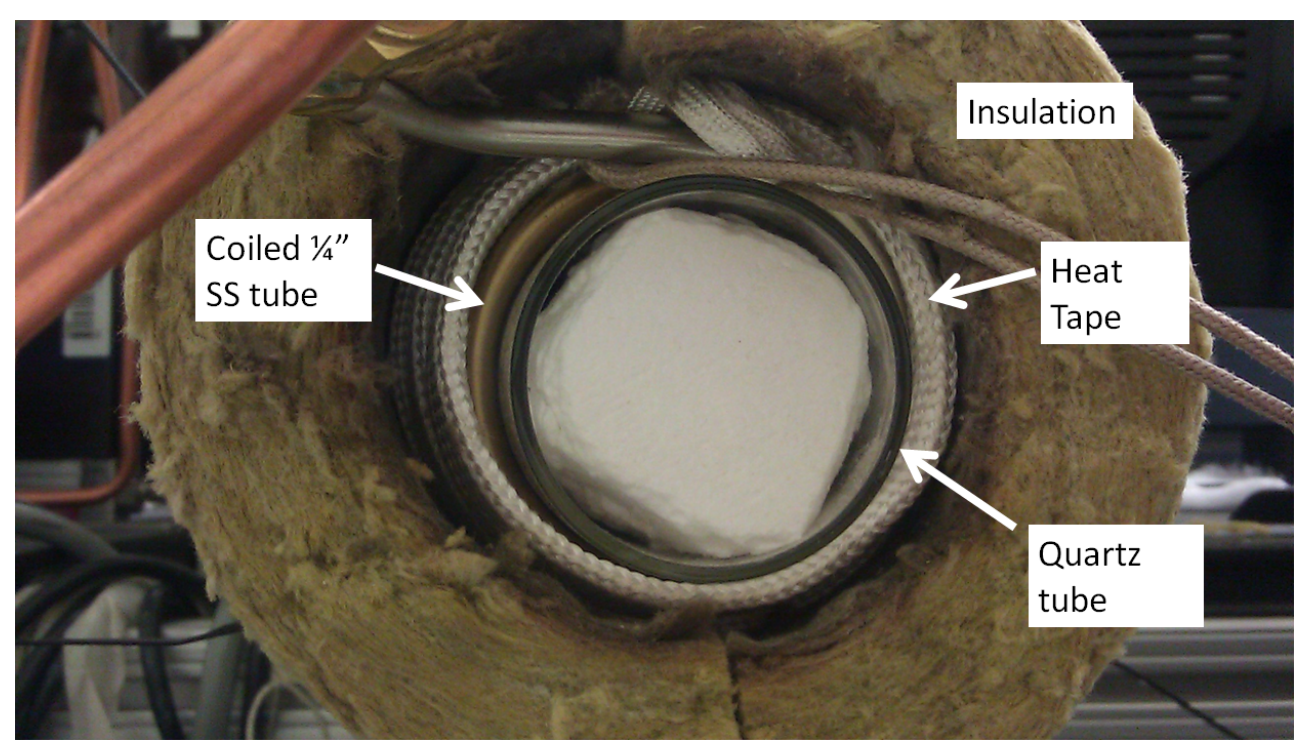

Figure 3.12: A picture of the VADER heating coil

The bottom flange of the reaction chamber was a modified 8" blank CF flange with a single KF-40 connector welded to the center of the flange. The KF-40 connected to a 2-3/4" piping with a 1/4" Swagelok fitting and another KF-40 connector. The Swagelok fitting teed off of the 1/4" copper piping running from the gas inlet and allowed for separate gas fills of the reaction chamber and the reaction box. The KF-40 connector ran to a large shutoff valve, on the other side of which was the same roughing pump used for the gas exhaust. The roughing pump was used to control the pressure differential between the reaction box and the reaction chamber. The bottom flange setup is the bottom portion of the reaction chamber shown in Figure 3.11.

\subsection{Diagnostics}

Determination of the efficiency and plasma characteristics of VADER required measurements of the gas composition, the system input power, and the emission spectrum. The gas com- 
position was measured using a real-time residual gas analyzer (RGA) and the power was determined through a number of current and voltage measurements. Due to the high voltages needed for a DBD and the problems this caused with in-situ probes, plasma diagnostics were limited to optical systems. Thus, VADER was built with considerable optical access, three optical glass ports on the reaction chamber and optical grade polishing done to the quartz pieces on the reaction box.

\subsubsection{RGA}

The RGA was used to find the chemical composition of the input and exhaust. Since a RGA only works at pressures of $10^{-4}$ Torr or less a specially calibrated capillary leak system designed by Vacuum Technology Incorporated (VTI) was used to reduce the atmospheric pressure gas source down to the RGA pressure range. VTI uses a pressure step-down process with two capillary calibrated leaks. A capillary leak is a specially crimped pipe that allows a very small and exact amount of gas flow through based on a pressure differential between the input and output. The first capillary leak connected directly to the exhaust line, before the needle valve. Behind the first capillary leak was a Varian SH-110 roughing pump which brought the pressure down to $10^{-2}$ torr from 760 Torr. This pressure was monitored with a Lesker convectron gauge. At that point, the second capillary leak, connected to a Pfeiffer TSH-521 turbo pump, brought the pressure down to $10^{-8}$ Torr. In the same cavity as the turbo pump was an SRS RGA100, an ion pressure gauge, and a convectron pressure gauge. The setup is shown in Figure 3.11.

The sampling system allowed for a very small amount of gas from the reaction chamber to be sampled continuously by the RGA. Since the gas needed to travel a long distance and through many small openings, there was a $\sim 30$ second lag time between the time when the 
plasma was first created and the time the processed gas first reached the RGA. Thus, plasma runs had to be at least 5 minutes for accurate RGA measurements (as shown in Figure 3.13).

Some inherent issues with RGAs also have to be considered. For instance, an RGA specifically measures the $\mathrm{E} / \mathrm{m}$ (charge over mass) of the different input gases. Since some of the components of a gas mixture have the same $\mathrm{E} / \mathrm{m}$, it can sometimes be impossible to distinguish between two such gases in an RGA. One example is $\mathrm{CO}$ and $\mathrm{N}_{2}$, both have an atomic weight of 28 and are assumed to be singly ionized in the RGA. Another issue with RGAs is an error of at least $10 \%$ in the relative pressure measurements. The error manifests as a slow drift in gas pressures over time, despite no changes in the system parameters. This error made it difficult to measure changes in the $\mathrm{CO}_{2}$ pressure between when the plasma was on and off, unless at least several percent of the $\mathrm{CO}_{2}$ was reacted. Therefore, the conversion calculations were done using calibrated pressure measurements of the product gases (CO and $\mathrm{O}_{2}$ ). $\mathrm{CO} / \mathrm{N}_{2}$ and $\mathrm{O}_{2}$ pressures were very small when the plasma was off, thus any change in their relative pressures had better signal to noise than $\mathrm{CO}_{2}$ measurements. To avoid further error due to the slow pressure drift, only the instantaneous changes in gas pressures were measured and runs were repeated if the drift was greater than $10 \%$. It should be noted that a small air leak consisting of less than a percent of the gas was found in the system. The leak was considered to be too small to affect the chemistry and did not change significantly over time, thus it did not affect any of the measurements.

The most important step in using a RGA is calibration. We pumped a known composition of gas into the system at different pressures and read the corresponding RGA pressures. The RGA pressures along with the system pressure values were then recorded and used to create a calibration curve. Due to the slow drift, this was done multiple times and averaged to obtain a final calibration. The calibration was used to convert RGA values to the actual 


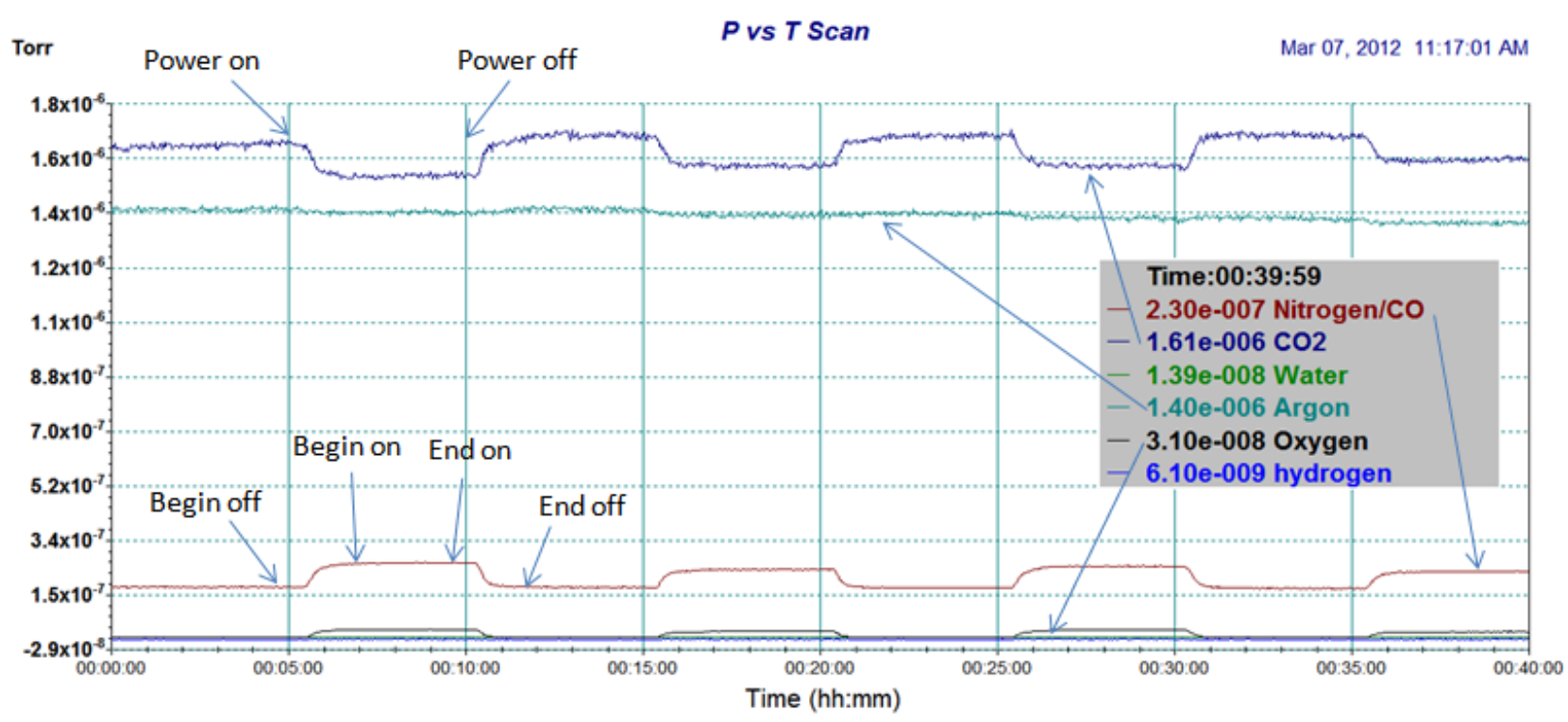

Figure 3.13: An example of an RGA readout - pressure versus time. "Power on" denotes when the high voltage power supply was turned on and "Power off" was when it was shutoff. Note the 30 second lag time between the power being cycled and the change in pressure values. "Begin off" denotes the approximate time when a pressure reading was taken before the plasma was turned on. "Begin on" is the reading after the plasma was turned on. "End on" is before the plasma was turned off and "End off" is after the plasma was turned off. The argon line is a good example of the slow pressure changes seen in RGAs. These data are from readings taken on 3/7/2012 using the DIRIV10 power supply with a 200 SCCM flow, $60 \% \mathrm{CO}_{2} 40 \% \mathrm{Ar}$ and the $\mathrm{TiO}_{2}$ catalyst.

pressures in the reaction chamber and ultimately calculate the number of $\mathrm{CO}_{2}$ molecules converted in the plasma. The calibration gases used were Ar, CO and air. All gases, other than air came from Airgas, and were at least 99\% pure. Air from the room was used to calibrate the system for $\mathrm{O}_{2}$ and was considered to be $78 \% \mathrm{~N}_{2}$ and $21 \% \mathrm{O}_{2}$.

\subsubsection{Optics}

The three optical glass viewports on the reaction chamber were comprised of two 8" CF Kodial glass viewports (Lesker: VPZL-800) and an 8" fast entry door with kodial glass (Lesker: DS-LL0800VP). These each allowed optical access in the near-UV, visible and near- 
infrared (300-2500nm). Optical diagnostics were accomplished using a Matisse-DR ring dye Laser (details of which are in Ref. [102]), and a model number 63355 Oriel calibration lamp. The light from each source was coupled into VADER using optical fibers which led to a set of injection optics.

All of the optics were mounted on two optical breadboards mounted on the VADER system stand, each on opposite sides of the reaction chamber. The injection optics consisted of a set of $2.54 \mathrm{~cm}$ diameter optics from Thor labs. The optical fiber from the light sources connected to a lens tube holding an optical fiber coupler. The light from the coupler, between $300-1100 \mathrm{~nm}$, was collimated using a N-BK7 $10 \mathrm{~cm}$ focal length plano-convex lens. The collimated light then fell on a N-BK7 $30 \mathrm{~cm}$ focal length plano-convex lens which focused the light into the plasma, as shown in Figure 3.14. The lens tubes were held in place and the angles were adjusted using a Thor labs kinematic mount. The height of the optics was adjusted using optical posts in post holders. The collection optics are the same as the injection optics, except $5 \mathrm{~cm}$ diameter lenses were used instead of the $2.54 \mathrm{~cm}$ diameter lenses.

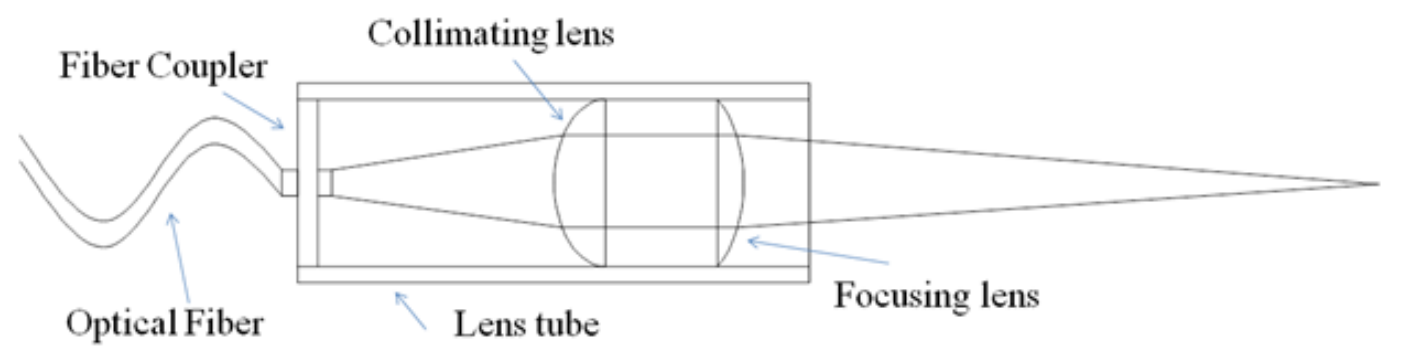

Figure 3.14: The collection and injection optic configurations used on VADER

The light from the collection optics was coupled into two spectrometers, an Ocean Optics HR4000 and a McPherson 209 1.33m Scanning Monochromator. The HR4000 has a wavelength range of $200-1100 \mathrm{~nm}$ with a resolution of $\sim 0.25 \mathrm{~nm}$. The $1.33 \mathrm{~m}$ scanning 
monochromator has a wavelength range of $\sim 4 \mathrm{~nm}$, the center of which may be scanned between $\sim 185$ and $1300 \mathrm{~nm}$ with a resolution of $0.01 \mathrm{~nm}$. The HR4000 was calibrated by Ocean Optics and the scanning monochromator was calibrated using an Oriel calibration lamp.

\subsubsection{Power Measurements}

To find the forward power (the energy entering the DBD) the voltage and current from the two power sources were measured. The Trek 10/10B power supply had built in BNC jacks for voltage and current measurements. The DIDRIV10 power supply had no such connectors, therefore a Rogowski coil and a voltage divider were used to find the current and voltage, respectively. The Rogowski coil was a 1V/1A Pearson current monitor and the readings were performed on the power supply return line. Since high voltage and high frequency sources were used, a custom voltage divider was needed. The voltage was divided through two 10 $\mathrm{M} \Omega, 12.5 \mathrm{~W}$ resistors in series followed by a $10 \mathrm{k} \Omega$ resistor to ground, the voltage was read between the $10 \mathrm{k} \Omega$ resistor and ground for a voltage division of $\sim 2000$. To protect personnel from the HV, the voltage divider was built inside a 12 " $\times 7 " \times 4$ " grounded aluminum project box; each cable was rated for $50 \mathrm{kV}$, and all of the lines were fed through PVC pipes. Any breaks in the line were kept far away from conductive materials. A picture of the voltage divider is shown in Figure 3.15. Two of the three holes in the voltage divider were for the HV line feed through and the third connected the divided signal to a BNC, which was monitored with an oscilliscope.

When the DIDRIV10 power supply was attached to the voltage divider at higher powers and there was not a path to ground (the DBD in this case), a DBD and/or corona discharge formed across the length of the HV lines. However, the voltage divider resistors were able 


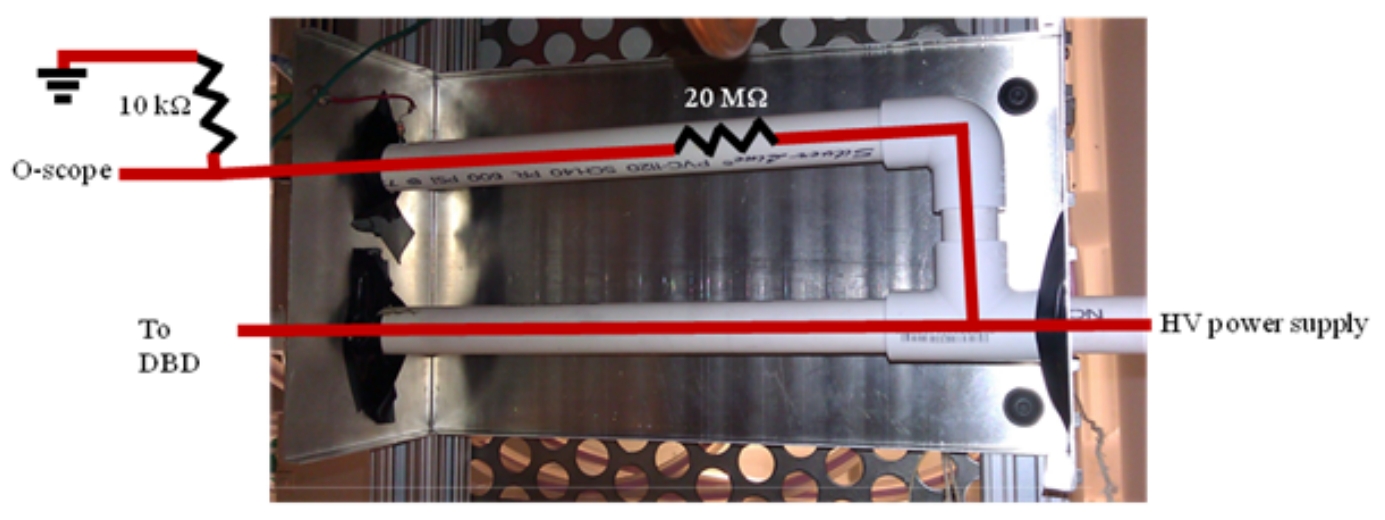

Figure 3.15: The voltage divider added between the DIDRIV10 power supply and the DBD electrodes to find the electrode voltages. It divided the voltage by a factor of 2000 .

to handle the entire load of the DIDRIV10 power supply.

To calculate the forward power, the voltage (V) and current (I) measurements were averaged over 100 waveforms and then multiplied together. Reflected power (power reflected back into the power supply) was removed from the measurements by setting negative values of $\mathrm{I} \times \mathrm{V}$ to zero. The root mean square of this final waveform gave the average forward power of the system. The voltage divider was designed to have a maximum leakage rate of $<1 \%$ of the total power and was considered negligible. 


\section{Chapter 4}

\section{Optical Diagnostics in VADER}

Plasma diagnostics are key to understanding a plasma. Immersion probes in a DBD are not a viable option for plasma diagnosis due to the high voltages, which would damage such probes, and the high pressures at which many immersive diagnostics cease to work. Thus, optical diagnostics were the main diagnostic tool used in VADER.

Several optical diagnostic techniques were attempted in VADER, including molecular spectroscopy, Stark broadening spectroscopy, line ratio spectroscopy, laser induced fluorescence and absorption spectroscopy. However, results were only obtained using the molecular spectroscopy and Stark broadening optical emission spectroscopy (OES) techniques. OES is passive spectroscopy based on the optical range of electromagnetic radiation, which occurs due to particles or molecules relaxing from a higher energy state to a lower energy state. From the emission spectrum of a plasma, it is possible to determine the electron density $\left(N_{e}\right)$ and electron temperature $\left(T_{e}\right)$, if a sufficiently detailed theoretical model is available. Unlike other optical methods, the experimental setup for OES is very simple, requiring only

a spectrometer, a method of getting the light from the plasma into the spectrometer, and a calibration lamp. The apparatus used with VADER is described in section 3.2.2. How- 
ever, the theoretical models are often quite complicated, especially for atmospheric pressure plasmas which often require a collisional radiative model.

\subsection{Molecular Band Spectroscopy}

The first optical emission spectrum observed in VADER for a $\mathrm{CO}_{2}$ plasma was the molecular emission band between 300-700 nm using the Ocean Optics HR4000 spectrometer, an example of the band is shown in Figure 4.1. The lines were very dim and full detail could only be seen when the power supplies were set to their max settings (TREK power supply) using exposure times of up to 30 seconds and averaging over multiple runs.

Comparing the emission spectrum of VADER to those found by Rond et al. [39] (see Figure 4.2), revealed that these lines correspond to three different $\mathrm{CO}$ electronic bands: the Angstrom band $\left(B^{1} \Sigma^{+} \rightarrow A^{1} \Pi\right)$, the Triplet band $\left(d^{3} \Delta \rightarrow a^{3} \Pi\right)$ and the Asundi band $\left(a^{\prime 3} \Sigma^{+} \rightarrow a^{3} \Pi\right)[103]$. As there are no other molecular emission bands within this wavelength

range, the measurements demonstrate that the VADER plasma dissociates $\mathrm{CO}_{2}$ into CO. While each of the bands is detectable, certain bands dominate. The Angstrom band is the strongest, the Asundi band is dimmer and the Triplet band is even more dim. 


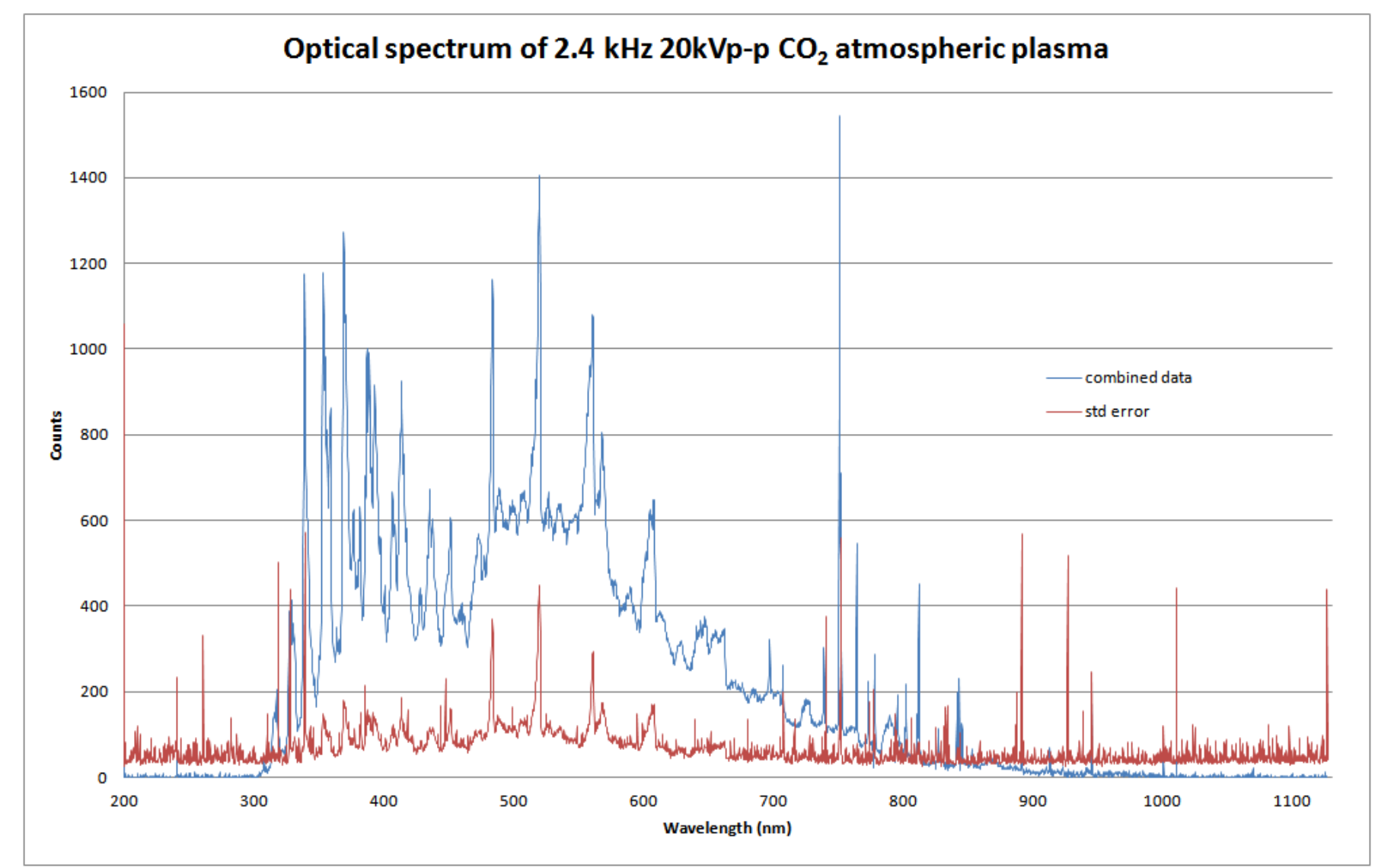

Figure 4.1: A sample emission spectrum of a pure $\mathrm{CO}_{2}$ plasma in VADER set to a voltage and frequency of $20 \mathrm{kVp}-\mathrm{p}$ and $2.4 \mathrm{kHz}$, respectively. The bulk of the emission lines from $300-700 \mathrm{~nm}$ are due to transitions between the rotational bands of CO. 


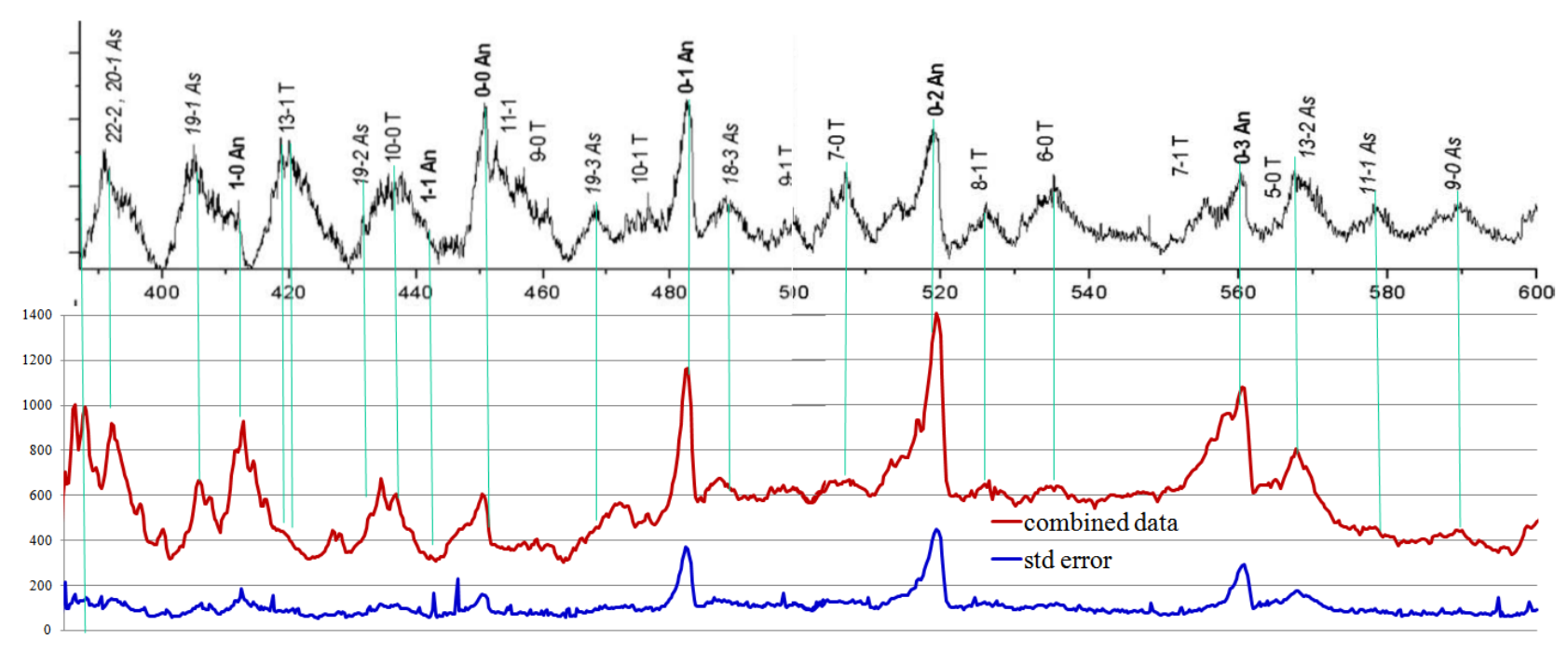

Figure 4.2: $\mathrm{An}=$ Angstrom band, $\mathrm{As}=$ Asundi band, $\mathrm{T}=$ Triplet band, $\mathrm{A}-\mathrm{B}, \mathrm{A}$ stands for the upper vibrational state and B the lower vibrational state. The top graph is the CO spectrum found by C. Rond et al. [39] and the bottom graph is the VADER spectrum. The $\mathrm{CO}$ angstrom band dominates the VADER spectrum, the Asundi band is weakly excited and the Triplet band is barely evident.

Analysis of band spectroscopy measurements usually entails matching an experimental emission spectrum to a mathematical model of the emission spectrum to find the electronic, rotational and vibrational energies of the plasma. At atmospheric pressures, this requires a clean emission band and a comprehensive collisional radiative model built for atmospheric pressures. Collisional radiative models require knowledge of the collisional and spontaneous transitions from all energy states of each atom of interest. Since all of these transitions are often not readily available, this makes the development of collisional radiative models complicated [104]. The analysis is further complicated by the overlapping CO molecular bands, thus making such analysis well beyond the scope of this work. However, there are several groups and commercial codes that are developing collisional radiative models to look into $\mathrm{CO}_{2}$ processes. For example, the SPECAIR and PASTIS codes along with several space plasma codes already exist $[105,106]$. Note, the spectrum shown was not absolutely 
calibrated, therefore detailed analysis should not be applied to this spectrum.

\subsection{Stark Broadening Spectroscopy}

The unperturbed spectral lines of atoms, ions and molecules are usually very narrow, but due to various broadening mechanisms, emission lines are often widened and slightly shifted away from their natural wavelength. By deconvolving a measured line shape in terms of the various broadening mechanisms, it is possible to determine the electron density (Stark Broadening), local electric and magnetic fields (Stark and Zeeman effect, respectively), neutral/ion temperatures and the bulk velocity of neutrals/ions in a plasma (Doppler broaden-

ing) [107-109]. Many papers on line broadening have been compiled by the NIST atomic spectral line broadening bibliographic database [110].

Stark broadening spectroscopy involves developing a complete model of an isolated spectral line, taking into account all forms of line broadening, and extracting the Stark broadened components to determine the electron density, $n_{e}$. For VADER, we modeled the $794.8176 \mathrm{~nm}$ neutral argon spectral line. The relevant broadening mechanisms include natural broadening, Doppler broadening, instrumental broadening, the Zeeman Effect, and pressure broadening. Pressure broadening consists of Van Der Waals broadening, Resonance broadening, Stark broadening and ion Stark broadening. Some other concerns when using OES methods are self-absorption by the background gas and integration over the line of sight of the optics.

\section{Broadening Mechanisms}

Natural broadening is due to the Heisenberg uncertainty principle when applied to the lifetime of an excited state and results in an unshifted Lorentzian emission profile [111]. The 
shorter the decay time, the more uncertainty there is in the energy of the decay and the larger the broadening [112]. In the optical/UV range natural broadening is very small, often less than $.0001 \mathrm{~nm}$, making it negligible in comparison to other broadening mechanisms and ignorable for broadening calculations of emission lines in atmospheric pressure plasmas [113].

Doppler (thermal) broadening is due to the emitting particle's velocity and is based on the Doppler effect. Assuming the emitting particles are in local thermodynamic equilibrium (LTE), a Doppler broadened spectral line has a Gaussian distribution with a half-width half-maximum $\left(\mathrm{HWHM}, w_{D}\right)$ of $[114]$

$$
w_{D}(\mathrm{~cm})=7.162 \times 10^{-7} \lambda_{0} \sqrt{T_{g} / M}
$$

where $\lambda_{0}$ is the unshifted wavelength of the spectral line $(\mathrm{cm}), T_{g}$ is the gas temperature (in $\mathrm{K}$ ) and $M$ is the emitting particle's mass (in amu). If the bulk of a plasma has nonzero flow, Doppler broadening causes a shift in the central wavelength of the spectral line (which depends on the direction of flow). Since emitters are usually heavy particles (ions or neutrals), relativistic effects can often be ignored (except in very hot plasmas), making the frequency shift of the spectral line $(\delta v)$

$$
\frac{\delta v}{v_{0}} \approx \frac{v_{s}}{c}
$$

where $v_{0}$ is the unshifted frequency of the spectral line, $v_{s}$ is the bulk velocity of the emitting particles and $c$ is the speed of light. Doppler spectroscopy uses the shifts in the central wavelength due to particle velocities to determine ion/neutral velocities (a common practice in astrophysics to determine the velocity of of interstellar bodies) [108]. However, ion and neutral velocities and gas temperatures in a DBD are very small $(<0.05 \mathrm{eV})$, meaning Doppler 
broadening and Doppler shifts are ignorable in VADER.

Instrumental broadening occurs in all experimental detection systems. This broadening can have multiple sources, some examples are too large of a spectrometer slit size, diffraction grating imperfections or blooming in a CCD array. Since the sources of instrumental broadening can be highly non-uniform, the instrumentally broadened spectral line may not follow any typical pattern or distribution. The instrumental broadening profile may be found by sweeping the spectrometer over a line with an intrinsic width small in comparison to the spectrometer's detection width. The deconvolution process must then include it as an additional broadening source, which increases error and increases the calculation difficulty. However, Konjevic mentions that this broadening can practically be eliminated by using a spectrometer with a resolution that is one-tenth or less than the observed spectral line (this was accomplished using the McPherson 209 scanning monochromator) [113]. However, in the case of VADER, it is presumed that it applies a Gaussian broadening of the lines due to imperfections in the diffraction grating and the age of the spectrometer.

The Zeeman effect arises when a magnetic field is applied to a bound electron and causes the spectral lines of a transition to split into multiple closely spaced lines. This effect arises from the interaction between the orbital angular momentum of the emitting particle $(\vec{L})$ and the electron orbit magnetic moment $(\vec{S})$ with the external field. The energy split of the emitting particles $(\Delta E)$ is

$$
\Delta E=\frac{e}{2 m_{e}}(\vec{L}+2 \vec{S}) \bullet \vec{B}=g_{L} \mu_{B} m_{j} B
$$

where $e$ is the charge of an electron, $m_{e}$ is the electron mass, $\vec{B}$ is the magnetic field vector, $g_{L}$ is the Landé g-factor, $\mu_{B}$ is the Bohr magneton $\left(\frac{e \hbar}{2 m_{e}}\right), m_{j}$ is one of the total orbital 
angular momentum states of the particle (total angular momentum is $\vec{J}=\vec{L}+\vec{S}$ ) and B is magnetic field amplitude. $\Delta E$ is very small except when there is a large magnetic field [108]. Since no magnetic fields are used in a DBD, the Zeeman effect was ignored.

Van der Waals broadening arises from the perturbation of the emitting particle by Van der Waals forces (dipole-dipole interactions). The Van der Waals forces apply a shifted Lorentzian profile to the spectral line shape. Hans Griem originally derived the HWHM and shift in the Van der Waals profile $\left(w_{V D W}\right)$, which was simplified by Konjevic to [113-115]

$$
w_{V D W}(\mathrm{~cm})=8.18 \times 10^{-12} \lambda_{0}^{2} N_{p}\left(\bar{\alpha} \bar{R}^{2}\right)^{2 / 5}\left(\frac{T_{g}}{\mu_{P E}}\right)^{3 / 10}
$$

where $\lambda_{0}$ and $T_{g}$ have units of $\mathrm{cm}$ and $\mathrm{K}$, respectively. $N_{p}$ is the perturber density (usually the neutral density, $\mathrm{cm}^{-3}$ ), $\mu_{P E}$ is the reduced mass of the perturber and emitter (amu), and $\bar{\alpha}$ is the polarizability of the perturber $\left(\mathrm{cm}^{3}\right) \cdot \bar{\alpha}$ for many different elements was tabulated by Cox [116]. For elements not tabulated, $\bar{\alpha}$ can be estimated using [115]

$$
\bar{\alpha}=\frac{9}{2} a_{0}^{3}\left(\frac{3 E_{H}}{4 E_{2 P}}\right)^{2}
$$

where $a_{0}$ is the Bohr radius $\left(\sim 5.29 \times 10^{-9} \mathrm{~cm}\right), E_{H}$ is the ionization potential of hydrogen $(13.6 \mathrm{eV})$ and $E_{2 P}$ is the energy of the first excited level of the perturber atom. $\bar{R}^{2}$ is the difference in the squares of the coordinate vectors of the upper $\left(\bar{R}_{U}^{2}\right)$ and lower $\left(\bar{R}_{L}^{2}\right)$ energy levels of the emitter (unitless).

$$
\bar{R}^{2}=\bar{R}_{U}^{2}-\overline{R_{L}^{2}}
$$


Using the Coulomb approximation, $\overline{R_{U}^{2}}$ and $\overline{R_{L}^{2}}$ is calculated using

$$
\bar{R}_{j}^{2}=\frac{n_{j}^{* 2}}{2}\left[5 z^{2} n_{j}^{* 2}+1-3 l_{j}\left(l_{j}+1\right)\right]
$$

where $l_{j}$ is the orbital quantum number, $z$ is the number of effective charges (usually 1 ) and $n_{j}^{* 2}$ is the effective quantum number.

$$
n_{j}^{* 2}=\left(\frac{E_{H}}{E_{\text {Ion }}-E_{j}}\right)^{1 / 2}
$$

Where $E_{\text {Ion }}$ is the ionization energy of the emitting particle and $E_{j}$ is the upper or lower energy level of the emitter for the observed transitions. The shift in the Van der Waals profile $\left(d_{V D W}\right)$ was estimated by Hans Griem to be [115]

$$
d_{V D W} \approx-\frac{2}{3} w_{V D W}
$$

The above equations are only valid if the impact approximation is valid, the adiabatic approximation is valid and the perturber and radiator are compact, such that Van der Waals broadening is dominated by strong collisions. These limitations are further described by Griem [115] and are all valid within VADER. Due to the high neutral densities in atmospheric plasmas, Van der Waals broadening is almost always a significant effect on the spectral line width. Therefore, it could not be ignored during the VADER experiments.

Resonance broadening is due to energy exchange between like perturbing and emitting particles. This form of broadening only occurs at significant levels when there is a resonant dipole transition from either the upper or lower energy state of the emitting particle to the ground state [117]. The distribution for resonance broadened spectral line is Lorentzian and 
has a negligible shift in the central wavelength [115]. The HWHM of a resonance broadened line shape $\left(w_{\text {Res }}\right)$ follows

$$
w_{\text {Res }}=8.61 \times 10^{-14}\left(\frac{g_{i}}{g_{k}}\right)^{1 / 2} \lambda_{0}^{2} \lambda_{R} f_{R} N_{g}
$$

where $g_{i}$ and $g_{k}$ are the statistical weights of the of the upper and lower state, $\lambda_{R}$ and $f_{R}$ are the wavelength and absorption oscillator strength (or f-value) of the resonant transition, respectively, and $N_{g}$ is the density of ground state particles. The $794.8176 \mathrm{~nm}$ neutral argon line used in these experiments did not have a valid resonant ground state dipole transition. Therefore, resonance broadening was ignored in VADER.

Stark broadening and Ion Stark broadening are due to the combination of electron and ion impact with the emitting particle, respectively, as well as the local micro-electric fields created by the electron and ion populations, respectively. The electron collisions and microelectric fields create a shifted symmetric Lorentzian. The ions create a shifted asymmetric Lorentzian; the asymmetry is due to the quadratic Stark effect [113]. The theoretical HWHM $\left(w_{S t}\right)$ and shift $\left(d_{S t}\right)$ for neutral atom Stark broadening is calculated using

$$
\begin{gathered}
w_{S t}\left(N_{e}, T_{e}\right) \cong w_{e}\left(T_{e}\right) N_{e}\left[1+1.75 \times 10^{-4} N_{e}^{1 / 4} \alpha\left(T_{e}\right)\left(1-0.068 N_{e}^{1 / 6} T_{e}^{-1 / 2}\right)\right] \times 10^{16} \\
d_{S t}\left(N_{e}, T_{e}\right) \cong d_{e}\left(T_{e}\right) N_{e}\left[1+2.0 \times 10^{-4} N_{e}^{1 / 4} \alpha\left(T_{e}\right)\left(1-0.068 N_{e}^{1 / 6} T_{e}^{-1 / 2}\right)\right] \times 0.5 \times 10^{16}
\end{gathered}
$$

where $w_{e}$ and $d_{e}$ are the electron impact half-width (HWHM) and shift, respectively, and $\alpha$ is the ion broadening parameter, as given in [118] at $N_{e}=10^{16} \mathrm{~cm}^{-3}$ [113]. For singly ionized atoms, $\alpha$ is zero, simplifying the equation significantly. The above equations are only valid as long as the effects of Debye shielding have a minimal effect on broadening and there is a 
minimal amount of Stark splitting of the lines [113].

Self-absorption occurs when there is a large number of particles, usually of the same species as the emitter, at the lower energy level of the emitting transition. The particles in these states resonantly absorb the emission and proceed to either re-emit at the same wavelength or relax through a different transition pathway, reducing the emission amplitude at the original wavelength. But, because the absorption is resonant and the emitted wavelengths are broadened, absorption is proportional to the emission profile without selfabsorption. Therefore, self-broadening preferentially reduces the emission intensity at the most prominent wavelengths and effectively flattens the line profile. Self-absorption most commonly occurs when the transition goes to the ground state, due to the high density of particles at ground state energies even at relatively high temperatures. Therefore, it is usually not an issue if a non-ground state transition is used. However, self-absorption can also occur at non-ground state levels if a plasma is at a relatively high temperature or the lower level transition is a meta-stable state (the energy level has no decay pathways). Konjevic describes multiple methods to determine if self-absorption is important for a line profile [113]. For VADER, the fact that broadband absorption spectroscopy showed no absorption of any lines was used to determine that there was no self-absorption in VADER, as any sign of absorption would indicate self-absorption by the plasma.

Another complication during Stark broadening experiments occurs when the density and temperature of a plasma change significantly along the line of sight of the optics. If the different plasma densities and temperatures have significant length it causes a superposition of emission spectra. It is best to take measurements where this is not an issue, however it is often unavoidable. The solution often requires analytical reconstruction of the profile from an array of line integrated measurements, the most common methods include Abel 
inversion and tomography $[104,113,119-121]$. A DBD consists of many very narrow streamers which have large gradients in temperature and density immediately outside of the discharge center. Due to the gradients and the high temperatures required for the lines used for optical emission spectroscopy, the emission from outside of the discharge center is ignorable. Thus, despite multiple streamers entering and leaving the optical line of sight during each spectral measurement, the spectra are considered to result from the superposition of spectra from multiple streamers at identical densities.

\section{Deconvolution of the Spectra}

To deconvolve the line spectra, a theoretical line shape is fitted to the experimental spectra. In VADER, the significant broadening mechanisms are Van der Waals, instrumental, Stark and ion Stark broadening (as discussed above). Instrumental broadening follows a Gaussian profile of the form

$$
f_{\text {Gauss }}(\lambda)=\frac{1}{\sigma \sqrt{2 \pi}} e^{\frac{-\left(\lambda-\lambda_{0}\right)^{2}}{2 \sigma^{2}}}
$$

where $\sigma$ is the standard deviation in the instrumental broadened profile and $\lambda$ is any wavelength. The combination of Van der Waals, Stark and ion Stark broadening follow an asymmetrical Lorentzian profile of the form [115]

$$
f_{\text {Lor }}(\lambda)=\frac{1}{\pi} \int_{0}^{\infty} \frac{W_{R}(\beta) d \beta}{1+\left[\left(\lambda-\lambda_{0}-d_{c}\right) / w_{c}-\alpha^{4 / 3} \beta^{2}\right]^{2}}
$$

where $W_{R}(\beta)$ is the microfield strength distribution, $w_{c}$ and $d_{c}$ are the combined electron impact half-width (HWHM) and shift in the distribution, respectively, due to Van der Waals broadening and Stark broadening, and $\alpha$ is the ion broadening parameter. The microfield strength distribution is due to ion Stark broadening and is complicated to calculate. However, 
the effects of ion Stark broadening can be estimated using Eq. 224 from Spectral Line Broadening by Plasma by Hans Griem [118]. The calculation for the $794.8176 \mathrm{~nm}$ neutral argon line shows that $\sim 5 \%$ of Stark broadening is due to ions (the rest being due to electrons) [122]. Therefore, ion Stark broadening was ignored $\left(W_{R}(\beta)=1\right.$ and $\left.\alpha=0\right)$, resulting in an asymmetric tail of the model line shape.

To form the theoretical spectrum, the Gaussian and Lorentzian distributions are convolved to form a Voigt profile.

$$
f_{\text {Voigt }}(\lambda)=\int_{-\infty}^{\infty} f_{\text {Gauss }}(\lambda-y) f_{\text {Lor }}(y) d y
$$

For the $794.8176 \mathrm{~nm}$ argon line in VADER, the profile has the form

$$
f_{\text {Voigt }}(\lambda)=\frac{1}{\sigma \pi \sqrt{2 \pi}} \int_{-\infty}^{\infty} e^{\frac{-\left(\lambda-y-\lambda_{0}\right)^{2}}{2 \sigma^{2}}} \frac{1}{1+\left[\left(y-\lambda_{0}-d_{c}\right) / w_{c}\right]^{2}} d y
$$

The theoretical profile is then fit to the experimental line profile, which gives values for $\sigma, w_{c}$ and $d_{c}$. For this work, a Matlab program was written and the line was fitted using using a built-in non-linear regression tool (function lsqcurvefit). The deconvolution process separates the Gaussian and the Lorentzian contributions, which allows the separation of broadening terms with a Gaussian profile (for instance, instrumental broadening and Doppler broadening) from those with a Lorentzian profile (for instance, Stark and Van der Waals broadening).

This deconvolution method does not separate terms that have similar functional forms. Therefore, it is still necessary to separate the Van der Waals and Stark contributions. For a Lorentzian, the sum of the HWHM of each broadening term approximately adds up to the 
total HWHM of the Lorentzian profile. Thus,

$$
w_{S t}=w_{c}-w_{V D W}
$$

was used to calculate the HWHM of the Stark distribution. $w_{V D W}$ is calculated analytically using Equation 4.4 and transition data from the NIST atomic spectra database [113, 123]. To solve for the electron density, Equation 4.11 is solved for $N_{e}$. The electron impact widths and wavelength shifts for multiple atomic transitions for varying plasma temperatures are readily available $[115,118,124,125]$. Note, the widths in these references are usually given at multiple temperatures and are for electron densities of either $10^{16}$ or $10^{17} \mathrm{~cm}^{-3}$. Therefore, it is important that the electron temperature of the plasma be well known to achieve accurate plasma densities. Note, reference HWHM values may need to be scaled to $10^{16} \mathrm{~cm}^{-3}$ (if ion contributions are ignorable, the HWHM scales linearly with density) to be used in Equation 4.11. Note, this method of finding the Stark HWHM makes several simplifications and assumptions; a more rigorous mathematical analysis was presented by Nikolic [126].

The Stark broadening experiments were done with a pure argon plasma while using the TREK power supply. The DBD had a gap distance of $1 / 8$ ", a $0.025 "$ piece of alumina as the dielectric and a spectrometer slit size of 10 microns. The tests were done at power supply frequencies of $2 \mathrm{kHz}$ with voltages of $4 \mathrm{kVp}-\mathrm{p}$ (6.5 W forward power), $2 \mathrm{kHz}$ at $5 \mathrm{kVp}-\mathrm{p}(10.8$ W), $4 \mathrm{kHz}$ at $2 \mathrm{kVp}-\mathrm{p}(12.9 \mathrm{~W})$ and $4 \mathrm{kHz}$ at $5 \mathrm{kVp}-\mathrm{p}(14.1 \mathrm{~W})$. The lower voltages were possible due to the low breakdown voltages of argon and the higher electric fields allowed by the thin alumina dielectric. An example of one of the $794.8176 \mathrm{~nm}$ neutral argon spectra along with a fitted line profile is shown in Figure 4.3. The spectra at each plasma setting was approximately the same and shows that the fitting program does a good job of matching 
the emission line shape. However, in each of the experimental profiles there was a small amount of asymmetry at the wings (skewed towards higher wavelengths). The asymmetry is most likely due to a combination of ion Stark broadening, the Stark shift and Doppler broadening (it is possible that there is a small population of high energy ions/neutrals in a DBD plasma) [76].

Despite different voltages and frequencies, the results of the deconvolutions consistently gave a Lorentzian HWHM of $\sim 0.032 \mathrm{~nm}$. Using Equation 4.11, $w_{V D W}$ was calculated to be $\sim .034 \mathrm{~nm}$ (doesn't change with voltage or frequency), where $\lambda_{0}=794.8176 \times 10^{-7} \mathrm{~cm}$, $N_{p}=2.40886 \times 10^{19} \mathrm{~cm}^{-3}$ (particle density at atmospheric pressure), $\bar{\alpha}=1.654 \times 10^{-24}$ $\mathrm{cm}^{3}, T_{g}=300 \mathrm{~K}, \mu_{P E}=19.97 \mathrm{amu}(\mathrm{Ar}-\mathrm{Ar}), E_{\text {Ion }}=15.7595 \mathrm{eV}(\mathrm{Ar}), E_{U}=13.2826 \mathrm{eV}$, $l_{U}=1(4 \mathrm{p}), E_{L}=11.7232 \mathrm{eV}$ and $l_{L}=0(4 \mathrm{~s})$. Solving Equation 4.17, gives a Stark width of $\sim-0.002 n m$, which signifies that the broadening ratio $\left(w_{S t} / w_{V D W}\right)$ is much less than 1. This broadening ratio is consistent with theory, the theoretical $w_{S t}$ for the $794.8176 \mathrm{~nm}$ is approximately $0.000361 \mathrm{~nm}$ (from [118] and scaled to the maximum DBD electron densities, $\left.\sim 10^{15} \mathrm{~cm}\right)$, which gives a broadening ratio of $\sim 0.01$. The error involved with a Stark broadening measurement is at least $20 \%$ in well tested systems [113]. Therefore, to find an accurate electron density a broadening ratio of at least unity or higher is required. Which means it was not possible to find the electron density using the $794.8176 \mathrm{~nm}$ neutral argon line in a traditional atmospheric pressure DBD.

There are several methods to increase the broadening ratio. The first method is to choose emission lines which are more strongly affected by the Stark effect. For VADER, this would require an emission line with a Stark width greater than 1 angstrom $(\AA)$ at $10^{16} \mathrm{~cm}^{-3}$ [118]. Lines with such large Stark widths in argon are very dim (they have an intensity 100 times lower than the $794.8176 \mathrm{~nm}$ line) and were not observable in VADER. 
Another solution is to add a dopant species, usually less than $1 \%$ of the total gas. A popular additive for Stark broadening is hydrogen, because the hydrogen Balmer lines are well-documented and have relatively large Stark HWHM [127-129]. Note, any dopant can change a plasma's chemistry and dynamics, so care must be taken when choosing the additive. Another method for improving the broadening ratio is to reduce the amount of Van der Waals broadening, which is accomplished by either choosing a shorter wavelength or reducing the neutral gas pressure. Argon has a large number of transitions at lower wavelengths, however the brightest ones ( 100 times lower intensity than the 794.8176) have even weaker Stark coupling $[115,118,123]$. As for lowering the neutral pressure, changing the pressure drastically changes properties of a plasma (density, temperature,discharge mode, etc). Considering this work was only concerned with $\mathrm{DBD} \mathrm{CO}_{2}$ dissociation at atmospheric pressure, this was not attempted. Similarly, increasing the electron density would improve Stark broadening, however that was also beyond the scope of this work. 


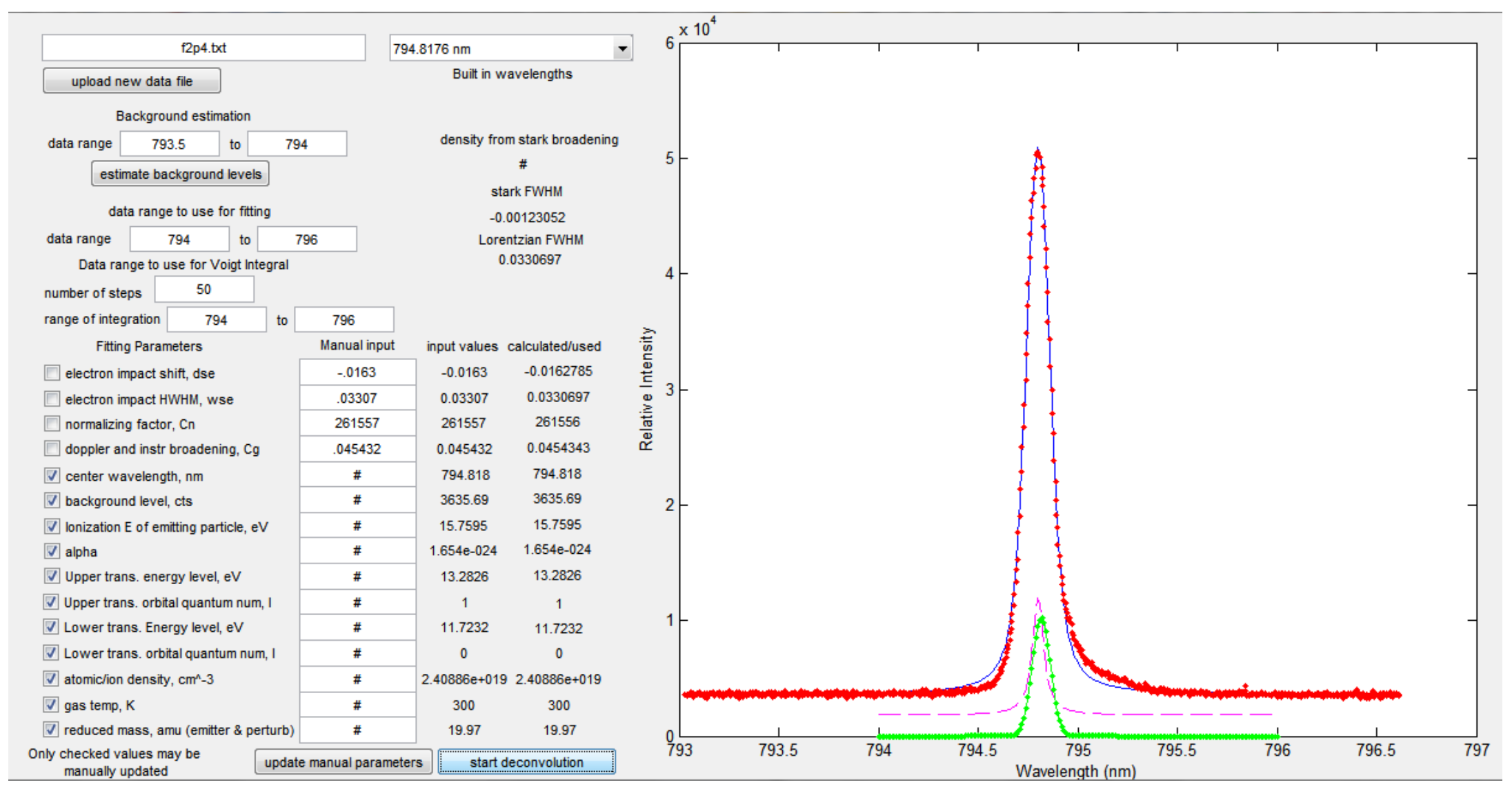

Figure 4.3: Deconvolution results for the $794.8176 \mathrm{~nm}$ neutral argon line The red dots are the measured line shape. The dashed magenta line and the green dotted-line are the asymmetric Lorentzian and Gaussian profiles which were convolved to form the the blue line which is fitted to the measured spectra. 
Stark broadening spectroscopy was not possible in VADER, but the technique can be similarly applied to Van der Waals broadening to estimate either the neutral gas temperature or the neutral density. Van der Waals broadening spectroscopy requires knowledge of either the gas temperature or the neutral density to find the other. Since Van der Waals broadening was the only significant Lorentzian broadening mechanism in VADER and the neutral density was externally set (atmospheric pressure, $2.4088 \times 10^{19} \mathrm{~cm}^{-3}$ ), the electron temperature was found by equating equation 4.4 with the fitted Lorentzian HWHM. Using the emission profiles used for Stark spectroscopy, the neutral temperature was calculated to be $\sim 260 \mathrm{~K}$ for each plasma setup. The neutral temperature of DBDs is usually considered to be room temperature $(\sim 300 \mathrm{~K}$ in VADER). This gives an error of $\sim 13.3 \%$ ((actual measured)/actual). The error in the Van der Waals fitting is expected to be similar to that of Stark spectroscopy with an error $>20 \%$ with an actual error more likely to be in the range of $30-40 \%$, therefore the error is well within the expected range for this diagnostic. Which means even though the forward power changed with each experiment, the neutral temperature was found not to change in any significant manner. 


\section{Chapter 5}

\section{VADER $\mathrm{CO}_{2}$ Dissociation}

\section{Experiments}

The main focus of the VADER experiment was to look at the efficiency of $\mathrm{CO}_{2}$ decomposition using a DBD, test the different variables that affect decomposition, and then explain the effects of these variables using the theory and diagnostics discussed. The first parameter tested was the dielectric material. The goal was to find the material that yielded the highest electron density and energies in a DBD. It was initially believed that this would lead to the best plasma dissociation parameters. Once the appropriate dielectric was selected (quartz), the VADER $\mathrm{CO}_{2}$ dissociation experiments were begun. The experiments entailed testing several variables for their effectiveness at dissociating $\mathrm{CO}_{2}$ in a DBD including voltage, frequency, pulse mode, waveform, gas flow rates, gas mixtures and the inclusion of a catalyst. Each run was done in VADER and used the reaction box described in Section 3. The results show that for the VADER parameters, $\mathrm{CO}_{2}$ dissociation efficiency is highly dependent on the interplay between power supply frequency, voltage and the gas composition. Additionally, 
it was discovered that a properly chosen catalyst can highly influence chemical reactivity within a DBD. The effects of gas flow rate and pulse modes on $\mathrm{CO}_{2}$ dissociation were found to be minor.

\subsection{Dielectric Tests}

The first set of experiments in VADER involved testing different dielectric materials and thicknesses of dielectric. Dielectric materials have a large effect on the electric field within the air gap of a DBD, so they play a key role in determining the best discharge parameters. The chosen material also has several more subtle effects on the discharge, such as adding to the electron population through secondary emission and acting as a catalyst to either help or hinder the desired plasma chemistry [130-132]. Considering the high energy endothermic reactions required for $\mathrm{CO}_{2}$ dissociation, the best parameters were considered to be those that gave the highest electron energies, densities and discharge volumes. These parameters were found through a visual inspection of the plasma, as the electron energy and density are directly related to the brightness of the plasma and the density of streamers was visible in VADER. 


\begin{tabular}{|c|c|c|c|c|c|}
\hline Material & $\begin{array}{l}\text { Max Temp } \\
{ }^{\circ} \mathrm{C}\end{array}$ & dielectric constant & $\begin{array}{l}\text { dielectric strength } \\
\mathrm{V} / \mathrm{mm}\end{array}$ & chemical makeup & source \\
\hline Macor & $\begin{array}{l}800 \text { cont } \\
1000 \text { pulsed }\end{array}$ & $6.0 @ 1 \mathrm{kHz}$ & $40 @ \mathrm{AC}$ & $\begin{array}{l}46 \% \mathrm{SiO}_{2} 17 \% \mathrm{MgO} 16 \% \mathrm{Al}_{2} \mathrm{O}_{3} \\
10 \% \mathrm{~K}_{2} \mathrm{O} 7 \% \mathrm{~B}_{2} \mathrm{O}_{3} 4 \% \mathrm{~F}\end{array}$ & {$[133]$} \\
\hline Lava & 1000 & $5.3 @ 1 \mathrm{MHz}$ & 3.15 & $\mathrm{Al}_{2} \mathrm{O}_{3}$ and $\mathrm{SiO}_{2}$ & [133] \\
\hline Boron Nitride & 1800 & 4-4.6@8.8 GHz & $67-95 \mathrm{AC}$ & $\mathrm{BN}_{3}$ & {$[133]$} \\
\hline Fused Quartz & 1000 & $\begin{array}{l}3.91 @ 1 \mathrm{kHz} \\
3.75 @ 1 \mathrm{MHz}\end{array}$ & $25-40$ & $\mathrm{SiO}_{2}$ & {$[134]$} \\
\hline Alumina & 1700 & $8.4-10.5$ & $9-10$ & $\mathrm{Al}_{2} \mathrm{O}_{3}$ & [133] \\
\hline Kapton & 450 & $3.1-3.5$ & $\begin{array}{l}7700 @ 1 \mathrm{mil} \\
3900 @ 5 \mathrm{mil}\end{array}$ & organic polymer & {$[135]$} \\
\hline
\end{tabular}

Table 5.1: Properties of different dielectric materials tested in VADER. Max Temp is the maximum working temperature of the material 
A list of the tested dielectric materials are given in Table 5.1. The four listed properties were the determining factors for choosing these materials, of which the dielectric constant and dielectric strength are the most important for a DBD. A higher dielectric constant corresponds to a larger electric field within the air gap (assuming no geometric change in the system) and a larger amount of dielectric heating. Dielectric heating is due to the rotation of particles in a material when an oscillating electric field is applied; during each rotation a small amount of energy is converted to heat. The dielectric strength determines the amount of voltage that can be applied to the material before it breaks down. The maximum working temperature is important if the material is to be used in high temperature situations, which is often the case in $\mathrm{CO}_{2}$ producing systems. The chemical makeup of a material is important if there is worry of sputtering or contamination in the system. Each material was tested for sputtering using a pure Ar plasma and looking for the material constituents using the RGA system. No sputtering was detected.

Initial experiments were performed on macor, boron nitride, and quartz to test their discharge qualities. A $0.0625 "(1 / 16 ")$ slab of each material was placed between the electrodes with various gas fills at atmospheric pressure $\left(100 \% \mathrm{CO}_{2}, 90 \%, 80 \%\right.$ and $70 \%$. Ar was the remaining fill), electrode distances $(1 / 16 ", 1 / 8 ", 3 / 16 ")$, voltages $(0-20 \mathrm{kVp}-\mathrm{p})$ and power supply frequencies $(0-10 \mathrm{kHz}$, TREK power supply). The maximum power and a visual inspection of the discharge was recorded. The visual inspection included observing the brightness of the plasma, the density of streamers, the type of streamers (for instance, a single streamer formed, many individual streamers formed, etc.) and the range of frequencies and voltages in which a discharge formed-the results are provided in Appendix A. These experiments demonstrated that quartz had the brightest and most uniform discharge with the largest range of frequencies and voltages over which a discharge formed. The next most 
effective material was boron nitride followed by macor.

Another major factor in plasma performance is the dielectric thickness. The effects of the thickness were seen when the same analysis as above was attempted on 0.001", 0.002" and $0.005 "$ thick pieces of Kapton. Kapton showed a much brighter discharge than any of the other tested materials, but with far fewer streamers. These tests could not be done as rigorously as the above tests due to the eventual breakdown of the material. Breakdown consisted of the plasma burning a small hole in the material and subsequently forming an arc plasma. The $0.005 "$ pieces lasted one hour at the highest voltage settings and thinner pieces broke down even sooner. For this reason, Kapton was not used for the $\mathrm{CO}_{2}$ dissociation experiments. After beginning the $\mathrm{CO}_{2}$ dissociation experiments using quartz, a .025" thick piece of alumina was tested and found to be both brighter with the same discharge volume as the quartz cases. But, for consistency, quartz was used for the rest of the $\mathrm{CO}_{2}$ dissociation experiments.

These results imply that a thinner dielectric and a smaller dielectric constant create a denser and higher energy plasma. However, a smaller dielectric constant runs counter to literature $[73,131,136]$. Therefore, a second round of testing was performed, comparing a $0.0625 "(0.16 \mathrm{~cm})$ slab of alumina $\left(\epsilon_{r}=8.4-10.5\right)$ to a $0.06 "$ slab of quartz $\left(\epsilon_{r}=3.91\right)$. These measurements were conducted in air using the DIDRIV10 power supply while changing the air gap distances (1/16" and 1/8"), voltages (100\% and $90 \%)$, and power supply frequencies $(28.4 \mathrm{kHz}$ and $30 \mathrm{kHz}$ ) ; the details are provided in Appendix A. For each run, in addition to the visual inspection discussed above, the voltage, current, power and optical emission spectrum were recorded. Voltage, current and power were found using the voltage divider and current monitors as discussed in Section 3.2.3. The emission spectrum was recorded using the Mcpherson scanning monochromator and the collection optics as described in Section 
3.2.2. Line intensities were measured at $399.5 \mathrm{~nm}$ (N II line), $777 \mathrm{~nm}$ (O I triplet) and 844.6 nm (O I triplet) with an integration time of 20 seconds. The amplitude of the emission lines is representative of the combination of electron densities and temperatures in the DBD, as the ion energies are comparably small.

The emission lines showed that both dielectrics had relatively similar emission intensities. The intensities were skewed in both the $777 \mathrm{~nm}$ and $844.6 \mathrm{~nm}$ lines due to those lines overlapping with molecular bands (which suggests that differences in material affect the excitation of various molecular bands), however the $399.5 \mathrm{~nm}$ line had no such interference. The line intensities for the 399.5nm line are shown in Figure 5.1. On average the difference in amplitudes were $0.03 \%$ with a standard deviation of $4.0 \%$, which implies that both plasmas are reaching similar electron energies and densities, thus electric fields as well. However, the alumina was found to require on average $30 \%$ less forward power and had a slightly smaller streamer volume when compared to quartz. The difference in volume is small in comparison to the power difference, therefore it is expected that this excess energy is lost due to a combination of dielectric heating and resistive power losses.

The electric field within the air gap of a parallel plate capacitor is

$$
E=\frac{\epsilon_{r} \Delta V}{\epsilon_{r} d_{a i r}+d_{\text {diel }}}
$$

Where $E$ is the electric field, $\epsilon_{r}$ is the dielectric constant/relative permittivity of the dielectric, $\Delta V$ is the applied voltage, $d_{\text {air }}$ is the width of the air gap, and $d_{\text {diel }}$ is the thickness of the dielectric. A lower dielectric constant means a smaller electric field is applied to the air gap for a given voltage and a higher voltage is required for gas breakdown. Plugging in for VADER $\left(d_{\text {air }} \sim 0.15875 \mathrm{~cm}\right)$ using alumina $\left(\epsilon_{r}=9, d_{\text {diel }} \sim 0.15875 \mathrm{~cm}\right)$ and Quartz $\left(\epsilon_{r}\right.$ 

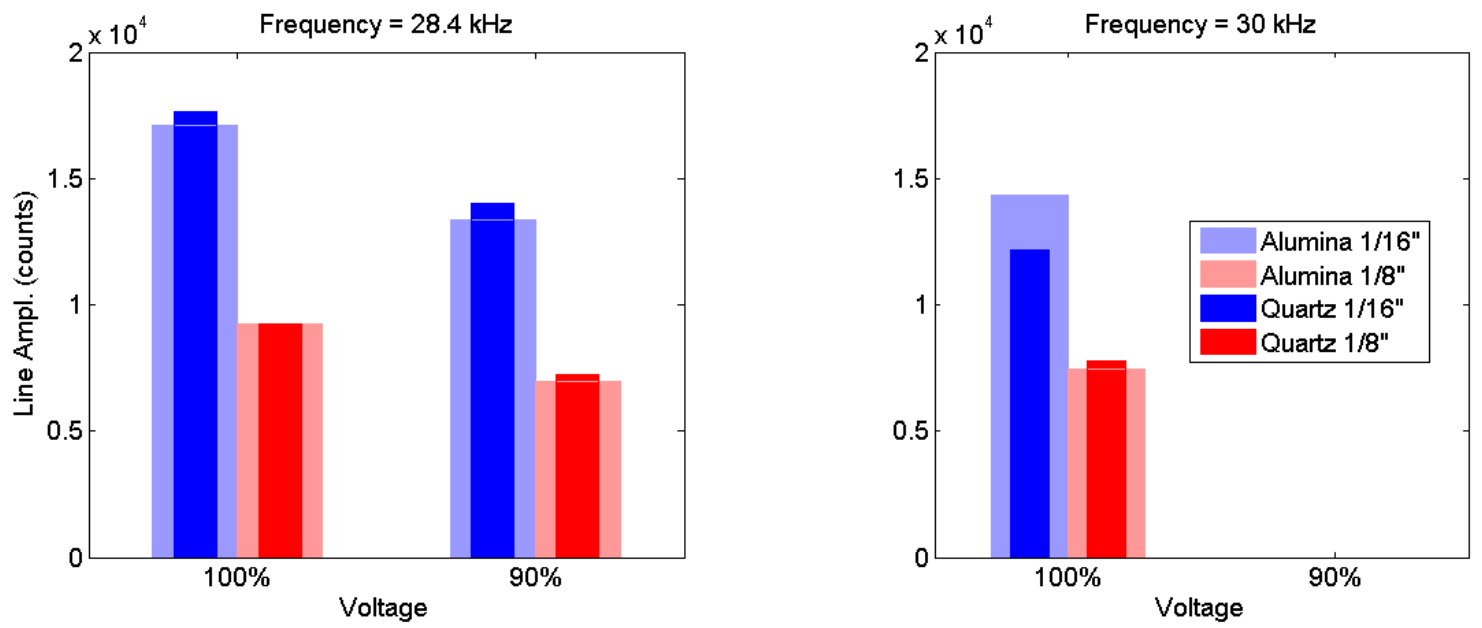

Figure 5.1: $399.5 \mathrm{~nm}$ line amplitudes when using Alumina and Quartz Dielectrics. The $399.5 \mathrm{~nm}$ line amplitude versus material versus the width of the air gap versus voltage $(90 \%$ and $100 \%$ ) for the frequencies $(28.4 \mathrm{kHz}$ and $30 \mathrm{kHz})$. The emission amplitudes at $90 \%$ voltage and $30 \mathrm{kHz}$ were eliminated, because the streamers were not within the line of sight of the optics.

$=3.91, d_{\text {diel }} \sim 0.1524 \mathrm{~cm}$ ), gives an electric field of $E \sim 5.67 \Delta V$ and $5.06 \Delta V$, respectively. Therefore, for quartz to have the same electric field as alumina it would require $\sim 10 \%$ higher voltage. Yao et al. and Pipa et al. showed that the equivalent circuit diagram for a streamer DBD is two capacitors (one for the dielectric and one for the air gap) and a resistor (internal resistance) in series $[137,138]$. Streamers in the circuit are represented by a switch which shorts the air gap capacitor with a resistor (representing the resistance of the streamer/particle energy gain) when the voltage across the air gap is above the breakdown voltage of the gas. Since the breakdown voltage is kept constant, the power loss (and discharge parameters) across the air gap $\left(P=V^{2} / R\right)$ is unaffected by the increase in voltage. However, power loss across the internal resistor increases following $P=V^{2} / R$. Therefore, an increase in voltage of $10 \%$ leads to a $21 \%$ increase in power loss to the internal system, which could explain the difference in forward power despite no change in the discharge. An additional effect of higher voltages is an increased likelihood of creating corona discharges along the 
power cables, which may contribute to the observed increase in power losses.

A smaller source of power loss comes from dielectric heating, which obeys the equation [139]:

$$
P_{\text {DielHeat }}=\omega \epsilon_{0} \epsilon_{r} \tan \delta|E|^{2}
$$

where $P_{\text {DielHeat }}$ is the power loss per unit volume due to dielectric heating, $\omega$ is the power supply frequency, $\epsilon_{0}$ is the permittivity of free space $\left(8.854 \times 10^{-12} \mathrm{Fm}^{-1}, \epsilon_{r}\right.$ is the dielectric constant of the material, $\tan \delta$ is the dissipation factor of the material and $|E|$ is the magnitude of the electric field. At low frequencies, dielectric heating is usually very small and is ignorable, but at the high voltages used in VADER dielectric heating is non-negligible. Plugging in for quartz and alumina $\left(\epsilon_{r} \sim 3.98\right.$ and 9 , respectively) with an electric field of $\sim 31.5 \mathrm{kV} / \mathrm{cm}(5 \mathrm{kV} / .0625 \mathrm{inch})$ and a frequency of $2 \pi \times 28.4 \mathrm{kHz}$ and a dissipation factor of $\sim 4 \times 10^{-4}$ for alumina and $\sim 1 \times 10^{-4}$ for quartz, gives dielectric heating losses of $\sim 56438$ $\mathrm{W} / \mathrm{m}^{3}$ and $\sim 6240 \mathrm{~W} / \mathrm{m}^{3}$, respectively. Taking into account the dielectric volume $(\sim 3.2$ $\mathrm{cm}^{3}$ ), the expected power losses are $0.18 \mathrm{~W}$ and $0.02 \mathrm{~W}$, respectively. These energies are not enough to account for the observed power difference, but at large AC voltages dielectrics have a material dependent increase in the dissipation factor, called tipping up [140, 141]. Tipping up can increase the loss factor by well over a factor of 10 and suggests a possible reason for further differences in forward power for the two materials.

The second set of dielectric tests imply that dielectrics with a higher dielectric constant are more efficient at creating a plasma than materials with a lower dielectric constant, which is consistent with literature. However, it should be noted that these results do not take into account any properties of the dielectrics beyond the dielectric constant, such as secondary electron emission or surface chemistry. Secondary emission is important for electron seeding 
of the plasma and could cause the decrease in breakdown voltages seen in these experiments [142-144]. Also, if the material has any reactivity with the gas, it can either hinder or improve certain reaction rates. A change in the reaction rates changes the gas composition, which can have significant effects on the plasma dynamics. The effects of the DBD material on the chemistry were shown by Li et al. and in Section 5.4.7 of this work [136].

Another result of the second set of dielectric testing was the significant difference in the emission intensities when the discharge gap was changed. As seen in Figure 5.1, the 1/16" discharge gap was consistently approximately $85 \%$ brighter than the $1 / 8$ " discharge gap despite using $50 \%$ less power. The difference in power is most likely due to the difference in the discharge voltages (due to the change in the air gap) and the reduction in power lost to internal resistance. The increase in emission is most likely due to the lower discharge voltage, but it is unclear if this occurred due to an increase in the number of streamers, a change in the plasma temperature (due to a change in the plasma kinetics, because of the

shorter discharge distance) or an increase in the streamer volume within the line of sight of the optics (more of the surface discharge is within the line of sight).

\section{$5.2 \quad \mathrm{CO}_{2}$ Dissociation Experimental Setup}

The dissociation efficiency of VADER was measured using the calibrated results from the RGA system described in Section 3.2.1. The RGA measured the partial pressures of $\mathrm{CO}_{2}$ $(E / m=44), \operatorname{Ar}(E / m=40), \mathrm{CO}\left(E / m=28\right.$, which also corresponds to $\left.\mathrm{N}_{2}\right), \mathrm{O}_{2}(E / m=32)$, $\mathrm{H}_{2} \mathrm{O}(\mathrm{E} / \mathrm{m}=18)$ and $\mathrm{H}_{2}(\mathrm{E} / \mathrm{m}=2)$. The last two partial pressures were used to detect leaks in the system.

An experimental run consisted of setting up the system for one of the combination of 
variables listed in Table 5.2, then the plasma was turned on for 5 minutes then shutoff with a 5 minute plasma off period both before and after. To minimize run time, the 5 minute off times were often shared with other runs. During this 15 min run time, the RGA would record the partial pressures of each species at four different times: a short time before the plasma was turned on (begin off), a short time after it was turned on (begin on), before the plasma was turned off (end on) and after the plasma was turned off (end off). An example of the RGA data is in Figure 3.13. The measurements consisted of the partial pressures, plus each of the system variables and a power reading, as explained in Section 3.2.3.

\begin{tabular}{|lll|}
\hline Variable & Tested Parameters & \\
\hline Power supplies & TREK 10/10B & DIDRIV10 \\
Voltage & $20 \mathrm{kVp}-\mathrm{p}$ & $90 \%$ and $100 \%$ of max voltage $(\sim 2-3 \mathrm{kVp}-\mathrm{p})$ \\
Frequency & $0-5 \mathrm{kHz}(.5 \mathrm{kHz}$ steps $)$ & $28.4 \mathrm{kHz}$ and $30 \mathrm{kHz}$ \\
Pulsed modes & continuous & continuous, $3.6 \mathrm{~ms}$ on 0.5 off, $2.4 \mathrm{~ms}$ on 2.4 off \\
Waveform & square wave & sinusoidal \\
\hline Gas flow rate & $200 \mathrm{SCCM}, 300 \mathrm{SCCM}$ \\
Gas mix & $100 \% \mathrm{CO}_{2}, 60 \% \mathrm{CO}_{2}$ and $40 \% \mathrm{Ar}$ \\
Catalyst & no catalyst, $\mathrm{TiO}_{2}$ & \\
Temperature & $20^{\circ} \mathrm{C}$ \\
Pressure & 760 Torr $(1 \mathrm{~atm})$ & \\
\hline
\end{tabular}

Table 5.2: The variables tested during the $\mathrm{CO}_{2}$ dissociation experiments in VADER. Each experiment had the dielectric box installed with a $1 / 16$ " thick quartz disk as the dielectric and a 2" diameter by $1 / 16$ " height discharge area. The non-linear relationship between power supply voltage and frequency is discussed in Section 3.1.2.

Experiments were performed in two sets, the first set used the TREK power supply and the second used the DIDRIV10 power supply; the power supplies are discussed in Section 3.1.2. Beyond the difference in power supply parameters (Listed in Table 5.2), the experimental setups were the same, except for two minor differences. The TREK power supply used a square wave voltage output at frequencies below $2.5 \mathrm{kHz}$, whereas the DIDRIV10 power supply had a sinusoidal output. Initial experiments with the TREK power supply 
showed that the square wave dissociated more $\mathrm{CO}_{2}$ than the sinusoidal signal, therefore it can be said that the TREK results are the best case scenario results for the TREK power supply. The other minor difference was the reaction box that was developed during the TREK experiments. These changes should have a small effect on the results considering the discharge area, materials and spacing for the reaction box were not changed during the modification process.

\subsection{Calculations and Error}

\subsubsection{Energy Efficiency, Dissociation Rate and Percent Difference}

To find the efficiency of a chemical reactor, it is important to know how much of the input gas was reacted (dissociation rate) and how much of the input energy went towards the chosen reaction (energy efficiency). The first step to finding the energy efficiency and dissociation rate was calculating the partial pressure of each gas in the reactor for the four RGA readings using the calibration data, as described in Section 3.2.1. Using the reactor partial pressures the average change in pressure between when the plasma was on and off was calculated.

$$
\Delta p_{b, e}=p_{\text {on }}-p_{\text {off }} \quad \Delta p_{\text {ave }}=\frac{\Delta p_{\mathrm{b}}+\Delta p_{\mathrm{e}}}{2}
$$

Where, $\Delta p_{b, e}$ is the change in the reactor partial pressure for the chosen gas for the before (b) cases or end (e) cases, $p_{x}$ is the reactor partial pressure for the chosen gas (on is for the plasma on case and off is the off case), $\Delta p_{\text {ave }}$ is the average change in reactor partial pressure. 


\section{$\mathrm{CO}_{2}$ Dissociation Rate}

The dissociation rate is the number of dissociations per unit of time and was found by plugging the average reactor partial pressure into the ideal gas law $(\mathrm{PV}=\mathrm{NRT})$ :

$$
\begin{gathered}
C \mathrm{O}_{2} \& C O: \frac{d N_{\mathrm{CO}_{2}}}{d t}=\frac{u \Delta p}{R T} \\
\mathrm{O}_{2}: \frac{d N_{C O_{2}}}{d t}=2 \times \frac{u \Delta p}{R T}
\end{gathered}
$$

where $d N / d t$ is the dissociation rate (number of $\mathrm{CO}_{2}$ molecules dissociated per unit time), $u$ is the volume of gas flowing through the reaction zone per unit time (aka the gas flow rate), $R$ is the ideal gas constant, $T$ is the system temperature. Oxygen has an additional factor of 2 because for every one $\mathrm{O}_{2}$ particle created two $\mathrm{CO}_{2}$ particle are dissociated, as per Equation 2.5. From the dissociation rate, the percentage of the input $\mathrm{CO}_{2}$ dissociated was calculated following

$$
\% \text { Diss }=\frac{d N_{C O_{2}}}{d t} / \dot{N}_{C O_{2}}
$$

where $\dot{\mathrm{N}}_{\mathrm{CO}_{2}}$ is the $\mathrm{CO}_{2}$ particle flow rate.

\section{Energy Efficiency}

The energy efficiency $(\eta)$ is the ratio of the amount of energy that went towards a specific reaction/s and the total input energy. The first step to finding the energy efficiency of $\mathrm{CO}_{2}$ dissociation was to find the number of molecules converted per unit of energy,

$$
\frac{d N_{C O_{2}}}{d E}=\frac{d N_{C O_{2}} / d t}{P}
$$


where $\frac{d N_{C O_{2}}}{d E}$ is the number of molecules of $\mathrm{CO}_{2}$ converted per unit energy and $P$ is the forward power. The energy efficiency is found by multiplying the minimum energy required to dissociate a $\mathrm{CO}_{2}$ molecule, $2.94 \mathrm{eV} /$ molec (discussed in Section 2.1), by the number of molecules converted per unit energy

$$
\eta=2.94\left[\frac{e V}{\text { molec }}\right] \times \frac{d N}{d E} \times 100
$$

The number of molecules converted per unit of energy is also a good metric for the efficiency of a chemical system. However, when looking at a specific chemical reaction, the energy efficiency is a better metric as it is based on the specific energy requirements of the reaction. The atomic efficiency is also an important quantity in many chemical reactor studies. Atomic efficiency is the ratio of the molecular weight of desired products and the total molecular weight of the reactants; it identifies the number of wasted or unused atoms in a chemical system. However, due to the simplicity of $\mathrm{CO}_{2}$ dissociation chemistry, the energy efficiency and the number of molecules dissociated per unit of time is sufficient for understanding the efficiency of these reactions.

Percent Difference (Percent Change) The percent difference is the change in energy efficiency or dissociation rate due to a single experimental variable. For instance, the percent difference in energy efficiency due to the addition of a catalyst is calculated using:

$$
\% \text { Diff }=\frac{\eta_{\text {Catalyst }}-\eta_{\text {No Catalyst }}}{\left(\eta_{\text {Catalyst }}+\eta_{\text {No Catalyst }}\right) / 2} \times 100
$$

where $\eta_{\text {Catalyst }}$ is the average energy efficiency for one experimental setup using a catalyst, $\eta_{\text {No Catalyst }}$ is the average energy efficiency of the same experimental setup, except without 
a catalyst. This calculation gives the difference in energy efficiencies between one case, during which all variables were the same, except one used a catalyst and the other didn't. By applying this calculation to every combination of variables a statistical distribution of the effects of the catalyst was created. This calculation was applied to each set of system variables for both the energy efficiency and dissociation rate, the results of which are plotted as bar graphs with a bin size of $2.5 \%$. The mean, median and skew were calculated for each distribution to show both whether the results were statistically significant and the level of variability in the data.

\subsubsection{Error}

The two major sources of error in the above calculations come from the measurement of the forward power and the RGA partial pressures. To reduce error in the measurement of forward power the IV curves were averaged over 100 cycles. Figure 3.5 shows examples of the current and voltage curves found during experiments and the associated power curve. After averaging, the error in power was $\sim 4 \%$ or 2 watts, based on the fluctuations in the power readings over several hours of run time. This error is negligible in comparison to the RGA error and contributed to less than $6 \%$ of the total error.

The RGA error is due to small oscillations in RGA readings and a slow drift in partial pressure, the origins of which are discussed in Section 3.2.1. These errors are not significant when looking at the partial pressures of each gas, but become significant when calculating the difference in partial pressures, Equation 5.3. To find the error due to the small oscillations in RGA readings, the range of the oscillations were collected at multiple times over multiple data sets and the average oscillation amplitude was used as the error. This method gave

an error of $0.038 \times 10^{-8}$ Torr $(\sim 1.6 \%)$ in $\mathrm{O}_{2}$ partial pressure measurements and $0.025 \times 10^{-7}$ 
Torr $(\sim 1 \%)$ in CO measurements. For the error due to drift, the standard deviation of the calibration runs was used as the error, which gave an error of $0.34 \times 10^{-8}$ Torr $(\sim 14 \%)$ for $\mathrm{O}_{2}$ and $0.14 \times 10^{-7}$ Torr $(\sim 4.5 \%)$ for $\mathrm{CO}$ at the recorded partial pressures. However, the error due to drift in $\mathrm{CO}$ is an average of only two calibration runs (limited CO runs were performed because of the safety hazards associated with $\mathrm{CO}$ experiments) and was considered to be too small. After discussing this issue with engineers at VTI, it was decided that an error of $0.31 \times 10^{-7}$ Torr $(10 \%)$ is a more realistic value [145]. Combining both the error due to small oscillations and the drift error, gave a final RGA error $\left(\sigma_{\mathrm{RGA}}\right)$ of $0.342 \times 10^{-8}$ Torr for $\mathrm{O}_{2}$ and $0.311 \times 10^{-7}$ Torr for CO.

The final propagated RGA error is shown in the dissociation rate graphs and the propagated RGA error combined with the error in power in each of the energy efficiency graphs. As the values in the dissociation rate graphs are the mean of all experimental runs with the same experimental setting, the error was calculated using the error in the mean of those experimental runs. The energy efficiency uses the dissociation rate in its calculation. Therefore, the error in the energy efficiency was calculated using both the error in the mean and the error in the forward power, following

$$
\sigma_{\eta}=\eta \sqrt{\left(\frac{\sigma_{p}}{p}\right)^{2}+\left(\frac{\sigma_{P}}{P}\right)^{2}}
$$

where, $\sigma_{\eta}$ is the error in the energy efficiency, $\sigma_{p}$ is the error in the mean of the partial pressures and $\sigma_{P}$ is the error in the forward power.

The percent difference compares the energy efficiency and $\mathrm{CO}_{2}$ dissociation rate for different experimental setups. Because different experiments are being compared, the error in the mean is not an appropriate metric. Instead, the standard deviation and the skew of the 
distribution are used. The standard deviation provides a measure of the consistency of the change in energy efficiency and dissociation rate across many different experiments and the skew gives a metric of the symmetry of the data. The skewness of each distribution was calculated using the sample skewness [146],

$$
g 1=\frac{\frac{1}{n} \sum_{i=1}^{n}\left(x_{i}-\bar{x}\right)^{3}}{\left(\frac{1}{n} \sum_{i=1}^{n}\left(x_{i}-\bar{x}\right)^{2}\right)^{3 / 2}}
$$

where $g 1$ is the sample skewness, $n$ is the number of data points in the distribution, $x_{i}$ is the value of each data point and $\bar{x}$ is the average of the distribution. Because the data are a sample population, $g 1$ is biased. To compensate for this bias, $G 1$ was used as the skew of the distribution,

$$
G 1=g 1 \times \frac{\sqrt{n(n-1)}}{n-2} .
$$

Negative $G 1$ values mean the distribution has negative skew (most data points lie to the right of the distribution) and positive $G 1$ means the distribution has positive skew. According to Bulmer, "Principles of Statistics" the rule of thumb for skewness is that a value of G1 equal to -1 or lower or equal to +1 or greater indicates a large amount of skew, -1 to $-1 / 2$ and $+1 / 2$ to +1 denotes moderate skew and $-1 / 2$ to $+1 / 2$ is approximately symmetric [147].

\section{$5.4 \quad$ Experimental Results}

An overview of the percent difference plots are provided in Table 5.3. Note, all data was obtained over multiple days spanning several months with updates and changes to the system throughout, therefore the consistency found does not depend on single day/run results. 


\begin{tabular}{|ll|lll|ll|}
\hline & & \multicolumn{3}{|c|}{ Energy Efficiency } & \multicolumn{3}{c|}{ Dissociation Rate } \\
Variable & Power Supply & Mean & Std Dev & Skew & Mean & Std Dev \\
Skew
\end{tabular}

Table 5.3: Mean, standard deviation and skew for the percent difference graphs. Note, the percent difference is the percent change in dissociation rate and energy efficiency between two experimental settings (for instance going from 200 SCCM to 300 SCCM) and does not retain any information of the energy efficiency or dissociation of the experiment. 


\subsubsection{Energy Efficiency}

To use DBDs for $\mathrm{CCU}$, the energy costs for $\mathrm{CO}_{2}$ dissociation must be significantly less than the energy released during combustion (the $\mathrm{CO}_{2}$ production process). For example, the combustion of methane has a heating value between $\sim 800-890 \mathrm{~kJ} / \mathrm{mol}(8.3-9.2 \mathrm{eV} / \mathrm{molec})$, therefore if $\mathrm{CO}_{2}$ dissociation is to be viable option for $\mathrm{CCU}$ an energy efficiency of $\sim 35 \%$ would be needed (not including inefficiencies due to electricity production, transport, etc.) [42]. $35 \%$ is a fairly high efficiency for a chemical reaction, but the efficiency limit can be relaxed a number of ways. For instance, if the CCU process produces value-added chemicals (like titanium dioxide, urea, phosgene, etc) and/or the process uses a cheap source of energy (like excess energy produced by power plants in the late evening/early mornings) [31, 148].

The $\mathrm{CO}_{2}$ dissociation efficiencies in VADER were relatively low in all cases with a maximum efficiency of $\sim 2.5 \% \pm 0.5 \%$, for both the TREK and DIDRIV10 energy efficiency results (see Figures 5.2 and 5.3). These low energy efficiencies are well under those needed for a commercial system, however VADER was optimized for finding the variables that affect $\mathrm{CO}_{2}$ dissociation (not efficient dissociation). Other groups have shown that much higher energy efficiencies can be achieved (3\%-10\%), though they still do not reach the efficiencies necessary for commercial implementation $[15,18,58]$.

Comparing the two power supplies (see Figure 5.4) shows that despite similarities in forward power the DIDRIV10 power supply is significantly more efficient at $\mathrm{CO}_{2}$ dissociation than the TREK power supply (TREK 1.25\% versus DIDRIV10 2.5\%).

The main differences between the two power supplies are the driving frequency and voltage. The DIDRIV10 has a significantly lower voltage and a much higher frequency than the TREK power supply. All of the measurements suggest higher voltages lead to higher dissociation rates in VADER (discussed further in the Section 5.4.4). However, the next 

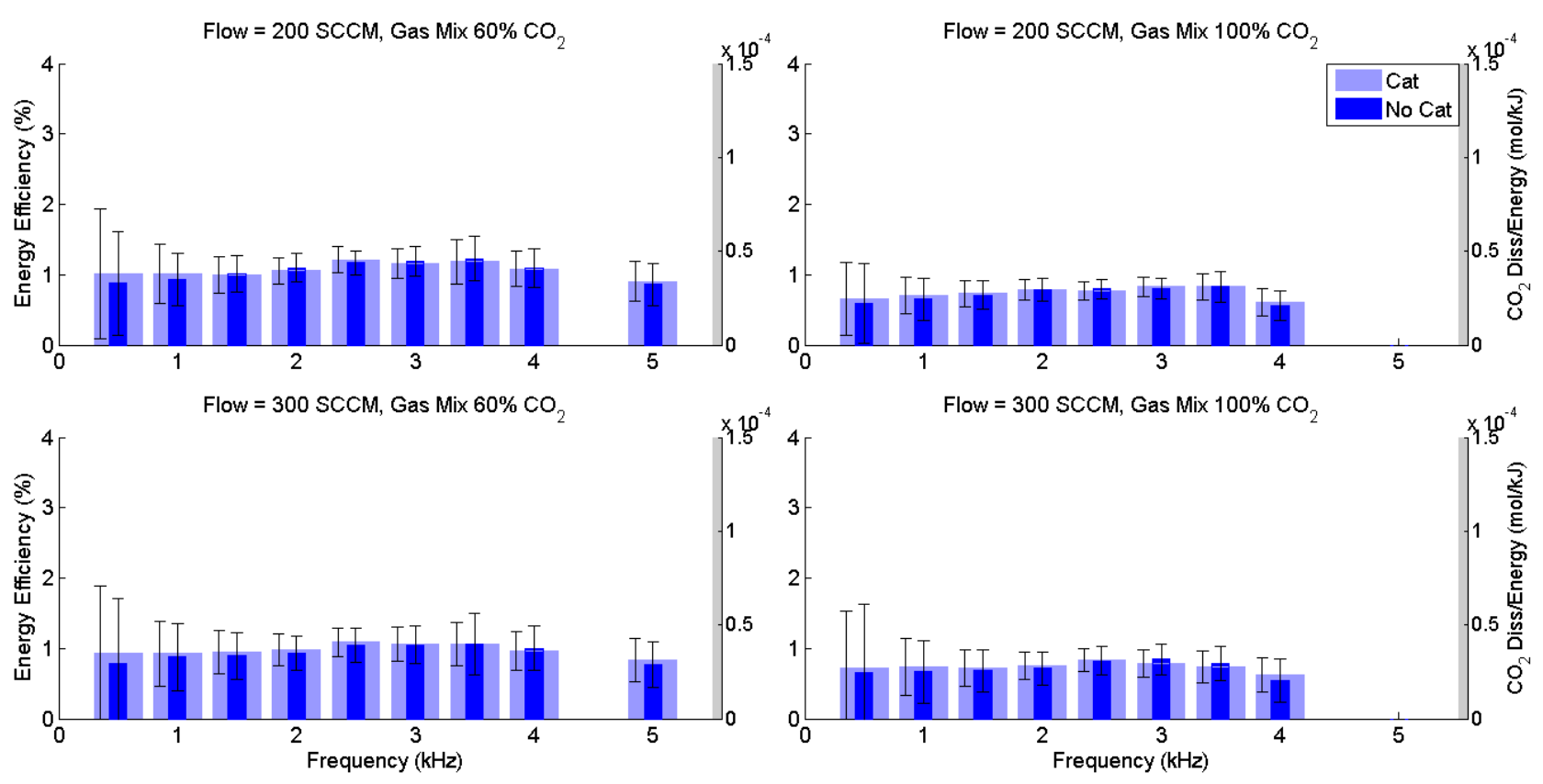

Figure 5.2: Energy efficiency for the TREK experiments.

section shows that despite the much higher voltages used during the TREK power supply experiments, the dissociation rate is much lower than the DIDRIV10 supply experiments, which implies that the difference in voltage only has a minor effect when comparing the two power supplies. Therefore, the frequency must be largely responsible for the higher efficiencies seen in the DIDRIV10 power supply. The plasma chemical model in Section 2.3 shows that driving frequency is important for determining the chemical dynamics of a DBD. Lower frequencies result in longer times within the radical and neutral discharge phases, which are responsible for the reverse $\mathrm{CO}_{2}$ dissociation reactions. Thus, it is important to understand the chemical dynamics of a DBD to obtain the best efficiencies. 

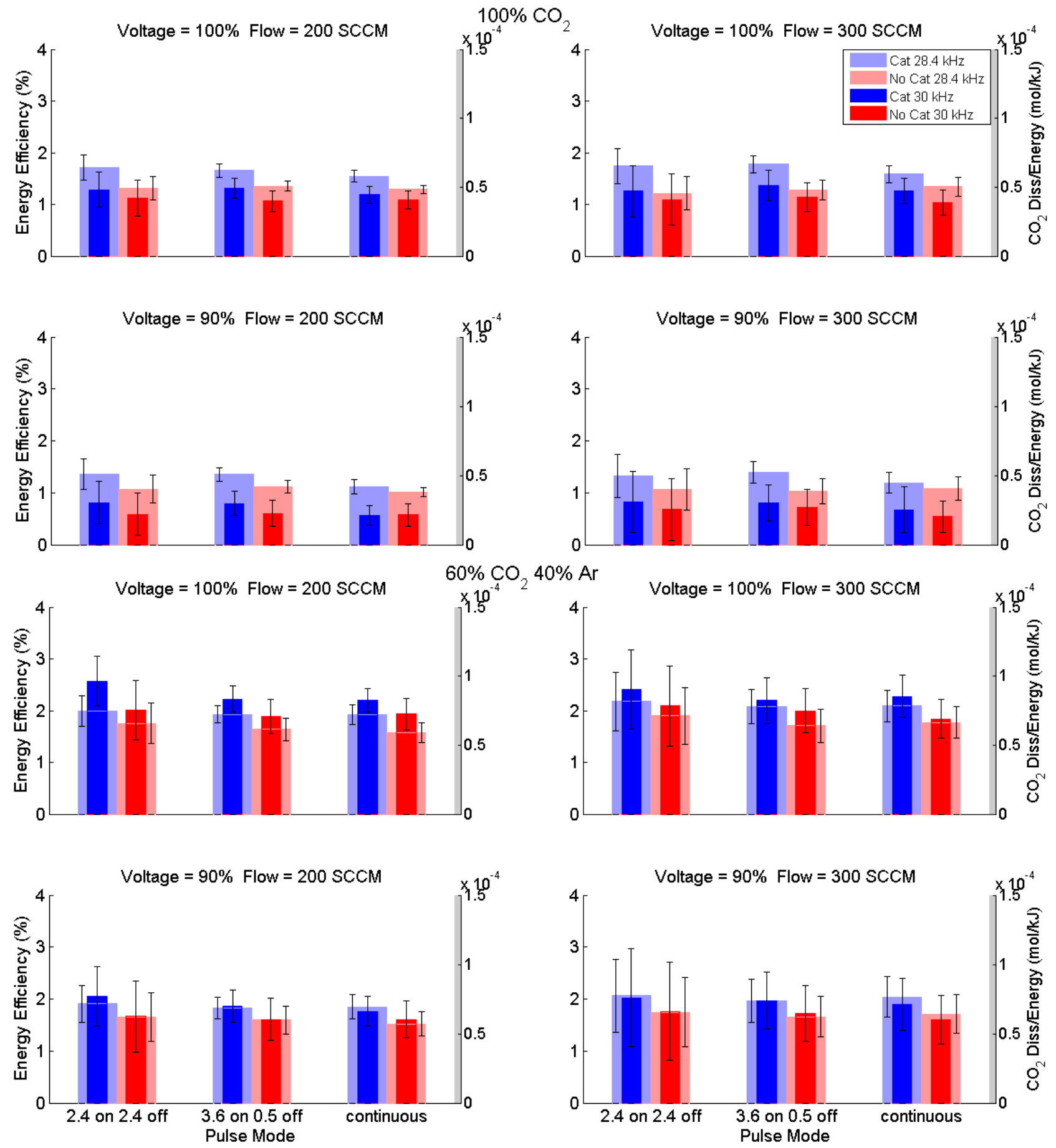

Figure 5.3: Energy efficiency for the DIDRIV10 experiments. The top four graphs were run with $100 \% \mathrm{CO}_{2}$ input gas and the bottom four graphs were with $60 \% \mathrm{CO}_{2}$ and $40 \% \mathrm{Ar}$. 


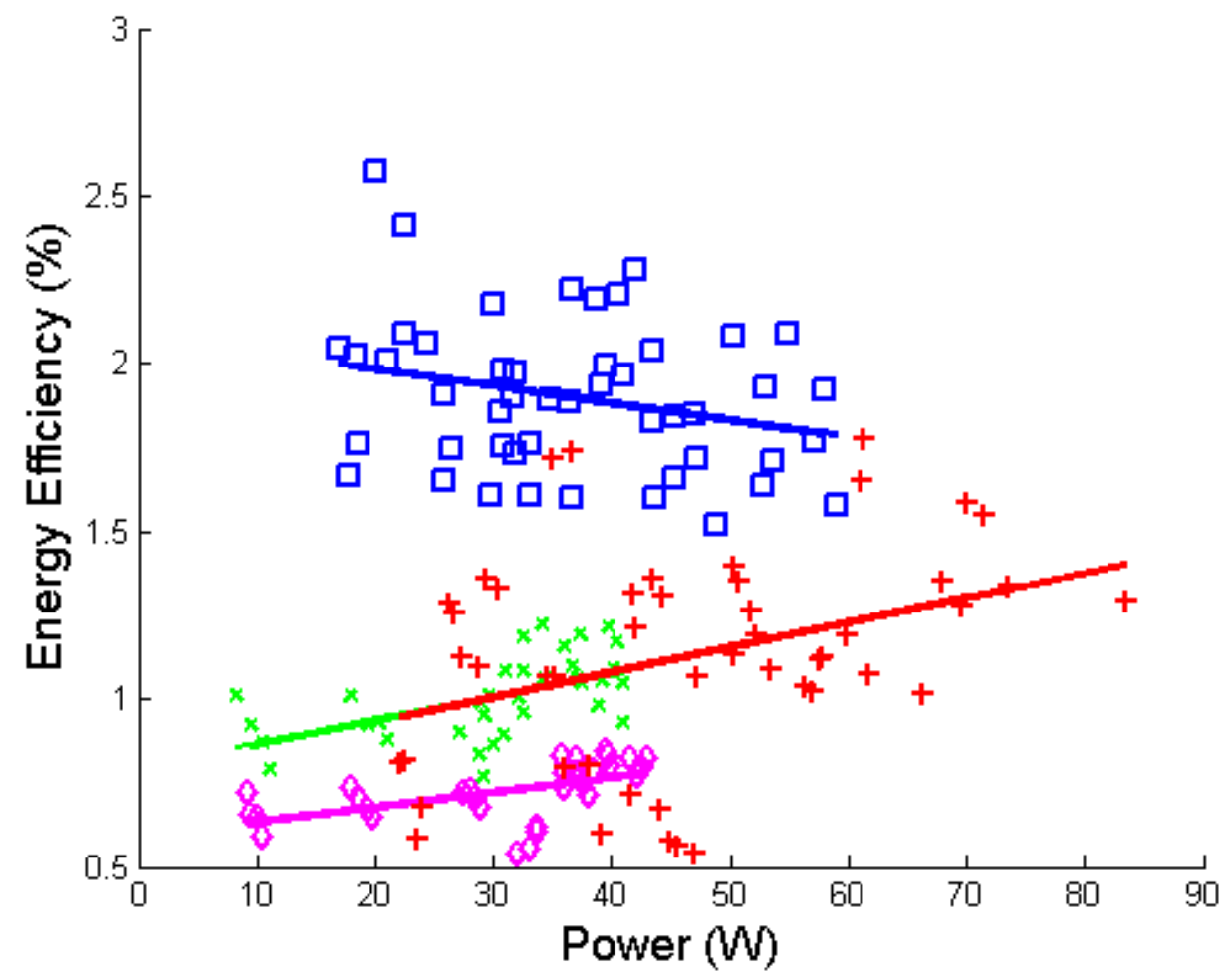

Figure 5.4: Energy efficiency versus power. Blue squares $(\square)$ correspond to experimental runs with $60 \% \mathrm{CO}_{2}$ and $40 \%$ Ar using the DIDRIV10 power supply, red plus signs (+) correspond to $100 \% \mathrm{CO}_{2}$ DIDRIV10 data, green x's (x) correspond to $60 \% \mathrm{CO}_{2}$ TREK data and purple diamonds $(\diamond)$ correspond to $100 \% \mathrm{CO}_{2}$ TREK data.

Figure 5.4 also shows that the addition of argon greatly improves the energy efficiency of $\mathrm{CO}_{2}$ dissociation, doubling the efficiency in some cases. The addition of argon has several effects on the plasma chemistry and the discharge physics, which are discussed in Section 5.4.5.

\subsubsection{Dissociation Rate}

The dissociation rate is important for determining if enough reactions occurred for the system to be worthwhile in a commercial setting. The dissociation rates in VADER using the TREK 
power supply are shown in Figure 5.5 and the rates using the DIDRIV10 power supply are shown in Figure 5.6. The graphs give a maximum reaction rate of $\sim 4 \times 10^{-6} \pm 0.5 \times 10^{-6}$ $\mathrm{mol} / \mathrm{sec}$ during one of the $60 \% \mathrm{CO}_{2} 40 \%$ Ar cases and translates to $\sim 5 \% \mathrm{CO}_{2}$ conversion. These relatively small conversion rates are due to the small amount of power supplied to the DBD (7-85 $\mathrm{W}$ or $\left.2.2-26.5 \mathrm{~W} / \mathrm{cm}^{3}\right)$, which translates to a maximum energy transfer in VADER of $\sim 25.5$ joules $/ \mathrm{cm}^{3}(\sim 6.5 \mathrm{eV} /$ molec $)$.
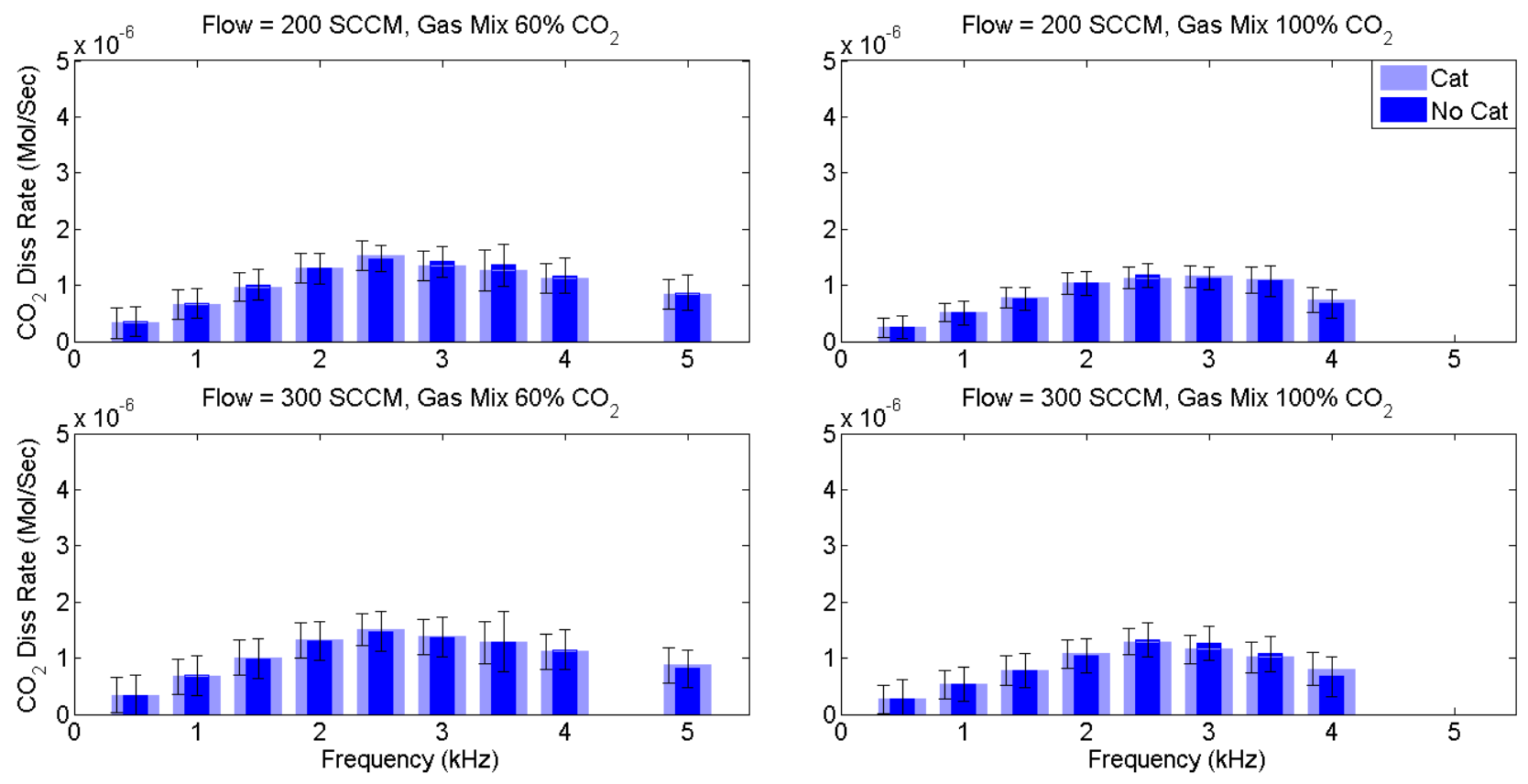

Figure 5.5: TREK dissociation rate

Comparing the dissociation rate with forward power (see Figure 5.7) shows that the dissociation rate increases linearly with increased forward power, which was similarly seen by Paulussen et al. [15]. The forward power in a DBD directly relates to the applied voltage, therefore an increase in forward power increases the streamer density which linearly increases the reaction volume. An increase in the reaction volume means more gas is being reacted and reduces the time during which a gas cools between streamers. While not seen in these 

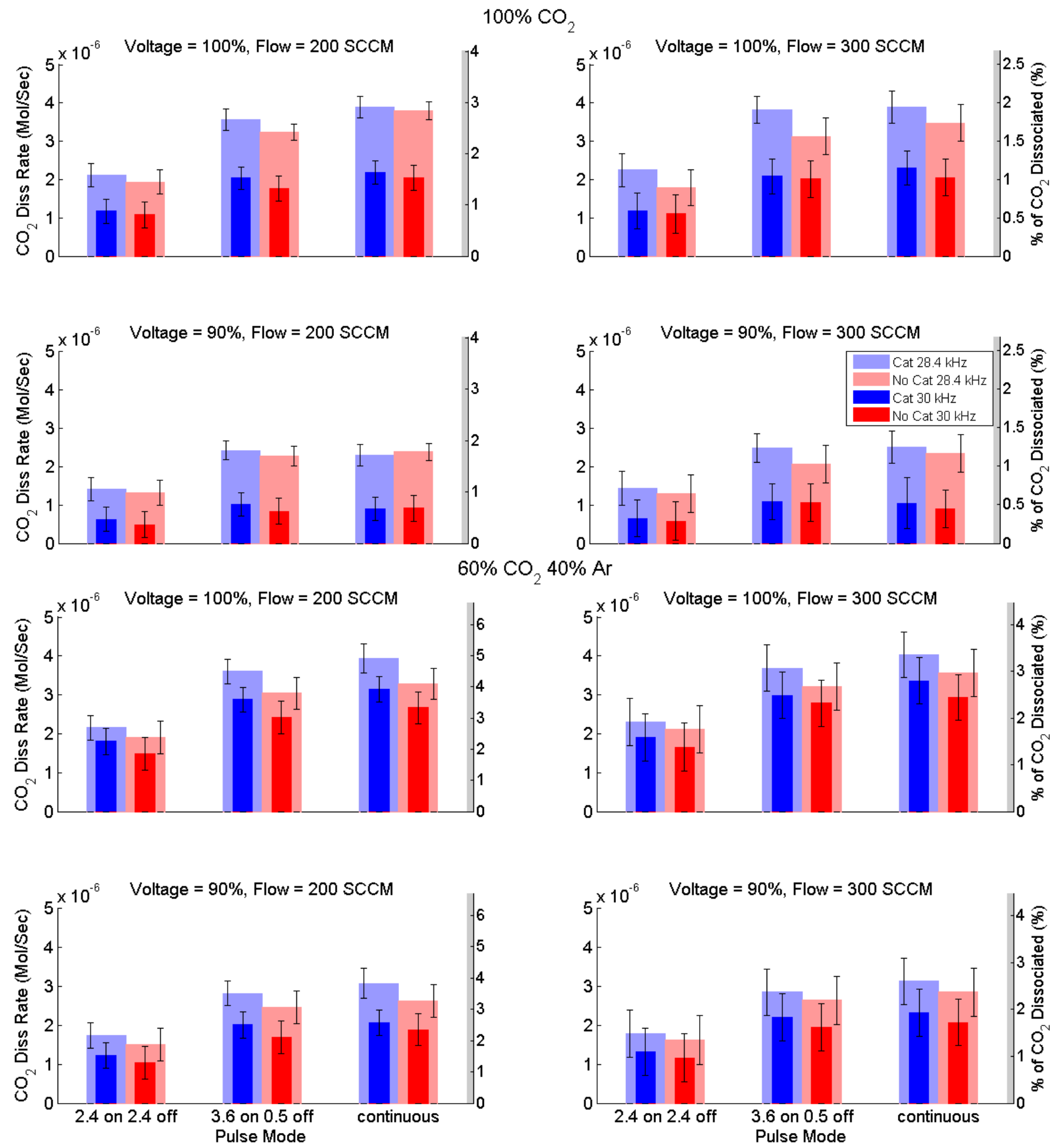

Figure 5.6: DIDRIV10 $\mathrm{CO}_{2}$ dissociation rate - The top four cases were run with $100 \%$ $\mathrm{CO}_{2}$ input gas and the bottom four cases were with $60 \% \mathrm{CO}_{2}$ and $40 \% \mathrm{Ar}$. 
results, Wang et al. showed that there is a point of saturation where the relationship between dissociation rate and voltage eventually plateaus and only small gains in dissociation rate are attained [18].

Figure 5.7 also shows that the DIDRIV10 power supply dissociates considerably more than the TREK power supply and the introduction of argon into the gas significantly improves $\mathrm{CO}_{2}$ dissociation rates in a DBD, despite similar forward powers and a reduction in $\mathrm{CO}_{2}$ concentrations. The difference between the power supplies is likely due to their frequency difference, as discussed in Section 5.4.1. The main effects of adding argon into the feed gas are a reduction in the plasma breakdown voltage and an increase in $\mathrm{CO}_{2}^{+}$populations due to charge exchange with argon ions (which leads to more dissociation), which is discussed further in Section 5.4.5). These increases in dissociation rate are accompanied by an increase in the slope of the lines (noted in the figure caption), which implies the effects of frequency and gas composition have a non-linear effect on the dissociation rate.

\subsubsection{Gas Flow Rate}

The gas flow rate is representative of the gas residence time within the plasma. An increase in the residence time gives the plasma more time to react a volume of gas, meaning a larger percent of the input gas is reacted as well as more time for product gases to be reacted. As discussed in Section 2.3, an increase in the density of $\mathrm{CO}_{2}$ dissociation products reduces the energy efficiency in a DBD. Therefore, it was expected that a higher flow rate leads to a higher energy efficiency, but a lower percent dissociation.

The two power supplies had very different results. Figure 5.8 shows that when using the TREK power supply experiments with $100 \% \mathrm{CO}_{2}$, changing the gas flow rate has no effect on the energy efficiency nor the dissociation rate. However, when using $60 \% \mathrm{CO}_{2}$ and 


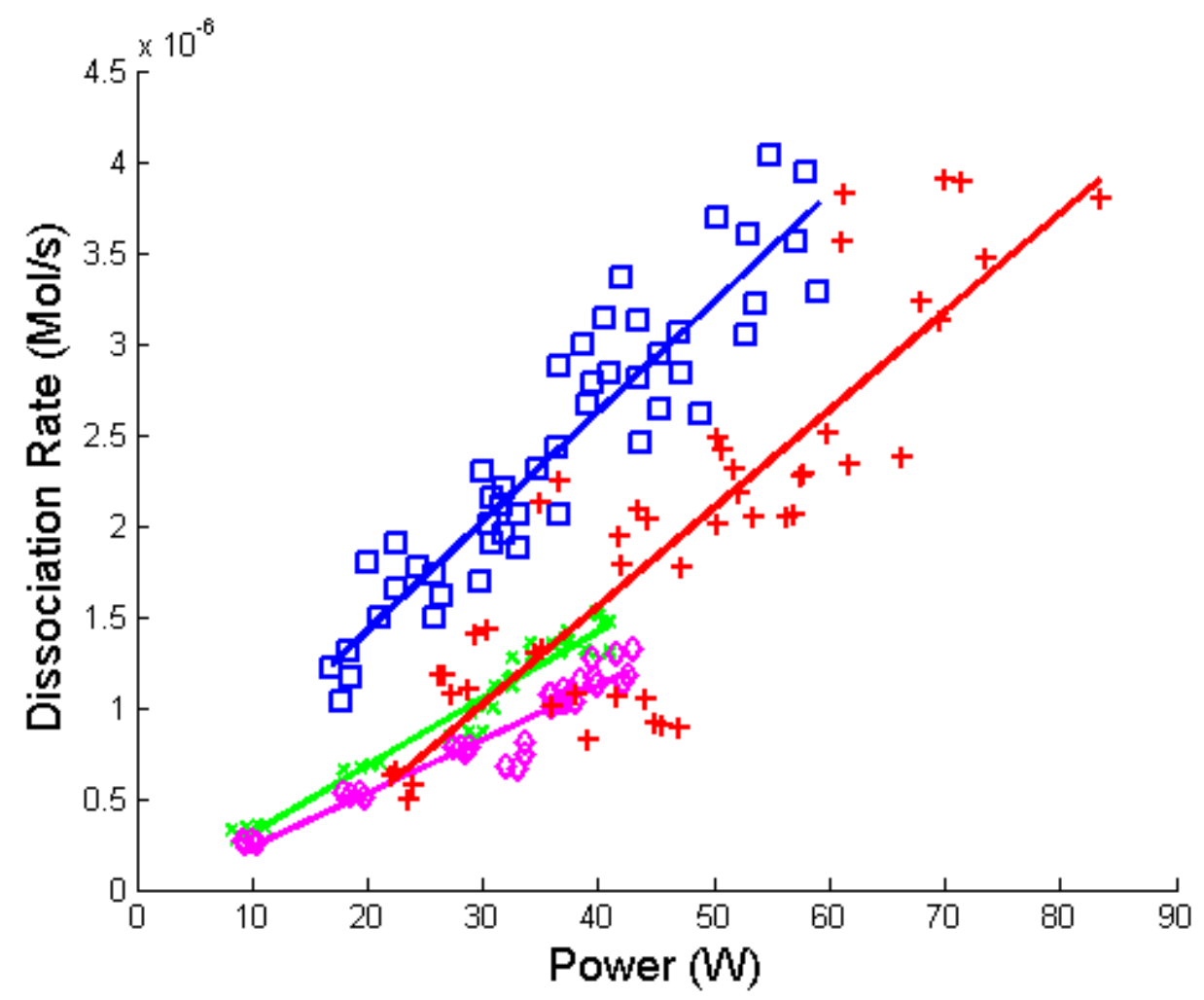

Figure 5.7: $\mathrm{CO}_{2}$ dissociation rate versus power. Blue squares ( $\square$ ) correspond to experimental runs with $60 \% \mathrm{CO}_{2}$ and $40 \%$ Ar using the DIDRIV10 power supply (slope $=6.02 \times 10^{-8}$ $\mathrm{mol} / \mathrm{sW}, \mathrm{R}^{2}=0.865$ ), red plus signs $(+)$ correspond to $100 \% \mathrm{CO}_{2}$ DIDRIV10 data (slope $\left.=5.39 \times 10^{-8} \mathrm{~mol} / \mathrm{sW}, \mathrm{R}^{2}=0.746\right)$, green $\mathrm{x}$ 's $(\mathrm{x})$ correspond to $60 \% \mathrm{CO}_{2}$ TREK data (slope $\left.=3.71 \times 10^{-8} \mathrm{~mol} / \mathrm{sW}, \mathrm{R}^{2}=0.942\right)$ and purple diamonds $(\diamond)$ correspond to $100 \%$ $\mathrm{CO}_{2}$ TREK data (slope $\left.=2.96 \times 10^{-8} \mathrm{~mol} / \mathrm{sW}, \mathrm{R}^{2}=.923\right)$.

$40 \%$ argon, the results show that by increasing the flow rate the energy efficiency decreases by $10 \%$, while the dissociation rates remain unchanged. However, the DIDRIV10 graphs in Figure 5.8 show higher flow rates correlate with a higher energy efficiency and dissociation rate. It is proposed that this discrepancy in efficiency and dissociation rate trends is due to the differences in driving frequencies of the two power supplies. The DIDRIV10 has a frequency range of $28.4-30 \mathrm{kHz}$, which is very fast in comparison to the gas residence time (0.644 s at $300 \mathrm{SCCM}$ and $0.97 \mathrm{~s}$ at $200 \mathrm{SCCM}$ ) and corresponds to the gas going through 

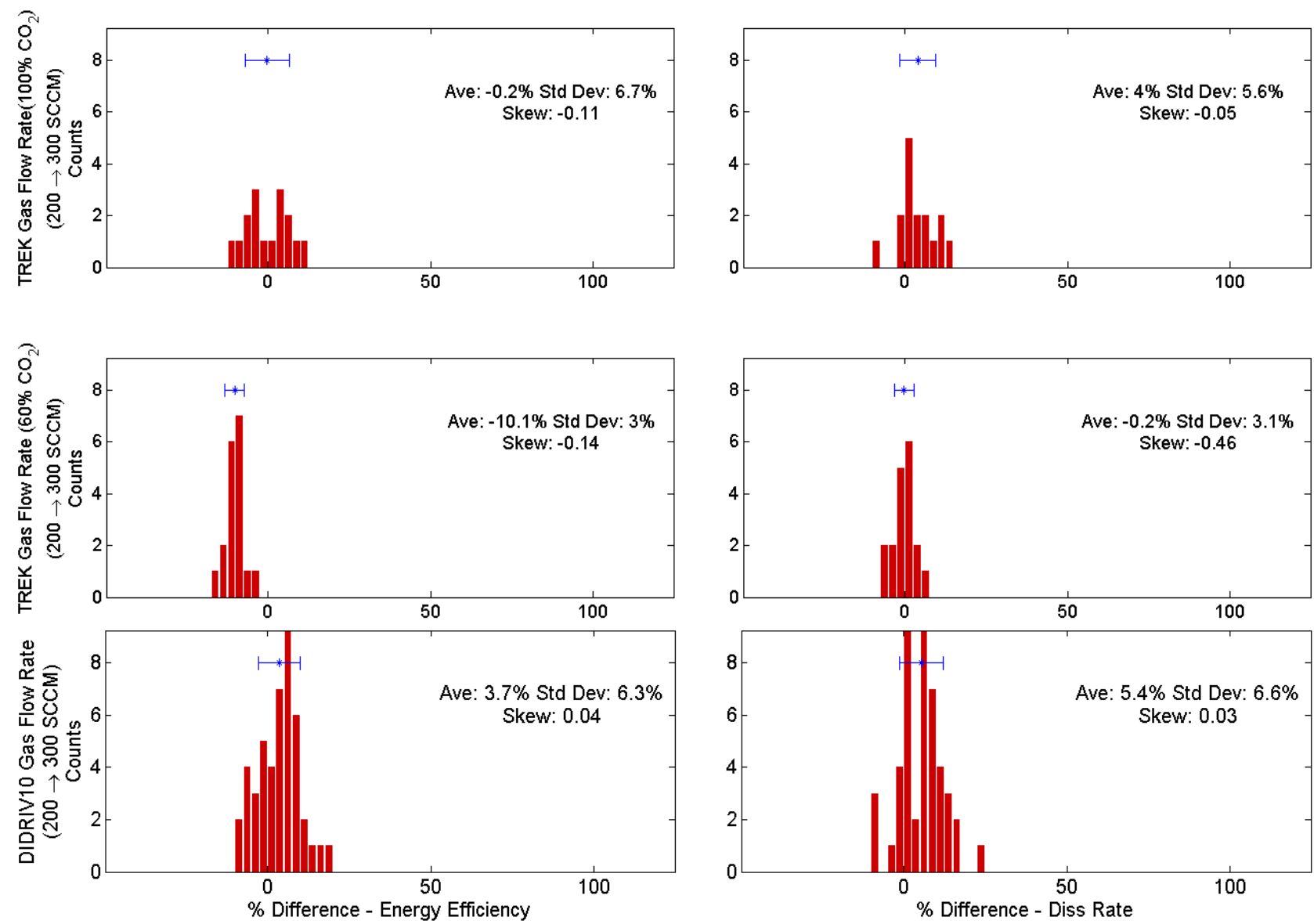

Figure 5.8: Percent difference (change) in energy efficiency and dissociation rate between gas flow rate settings in VADER.

tens of thousands of discharge cycles before leaving the reaction box. These large numbers of discharge cycles allow for product gases to quickly build up in the system and for molecular energies (vibrational, electronic, etc) to accumulate over multiple discharge cycles within each streamer. By increasing the flow rate, a lower density of product gas is available for the reverse reaction (because the gas experiences fewer discharge cycles) and there is less energy accumulation, thus increasing the energy efficiency and dissociation rate. However, the increase in flow rate does adversely affect the percent of input gas dissociated. In the case of the TREK power supply, the driving frequencies are slow $(0.5-5 \mathrm{kHz})$, thus product 
densities stay small and there is limited energy accumulation. Therefore, the cold plasma with small product densities allows the plasma to stay in a regime where the re-use of excited species in the discharge is more beneficial to $\mathrm{CO}_{2}$ dissociation than the effects of product buildup and heating.

\subsubsection{Voltage}

The characteristics of a streamer are invariant with respect to the driving voltage, therefore the voltage determines the number and density of streamers in a DBD [74, 149]. The invariance is due to the short lifetime of a streamer (10s of nanoseconds) in comparison to the relatively slow changes in voltage even at the higher frequencies used in VADER $(\sim 33$ $\mu$ s for one cycle at $30 \mathrm{kHz}$ ) [150]. The density of streamers affects the amount of time a volume of gas is reacted within streamers (as opposed to between streamers), which changes the length of time a gas is highly excited and is within the neutral phase. An excited gas in the neutral phase strongly excites reverse reactions (described in Section 2.3.2), therefore a dense streamer population reduces reverse reaction rates and improves energy efficiency. This reduction in reverse reactions explains the large percent increases in energy efficiency and dissociation rate as voltage is increased in the DIDRIV10 experiments, see Figure 5.9. The reason for the large variance in the percent difference data is because voltage, gas composition and frequency are interrelated. The reasons are further discussed in Section 5.4.6.

These results run counter to results by Wang et al., Zheng et al. and Paulussen et al., who show that with increased voltage there is an increase in dissociation rate and a decrease in energy efficiency $[15,18,58]$. However, both Wang et al. and Zheng et al. used considerably lower frequencies than the DIDRIV10 power supply (1-4 kHz versus $28.4-30 \mathrm{kHz}$ ). These smaller frequencies mean the reacted gas spends considerably more time within the neutral 

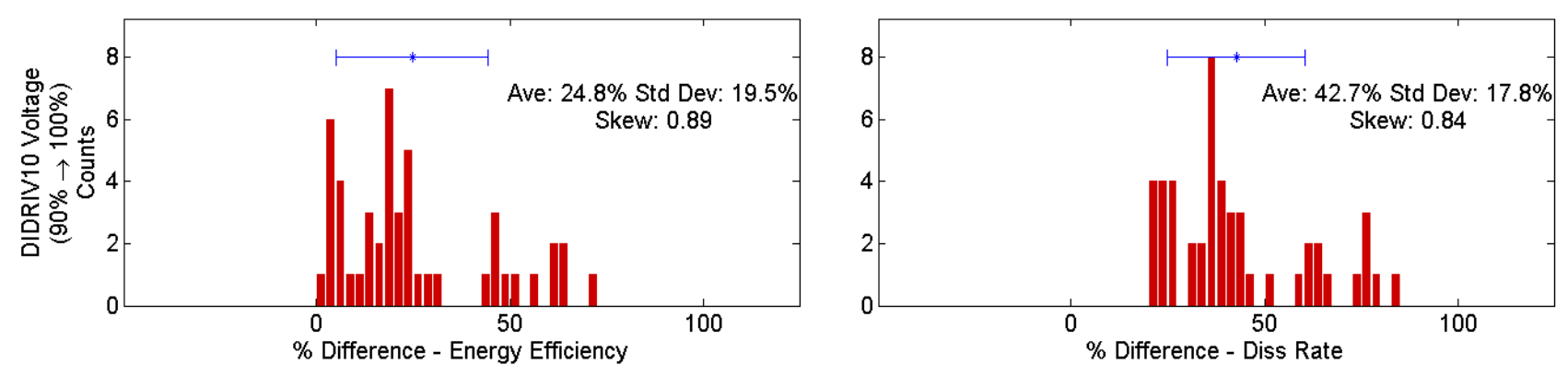

Figure 5.9: Percent difference (change) in energy efficiency and dissociation rate between power supply voltage settings in VADER.

phase, therefore increasing the voltage increases the volume and density of product gases that end up spending considerable time within the neutral phase. More time within the neutral phase leads to higher dissociation rates and lower energy efficiencies as seen in their work. Paulussen et al. used a similar frequency to those used in VADER, but used much higher voltages (15 kVp-p versus 3-4 kVp-p), a shorter air gap (reduces breakdown voltage) and had a much larger percent conversion (concentrations of product gases) than VADER ( 12-15\% versus $\sim 5 \%$ with a 200 SCCM flow rate). The pulse mode results (Section 5.4.8) show that the effects of product gas concentrations were small for all of the VADER results. Therefore, it is expected that there is a tipping point, where the inefficiencies due to reverse reactions by product gases overcomes the efficiency gains due to other effects, like increased voltages. Thus, there is a peak voltage at which energy efficiency is a maximum, which will often not correlate with the dissociation rate. It is also expected that higher voltages, smaller discharge volumes, and lower breakdown voltages eventually lead to streamer saturation, at which point increasing the voltage no longer increases the density of streamers and instead increases the temperature and density of the plasma, as seen by Liu et al. [151]. As discussed, any change in temperature and density drastically changes the chemical dynamics of a plasma, and the effects of which were beyond the scope of this work. 


\subsubsection{Gas Composition}

The results of the plasma chemical model in Section 2.3 suggest that the addition of argon into the plasma improves the efficiency of $\mathrm{CO}_{2}$ dissociation in a DBD because argon preferentially goes through charge exchange with $\mathrm{CO}_{2}$. It is proposed that this charge exchange process allows $\mathrm{CO}_{2}$ recombination $\left(\mathrm{CO}_{2}^{+}+e \rightarrow C O+O\right)$ to occur at a higher frequency. In addition to changing the plasma chemistry, argon reduces the breakdown voltage of a DBD. This reduction in breakdown voltage is due to the large electron binding energies of argon, which make it difficult for free electrons to have inelastic collisions with argon [152]. The combination of reduced inelastic collision rates with the low recombination rate of argon (discussed in Section 2.3) effectively increase the mean free path of electrons in the plasma, which gives electrons a longer time to accelerate within the external electric fields. This increase in mean free path is balanced with the lower voltages needed for breakdown, thus the electron temperatures and densities are invariant to the change in breakdown voltage, as seen by Zhang et al. [153]. A reduction in breakdown voltage is equivalent to increasing the applied voltage, because in both cases you increase the breakdown ratio $\left(\frac{V_{\max }}{V_{B r}}\right)$, see Figure 5.15. Considering increased voltages in VADER led to higher dissociation rates and efficiencies (see Section 5.4.4), and the plasma chemistry suggests that argon should help with dissociation, the addition of argon in VADER was expected to increase the energy efficiency and dissociation rate.

The experimental results for both power supplies show that the energy efficiency and dissociation rate increase drastically with the addition of argon into the feed gas. The TREK experiments (Figure 5.10) showed an average percent difference in energy efficiency and dissociation rate of $34.4 \%$ and $25 \%$, respectively, when going from $100 \% \mathrm{CO}_{2}$ cases to the $60 \% \mathrm{CO}_{2}$ cases. The DIDRIV10 experiments had a percent difference of $53.4 \%$ and $31.1 \%$, 

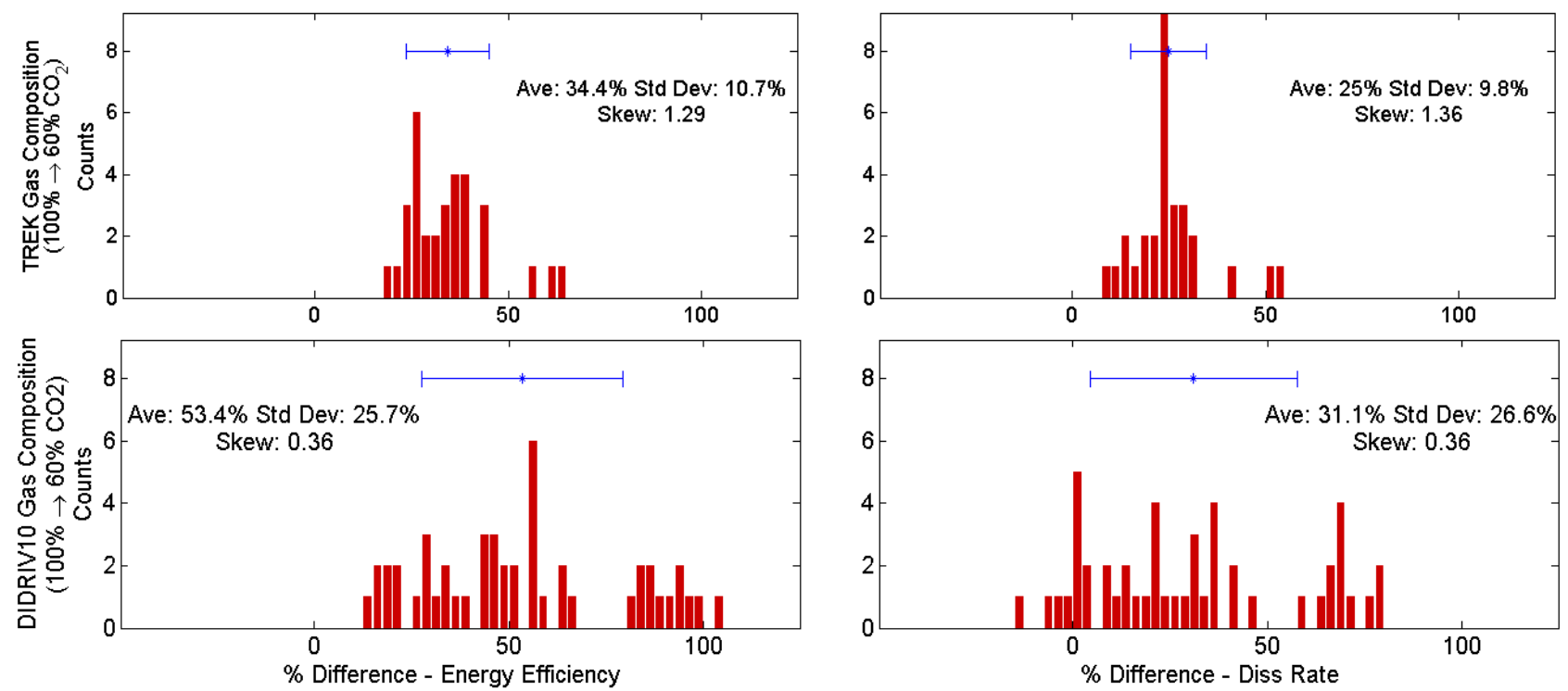

Figure 5.10: Percent difference (change) in energy efficiency and dissociation rate when using $100 \% \mathrm{CO}_{2}$ and $60 \% \mathrm{CO}_{2}$ and $40 \%$ argon in VADER.

respectively. Since the streamer plasma properties in this plasma regime are invariant to gas composition and forward power, the change in chemical kinetics and increase in the discharge volume must be responsible for the increase in dissociation rate and energy efficiency.

These increases in efficiency and dissociation rate with the addition of argon occur despite a $10 \%$ decrease in the maximum voltage and a $23.7 \%$ decrease in forward power, see Figure 5.11. The reduced voltage is due to the lower breakdown voltages caused by the addition of argon and is similar to what occurred when different dielectric materials were used in VADER (Section 5.1). The decrease in power is due to lower voltages, which reduce power loss to internal resistance, corona discharge across the power cables, and dielectric heating. As this change in power directly correlates to $P=V^{2} / R$, it is suggested that internal resistance is a significant loss mechanism in a DBD.

The reason for the large variance in the DIDRIV10 percent difference data is because voltage, gas composition and frequency are interrelated. The reasons are further discussed 
in Section 5.4.6.
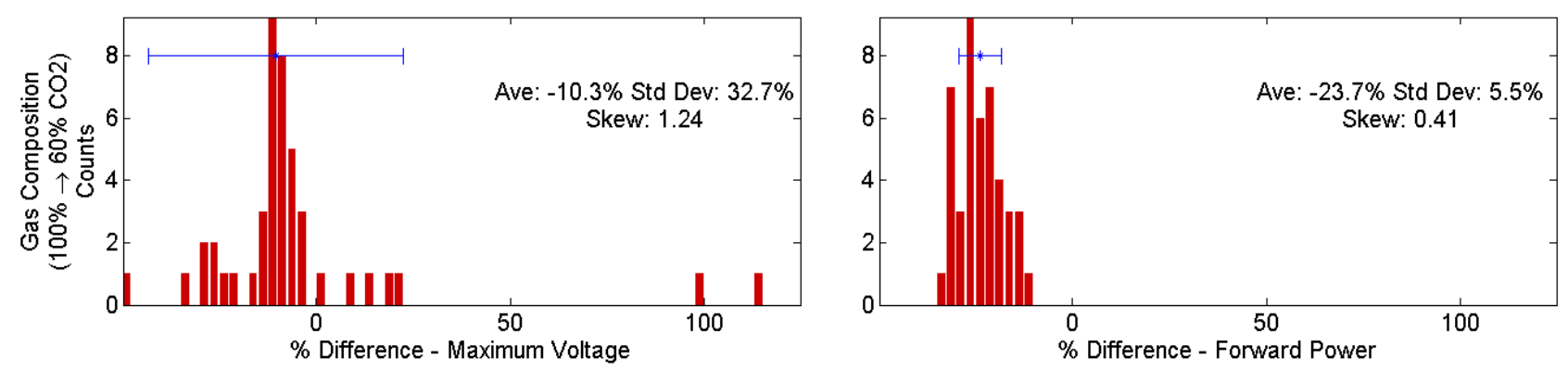

Figure 5.11: Percent Difference in Forward Power between different Gas Compositions for the DIDRIV10 Power Supply. The distribution shows that by only changing the gas composition the forward power of the DBD decreases.

\subsubsection{Driving Frequency}

As discussed in Section 5.4.1, the driving frequency is a major factor when determining the efficiency of chemical reactions in a DBD. The frequency controls the time the plasma is within each of the three discharge phases, which determines the reaction kinetics of the plasma. The effects of frequency in VADER can be seen by plotting the dissociation efficiency versus frequency (see Figure 5.12), which show that frequencies near $30 \mathrm{kHz}$ are significantly more efficient than frequencies in the range of $1-5 \mathrm{kHz}$.

Figure 5.13 shows the percent difference (change) in energy efficiency and dissociation rate when the frequency is reduced from $30 \mathrm{kHz}$ to $28.4 \mathrm{kHz}$ when using the DIDRIV10 power supply. Both plots are quite broad and do not demonstrate the effects of frequency on the energy efficiency and dissociation. However, further analysis shows that these percent difference distributions are highly dependent on the voltage and gas composition' as seen by the similarly broadened percent difference distributions for voltage and gas composition-see Figures 5.9 and 5.10, respectively. Splitting the frequency percent difference graphs up by the 


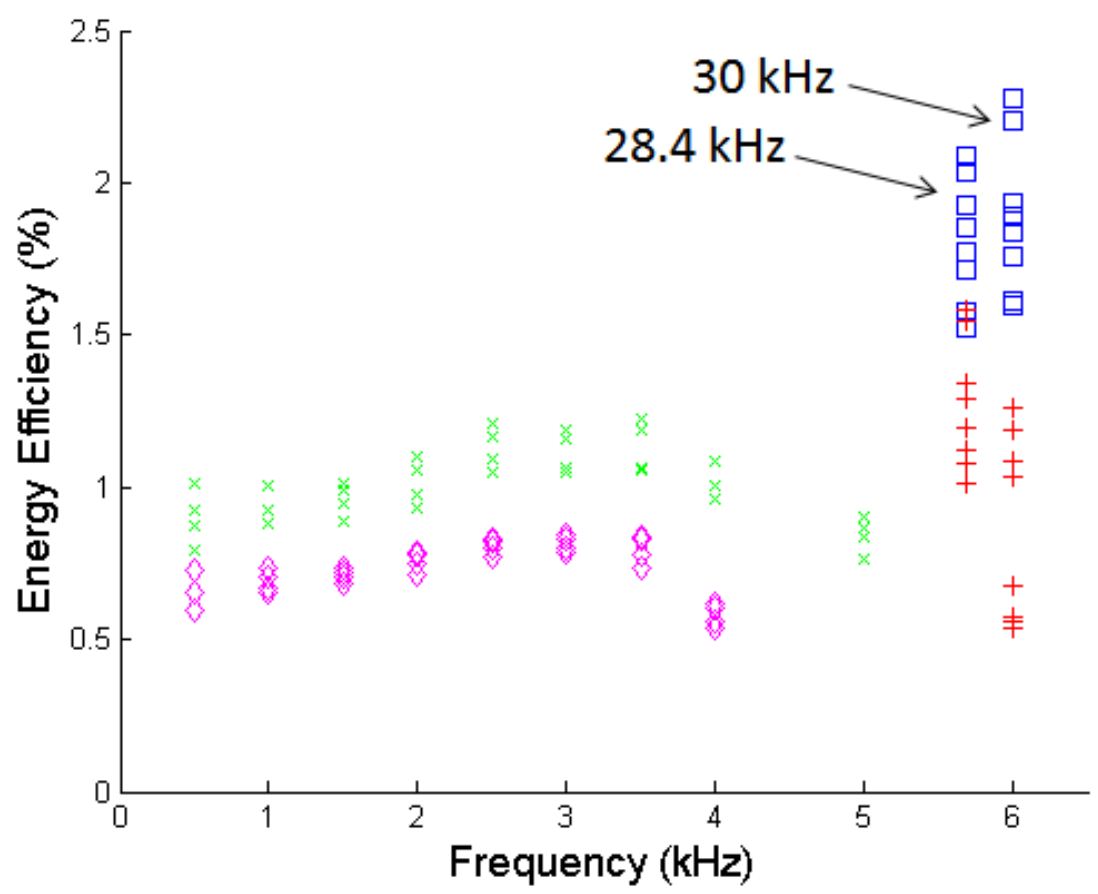

Figure 5.12: Energy efficiency versus frequency. Blue squares $(\square)$ correspond to experimental runs with $60 \% \mathrm{CO}_{2}$ and $40 \%$ Ar using the DIDRIV10 power supply, red plus signs $(+)$ correspond to $100 \% \mathrm{CO}_{2}$ DIDRIV10 data, green x's (x) correspond to $60 \% \mathrm{CO}_{2}$ TREK data and purple diamonds $(\diamond)$ correspond to $100 \% \mathrm{CO}_{2}$ TREK data. The drop in energy efficiencies between $3-5 \mathrm{kHz}$, are due to the voltage limits of the TREK power supply, discussed in Section 3.1.2. Each data point has an error of $<0.5 \%$, except at .5 $\mathrm{kHz}$ which has error $<1 \%$.

different voltage and gas compositions gives considerably more consistent data-see Figure 5.14. By comparing the distributions with the same voltage and differing gas compositions (plots A and B; and plots $\mathrm{C}$ and D), the gas composition is seen to have a major effect on the percent difference values for changes in frequency. For example, in plot A the percent difference is positive $(22 \% \pm 6.9 \%)$, which implies that when the gas is $100 \% \mathrm{CO}_{2}$ and the power supply is at max voltage dissociation is more efficient at lower frequencies $(28.4 \mathrm{kHz}$ as opposed to $30 \mathrm{kHz}$ ). However, in plot $\mathrm{B}$, where the gas is $60 \% \mathrm{CO}_{2}$ and $40 \%$ argon, the percent difference is negative $(-12.9 \% \pm 6.1 \%)$ meaning higher frequencies are more efficient. 
This change in energy efficiency with gas composition is similarly seen when comparing plots $\mathrm{C}$ and D. In plot $\mathrm{C}$ with $100 \% \mathrm{CO}_{2}$ and $90 \%$ voltage lower frequencies are more efficient and at $60 \% \mathrm{CO}_{2}$ and $90 \%$ voltage the efficiency doesn't change with frequency. A similar trend is found when the same gas compositions and differing voltages are compared (plots A and $\mathrm{C}$; and plots $\mathrm{B}$ and $\mathrm{D}$ ). In the case of differing voltages, it shows that $\mathrm{CO}_{2}$ dissociation is more efficient at higher frequencies when higher voltages are applied. A similar effect was seen by Paulussen et al. and $\mathrm{Li}$ et al., where they show that $\mathrm{CO}_{2}$ conversion rates are both frequency and power (which relates to voltage) dependent $[15,136]$.
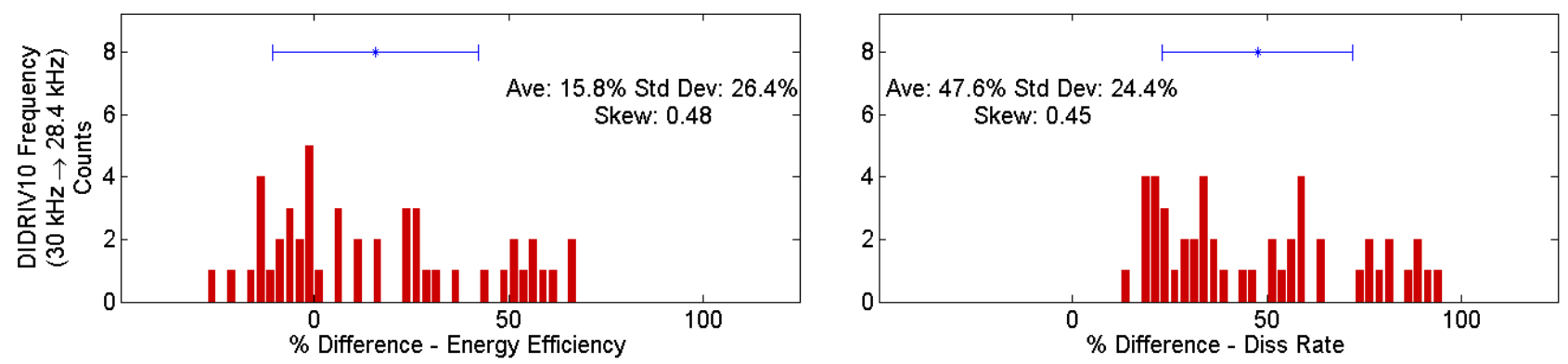

Figure 5.13: Percent difference (change) in energy efficiency and dissociation rate between frequency settings in VADER.

The changes in $\mathrm{CO}_{2}$ dissociation energy efficiency with frequency imply that there is a resonance between the driving frequency and the plasma chemistry within a DBD. It is proposed that this occurs due to a predator-prey relationship between the different plasma reactions. If the frequency is too slow, the gas spends additional time within the neutral phase going through the reverse reactions. If the frequency is too high, the gas doesn't have time to relax and form the final products. This short relaxation time also leads to an increase in the vibrational energy of molecules (it would explain why DBD excimer lamps work best at higher frequencies, $10 \mathrm{~s}-100 \mathrm{~s}$ of $\mathrm{kHz})[149,154]$. This build up in energy causes more $\mathrm{CO}_{2}$ breakdown, but also encourages the breakdown of the final products and the 

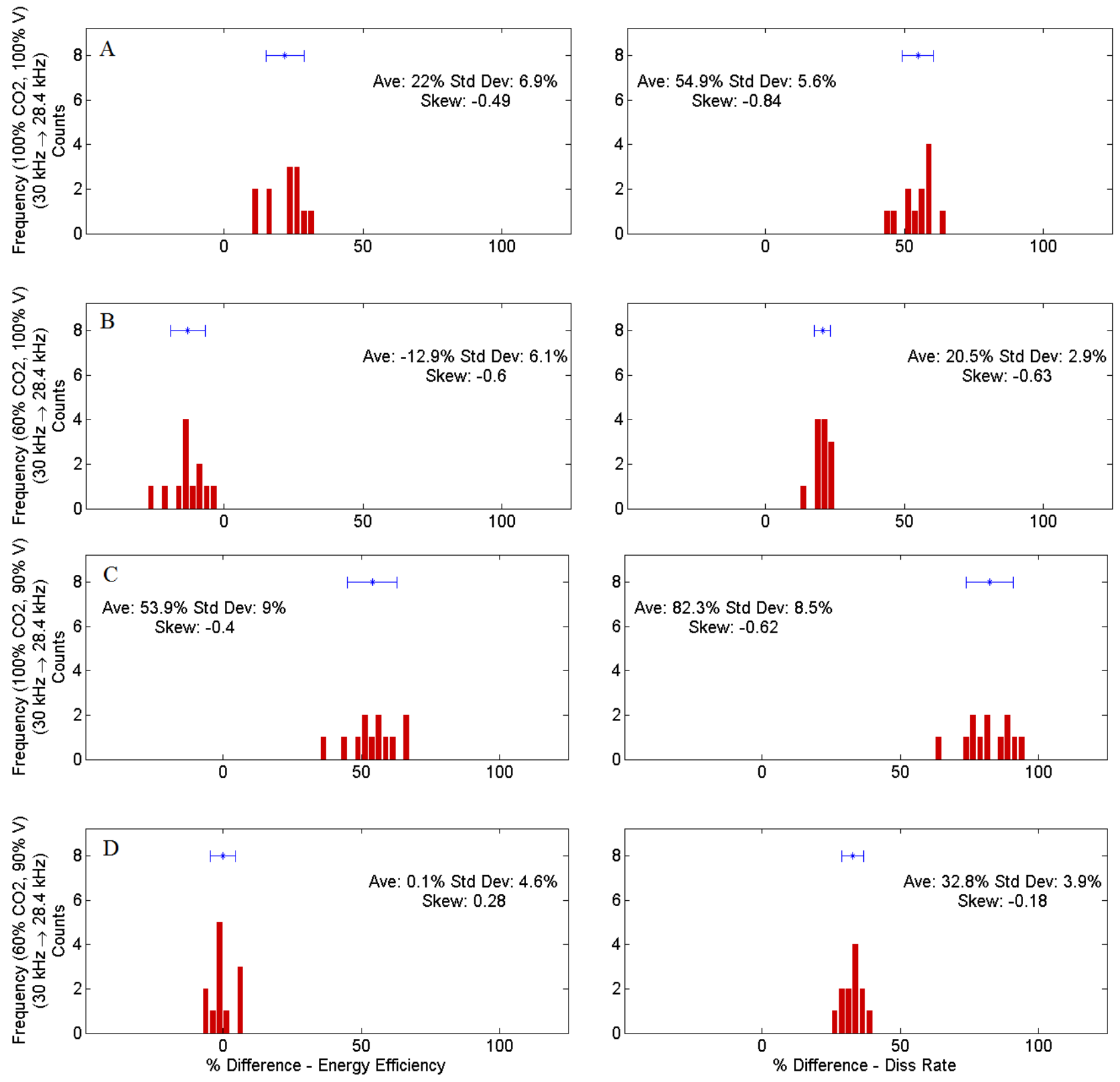

Figure 5.14: Percent difference (change) in energy efficiency and dissociation rate between frequency settings when using the DIDRIV10 power supply. The percent differences are separated by gas composition and power supply voltage. 
production of a long lived free radical population which increases the afterglow reaction rates, like $\mathrm{O}+\mathrm{CO} \rightarrow \mathrm{CO}_{2}$. This means there needs to be a balance between relaxation times (time for final products to form) and the discharge times (time where $\mathrm{CO}_{2}$ is getting dissociated).

These experimental results also show that two major factors in determining the resonant frequency are the applied voltage and the gas composition. Increasing the power supply voltage and lowering the breakdown voltage (due to changes in the gas composition) both

leads to an increase in the breakdown ratio $\left(\frac{V_{\max }}{V_{B r}}\right)$, which correlates with an increase in the number of streamers and extends the breakdown times (see Figure 5.15). Both of these effects lead to the gas spending more time within the discharge and less time relaxing. The experiments show that this increase in discharge time leads to a higher frequency plasma being more efficient at dissociation. A higher frequency reduces both the relaxation time and the discharge time, which makes the discharge time closer to what it was before the voltage was increased (or breakdown voltage was reduced). Therefore, it is expected that there is also some form of resonance between the breakdown time and $\mathrm{CO}_{2}$ dissociation.

\subsubsection{Catalyst}

Wang et al. showed that the high voltage surface material has significant effects on dissociation rate and efficiency. However, the results of Wang et al. are complicated by significant sputtering of the electrode surfaces. The VADER experiments showed no sign of sputtering or material reactions. The catalyst tested in VADER was the photocatalyst $\mathrm{P} 25 \mathrm{TiO}_{2}$, see Section 2.1.3 for details. The expectation was that if the plasma imparts energy above the activation energy of the photocatalyst, then the photocatalyst would improve both the energy efficiency and the dissociation rate of VADER. As the surface area of $\mathrm{TiO}_{2}$ in $\mathrm{VADER}$ 


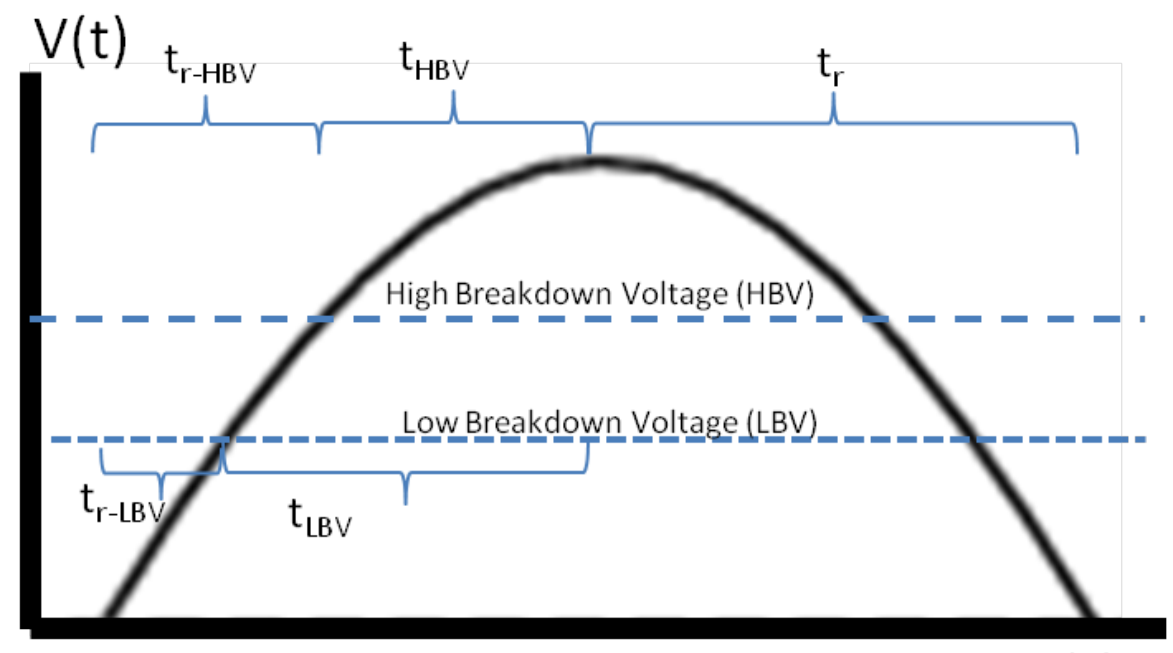

Time (t)

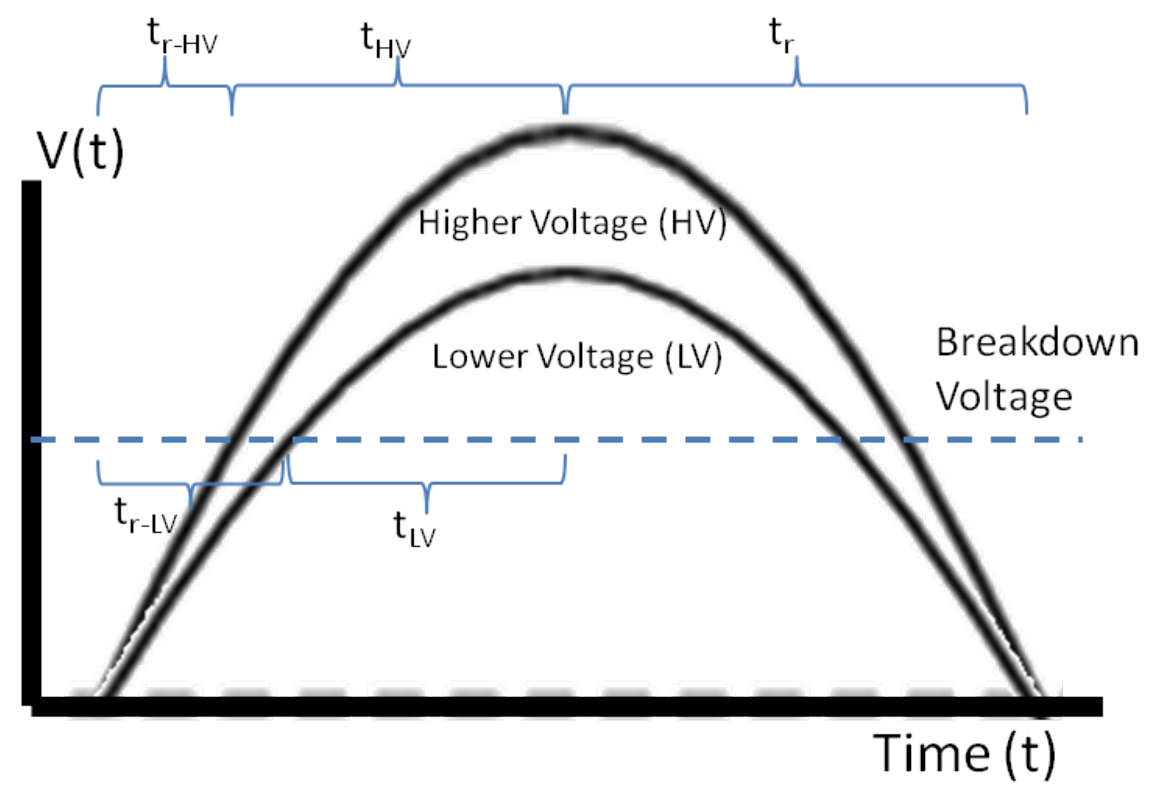

Figure 5.15: Effects of lower breakdown voltages and higher applied voltages in a DBD - $t_{r}$ and $t_{r-x}$ are the relaxation times (time between discharges), and $t_{H B V}, t_{L B V}, t_{H V}$ and $t_{L V}$ are the times when streamer discharges can occur (breakdown time). 
was relatively small, the improvement in dissociation was expected to be small.
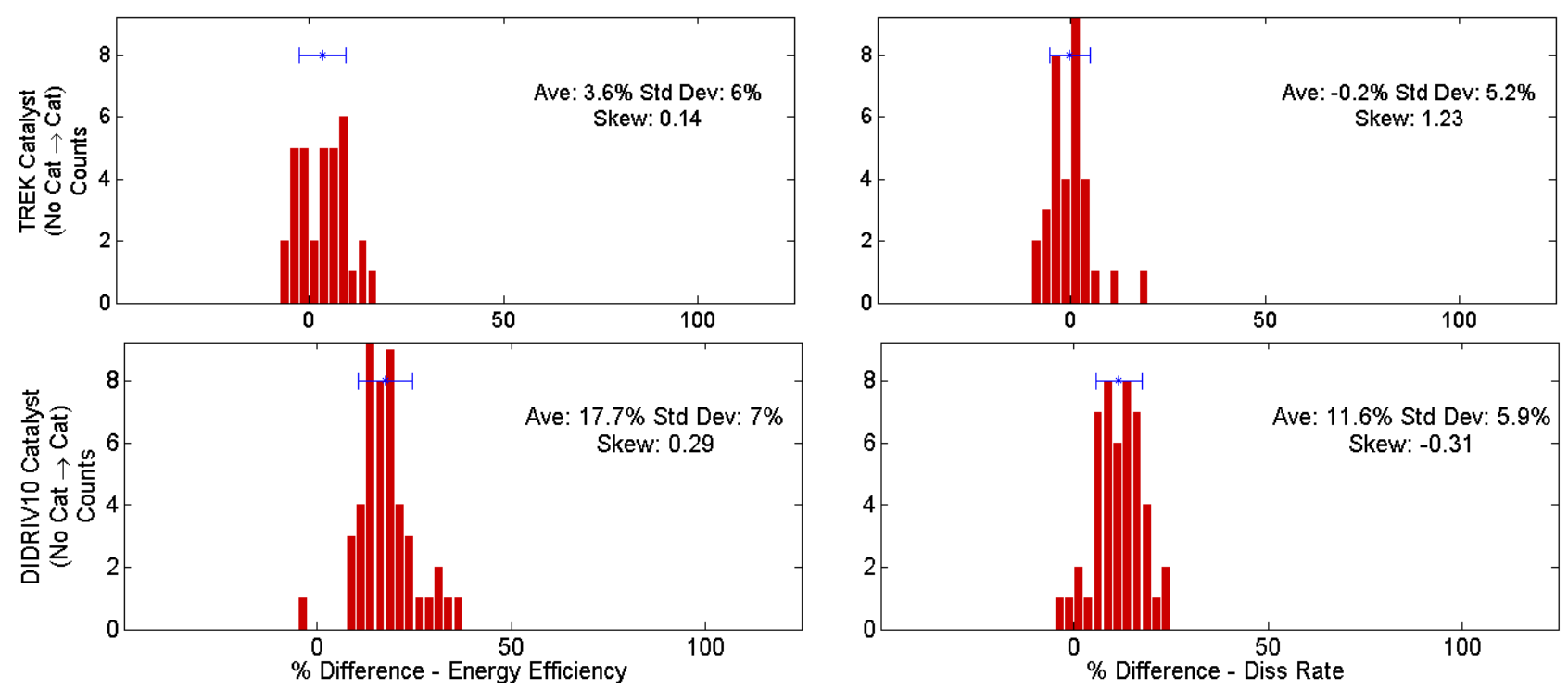

Figure 5.16: Percent difference (change) in energy efficiency and dissociation rate between experimental setups with and without the P25 catalyst in VADER.

The percent difference in energy efficiency and dissociation rate with the addition of a catalyst in VADER are in Figure 5.16. As expected, the TREK power supply showed a very small increase in the energy efficiency $(3.6 \% \pm 6 \%)$ and no change in the dissociation rate $(-0.2 \% \pm 3.1 \%)$ due to the catalyst. However, the addition of a new material into the discharge zone can effect the discharge dynamics of the plasma, which was monitored by looking at the change in forward power of the system. Figure 5.17 shows there is no change in forward power with the addition of the catalyst, therefore statistically there is no change in dissociation efficiency with the addition of a photocatalyst when using the TREK power supply. However, the average percent difference in energy efficiency does increase, but further tests would be needed to confirm this result.

The plots for the DIDRIV10 power supply show a significant increase in energy efficiency $(17.7 \% \pm 7 \%)$ and dissociation rate $(11.6 \% \pm 5.9 \%)$ with the addition of a catalyst. The 


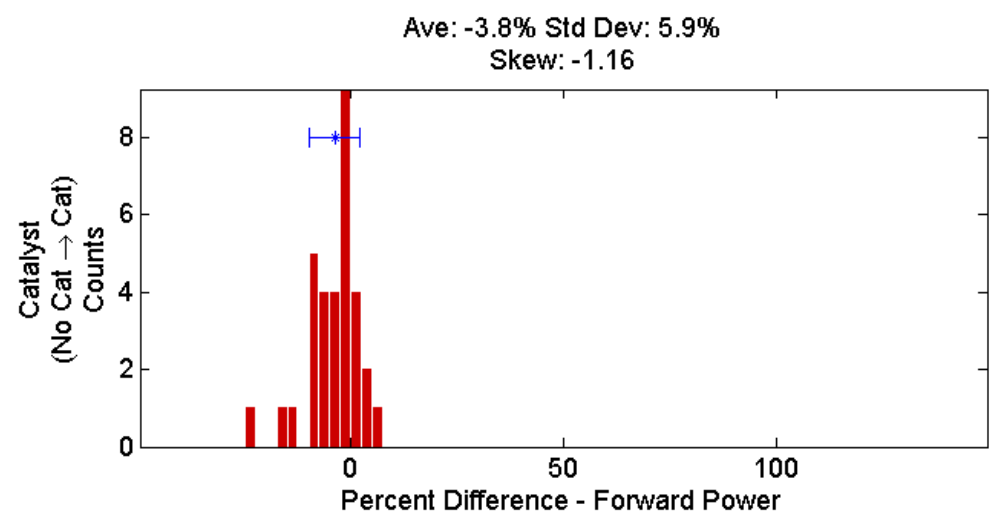

Figure 5.17: Percent difference in forward power due to the addition of $\mathrm{P} 25 \mathrm{TiO}_{2}$ for the TREK power supply driven plasmas.

increase in dissociation was accompanied by a small decrease in forward power, see Figure 5.18. A drop in forward power correlates with either a decrease or no change in the energy efficiency (see Figure 5.4), therefore the catalyst is responsible for the increases in energy efficiency and dissociation rate.

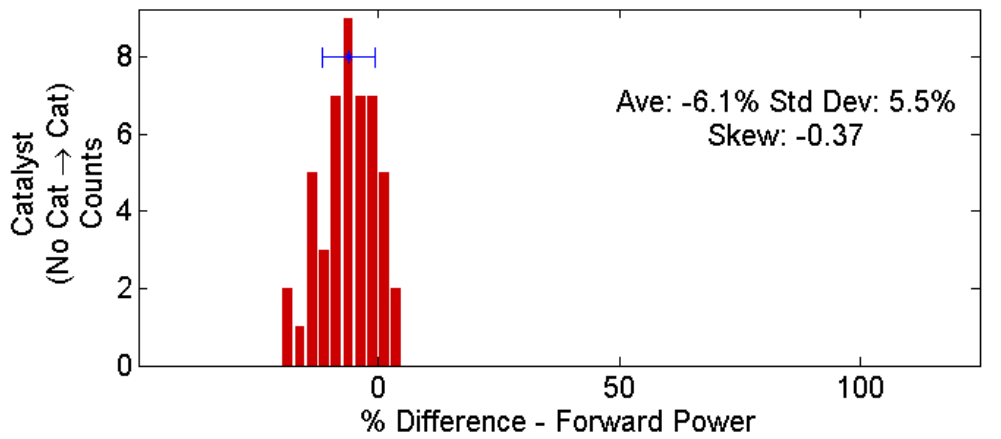

Figure 5.18: Percent difference in forward power due to the addition of $\mathrm{P} 25 \mathrm{TiO}_{2}$ for the DIDRIV10 power supply driven plasmas.

Despite similar setups, the DIDRIV10 power supply experiments showed that P25 can improve $\mathrm{CO}_{2}$ dissociation, while the TREK power supply experiments showed the catalyst has a minimal effect on dissociation. The main difference between power supplies is their driving frequency (discussed in Section 5.4.1). The driving frequency affects the catalyst's 
effectiveness multiple ways. For instance, higher frequencies increase the particle flux to the catalyst surface, due to the number of times a streamer cascades in a given amount of time. This increase in particle flux improves the chances of molecules reacting with the catalyst and increases the number of high energy electrons and ions colliding with the surface and exciting the catalyst to the energies needed for activation.

\subsubsection{Pulse Mode}

The adjustable pulse mode settings of the DIDRIV10 power supply were used to look at the effects of plasma startup on the energy efficiency and conversion rates in VADER. The three pulse modes tested were $2.4 \mathrm{~ms}$ on $2.4 \mathrm{~ms}$ off, $3.6 \mathrm{~ms}$ on $.5 \mathrm{~ms}$ off and continuous. Examples of the waveforms for the pulse modes are shown in Figure 3.6. At atmospheric pressures, it takes several milliseconds for ionized particles to recombine after the power supply is turned off. Therefore, charged particles in the $2.4 \mathrm{~ms}$ on $2.4 \mathrm{~ms}$ off case have more than enough time to recombine [66]. The different pulse modes were expected to either decrease in efficiency due to the higher voltages and powers required for plasma startup between duty cycles (as seen in Figure 3.6) or increase due to higher energy electrons created during the initial DBD cascade.

The experiments showed a minor increase in energy efficiency and a large drop in dissociation rate in pulsed plasmas, see Figure 5.19. The dissociation rates are directly related to the duty cycle of the pulses $\left(t_{o n} /\left(t_{o n}+t_{o f f}\right) \times 100\right)$. For example, the $2.4 \mathrm{~ms}$ on, $2.4 \mathrm{~ms}$ off (50\% duty cycle) dissociates approximately half the $\mathrm{CO}_{2}$ of the continuous mode, as seen in the graphs. A reduction in dissociation rate (therefore $\mathrm{CO}_{2}$ dissociation product densities) has been shown to increase the energy efficiency of the discharge, therefore it is unclear if the energy efficiency gains are due to the reduction in product density or pulse mode. However, 

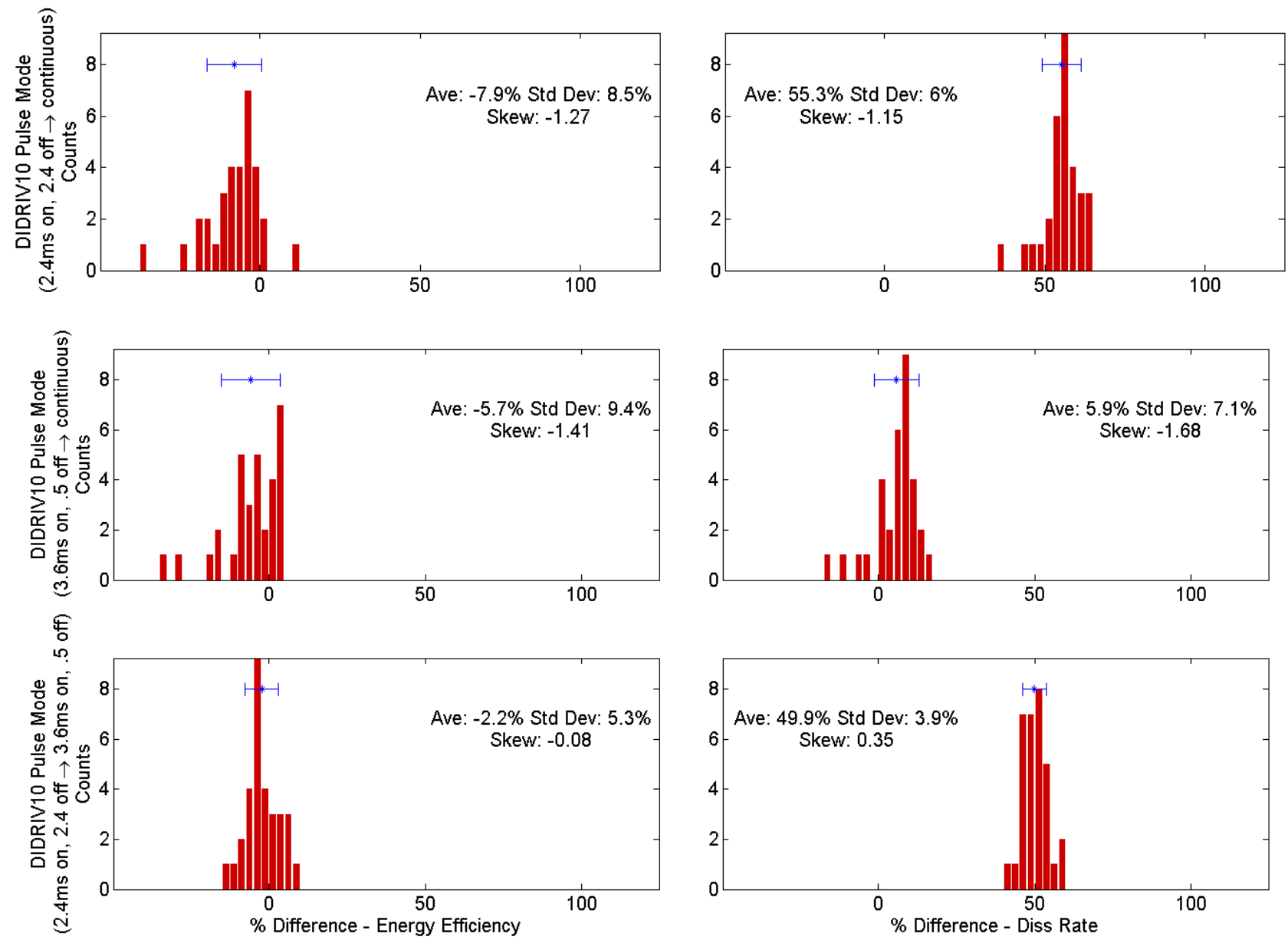

Figure 5.19: Percent difference (change) in energy efficiency and dissociation rate between pulse modes in VADER.

since the energy efficiency change by pulsing was expected to be relatively small and the change in energy efficiency was also small, the effects of $\mathrm{CO}_{2}$ products on dissociation in VADER must also be relatively small. 


\section{Chapter 6}

\section{Summary}

In this work, we have shown, using models and experiments, the important reactions and plasma dynamics that allow for $\mathrm{CO}_{2}$ dissociation within a DBD.

Control over the plasma properties is important for plasma chemistry, therefore the energy gain of particles within the electric field of a DBD was calculated. The results of the calculations were consistent with current DBD theory and showed that a DBD self-limits its plasma density and electron temperatures through a reduction in particle mean free path as both are increased. This work can be furthered by including the breakdown voltage of gases and the ionization potential of particles, which could be used to tailor the temperatures, densities and breakdown voltages of a DBD. Taking this analysis a step further, a PIC simulation of the particle dynamics could be used to better understand the discharge physics

for the same purposes. Initial tests using the OOPIC software package (by Tech-X, it has since been discontinued) were able to model the natural formation of multiple streamers on a 2D plane, therefore a more modern particle in cell (PIC) model should be able to fully model the DBD discharge in at least 2 dimensions. The OOPIC code was abandoned due to boundary layer and stability issues inherent in the code. 
To better understand the dynamics of the plasma chemistry a plasma chemical model was developed. The model went over the important reaction rates during each phase of the discharge and how they relate to the $\mathrm{CO}_{2}$ dissociative process, the results of this analysis showed that:

- $\mathrm{CO}_{2}$ dissociation is controlled by a combination of vibrational excitation, electron impact and recombination reactions.

- These reactions form carbon monoxide (CO) and atomic oxygen $(\mathrm{O})$ and mostly occur during the cascade phase of the streamer.

- The rates of these reactions are also highly dependent on electron temperature and the model suggests that a higher $\mathrm{CO}_{2}$ dissociation rate is achieved with higher electron energies.

- Once $\mathrm{CO}_{2}$ is dissociated into $\mathrm{CO}$ and $\mathrm{O}$, the relaxation of $\mathrm{O}$ during the afterglow/neutral phase determines the final chemical state of the gas.

- Relaxation of $\mathrm{O}$ (in a $\mathrm{CO}_{2}$ plasma) usually ends in the formation of $\mathrm{O}_{2}$, ozone or $\mathrm{CO}_{2}$.

- Even with the smallest fraction of $\mathrm{CO}$ and $\mathrm{O}_{2}(>0.001 \%$ of total gas), the plasma preferentially forms $\mathrm{O}_{2}$ through a reaction path following ozone formation and decay, which only occurs at a reasonable rate at gas temperatures below $500 \mathrm{~K}$.

- As more $\mathrm{CO}_{2}$ is dissociated in a DBD, the $\mathrm{CO}$ and $\mathrm{O}_{2}$ densities begin to influence the chemistry by absorbing large portions of electron energy that would otherwise go towards $\mathrm{CO}_{2}$ dissociation. 
- Therefore, converting large percentages of $\mathrm{CO}_{2}$ negatively influences the dissociation efficiency, as has been seen by other groups $[15,18,58]$.

- A possible reason for improved dissociation with the addition of argon into the plasma is the large charge exchange rates between $\mathrm{Ar}^{+}$and $\mathrm{CO}_{2}$.

- The charge exchange reaction creates $\mathrm{CO}_{2}^{+}$, which is then dissociated following $\mathrm{CO}_{2}^{+}$recombination.

Further improvements to the chemical model should entail temporal evolution of the discharge, expansion into further dimensions and the inclusion of more chemical reactions. Applying these effects would allow for a more rounded knowledge of the discharge physics and chemistry. Currently, Aerts et al. is developing a more advanced chemical model that takes into account vibrational excitation and excimer formation in a $\mathrm{CO}_{2} \mathrm{DBD}$ [36].

Several optical diagnostics were attempted in VADER, including molecular spectroscopy and Stark broadening spectroscopy. While they didn't provide all of the plasma parameters desired for these experiments, molecular spectroscopy did provide the first proof of $\mathrm{CO}_{2}$ dissociation within the DBD and the Stark broadening technique provided verification that the neutral gas temperature within the plasma is at room temperature. Improvements to these techniques should include an additive for the input gas to allow for Stark spectroscopy, such as hydrogen so that the well-documented hydrogen Balmer lines can be used to find the electron density. Also, the development of a collisional radiative model for a $\mathrm{CO}_{2}$ plasma in the optical range would be highly beneficial for research into $\mathrm{CO}_{2}$ dissociation both for finding the plasma temperatures and accurate electron densities (by applying an accurate temperature to Stark spectroscopy).

The first variable tested in VADER was the dielectric materials to determine which is 
the most efficient at creating a DBD plasma. The dielectric tests showed that:

- Dielectrics with a higher $\epsilon / \mathrm{d}(\epsilon$ is the dielectric constant, $\mathrm{d}$ is the material thickness) are more efficient at creating a streamer discharge.

- The efficiency improvement is due to the increased electric fields that a thinner and higher dielectric constant material applies to the air gap, which lowers the applied voltage needed for discharge.

- It also shows that power loss in a DBD is most likely due to a combination of internal resistance and dielectric heating.

- Plasma emissions were invariant to the dielectric material.

- This is because the power supply compensated for the change in $\epsilon / d$ and shows that the dielectric has no effect on the plasma properties as long as the same electric field is applied to the air gap.

Future work should consist of verifying these results using different emission lines, different dielectric materials and the inclusion of a resistor in series with the circuit. Also, the effects of changing the electrode separation were inconsistent, by comparing multiple emission lines and molecular line shapes with the change in gap spacing it can be determined whether the gap spacing changes any of the discharge parameters.

The VADER dissociation experiments reviewed the energy efficiency and dissociation rates of a DBD. The key results of this analysis were:

- Energy efficiency of $\mathrm{CO}_{2}$ dissociation in VADER was low, never exceeding $\sim 2.5 \% \pm$ $0.5 \%$. 
- The $\mathrm{CO}_{2}$ dissociation rates in VADER were modest with a maximum dissociation rate of $\sim 4 \times 10^{-6} \pm 0.5 \times 10^{-6} \mathrm{~mol} / \mathrm{sec}$ with a $\mathrm{CO}_{2}$ percent conversion of $\sim 5 \%$.

- The dissociation rate in VADER increased linearly with power

- The slope of the line was shown to increase as the breakdown voltage was reduced (change in gas composition) and the driving frequency was increased.

- Other groups have seen that this linear trend eventually plateaus as the density of product gases increase [18].

For a more generalized application of these results, the effects of gas flow rate, driving voltage, gas composition, frequency, the effects of adding a photocatalyst and the effects of pulsing the plasma was tested in VADER.

- The gas residence time (flow rate) on $\mathrm{CO}_{2}$ dissociation was found to have a small effect on dissociation and had a dependency on both frequency and gas composition.

- It is proposed that the frequency and gas composition dependency is due to a combination of dissociation product buildup in the plasma and the build up of molecular energy in the discharge.

- Increasing the driving voltage and adding argon into feed gas the was shown to significantly improve the $\mathrm{CO}_{2}$ dissociation efficiency and rate in VADER.

- It is proposed that these increases in efficiency are due to an increase in the plasma streamer densities.

- Based on other groups' work, these increases in efficiency are expected to eventually plateau and reduce as product densities in the plasma are increased. 
- There is a resonant driving frequency which corresponds to higher dissociation efficiencies.

- The resonant frequency was found to correlate with the breakdown ratio $\left(\frac{V_{\max }}{V_{B r}}\right)$, which is generally determined by the driving voltage and gas composition.

- It is proposed that the resonant frequency is due to a predator-prey relationship between the dissociation of $\mathrm{CO}_{2}$ and the formation of the product gases $(\mathrm{CO}$ and $\left.\mathrm{O}_{2}\right)$.

- This is the first time a photocatalyst has been shown to improve $\mathrm{CO}_{2}$ dissociation within a plasma.

- At driving frequencies of $28.4 \mathrm{kHz}$ and $30 \mathrm{kHz}$, the addition of a thin layer of $\mathrm{TiO}_{2}$ on the dielectric surface improved the $\mathrm{CO}_{2}$ dissociation efficiencies and rate by $\sim 17 \% \pm 7 \%$ and $11.6 \% \pm 5.9 \%$, respectively.

- At lower frequencies $(0.5-5 \mathrm{kHz})$, the catalyst was found to have no effect on dissociation.

- These results suggest that lower driving frequencies give too much time for the catalyst to relax between discharges, thus higher frequency discharges are required for the use of photocatalysts in a plasma.

- The pulse mode results revealed that the amount of product gases created within VADER had only minor influences on the $\mathrm{CO}_{2}$ dissociation rate and efficiency

- Therefore, a product gas population of several percent of the total gas does not highly effect the dissociation chemistry, but says nothing about higher percent conversions. 
Further research into $\mathrm{CO}_{2}$ dissociation using a DBD should expand on the results shown. These experiments should be carried out with more commercially viable DBD designs, for instance with a cylindrical geometry. A larger range of flow rates should be attempted and compared in order to verify the effects of the gas residence time on dissociation. A power supply with a larger and more stable range of frequencies, voltages and power is needed to find the relationship between resonance frequencies, voltages and gas compositions. It was shown that dissociation rates improve with a reduction in gas breakdown voltage, therefore it would be prudent to look at the effects of adding different gases in to a DBD, including further studies into converting $\mathrm{CO}_{2}$ into a value added chemical. Many groups have done tests using various structures, foams, etc. for incorporating different catalysts into their experiments-similar experiments using $\mathrm{TiO}_{2}$ should be attempted.

This work has analyzed the plasma chemistry of $\mathrm{CO}_{2}$ dissociation, looked into various atmospheric pressure diagnostics for a DBD and looked into many of the variables that effect $\mathrm{CO}_{2}$ dissociation efficiency in a DBD. The results of which have shown that there are many factors that influence the efficiency of $\mathrm{CO}_{2}$ dissociation using a DBD. Each of these factors would need to be optimized for the most efficient dissociation to occur, but the simplified chemistry used in this analysis hides a large portion of the system's inefficiencies. For instance, a commercial reactor would have many more chemical species in order to create a value-added chemical as the final product and, unless a major breakthrough in chemical pathways is found, it would significantly increase the complexity of the reactions. Any increase in the complexity of reactions is often accompanied by inefficiencies and a decrease in selectivity. Therefore, it is the author's opinion that using DBDs for the purposes of environmental $\mathrm{CO}_{2}$ reduction is not viable. Even so, this research is relevant to understanding the chemical processes that occur within DBDs, as well as other plasma sources. 


\section{Appendix A}

\section{Dielectric Material Analysis}

\section{A.1 First Dielectric Test}

The materials tested during the first dielectric tests included Macor (Table A.2), quartz (Table A.3) and boron nitride (Table A.4). The tests used 2" diameter electrodes, 0.0625" thick dielectrics that fully covered the electrode and a static pressure of 760 Torr with the prescribed gases. The dielectric was placed on VADER's ground electrode and all tests were done using the TREK power supply using a square wave form. The gas composition (Gas Comp.) is the percentage of input gas that is $\mathrm{CO}_{2}$ with the remaining gas consisting of argon. The electrodes were at a slight angle between each other, therefore the electrode separation (Elect. Sep.) was measured from the minimum distance between the electrode and dielectric. This non-uniformity in electrode distances led to discharges that preferentially formed and expanded from the shortest gap location (lopsided). The voltage, frequency and power were measured using the internal monitors of the TREK power supply. The plasma mode identifies the physical attributes of the plasma based on a visual inspection, the identified modes are listed in Table A.1. Max power identifies the power and frequency during which 
the highest forward powers were measured and usually corresponded with the most uniform and strongest emission from the plasma.

Table A.1: The plasma modes observed during the first dielectric tests

\begin{tabular}{lll}
\hline Plasma Mode & & Description \\
\hline No Plasma & N & No plasma formed \\
Diffuse Plasma & D & No visible streamers, looks similar to a glow discharge \\
Diffuse Streamers & DS & Like diffuse plasma, but multiple streamers visible within glow \\
Multiple Streamers & MS & Multiple streamers visible, glow discharge is dim or not visible \\
Single Streamer & SS & Single bright streamer, weaker streamers may form around cen- \\
& & tral streamer
\end{tabular}

\section{A.2 Second Dielectric Test}

The materials tested for the second set of dielectric tests were quartz $(0.0625$ " thick) and alumina (0.06"thick)-see Table A.5. All tests used the 2" diameter electrodes in air (760 Torr). The dielectric was placed on VADER's ground electrode and all tests were done using the DIDRIV10 power supply. As in the first dielectric tests, the electrodes were not parallel, therefore the electrode separation (Elect. Sep.) was measured from the closest two electrodes. The non-uniformity in electrode distances led to discharges that preferentially formed and expanded from the shortest gap location, therefore the amount of the electrode surface covered by the discharge was recorded (Disch. Vol.). The DBD streamers appeared to either move stochastically (random movement) or with a pulsing motion away from the shortest gap (pulsed movement), which was noted in the comments. The voltage, current were measured using the Rogowski coil and voltage divider as described in Section 3.2.3. For each experimental setting the $399.5 \mathrm{~nm}$ emission line was measured using the McPherson monochromator (described in Section 3.2.2) with a 20 second integration time. Each optical 
measurement was taken at the same position (focused at the center of the DBD) and the peak counts recorded. The peak counts were used to show if there is any relative change in electron density or electron temperature within the plasma. Also shown are the peak counts normalized to the measured power. 
Table A.2: Dielectric testing - Macor

\begin{tabular}{|c|c|c|c|c|c|c|c|c|}
\hline \multirow{2}{*}{$\begin{array}{l}\text { Gas } \\
\text { Comp. } \\
\% \mathrm{CO}_{2}\end{array}$} & \multirow{2}{*}{$\begin{array}{l}\text { Elect. } \\
\text { Sep. } \\
\text { in }\end{array}$} & \multirow{2}{*}{$\begin{array}{c}\text { Voltage } \\
\mathrm{kV}\end{array}$} & \multicolumn{2}{|c|}{$\begin{array}{l}\text { Frequency } \\
\text { Range }\end{array}$} & \multirow[t]{2}{*}{ Mode } & \multicolumn{2}{|c|}{$\begin{array}{l}\text { Max } \\
\text { Power }\end{array}$} & \multirow[t]{2}{*}{ Comments } \\
\hline & & & $\mathrm{kHz}$ & $\mathrm{kHz}$ & & W & $\mathrm{kHz}$ & \\
\hline \multirow[t]{13}{*}{$100 \%$} & 0.0625 & 20 & 0.1 & 2 & $\mathrm{D}$ & 46 & 2 & very uniform \\
\hline & & & 1.6 & 5.1 & DS & 50 & 2.3 & Lopsided at higher frequencies \\
\hline & & & 5.2 & 10 & $\mathrm{~N}$ & & & \\
\hline & & 18 & 0.1 & 2.6 & $\mathrm{D}$ & 45 & 2.6 & very uniform \\
\hline & & & 2.7 & 5.3 & DS & 45 & 2.7 & Lopsided at higher frequencies \\
\hline & & & 5.4 & 10 & $\mathrm{~N}$ & & & \\
\hline & & 16 & 0.1 & 2.6 & $\mathrm{D}$ & 38 & 2.6 & very uniform \\
\hline & & & 2.7 & 5.1 & DS & 42 & 2.9 & Lopsided at higher frequencies \\
\hline & & & 5.2 & 10 & $\mathrm{~N}$ & & & \\
\hline & & 14 & 0.1 & 5 & DS & 37 & 3.9 & covers $<70 \%$ of electrode \\
\hline & & & 5.1 & 10 & $\mathrm{~N}$ & & & \\
\hline & & 12 & 0.1 & 10 & $\mathrm{~N}$ & & & \\
\hline & 0.125 & 20 & 0.1 & 10 & $\mathrm{~N}$ & & & \\
\hline \multirow[t]{20}{*}{$90 \%$} & 0.0625 & 20 & 0.1 & 2 & $\mathrm{D}$ & 48 & 2 & very uniform \\
\hline & & & 2.1 & 5.8 & DS & 49 & 2.2 & Lopsided at higher frequencies \\
\hline & & & 5.9 & 10 & $\mathrm{~N}$ & & & \\
\hline & & 18 & 0.1 & 2.3 & $\mathrm{D}$ & 44 & 2.3 & very uniform \\
\hline & & & 2.4 & 6 & DS & 45 & 2.5 & Lopsided at higher frequencies \\
\hline & & & 6.1 & 10 & $\mathrm{~N}$ & & & \\
\hline & & 16 & 0.1 & 2.4 & $\mathrm{D}$ & 38 & 2.4 & very uniform \\
\hline & & & 2.5 & 6 & DS & 41 & 2.9 & Lopsided at higher frequencies \\
\hline & & & 6.1 & 10 & $\mathrm{~N}$ & & & \\
\hline & & 14 & 0.1 & 3 & $\mathrm{D}$ & 35 & 3 & very uniform \\
\hline & & & 3.1 & 6 & DS & 38 & 3.4 & Lopsided at higher frequencies \\
\hline & & & 6.1 & 10 & $\mathrm{~N}$ & & & \\
\hline & & 12 & 0.1 & 5.9 & DS & 33 & 4.4 & covers $<70 \%$ of electrode \\
\hline & & & 6 & 10 & $\mathrm{~N}$ & & & \\
\hline & & 10 & 0.1 & 10 & $\mathrm{~N}$ & & & $\begin{array}{l}\text { Unstable plasma at certain frequen- } \\
\text { cies }\end{array}$ \\
\hline & 0.125 & 20 & 0.1 & 2.8 & DS & 52 & 2.5 & very uniform \\
\hline & & & 2.9 & 3.4 & MS & 50 & 2.9 & Lopsided at higher frequencies \\
\hline & & & 3.5 & 10 & $\mathrm{~N}$ & & & \\
\hline & & 18 & 0.1 & 10 & $\mathrm{~N}$ & & & $\begin{array}{l}\text { no plasma, if seeded makes a MS } \\
\text { plasma }\end{array}$ \\
\hline & 0.1875 & 20 & 0.1 & 10 & $\mathrm{~N}$ & & & \\
\hline
\end{tabular}




\begin{tabular}{|c|c|c|c|c|c|c|c|c|}
\hline $\begin{array}{l}\text { Gas } \\
\text { Comp. } \\
\% \mathrm{CO}_{2}\end{array}$ & $\begin{array}{l}\text { Elect. } \\
\text { Sep. } \\
\text { in }\end{array}$ & $\begin{array}{c}\text { Voltage } \\
\mathrm{kV}\end{array}$ & \multicolumn{2}{|c|}{$\begin{array}{l}\text { Frequency } \\
\text { Range }\end{array}$} & Mode & \multicolumn{2}{|c|}{$\begin{array}{l}\text { Max } \\
\text { Power }\end{array}$} & Comments \\
\hline \multirow[t]{23}{*}{$80 \%$} & 0.0625 & 20 & 0.1 & 1.6 & $\mathrm{D}$ & 37 & 1.6 & very uniform \\
\hline & & & 1.7 & 5.7 & DS & 49 & 2.2 & Lopsided at higher frequencies \\
\hline & & & 5.8 & 10 & $\mathrm{~N}$ & & & \\
\hline & & 18 & 0.1 & 1.8 & $\mathrm{D}$ & 36 & 1.8 & very uniform \\
\hline & & & 1.9 & 6.1 & DS & 44 & 2.6 & Lopsided at higher frequencies \\
\hline & & & 6.2 & 10 & $\mathrm{~N}$ & & & \\
\hline & & 16 & 0.1 & 2.2 & $\mathrm{D}$ & 33 & 2.2 & very uniform \\
\hline & & & 2.3 & 6.1 & DS & 41 & 2.9 & Lopsided at higher frequencies \\
\hline & & & 6.2 & 10 & $\mathrm{~N}$ & & & \\
\hline & & 14 & 0.1 & 2.8 & $\mathrm{D}$ & 34 & 2.8 & very uniform \\
\hline & & & 2.9 & 5.9 & DS & 37 & 3.3 & Lopsided at higher frequencies \\
\hline & & & 6 & 10 & $\mathrm{~N}$ & & & \\
\hline & & 12 & 0.1 & 5.8 & DS & 33 & 4.4 & covers $<80 \%$ of electrode \\
\hline & & & 5.9 & 10 & $\mathrm{~N}$ & & & \\
\hline & & 10 & 0.1 & 10 & $\mathrm{~N}$ & & & \\
\hline & 0.125 & 20 & 0.1 & 3 & DS & 52 & 2.4 & very uniform \\
\hline & & & 3.1 & 3.6 & MS & 46 & 3.1 & Lopsided at higher frequencies \\
\hline & & & 3.7 & 10 & $\mathrm{~N}$ & & & \\
\hline & & 18 & 0.1 & 2.1 & DS & 36 & 2.1 & $\begin{array}{l}\text { fairly uniform, covers } \sim 90 \% \text { of elec- } \\
\text { trode surface }\end{array}$ \\
\hline & & & 2.2 & 3.4 & MS & 47 & 2.9 & Lopsided at higher frequencies \\
\hline & & & 3.5 & 10 & $\mathrm{~N}$ & & & \\
\hline & & 16 & 0.1 & 10 & $\mathrm{~N}$ & & & \\
\hline & 0.1875 & 20 & 0.1 & 10 & $\mathrm{~N}$ & & & \\
\hline
\end{tabular}




\begin{tabular}{|c|c|c|c|c|c|c|c|c|}
\hline $\begin{array}{l}\text { Gas } \\
\text { Comp. } \\
\% \mathrm{CO}_{2}\end{array}$ & $\begin{array}{l}\text { Elect. } \\
\text { Sep. } \\
\text { in }\end{array}$ & $\begin{array}{c}\text { Voltage } \\
\text { kV }\end{array}$ & \multicolumn{2}{|c|}{$\begin{array}{l}\text { Frequency } \\
\text { Range } \\
\mathrm{kHz} \mathrm{kHz}\end{array}$} & Mode & \multicolumn{2}{|c|}{$\begin{array}{l}\text { Max } \\
\text { Power }\end{array}$} & Comments \\
\hline \multirow[t]{25}{*}{$70 \%$} & 0.0625 & 20 & 0.1 & 1.5 & $\mathrm{D}$ & 37 & 1.5 & very uniform \\
\hline & & & 1.6 & 5.8 & DS & 49 & 2.1 & Lopsided at higher frequencies \\
\hline & & & 5.9 & 10 & $\mathrm{~N}$ & & & \\
\hline & & 18 & 0.1 & 1.6 & $\mathrm{D}$ & 30 & 1.6 & very uniform \\
\hline & & & 1.7 & 6.1 & $\mathrm{DS}$ & 45 & 2.4 & Lopsided at higher frequencies \\
\hline & & & 6.2 & 10 & $\mathrm{~N}$ & & & \\
\hline & & 16 & 0.1 & 2.2 & $\mathrm{D}$ & 34 & 2.2 & very uniform \\
\hline & & & 2.3 & 6 & $\mathrm{DS}$ & 41 & 2.8 & Lopsided at higher frequencies \\
\hline & & & 6.1 & 10 & $\mathrm{~N}$ & & & \\
\hline & & 14 & 0.1 & 2.6 & $\mathrm{D}$ & 31 & 2.6 & very uniform \\
\hline & & & 2.7 & 5.9 & DS & 38 & 3.4 & Lopsided at higher frequencies \\
\hline & & & 6 & 10 & $\mathrm{~N}$ & & & \\
\hline & & 12 & 0.1 & 5.9 & DS & 33 & 4.4 & covers $\sim 90 \%$ of electrode \\
\hline & & & 6 & 10 & $\mathrm{~N}$ & & & \\
\hline & & 10 & 0.1 & 10 & $\mathrm{~N}$ & & & \\
\hline & 0.125 & 20 & 0.1 & 1.6 & $\mathrm{D}$ & 37 & 1.6 & very uniform \\
\hline & & & 1.7 & 2.8 & DS & 53 & 2.4 & very uniform \\
\hline & & & 2.9 & 4 & MS & 48 & 2.9 & Lopsided at higher frequencies \\
\hline & & & 4.1 & 10 & $\mathrm{~N}$ & & & \\
\hline & & 18 & 0.1 & 1.8 & $\mathrm{D}$ & 34 & 1.8 & very uniform \\
\hline & & & 1.9 & 3.4 & $\mathrm{DS}$ & 48 & 2.8 & very uniform \\
\hline & & & 3.5 & 3.9 & MS & 42 & 3.5 & Lopsided at higher frequencies \\
\hline & & & 4 & 10 & $\mathrm{~N}$ & & & \\
\hline & & 16 & 0.1 & 10 & $\mathrm{~N}$ & & & \\
\hline & 0.1875 & 20 & 0.1 & 10 & $\mathrm{~N}$ & & & \\
\hline
\end{tabular}


Table A.3: Dielectric testing - Quartz

\begin{tabular}{|c|c|c|c|c|c|c|c|c|}
\hline $\begin{array}{l}\text { Gas } \\
\text { Comp } \\
\% \mathrm{CO}_{2}\end{array}$ & $\begin{array}{l}\text { Elect. } \\
\text { Sep. } \\
\text { in }\end{array}$ & $\begin{array}{c}\text { Voltage } \\
\text { kV }\end{array}$ & \multicolumn{2}{|c|}{$\begin{array}{l}\text { Frequency } \\
\text { Range }\end{array}$} & Mode & \multicolumn{2}{|c|}{$\begin{array}{l}\text { Max } \\
\text { Power }\end{array}$} & Comments \\
\hline \multirow[t]{21}{*}{$100 \%$} & 0.0625 & 20 & 0.1 & 3.8 & D & 50 & 2.4 & Very uniform \\
\hline & & & 3.9 & 5.9 & DS & 39 & 3.9 & Lopsided \\
\hline & & & 6 & 10 & $\mathrm{~N}$ & & & \\
\hline & & 18 & 0.1 & 4 & $\mathrm{D}$ & 47 & 2.8 & Very uniform \\
\hline & & & 4.1 & 5.9 & DS & 36 & 4.1 & Lopsided \\
\hline & & & 6 & 10 & $\mathrm{~N}$ & & & \\
\hline & & 16 & 0.1 & 3.9 & $\mathrm{D}$ & 41 & 2.9 & Very uniform \\
\hline & & & 4 & 6.1 & DS & 36 & 4 & Lopsided \\
\hline & & & 6.2 & 10 & $\mathrm{~N}$ & & & \\
\hline & & 14 & 0.1 & 2.8 & $\mathrm{D}$ & 31 & 2.8 & Very uniform \\
\hline & & & 2.9 & 6.1 & DS & 38 & 3.7 & Lopsided \\
\hline & & & 6.2 & 10 & $\mathrm{~N}$ & & & \\
\hline & & 12 & 0.1 & 6.1 & DS & 32 & 4.8 & Lopsided \\
\hline & & & 6.2 & 10 & $\mathrm{~N}$ & & & \\
\hline & & 10 & 0.1 & 10 & $\mathrm{~N}$ & & & Unstable plsama \\
\hline & 0.1250 & 20 & 0.1 & 3.7 & DS & 52 & 2.7 & Lopsided \\
\hline & & & 3.8 & 10 & $\mathrm{~N}$ & & & \\
\hline & & 18 & 0.1 & 3.6 & DS & 44 & 3.4 & Lopsided \\
\hline & & & 3.7 & 10 & $\mathrm{~N}$ & & & \\
\hline & & 16 & 0.1 & 10 & $\mathrm{~N}$ & & & \\
\hline & 0.1875 & 20 & 0.1 & 10 & $\mathrm{~N}$ & & & \\
\hline \multirow[t]{15}{*}{$90 \%$} & 0.0625 & 20 & 0.1 & 3.6 & $\mathrm{D}$ & 50 & 2.4 & very uniform \\
\hline & & & 3.7 & 5.8 & DS & 39 & 3.9 & Lopsided \\
\hline & & & 5.9 & 10 & $\mathrm{~N}$ & & & \\
\hline & & 18 & 0.1 & 3.9 & $\mathrm{D}$ & 45 & 2.8 & very uniform \\
\hline & & & 4 & 6 & DS & 37 & 4 & Lopsided \\
\hline & & & 6.1 & 10 & $\mathrm{~N}$ & & & \\
\hline & & 16 & 0.1 & 3.8 & $\mathrm{D}$ & 41 & 3.3 & very uniform \\
\hline & & & 3.9 & 6.1 & DS & 38 & 3.9 & Lopsided \\
\hline & & & 6.2 & 10 & $\mathrm{~N}$ & & & \\
\hline & & 14 & 0.1 & 2.4 & $\mathrm{D}$ & 27 & 2.4 & very uniform \\
\hline & & & 2.5 & 6.1 & DS & 37 & 3.9 & Lopsided at higher frequencies \\
\hline & & & 6.2 & 10 & $\mathrm{~N}$ & & & \\
\hline & & 12 & 0.1 & 5.9 & DS & 32 & 4.4 & Lopsided \\
\hline & & & 6 & 10 & $\mathrm{~N}$ & & & \\
\hline & & 10 & 0.1 & 10 & $\mathrm{~N}$ & & & Unstable plasma \\
\hline
\end{tabular}




\begin{tabular}{|c|c|c|c|c|c|c|c|c|}
\hline $\begin{array}{l}\text { Gas } \\
\text { Comp } \\
\% \mathrm{CO}_{2} \\
\end{array}$ & $\begin{array}{l}\text { Elect. } \\
\text { Sep. } \\
\text { in }\end{array}$ & $\begin{array}{c}\text { Voltage } \\
\mathrm{kV}\end{array}$ & \multicolumn{2}{|c|}{$\begin{array}{l}\text { Frequency } \\
\text { Range }\end{array}$} & Mode & \multicolumn{2}{|c|}{$\begin{array}{l}\text { Max } \\
\text { Power }\end{array}$} & Comments \\
\hline \multirow[t]{8}{*}{$90 \%$} & 0.125 & 20 & 0.1 & 2.8 & $\mathrm{D}$ & 51 & 2.8 & covers $\sim 90 \%$ of dielectric \\
\hline & & & 2.9 & 3.9 & DS & 51 & 2.9 & Lopsided at higher frequencies \\
\hline & & & 4 & 10 & $\mathrm{~N}$ & & & \\
\hline & & 18 & 0.1 & 2 & $\mathrm{D}$ & 30 & 2 & covers $\sim 50 \%$ of dielectric \\
\hline & & & 2.1 & 3.9 & DS & 44 & 3.2 & Lopsided \\
\hline & & & 4 & 10 & $\mathrm{~N}$ & & & \\
\hline & & 16 & 0.1 & 10 & $\mathrm{~N}$ & & & \\
\hline & 0.1875 & 20 & 0.1 & 10 & $\mathrm{~N}$ & & & \\
\hline \multirow[t]{25}{*}{$80 \%$} & 0.0625 & 20 & 0.1 & 2.8 & $\mathrm{D}$ & 49 & 2.3 & very uniform \\
\hline & & & 2.9 & 5.9 & DS & 44 & 2.9 & Lopsided at higher frequencies \\
\hline & & & 6 & 10 & $\mathrm{~N}$ & & & \\
\hline & & 18 & 0.1 & 3 & $\mathrm{D}$ & 44 & 2.7 & very uniform \\
\hline & & & 3.1 & 6.2 & DS & 41 & 3.1 & Lopsided at higher frequencies \\
\hline & & & 6.3 & 10 & $\mathrm{~N}$ & & & \\
\hline & & 16 & 0.1 & 3 & $\mathrm{D}$ & 40 & 3 & very uniform \\
\hline & & & 3.1 & 6.4 & DS & 40 & 3.1 & Lopsided at higher frequencies \\
\hline & & & 6.5 & 10 & $\mathrm{~N}$ & & & \\
\hline & & 14 & 0.1 & 3.1 & $\mathrm{D}$ & 31 & 3.1 & very uniform \\
\hline & & & 3.2 & 6.3 & DS & 36 & 3.5 & Lopsided at higher frequencies \\
\hline & & & 6.4 & 10 & $\mathrm{~N}$ & & & \\
\hline & & 12 & 0.1 & 5.9 & DS & 32 & 4.4 & covers $<80 \%$ of electrode \\
\hline & & & 6 & 10 & $\mathrm{~N}$ & & & \\
\hline & & 10 & 0.1 & 10 & $\mathrm{~N}$ & & & Unstable plasma \\
\hline & 0.125 & 20 & 0.1 & 1.8 & $\mathrm{D}$ & 40 & 1.8 & very uniform \\
\hline & & & 1.9 & 4 & DS & 50 & 2.4 & Lopsided at higher frequencies \\
\hline & & & 4.1 & 10 & $\mathrm{~N}$ & & & \\
\hline & & 18 & 0.1 & 1.4 & $\mathrm{D}$ & 25 & 1.4 & very uniform \\
\hline & & & 1.5 & 4 & DS & 46 & 2.9 & Lopsided at higher frequencies \\
\hline & & & 4.1 & 10 & $\mathrm{~N}$ & & & \\
\hline & & 16 & 0.1 & 3 & DS & 40 & 3.9 & covers $<50 \%$ of electrode \\
\hline & & & 3.1 & 10 & $\mathrm{~N}$ & & & \\
\hline & & 14 & 0.1 & 10 & $\mathrm{~N}$ & & & \\
\hline & 0.1875 & 20 & 0.1 & 10 & $\mathrm{~N}$ & & & \\
\hline
\end{tabular}




\begin{tabular}{|c|c|c|c|c|c|c|c|c|}
\hline $\begin{array}{l}\text { Gas } \\
\text { Comp. } \\
\% \mathrm{CO}_{2}\end{array}$ & $\begin{array}{l}\text { Elect. } \\
\text { Sep. } \\
\text { in }\end{array}$ & $\begin{array}{c}\text { Voltage } \\
\text { kV }\end{array}$ & \multicolumn{2}{|c|}{$\begin{array}{l}\text { Frequency } \\
\text { Range }\end{array}$} & Mode & \multicolumn{2}{|c|}{$\begin{array}{l}\text { Max } \\
\text { Power }\end{array}$} & Comments \\
\hline \multirow[t]{26}{*}{$70 \%$} & 0.0625 & 20 & 0.1 & 2.3 & $\mathrm{D}$ & 48 & 2.3 & very unifrom \\
\hline & & & 2.4 & 6.7 & DS & 48 & 2.4 & Lopsided at higher frequencies \\
\hline & & & 6.8 & 10 & $\mathrm{~N}$ & & & \\
\hline & & 18 & 0.1 & 2.7 & $\mathrm{D}$ & 43 & 2.5 & very uniform \\
\hline & & & 2.8 & 7.1 & DS & 43 & 2.8 & Lopsided at higher frequencies \\
\hline & & & 7.2 & 10 & $\mathrm{~N}$ & & & \\
\hline & & 16 & 0.1 & 3 & $\mathrm{D}$ & 40 & 3 & very uniform \\
\hline & & & 3.1 & 7.5 & DS & 40 & 3.1 & Lopsided at higher frequencies \\
\hline & & & 7.6 & 10 & $\mathrm{~N}$ & & & \\
\hline & & 14 & 0.1 & 3.3 & $\mathrm{D}$ & 36 & 3.3 & very uniform \\
\hline & & & 3.4 & 7.6 & DS & 36 & 3.4 & Lopsided at higher frequencies \\
\hline & & & 7.7 & 10 & $\mathrm{~N}$ & & & \\
\hline & & 12 & 0.1 & 4.1 & $\mathrm{D}$ & 32 & 3.9 & very uniform \\
\hline & & & 4.2 & 7.2 & DS & 32 & 4.4 & Lopsided at higher frequencies \\
\hline & & & 7.3 & 10 & $\mathrm{~N}$ & & & \\
\hline & & 10 & 0.1 & 7.3 & DS & 27 & 5.5 & Covers $<60 \%$ electrode \\
\hline & & & 7.4 & 10 & $\mathrm{~N}$ & & & \\
\hline & & 8 & 0.1 & 10 & $\mathrm{~N}$ & & & \\
\hline & 0.125 & 20 & 0.1 & 3.9 & DS & 51 & 2.4 & Lopsided at higher frequencies \\
\hline & & & 4 & 10 & $\mathrm{~N}$ & & & \\
\hline & & 18 & 0.1 & 4 & DS & 46 & 2.9 & Lopsided at higher frequencies \\
\hline & & & 4.1 & 10 & $\mathrm{~N}$ & & & \\
\hline & & 16 & 0.1 & 3.8 & DS & 42 & 3.4 & covers $<80 \%$ electrode \\
\hline & & & 3.9 & 10 & $\mathrm{~N}$ & & & \\
\hline & & 14 & 0.1 & 10 & $\mathrm{~N}$ & & & Unstable plasma \\
\hline & 0.1875 & 20 & 0.1 & 10 & $\mathrm{~N}$ & & & \\
\hline
\end{tabular}


Table A.4: Dielectric testing - Boron Nitride

\begin{tabular}{|c|c|c|c|c|c|c|c|c|}
\hline $\begin{array}{l}\text { Gas } \\
\text { Comp } \\
\% \mathrm{CO}_{2} \\
\end{array}$ & $\begin{array}{l}\text { Elect. } \\
\text { Sep. } \\
\text { in } \\
\end{array}$ & $\begin{array}{c}\text { Voltage } \\
\mathrm{kV}\end{array}$ & \multicolumn{2}{|c|}{$\begin{array}{l}\text { Frequency } \\
\text { Range } \\
\mathrm{kHz} \mathrm{kHz}\end{array}$} & Mode & \multicolumn{2}{|c|}{$\begin{array}{l}\text { Max } \\
\text { Power }\end{array}$} & Comments \\
\hline \multirow[t]{10}{*}{$100 \%$} & 0.0625 & 20 & 0.1 & 3 & $\mathrm{D}$ & 51 & 2.4 & very uniform \\
\hline & & & 3.1 & 4.3 & DS & 45 & 3.1 & Lopsided at high frequencies \\
\hline & & & 4.4 & 10 & $\mathrm{~N}$ & & & \\
\hline & & 18 & 0.1 & 2.7 & $\mathrm{D}$ & 45 & 2.7 & very uniform \\
\hline & & & 2.8 & 4.3 & DS & 47 & 2.9 & Lopsided at high frequencies \\
\hline & & & 4.4 & 10 & $\mathrm{~N}$ & & & \\
\hline & & 16 & 0.1 & 4.4 & DS & 41 & 3.4 & Covers $<80 \%$ of electrode \\
\hline & & & 4.5 & 10 & $\mathrm{~N}$ & & & \\
\hline & & 14 & 0.1 & 10 & $\mathrm{~N}$ & & & \\
\hline & 0.125 & 20 & 0.1 & 10 & $\mathrm{~N}$ & & & \\
\hline \multirow[t]{10}{*}{$90 \%$} & 0.0625 & 20 & 0.1 & 2 & $\mathrm{D}$ & 43 & 2 & very uniform \\
\hline & & & 2.1 & 4.2 & DS & 51 & 2.4 & Lopsided at high frequencies \\
\hline & & & 4.3 & 10 & $\mathrm{~N}$ & & & \\
\hline & & 18 & 0.1 & 2.4 & $\mathrm{D}$ & 41 & 2.4 & very uniform \\
\hline & & & 2.5 & 4.1 & DS & 46 & 2.8 & Lopsided at high frequencies \\
\hline & & & 4.2 & 10 & $\mathrm{~N}$ & & & \\
\hline & & 16 & 0.1 & 4.1 & DS & 40 & 3.4 & Covers $<50 \%$ of electrode \\
\hline & & & 4.2 & 10 & $\mathrm{~N}$ & & & \\
\hline & & 14 & 0.1 & 10 & $\mathrm{~N}$ & & & \\
\hline & 0.125 & 20 & 0.1 & 10 & $\mathrm{~N}$ & & & \\
\hline
\end{tabular}




\begin{tabular}{|c|c|c|c|c|c|c|c|c|}
\hline $\begin{array}{l}\text { Gas } \\
\text { Comp. } \\
\% \mathrm{CO}_{2} \\
\end{array}$ & $\begin{array}{l}\text { Elect. } \\
\text { Sep. } \\
\text { in }\end{array}$ & $\begin{array}{c}\text { Voltage } \\
\mathrm{kV}\end{array}$ & \multicolumn{2}{|c|}{$\begin{array}{l}\text { Frequency } \\
\text { Range }\end{array}$} & Mode & \multicolumn{2}{|c|}{$\begin{array}{l}\text { Max } \\
\text { Power }\end{array}$} & Comments \\
\hline \multirow{24}{*}{$80 \%$} & 0.0625 & 20 & 0.1 & 1.6 & $\mathrm{D}$ & 36 & 1.6 & very uniform \\
\hline & & & 1.7 & 6.1 & DS & 50 & 2.3 & Lopsided at high frequencies \\
\hline & & & 6.2 & 10 & $\mathrm{~N}$ & & & \\
\hline & & 18 & 0.1 & 2 & $\mathrm{D}$ & 37 & 2 & very uniform \\
\hline & & & 2.1 & 6.3 & DS & 44 & 2.4 & Lopsided at high frequencies \\
\hline & & & 6.4 & 10 & $\mathrm{~N}$ & & & \\
\hline & & 16 & 0.1 & 2.3 & $\mathrm{D}$ & 35 & 2.3 & very uniform \\
\hline & & & 2.4 & 6.3 & DS & 41 & 2.9 & Lopsided at high frequencies \\
\hline & & & 6.4 & 10 & $\mathrm{~N}$ & & & \\
\hline & & 14 & 0.1 & 2.4 & $\mathrm{D}$ & 28.3 & 2.4 & very uniform \\
\hline & & & 2.5 & 6.3 & DS & 37 & 3.4 & Lopsided at high frequencies \\
\hline & & & 6.4 & 10 & $\mathrm{~N}$ & & & \\
\hline & & 12 & 0.1 & 6.3 & DS & 32 & 4.4 & Covers $<90 \%$ of electrode \\
\hline & & & 6.4 & 10 & $\mathrm{~N}$ & & & \\
\hline & & 10 & 0.1 & 10 & $\mathrm{~N}$ & & & \\
\hline & 0.125 & 20 & 0.1 & 1.1 & $\mathrm{D}$ & 26 & 1.1 & very uniform \\
\hline & & & 1.2 & 3.1 & DS & 52 & 2.4 & very uniform \\
\hline & & & 3.2 & 4 & MS & 45 & 3.2 & Lopsided at high frequencies \\
\hline & & & 4.1 & 10 & $\mathrm{~N}$ & & & \\
\hline & & 18 & 0.1 & 2 & DS & 35 & 2 & very uniform \\
\hline & & & 2.1 & 4.1 & MS & 47 & 2.9 & Lopsided at high frequencies \\
\hline & & & 4.2 & 10 & $\mathrm{~N}$ & & & \\
\hline & & 16 & 0.1 & 10 & $\mathrm{~N}$ & & & \\
\hline & 0.1875 & 20 & 0.1 & 10 & $\mathrm{~N}$ & & & \\
\hline
\end{tabular}




\begin{tabular}{|c|c|c|c|c|c|c|c|c|}
\hline \multirow{2}{*}{$\begin{array}{l}\text { Gas } \\
\text { Comp. } \\
\% \mathrm{CO}_{2}\end{array}$} & \multirow{2}{*}{$\begin{array}{l}\text { Elect. } \\
\text { Sep. } \\
\text { in }\end{array}$} & \multirow{2}{*}{$\begin{array}{c}\text { Voltage } \\
\mathrm{kV}\end{array}$} & \multicolumn{2}{|c|}{$\begin{array}{l}\text { Frequency } \\
\text { Range }\end{array}$} & \multirow[t]{2}{*}{ Mode } & \multicolumn{2}{|c|}{$\begin{array}{l}\text { Max } \\
\text { Power }\end{array}$} & \multirow[t]{2}{*}{ Comments } \\
\hline & & & $\mathrm{kHz}$ & $\mathrm{kHz}$ & & $\mathrm{W}$ & $\mathrm{kHz}$ & \\
\hline \multirow[t]{34}{*}{$70 \%$} & 0.0625 & 20 & 0.1 & 1.2 & $\mathrm{D}$ & 30 & 1.2 & very uniform \\
\hline & & & 1.3 & 4.3 & DS & 49 & 2.3 & very uniform \\
\hline & & & 4.4 & 6.8 & MS & 33 & 4.4 & Lopsided at high frequencies \\
\hline & & & 6.9 & 10 & $\mathrm{~N}$ & & & \\
\hline & & 18 & 0.1 & 2 & $\mathrm{D}$ & 38 & 2 & very uniform \\
\hline & & & 2.1 & 4.1 & DS & 45 & 2.4 & very uniform \\
\hline & & & 4.2 & 6.8 & MS & 34 & 4.3 & Lopsided at high frequencies \\
\hline & & & 6.9 & 10 & $\mathrm{~N}$ & & & \\
\hline & & 16 & 0.1 & 2.7 & $\mathrm{D}$ & 39 & 2.7 & very uniform \\
\hline & & & 2.8 & 4 & DS & 41 & 2.9 & very uniform \\
\hline & & & 4.1 & 7 & MS & 34 & 4.1 & Lopsided at high frequencies \\
\hline & & & 7.1 & 10 & $\mathrm{~N}$ & & & \\
\hline & & 14 & 0.1 & 2.9 & $\mathrm{D}$ & 35 & 2.9 & very uniform \\
\hline & & & 3 & 4.1 & DS & 37 & 3.4 & very uniform \\
\hline & & & 4.2 & 7.2 & MS & 33 & 4.3 & Lopsided at high frequencies \\
\hline & & & 7.3 & 10 & $\mathrm{~N}$ & & & \\
\hline & & 12 & 0.1 & 2.5 & DS & 23 & 2.5 & very uniform \\
\hline & & & 2.6 & 7 & MS & 33 & 3.9 & Lopsided at high frequencies \\
\hline & & & 7.1 & 10 & $\mathrm{~N}$ & & & \\
\hline & & 10 & 0.1 & 2.6 & DS & 16 & 2.6 & Covers $<80 \%$ of electrode \\
\hline & & & 2.7 & 6.5 & MS & 28 & 5.4 & Covers $<60 \%$ of electrode \\
\hline & & & 6.6 & 10 & $\mathrm{~N}$ & & & \\
\hline & & 8 & 0.1 & 10 & $\mathrm{~N}$ & & & \\
\hline & 0.125 & 20 & 0.1 & 1 & $\mathrm{D}$ & 23.5 & 1 & very uniform \\
\hline & & & 1.1 & 3 & DS & 52 & 2.4 & very uniform \\
\hline & & & 3.1 & 4.3 & MS & 44 & 3.1 & Lopsided at high frequencies \\
\hline & & & 4.4 & 10 & $\mathrm{~N}$ & & & \\
\hline & & 18 & 0.1 & 2.4 & DS & 44 & 2.4 & very uniform \\
\hline & & & 2.5 & 4.4 & MS & 47 & 2.9 & Lopsided at high frequencies \\
\hline & & & 4.5 & 10 & $\mathrm{~N}$ & & & \\
\hline & & 16 & 0.1 & 4.2 & MS & 42 & 3.3 & very uniform \\
\hline & & & 4.3 & 10 & $\mathrm{~N}$ & & & \\
\hline & & 14 & 0.1 & 10 & $\mathrm{~N}$ & & & \\
\hline & 0.1875 & 20 & 0.1 & 10 & $\mathrm{~N}$ & & & \\
\hline
\end{tabular}


Table A.5: Dielectric Testing - Alumina and Quartz

\begin{tabular}{llllllllrlll}
\hline Material & $\begin{array}{l}\text { Elect. } \\
\text { Sep. }\end{array}$ & Voltage & Freq. & Power & $\begin{array}{l}\text { Max } \\
\text { Volt. }\end{array}$ & $\begin{array}{l}\text { Max } \\
\text { Cur. }\end{array}$ & 399.5 nm line & $\begin{array}{l}\text { Disch. Comments } \\
\text { Vol. }\end{array}$ & \\
& in & $\%$ & $\mathrm{kHz}$ & $\mathrm{W}$ & $\mathrm{V}$ & $\mathrm{mA}$ & $\mathrm{Cts}$ & $\mathrm{Cts} / \mathrm{W}$ & $\%$ & \\
\hline Alumina & $1 / 8 "$ & 90 & 28.4 & 60 & 760 & 189 & 6943 & 115.7 & $<50$ & random movement \\
Quartz & $1 / 8 "$ & 90 & 28.4 & 79 & 930 & 210 & 7224 & 91.4 & $<50$ & random movement \\
Alumina & $1 / 8 "$ & 100 & 28.4 & 73 & 814 & 213 & 9232 & 126.5 & 50 & random movement \\
Quartz & $1 / 8 "$ & 100 & 28.4 & 110 & 990 & 236 & 9268 & 84.3 & 50 & random movement \\
Alumina & $1 / 8 "$ & 100 & 30 & 55 & 656 & 200 & 7477 & 135.9 & 50 & pulsed movement \\
Quartz & $1 / 8 "$ & 100 & 30 & 73 & 812 & 222 & 7761 & 106.3 & $<50$ & pulsed movement \\
Alumina & $1 / 16 "$ & 90 & 28.4 & 36 & 510 & 165 & 13364 & 371.2 & 85 & pulsed movement \\
Quartz & $1 / 16 "$ & 90 & 28.4 & 54.5 & 676 & 185 & 14021 & 257.3 & 65 & pulsed movement \\
Alumina & $1 / 16 "$ & 100 & 28.4 & 47 & 570 & 189 & 17119 & 364.2 & 90 & pulsed movement \\
Quartz & $1 / 16 "$ & 100 & 28.4 & 72 & 746 & 213 & 17639 & 245.0 & 75 & pulsed movement \\
Alumina & $1 / 16 "$ & 100 & 30 & 33 & 450 & 178 & 14333 & 434.3 & 70 & pulsed movement \\
Quartz & $1 / 16 "$ & 100 & 30 & 41.5 & 564 & 177 & 12185 & 293.6 & 65 & pulsed movement \\
\hline
\end{tabular}




\section{Appendix B}

\section{Collisional Cross-Sections for $\mathrm{CO}_{2}$}

\section{Dissociation}

All of the cross-sections have units of $10^{-16} \mathrm{~cm}^{2}$. The cross-sections include:

- Total Xsec - The combined elastic, momentum, vibrational, electronic, dissociative and ionization electron collision cross-sections.

- Recomb - Dissociative recombination, $\mathrm{AB}^{+}+\mathrm{e} \rightarrow \mathrm{A}+\mathrm{B}$.

- Note $\mathrm{Ar}^{+}$recombination does not dissociate, it becomes electronically excited and emits photons during recombination.

- Attach - Dissociative attachment, $\mathrm{AB}+\mathrm{e} \rightarrow \mathrm{AB}^{-} \rightarrow \mathrm{A}+\mathrm{B}^{-}$

- e-Impact - Electron impact dissociation, $\mathrm{AB}+\mathrm{e} \rightarrow \mathrm{A}+\mathrm{B}+\mathrm{e}$

- Ion - Electron impact ionization, $\mathrm{AB}+\mathrm{e} \rightarrow \mathrm{AB}^{+}+2 \mathrm{e}$

- Diss Ion - Dissociative Ionization, $\mathrm{AB}+\mathrm{e} \rightarrow \mathrm{A}+\mathrm{B}^{+}+2 \mathrm{e}$ 
- Vib - Vibrational excitation, $\mathrm{AB}+\mathrm{e} \rightarrow \mathrm{AB}^{*}+\mathrm{e}, *$ is a vibrationally excited species.

- $\mathrm{CO}_{2} \mathrm{Vib}$ is the sum of symmetric-stretching, bending and antisymmetric stretching states as described by Itikawa [38].

$-\mathrm{O}_{2} \mathrm{Vib}$ is the sum of ground state $\mathrm{O}_{2}$ into its first, second and third vibrational states $\left(\mathrm{v}^{\prime}=1,2,3\right)$ and resonant vibrational states below $1 \mathrm{eV}$ as described by Itikawa [155]

- Elec - Electronic excitation, $\mathrm{AB}+\mathrm{e} \rightarrow \mathrm{AB}^{\star}+\mathrm{e}, \star$ is an electronically excited species

- Ch Exch - Charge Exchange, $\mathrm{A}^{+}+\mathrm{B} \rightarrow \mathrm{A}+\mathrm{B}^{+}$, this is an ion-neutral collision cross-section.

Most of the compiled cross-sections were found using the NIFS and Aladdin numerical databases $[156,157]$. Molecules within parentheses following a reaction denotes which product of the reaction is charged at the end of the reaction. If no parentheses follow a reaction that provides charged products, the final product charge were not denoted in the cross-section source. However, the molecule with the lower ionization potential will be charged more frequently at the end of the reaction. 
Table B.1: Electron collision and charge exchange cross-sections $\left(10^{-16} \mathrm{~cm}^{2}\right)$

\begin{tabular}{|c|c|c|c|c|c|c|}
\hline $\begin{array}{l}T_{e} \\
\mathrm{eV}\end{array}$ & $\begin{array}{r}\mathrm{CO}_{2} \text { Tot Xsec } \\
{[38,158]}\end{array}$ & $\mathrm{CO}_{2}^{+}$Recomb & $\begin{array}{r}\mathrm{CO}_{2} \text { Attach } \\
{[38,41,158]}\end{array}$ & $\begin{array}{r}\mathrm{CO}_{2} \text { e-Impact } \\
{[38,41,158]}\end{array}$ & $\begin{array}{r}\mathrm{CO}_{2} \text { Ion } \\
{[38,41,158]}\end{array}$ & $\begin{array}{r}\mathrm{CO}_{2} \text { Diss Ion }\left(\mathrm{CO}^{+}\right) \\
{[38,41,158]}\end{array}$ \\
\hline 0 & $5.0 \mathrm{E}+01$ & $6.0 \mathrm{E}+01$ & & & & \\
\hline 0.5 & $1.5 \mathrm{E}+01$ & $4.8 \mathrm{E}+00$ & & & & \\
\hline 1 & $8.0 \mathrm{E}+00$ & $3.4 \mathrm{E}+00$ & & & & \\
\hline 1.5 & - & $2.8 \mathrm{E}+00$ & & & & \\
\hline 2 & $6.0 \mathrm{E}+00$ & $2.4 \mathrm{E}+00$ & & & & \\
\hline 2.5 & - & $2.1 \mathrm{E}+00$ & & & & \\
\hline 3 & $9.0 \mathrm{E}+00$ & $1.9 \mathrm{E}+00$ & & & & \\
\hline 3.5 & - & $1.8 \mathrm{E}+00$ & $6.2 \mathrm{E}-05$ & & & \\
\hline 4 & $1.5 \mathrm{E}+01$ & $1.7 \mathrm{E}+00$ & $1.1 \mathrm{E}-03$ & & & \\
\hline 4.5 & - & $2.4 \mathrm{E}+00$ & $1.2 \mathrm{E}-03$ & & & \\
\hline 5 & $9.0 \mathrm{E}+00$ & $2.3 \mathrm{E}+00$ & $2.8 \mathrm{E}-04$ & & & \\
\hline 5.5 & - & $2.2 \mathrm{E}+00$ & $2.6 \mathrm{E}-05$ & & & \\
\hline 6 & $7.5 \mathrm{E}+00$ & $2.1 \mathrm{E}+00$ & $1.8 \mathrm{E}-05$ & & & \\
\hline 6.5 & - & $2.0 \mathrm{E}+00$ & $1.4 \mathrm{E}-04$ & & & \\
\hline 7 & $9.0 \mathrm{E}+00$ & $1.9 \mathrm{E}+00$ & $6.9 \mathrm{E}-04$ & & & \\
\hline 7.5 & - & $1.8 \mathrm{E}+00$ & $2.2 \mathrm{E}-03$ & & & \\
\hline 8 & $1.0 \mathrm{E}+01$ & $1.8 \mathrm{E}+00$ & $4.2 \mathrm{E}-03$ & & & \\
\hline 8.5 & - & $1.7 \mathrm{E}+00$ & $2.8 \mathrm{E}-03$ & & & \\
\hline 9 & $1.2 \mathrm{E}+00$ & $1.7 \mathrm{E}+00$ & $7.8 \mathrm{E}-04$ & & & \\
\hline 9.5 & - & $1.6 \mathrm{E}+00$ & $2.3 \mathrm{E}-04$ & & & \\
\hline 10 & $1.4 \mathrm{E}+00$ & $1.6 \mathrm{E}+00$ & $6.2 \mathrm{E}-05$ & & & \\
\hline 10.5 & - & $1.3 \mathrm{E}+00$ & & & & \\
\hline 11 & - & $1.3 \mathrm{E}+00$ & & & & \\
\hline 11.5 & - & $9.9 \mathrm{E}-01$ & & & & \\
\hline 12 & - & $9.7 \mathrm{E}-01$ & & $2.0 \mathrm{E}-02$ & & \\
\hline 12.5 & - & $9.5 \mathrm{E}-01$ & & - & & \\
\hline 13 & - & $7.0 \mathrm{E}-01$ & & - & & \\
\hline 13.5 & - & $6.9 \mathrm{E}-01$ & & - & & \\
\hline 14 & - & $6.8 \mathrm{E}-01$ & & $5.1 \mathrm{E}-02$ & & \\
\hline 14.5 & - & $6.6 \mathrm{E}-01$ & & - & $5.5 \mathrm{E}-02$ & \\
\hline 15 & - & $4.4 \mathrm{E}-01$ & & - & $9.7 \mathrm{E}-02$ & \\
\hline 15.5 & - & $3.9 \mathrm{E}-01$ & & - & $1.4 \mathrm{E}-01$ & \\
\hline 16 & - & $3.8 \mathrm{E}-01$ & & $8.2 \mathrm{E}-02$ & $1.7 \mathrm{E}-01$ & \\
\hline 16.5 & - & $3.7 \mathrm{E}-01$ & & - & $2.2 \mathrm{E}-01$ & \\
\hline 17 & - & $3.7 \mathrm{E}-01$ & & - & $2.6 \mathrm{E}-01$ & \\
\hline 17.5 & - & $3.6 \mathrm{E}-01$ & & - & $2.9 \mathrm{E}-01$ & \\
\hline 18 & - & $3.2 \mathrm{E}-01$ & & $1.0 \mathrm{E}-01$ & $3.3 \mathrm{E}-01$ & \\
\hline 18.5 & - & $3.1 \mathrm{E}-01$ & & - & $3.7 \mathrm{E}-01$ & \\
\hline 19 & - & $3.1 \mathrm{E}-01$ & & - & 4.3E-01 & \\
\hline 19.5 & - & $3.1 \mathrm{E}-01$ & & - & $4.5 \mathrm{E}-01$ & \\
\hline 20 & $1.7 \mathrm{E}+01$ & $3.0 \mathrm{E}-01$ & & $1.2 \mathrm{E}-01$ & $4.9 \mathrm{E}-01$ & \\
\hline 20.5 & - & $2.6 \mathrm{E}-01$ & & - & $5.4 \mathrm{E}-01$ & \\
\hline 21 & - & $2.6 \mathrm{E}-01$ & & - & $5.8 \mathrm{E}-01$ & \\
\hline 21.5 & - & $2.5 \mathrm{E}-01$ & & - & $6.2 \mathrm{E}-01$ & \\
\hline 22 & - & $2.5 \mathrm{E}-01$ & & - & $6.8 \mathrm{E}-01$ & \\
\hline 22.5 & - & $2.5 \mathrm{E}-01$ & & - & 7.3E-01 & \\
\hline 23 & - & $2.5 \mathrm{E}-01$ & & - & $7.8 \mathrm{E}-01$ & \\
\hline 23.5 & - & $2.4 \mathrm{E}-01$ & & - & 8.3E-01 & \\
\hline 24 & - & $2.1 \mathrm{E}-01$ & & $1.5 \mathrm{E}-01$ & $8.8 \mathrm{E}-01$ & \\
\hline 24.5 & - & $2.0 \mathrm{E}-01$ & & - & - & \\
\hline 25 & - & $2.0 \mathrm{E}-01$ & & - & $9.7 \mathrm{E}-01$ & $2.8 \mathrm{E}-02$ \\
\hline 25.5 & - & $2.0 \mathrm{E}-01$ & & - & - & - \\
\hline 26 & - & $2.0 \mathrm{E}-01$ & & - & - & - \\
\hline 26.5 & - & & & - & - & - \\
\hline 27 & - & & & - & - & - \\
\hline 27.5 & - & & & - & - & - \\
\hline 28 & - & & & $1.5 \mathrm{E}-01$ & - & - \\
\hline 28.5 & - & & & - & - & - \\
\hline 29 & - & & & - & - & - \\
\hline 29.5 & - & & & - & - & - \\
\hline 30 & $1.8 \mathrm{E}+01$ & & & $1.6 \mathrm{E}-01$ & $1.3 \mathrm{E}+00$ & $1.4 \mathrm{E}-01$ \\
\hline
\end{tabular}




\begin{tabular}{|c|c|c|c|c|c|c|}
\hline $\begin{array}{l}T_{e} \\
\mathrm{eV}\end{array}$ & $\begin{array}{r}\mathrm{CO}_{2} \text { Diss Ion }\left(\mathrm{O}^{+}\right) \\
{[38,41,158]}\end{array}$ & $\begin{array}{r}\mathrm{CO}_{2} \mathrm{Vib} \\
{[38,158]}\end{array}$ & $\begin{array}{r}O_{2} \text { Tot Xsec } \\
{[155,158]}\end{array}$ & $\begin{array}{r}O_{2}^{+} \text {Recomb } \\
{[83,84]}\end{array}$ & $\begin{array}{r}\mathrm{O}_{2} \text { Attach } \\
{[155,158]}\end{array}$ & $\begin{array}{r}\mathrm{O}_{2} \text { e-Impact } \\
{[155,158]}\end{array}$ \\
\hline 0 & & & $4.0 \mathrm{E}+00$ & $4.0 \mathrm{E}+02$ & & \\
\hline 0.5 & & & $5.0 \mathrm{E}+00$ & $6.0 \mathrm{E}+00$ & & \\
\hline 1 & & & $5.5 \mathrm{E}+00$ & $2.0 \mathrm{E}+00$ & & \\
\hline 1.5 & & $1.3 \mathrm{E}+00$ & - & $1.3 \mathrm{E}+00$ & & \\
\hline 2 & & $1.2 \mathrm{E}+00$ & $6.0 \mathrm{E}+00$ & $1.0 \mathrm{E}+00$ & & \\
\hline 2.5 & & $1.6 \mathrm{E}+00$ & - & 8.0E-01 & & \\
\hline 3 & & $2.0 \mathrm{E}+00$ & $6.5 \mathrm{E}+00$ & $5.0 \mathrm{E}-01$ & & \\
\hline 3.5 & & $3.2 \mathrm{E}+00$ & - & & & \\
\hline 4 & & $3.6 \mathrm{E}+00$ & $7.0 \mathrm{E}+00$ & $1.3 \mathrm{E}+00$ & & \\
\hline 4.5 & & $2.3 \mathrm{E}+00$ & - & & $4.4 \mathrm{E}-04$ & \\
\hline 5 & & $1.4 \mathrm{E}+00$ & $7.5 \mathrm{E}+00$ & & $2.2 \mathrm{E}-03$ & \\
\hline 5.5 & & $1.2 \mathrm{E}+00$ & - & & $6.3 \mathrm{E}-03$ & \\
\hline 6 & & $9.2 \mathrm{E}-01$ & $8.0 \mathrm{E}+00$ & & $1.1 \mathrm{E}-02$ & \\
\hline 6.5 & & - & - & & $1.4 \mathrm{E}-02$ & \\
\hline 7 & & - & - & & $1.2 \mathrm{E}-02$ & \\
\hline 7.5 & & - & - & & $8.2 \mathrm{E}-03$ & \\
\hline 8 & & - & - & & $4.5 \mathrm{E}-03$ & \\
\hline 8.5 & & - & - & & $2.0 \mathrm{E}-03$ & \\
\hline 9 & & - & - & & $8.8 \mathrm{E}-04$ & \\
\hline 9.5 & & - & - & & $4.4 \mathrm{E}-04$ & \\
\hline 10 & & - & $1.0 \mathrm{E}+01$ & & $3.5 \mathrm{E}-04$ & \\
\hline 10.5 & & - & - & & & \\
\hline 11 & & - & - & & & \\
\hline 11.5 & & - & - & & & \\
\hline 12 & & - & - & & & \\
\hline 12.5 & & - & - & & & \\
\hline 13 & & - & - & & & \\
\hline 13.5 & & - & - & & & $2.2 \mathrm{E}-01$ \\
\hline 14 & & - & - & & & - \\
\hline 14.5 & & - & - & & & - \\
\hline 15 & & $2.8 \mathrm{E}-01$ & - & & & - \\
\hline 15.5 & & - & - & & & - \\
\hline 16 & & - & - & & & - \\
\hline 16.5 & & - & - & & & - \\
\hline 17 & & - & - & & & - \\
\hline 17.5 & & - & - & & & - \\
\hline 18 & & - & - & & & - \\
\hline 18.5 & & - & - & & & 5.3E-01 \\
\hline 19 & & - & - & & & - \\
\hline 19.5 & & - & - & & & - \\
\hline 20 & & - & $1.1 \mathrm{E}+01$ & & & - \\
\hline 20.5 & & - & - & & & - \\
\hline 21 & & - & - & & & $5.7 \mathrm{E}-01$ \\
\hline 21.5 & & - & - & & & - \\
\hline 22 & & - & - & & & - \\
\hline 22.5 & & - & - & & & - \\
\hline 23 & & - & - & & & - \\
\hline 23.5 & & - & - & & & $5.3 \mathrm{E}-01$ \\
\hline 24 & & - & - & & & - \\
\hline 24.5 & & - & - & & & - \\
\hline 25 & $4.2 \mathrm{E}-02$ & - & - & & & - \\
\hline 25.5 & - & - & - & & & - \\
\hline 26 & - & - & - & & & - \\
\hline 26.5 & - & - & - & & & - \\
\hline 27 & - & - & - & & & - \\
\hline 27.5 & - & - & - & & & - \\
\hline 28 & - & - & - & & & - \\
\hline 28.5 & - & - & - & & & $5.9 \mathrm{E}-01$ \\
\hline 29 & - & - & - & & & - \\
\hline 29.5 & - & - & - & & & - \\
\hline 30 & $9.9 \mathrm{E}-02$ & $2.6 \mathrm{E}-01$ & $1.2 \mathrm{E}+01$ & & & $6.1 \mathrm{E}-01$ \\
\hline
\end{tabular}




\begin{tabular}{|c|c|c|c|c|c|c|}
\hline $\begin{array}{l}T_{e} \\
\mathrm{eV}\end{array}$ & $\begin{array}{r}\mathrm{O}_{2} \text { Ion } \\
{[155,158]}\end{array}$ & $\begin{array}{r}\mathrm{O}_{2} \text { Diss Ion } \\
{[155,158]}\end{array}$ & $\begin{array}{r}\mathrm{O}_{2} \mathrm{Vib} \\
{[155,158]}\end{array}$ & $\begin{array}{r}C O \text { Tot Xsec } \\
{[158]}\end{array}$ & 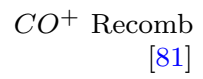 & $\begin{array}{r}C O \text { Attach } \\
{[158,159]}\end{array}$ \\
\hline 0 & & & & $7.0 \mathrm{E}+00$ & $1.0 \mathrm{E}+02$ & \\
\hline 0.5 & & & $1.0 \mathrm{E}+01$ & $1.3 \mathrm{E}+01$ & $1.0 \mathrm{E}+01$ & \\
\hline 1 & & & & $2.0 \mathrm{E}+01$ & $5.0 \mathrm{E}+00$ & \\
\hline 1.5 & & & & - & & \\
\hline 2 & & & & $6.0 \mathrm{E}+01$ & & \\
\hline 2.5 & & & & - & & \\
\hline 3 & & & & $3.5 \mathrm{E}+01$ & & \\
\hline 3.5 & & & & - & & \\
\hline 4 & & & & $2.0 \mathrm{E}+01$ & & \\
\hline 4.5 & & & & - & & \\
\hline 5 & & & $1.3 \mathrm{E}-01$ & $1.5 \mathrm{E}+01$ & & \\
\hline 5.5 & & & - & - & & \\
\hline 6 & & & - & $1.4 \mathrm{E}+01$ & & \\
\hline 6.5 & & & - & - & & \\
\hline 7 & & & 4.6E-01 & - & & \\
\hline 7.5 & & & - & - & & \\
\hline 8 & & & - & - & & \\
\hline 8.5 & & & - & - & & \\
\hline 9 & & & - & - & & \\
\hline 9.5 & & & - & - & & $3.1 \mathrm{E}-03$ \\
\hline 10 & & & $6.8 \mathrm{E}-01$ & $1.2 \mathrm{E}+01$ & & $7.2 \mathrm{E}+00$ \\
\hline 10.5 & & & - & - & & $5.5 \mathrm{E}+00$ \\
\hline 11 & & & - & - & & $3.1 \mathrm{E}+00$ \\
\hline 11.5 & & & - & - & & $1.4 \mathrm{E}+00$ \\
\hline 12 & & & - & - & & $6.0 \mathrm{E}-01$ \\
\hline 12.5 & & & - & - & & $2.8 \mathrm{E}-01$ \\
\hline 13 & $1.2 \mathrm{E}-02$ & & - & - & & $2.2 \mathrm{E}-01$ \\
\hline 13.5 & - & & - & - & & \\
\hline 14 & - & & - & - & & \\
\hline 14.5 & - & & - & - & & \\
\hline 15 & - & & 7.9E-02 & - & & \\
\hline 15.5 & 7.3E-02 & & & - & & \\
\hline 16 & - & & & - & & \\
\hline 16.5 & - & & & - & & \\
\hline 17 & - & & & - & & \\
\hline 17.5 & - & & & - & & \\
\hline 18 & 1.6E-01 & & & - & & \\
\hline 18.5 & - & & & - & & \\
\hline 19 & - & & & - & & \\
\hline 19.5 & - & & & - & & \\
\hline 20 & - & & & $1.5 \mathrm{E}+01$ & & \\
\hline 20.5 & - & & & - & & \\
\hline 21 & - & & & - & & \\
\hline 21.5 & - & & & - & & \\
\hline 22 & - & & & - & & \\
\hline 22.5 & - & & & - & & \\
\hline 23 & 3.7E-01 & $1.7 \mathrm{E}-02$ & & - & & \\
\hline 23.5 & - & - & & - & & \\
\hline 24 & - & - & & - & & \\
\hline 24.5 & - & - & & - & & \\
\hline 25 & - & - & & - & & \\
\hline 25.5 & - & - & & - & & \\
\hline 26 & - & - & & - & & \\
\hline 26.5 & - & - & & - & & \\
\hline 27 & - & - & & - & & \\
\hline 27.5 & - & - & & - & & \\
\hline 28 & $5.6 \mathrm{E}-01$ & $7.8 \mathrm{E}-02$ & & - & & \\
\hline 28.5 & - & - & & - & & \\
\hline 29 & - & - & & - & & \\
\hline 29.5 & - & - & & - & & \\
\hline 30 & $6.4 \mathrm{E}-01$ & $1.1 \mathrm{E}-01$ & & $1.3 \mathrm{E}+01$ & & \\
\hline
\end{tabular}




\begin{tabular}{|c|c|c|c|c|c|c|}
\hline $\begin{array}{l}T_{e} \\
\mathrm{eV}\end{array}$ & $\begin{array}{r}C O \text { e-Impact } \\
{[41,158,160]}\end{array}$ & $\begin{array}{l}C O \text { Ion } \\
{[41,158]}\end{array}$ & $\begin{array}{l}C O \text { Diss Ion } \\
{[41,158,160]}\end{array}$ & $\begin{array}{r}C O \mathrm{Vib} \\
{[158,161]}\end{array}$ & $\begin{array}{c}C O \text { Elec } \\
{[158,161]}\end{array}$ & $\begin{array}{r}A r^{+} \text {Recomb } \\
{[158,161]}\end{array}$ \\
\hline 0 & & & & & & \\
\hline 0.5 & & & & $2.8 \mathrm{E}-01$ & & \\
\hline 1 & & & & $5.8 \mathrm{E}-01$ & & $5.9 \mathrm{E}-03$ \\
\hline 1.5 & & & & $4.6 \mathrm{E}+00$ & & 8.3E-03 \\
\hline 2 & & & & $5.5 \mathrm{E}+00$ & & $9.5 \mathrm{E}-03$ \\
\hline 2.5 & & & & - & & $9.6 \mathrm{E}-03$ \\
\hline 3 & & & & $9.0 \mathrm{E}-01$ & & $9.2 \mathrm{E}-03$ \\
\hline 3.5 & & & & - & & 8.1E-03 \\
\hline 4 & & & & - & & 7.2E-03 \\
\hline 4.5 & & & & - & & $6.4 \mathrm{E}-03$ \\
\hline 5 & & & & $1.0 \mathrm{E}-01$ & & $5.7 \mathrm{E}-03$ \\
\hline 5.5 & & & & - & & $5.0 \mathrm{E}-03$ \\
\hline 6 & & & & - & & $4.5 \mathrm{E}-03$ \\
\hline 6.5 & & & & - & & $3.6 \mathrm{E}-03$ \\
\hline 7 & & & & - & & $3.2 \mathrm{E}-03$ \\
\hline 7.5 & & & & $2.0 \mathrm{E}-02$ & & - \\
\hline 8 & & & & - & & $2.7 \mathrm{E}-03$ \\
\hline 8.5 & & & & - & & - \\
\hline 9 & & & & - & & $2.4 \mathrm{E}-03$ \\
\hline 9.5 & & & & - & & - \\
\hline 10 & & & & 3.0E-02 & & $2.1 \mathrm{E}-03$ \\
\hline 10.5 & & & & - & & - \\
\hline 11 & & & & - & & - \\
\hline 11.5 & & & & - & & - \\
\hline 12 & & & & - & & - \\
\hline 12.5 & & & & - & & - \\
\hline 13 & & & & - & & - \\
\hline 13.5 & $1.1 \mathrm{E}-01$ & & & - & & - \\
\hline 14 & - & & & - & & - \\
\hline 14.5 & - & $2.7 \mathrm{E}-02$ & & - & & - \\
\hline 15 & - & $5.1 \mathrm{E}-02$ & & - & & $1.3 \mathrm{E}-03$ \\
\hline 15.5 & - & $7.7 \mathrm{E}-02$ & & - & & - \\
\hline 16 & - & $1.1 \mathrm{E}-01$ & & - & & - \\
\hline 16.5 & - & $1.4 \mathrm{E}-01$ & & - & & - \\
\hline 17 & - & $1.8 \mathrm{E}-01$ & & - & & - \\
\hline 17.5 & - & $2.1 \mathrm{E}-01$ & & - & & - \\
\hline 18 & - & $2.5 \mathrm{E}-01$ & & - & & - \\
\hline 18.5 & $2.8 \mathrm{E}-01$ & $3.0 \mathrm{E}-01$ & & - & & - \\
\hline 19 & - & $3.4 \mathrm{E}-01$ & & - & & - \\
\hline 19.5 & - & $3.9 \mathrm{E}-01$ & & - & & - \\
\hline 20 & - & $4.3 \mathrm{E}-01$ & & $1.0 \mathrm{E}-01$ & $1.2 \mathrm{E}+00$ & $7.5 \mathrm{E}-04$ \\
\hline 20.5 & - & 4.7E-01 & & & - & - \\
\hline 21 & $4.8 \mathrm{E}-01$ & $5.2 \mathrm{E}-01$ & & & - & - \\
\hline 21.5 & - & $5.6 \mathrm{E}-01$ & & & - & - \\
\hline 22 & - & $6.0 \mathrm{E}-01$ & & & - & - \\
\hline 22.5 & - & $6.4 \mathrm{E}-01$ & & & - & - \\
\hline 23 & - & $6.8 \mathrm{E}-01$ & & & - & - \\
\hline 23.5 & $5.2 \mathrm{E}-01$ & $7.2 \mathrm{E}-01$ & & & - & - \\
\hline 24 & - & $7.7 \mathrm{E}-01$ & & & - & - \\
\hline 24.5 & - & - & & & - & - \\
\hline 25 & - & $7.4 \mathrm{E}-01$ & $1.5 \mathrm{E}-02$ & & - & $5.7 \mathrm{E}-04$ \\
\hline 25.5 & - & - & - & & - & - \\
\hline 26 & - & - & - & & - & - \\
\hline 26.5 & - & - & - & & - & - \\
\hline 27 & - & - & - & & - & - \\
\hline 27.5 & - & - & - & & - & - \\
\hline 28 & - & - & - & & - & - \\
\hline 28.5 & $6.6 \mathrm{E}-01$ & - & - & & - & - \\
\hline 29 & 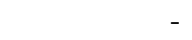 & - & - & & - & - \\
\hline 29.5 & - & - & - & & - & - \\
\hline 30 & $6.6 \mathrm{E}-01$ & $1.2 \mathrm{E}+00$ & 5.3E-02 & & $1.0 \mathrm{E}+00$ & $3.1 \mathrm{E}-04$ \\
\hline
\end{tabular}




\begin{tabular}{|c|c|c|c|c|c|c|}
\hline $\begin{array}{l}T_{e} \\
\mathrm{eV}\end{array}$ & $\begin{array}{r}A r \text { Ion } \\
{[162]}\end{array}$ & $\begin{array}{r}\text { Ar Elec } \\
{[161]}\end{array}$ & $\mathrm{CO}_{2}^{+}+\mathrm{O}_{2}$ Ch Exch & $\mathrm{Ar}^{+}+\mathrm{CO}_{2}$ Ch Exch & $\begin{array}{r}A r^{+}+C O \text { Ch Exch } \\
{[164]}\end{array}$ & 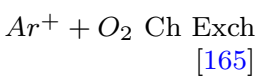 \\
\hline 0 & & & $2.9 \mathrm{E}+01$ & $5.5 \mathrm{E}+01$ & $9.0 \mathrm{E}+00$ & $1.3 \mathrm{E}+01$ \\
\hline 0.5 & & & $2.9 \mathrm{E}+01$ & $3.0 \mathrm{E}+01$ & $5.0 \mathrm{E}+00$ & $5.0 \mathrm{E}+00$ \\
\hline 1 & & & $2.9 \mathrm{E}+01$ & $1.5 \mathrm{E}+01$ & $3.8 \mathrm{E}+00$ & $3.5 \mathrm{E}+00$ \\
\hline 1.5 & & & $2.9 \mathrm{E}+01$ & $1.0 \mathrm{E}+01$ & - & - \\
\hline 2 & & & $2.9 \mathrm{E}+01$ & $7.0 \mathrm{E}+00$ & $1.5 \mathrm{E}+00$ & $2.5 \mathrm{E}+00$ \\
\hline 2.5 & & & $2.9 \mathrm{E}+01$ & - & - & \\
\hline 3 & & & $2.9 \mathrm{E}+01$ & $5.0 \mathrm{E}+00$ & $1.0 \mathrm{E}+00$ & $2.8 \mathrm{E}+00$ \\
\hline 3.5 & & & $2.9 \mathrm{E}+01$ & - & - & \\
\hline 4 & & & $2.9 \mathrm{E}+01$ & $3.0 \mathrm{E}+00$ & 8.0E-01 & $3.3 \mathrm{E}+00$ \\
\hline 4.5 & & & $2.9 \mathrm{E}+01$ & - & - & - \\
\hline 5 & & & $2.9 \mathrm{E}+01$ & $2.5 \mathrm{E}+00$ & $9.0 \mathrm{E}+00$ & $4.0 \mathrm{E}+00$ \\
\hline 5.5 & & & $2.9 \mathrm{E}+01$ & - & - & \\
\hline 6 & & & $2.9 \mathrm{E}+01$ & - & $1.0 \mathrm{E}+00$ & $4.5 \mathrm{E}+00$ \\
\hline 6.5 & & & $2.9 \mathrm{E}+01$ & - & - & - \\
\hline 7 & & & $2.9 \mathrm{E}+01$ & $1.5 \mathrm{E}+00$ & $1.3 \mathrm{E}+00$ & $4.5 \mathrm{E}+00$ \\
\hline 7.5 & & & $2.9 \mathrm{E}+01$ & - & - & - \\
\hline 8 & & & $2.9 \mathrm{E}+01$ & - & - & - \\
\hline 8.5 & & & $2.9 \mathrm{E}+01$ & - & - & - \\
\hline 9 & & & $2.9 \mathrm{E}+01$ & - & - & - \\
\hline 9.5 & & & $2.9 \mathrm{E}+01$ & - & - & - \\
\hline 10 & & & $2.9 \mathrm{E}+01$ & $1.0 \mathrm{E}+00$ & $1.6 \mathrm{E}+00$ & $4.3 \mathrm{E}+00$ \\
\hline 10.5 & & & $2.9 \mathrm{E}+01$ & - & - & - \\
\hline 11 & & & $2.9 \mathrm{E}+01$ & - & - & - \\
\hline 11.5 & & & $2.9 \mathrm{E}+01$ & - & - & - \\
\hline 12 & & & $2.9 \mathrm{E}+01$ & - & - & - \\
\hline 12.5 & & & $2.9 \mathrm{E}+01$ & - & - & - \\
\hline 13 & & & $2.9 \mathrm{E}+01$ & - & - & - \\
\hline 13.5 & & & $2.9 \mathrm{E}+01$ & - & - & - \\
\hline 14 & & & $2.9 \mathrm{E}+01$ & - & - & - \\
\hline 14.5 & & & $2.9 \mathrm{E}+01$ & - & - & - \\
\hline 15 & & & $2.9 \mathrm{E}+01$ & - & - & - \\
\hline 15.5 & & & $2.9 \mathrm{E}+01$ & - & - & - \\
\hline 16 & & $1.7 \mathrm{E}-01$ & $2.9 \mathrm{E}+01$ & - & - & - \\
\hline 16.5 & & - & $2.9 \mathrm{E}+01$ & - & - & - \\
\hline 17 & $1.6 \mathrm{E}-01$ & - & $2.9 \mathrm{E}+01$ & - & - & - \\
\hline 17.5 & - & - & $2.9 \mathrm{E}+01$ & - & - & - \\
\hline 18 & - & - & $2.9 \mathrm{E}+01$ & - & - & - \\
\hline 18.5 & $4.2 \mathrm{E}-01$ & - & $2.9 \mathrm{E}+01$ & - & - & - \\
\hline 19 & - & - & $2.9 \mathrm{E}+01$ & - & - & - \\
\hline 19.5 & - & - & $2.9 \mathrm{E}+01$ & - & - & - \\
\hline 20 & $6.0 \mathrm{E}-01$ & $5.7 \mathrm{E}-01$ & $2.9 \mathrm{E}+01$ & $2.5 \mathrm{E}+00$ & $4.0 \mathrm{E}+00$ & $4.0 \mathrm{E}+00$ \\
\hline 20.5 & - & - & $2.9 \mathrm{E}+01$ & - & - & - \\
\hline 21 & 7.7E-01 & - & $2.9 \mathrm{E}+01$ & - & - & - \\
\hline 21.5 & - & - & $2.9 \mathrm{E}+01$ & - & - & - \\
\hline 22 & - & - & $2.9 \mathrm{E}+01$ & - & - & - \\
\hline 22.5 & $1.0 \mathrm{E}+00$ & - & $2.9 \mathrm{E}+01$ & - & - & - \\
\hline 23 & - & - & $2.9 \mathrm{E}+01$ & - & - & - \\
\hline 23.5 & - & - & $2.9 \mathrm{E}+01$ & - & - & - \\
\hline 24 & - & - & $2.9 \mathrm{E}+01$ & - & - & - \\
\hline 24.5 & - & - & $2.9 \mathrm{E}+01$ & - & - & - \\
\hline 25 & $1.3 \mathrm{E}+00$ & - & $2.9 \mathrm{E}+01$ & - & - & - \\
\hline 25.5 & - & - & $2.9 \mathrm{E}+01$ & - & - & - \\
\hline 26 & - & - & $2.9 \mathrm{E}+01$ & - & - & - \\
\hline 26.5 & - & - & $2.9 \mathrm{E}+01$ & - & - & - \\
\hline 27 & - & - & $2.9 \mathrm{E}+01$ & - & - & - \\
\hline 27.5 & $1.6 \mathrm{E}+00$ & - & $2.9 \mathrm{E}+01$ & - & - & - \\
\hline 28 & - & - & $2.9 \mathrm{E}+01$ & - & - & - \\
\hline 28.5 & - & - & $2.9 \mathrm{E}+01$ & - & - & - \\
\hline 29 & - & - & $2.9 \mathrm{E}+01$ & - & - & - \\
\hline 29.5 & - & - & $2.9 \mathrm{E}+01$ & - & - & - \\
\hline 30 & $1.8 \mathrm{E}+00$ & 6.6E-01 & $2.9 \mathrm{E}+01$ & $2.8 \mathrm{E}+00$ & $3.7 \mathrm{E}+00$ & $4.5 \mathrm{E}+00$ \\
\hline
\end{tabular}




\section{Bibliography}

[1] 2013 Gobal Carbon Project, Electronic, 2013.

[2] Key World Energy Statistics, Technical report, International Energy Agency, 2013.

[3] S. Solomon, D. Qin, M. Manning, Z. Chen, M. Marquis, K. Avery, M. TigNOR, and H. Miller, editors, Climate Change 200\%: The Physical Science Basis, Cambridge University Press, 2007.

[4] M. Parry, O. Canziani, J. Palutikof, P. van Der Linden, and C. Hanson, editors, Climate Change 200\%: Impacts, Adaptation and Vulnerability, Cambridge University Press, 2007.

[5] M. R. Raupach, G. Marland, P. Ciais, C. Le Qur, J. G. Canadell, G. KlepPER, and C. B. Field, Proceedings of the National Academy of Sciences 104, 10288 (2007).

[6] US Greenhouse Gas Inventory Report, http://www.epa.gov/climatechange/ ghgemissions/usinventoryreport.html, 2011.

[7] EPA: Carbon Dioxide Capture and Sequestration, http://www.epa.gov/ climatechange/ccs/index.html. 
[8] L. L. Davies, K. Uchitel, and J. Ruple, Energy Policy 59, 745 (2013).

[9] P. B. Global CCS Institute, Accelerating the Uptake of CCS: Industrial use of Captured Carbon Dioxide, Technical report, The Global CCS Institute, 2011.

[10] P. Styring and D. Jansen, Carbon Capture and Utilisation in the Green Economy: Using $\mathrm{CO}_{2}$ to manufacture fuel, chemicals and materials, Technical report, Centre for Low Carbon Futures, 2011.

[11] U. Kogelschatz, Plasma Phys. Control. Fusion 46, B63 (2004).

[12] U. Kogelschatz, Contrib. Plasma Phys. 47, 80 (2007).

[13] C. Tendero, C. Tixier, P. Tristant, J. Desmaison, and P. Leprince, Spectrochimica Acta Part B: Atomic Spectroscopy 61, 2 (2006).

[14] A. Fridman, Plasma Chemistry, Cambridge University Press, 2008.

[15] S. Paulussen, B. Verheyde, X. Tu, C. D. Bie, T. Martens, D. Petrovic, A. Bogaerts, and B. Sels, Plasma Sources Science and Technology 19, 034015 (2010).

[16] A. Bill, A. Wokaun, B. Eliasson, E. Killer, and U. Kogelschatz, Energy Conversion and Management 38, Supplement, S415 (1997), Proceedings of the Third International Conference on Carbon Dioxide Removal.

[17] N. Shimba, S. Nakahara, and K. Itoyama, On decomposition of $\mathrm{CH}_{4}$ and $\mathrm{CO}_{2}$ by barrier discharge, in Pulsed Power Plasma Science, 2001. PPPS-2001. Digest of Technical Papers, volume 2, pp. 1122-1125 vol.2, 2001. 
[18] J.-Y. Wang, G.-G. Xia, A. Huang, S. L. Suib, Y. Hayashi, and H. Matsumoto, Journal of Catalysis 185, 152 (1999).

[19] Z. Yuhan, Study on CO2 reforming of $\mathrm{CH} 4$ by dielectric barrier discharge, in Fourth International Conference on Intelligent Computation Technology and Automation, edited by Z. YUHAN, number 874-877, 2011.

[20] V. J. Rico, J. L. Hueso, J. Cotrino, and A. R. Gonzalez-Elipe, The Journal of Physical Chemistry A 114, 4009 (2010), PMID: 20184329.

[21] M. Kraus, B. Eliasson, U. Kogelschatz, and A. Wokaun, Phys. Chem. Chem. Phys. 3, 294 (2001).

[22] Sakurai, Tsutomu, and A. Yokoyama, Journal of Nuclear Science and Technology 37, 814 (2000).

[23] L. F. Spencer and A. D. Gallimore, Plasma Sources Science and Technology 22, 015019 (2013).

[24] L. Spencer and A. Gallimore, Plasma Chemistry and Plasma Processing 31, 79 (2011).

[25] S. Y. Savinov, H. Lee, H. K. Song, and B.-K. NA, Industrial \& Engineering Chemistry Research 38, 2540 (1999).

[26] S. Mori, A. Tamamoto, and M. Suzuki, Plasma Sources Science and Technology 15, 609 (2006).

[27] Y. Nishimura and T. Takenouchi, Industrial \& Engineering Chemistry Fundamentals 15, 266 (1976). 
[28] A. Kobayashi and H. Hamanaka, Vacuum 80, 1294 (2006).

[29] S. Andreev, V. Zakharov, V. Ochkin, and S. Savinov, Spectrochimica Acta Part A 60, 3361 (2004).

[30] L. Bardos and H. Barankova, Vacuum 83, 522 (2009).

[31] J.-W. Park, D.-W. Kim, H.-S. Seon, K.-S. Kim, and D.-W. Park, Thin Solid Films 518, 4113 (2010).

[32] Y. Wen and X. Jiang, Plasma Chemistry and Plasma Processing 21, 665 (2001).

[33] V. D. Rusanov, A. A. Fridman, and G. V. Sholin, Soviet Physics Uspekhi 24, $447(1981)$.

[34] B. Darwent, Bond Dissociation Energies in Simple Molecules, NSRDS - data series 31, National Bureau of standards, 1970.

[35] M. Oehlschlager, D. Davidson, J. Jeffries, and R. Hanson, Z. Phys. Chem. $5,555(2005)$.

[36] R. Aerts, T. Martens, and A. Bogaerts, The Journal of Physical Chemistry C 116, 23257 (2012).

[37] P. Capezzuto, F. Cramarossa, R. D’Agostino, and E. Molinari, The Journal of Physical Chemistry 80, 882 (1976).

[38] Y. ItikawA, Journal of Physical and Chemical Reference Data 31, 749 (2002).

[39] C. Rond, A. Bultel, P. Boubert, and B. Cheron, Chemical Physics 354, 16 (2008). 
[40] L. Polak, Pure Appl. Chem. 39, 307 (1974).

[41] J. McConkey, C. Malone, P. Johnson, C. Winstead, V. McKoy, and I. KAnIK, Physics Reports 466, 1 (2008).

[42] NIST Chemistry WebBook, http://webbook.nist.gov/, 2011.

[43] L. G. Christophorou and J. A. D. Stockdale, The Journal of Chemical Physics 48, 1956 (1968).

[44] J. Van Durme, J. Dewulf, C. Leys, and H. Van Langenhove, APPLIED CATALYSIS B-ENVIRONMENTAL 78, 324 (2008).

[45] T. Inoue, A. Fujishima, S. Konishi, and K. Honda, Nature 277, 637 (1979).

[46] K. Rajalakshmi, Photocatalytic reduction of Carbon Dioxide in Conjunction with Decomposition of Water on Oxide Semiconductor Surfaces, Master's thesis, Indian Institute of Technology, Madras, 2011.

[47] S. PEKAREK, The European Physical Journal D 50, 171 (2008).

[48] S. PekareK, Journal of Physics D: Applied Physics 45, 075201 (2012).

[49] V. Havran, M. P. Dudukovic, and C. S. Lo, Industrial \& Engineering Chemistry Research 50, 7089 (2011).

[50] A. Fridman and L. Kennedy, Plasma Physics and Engineering, Taylor and Francis, 2004.

[51] J. L. Fox, Journal of Physics: Conference Series 4, 32 (2005), Sixth International Conference on Dissociative Recombination. 
[52] R. D. Thomas, Mass Spectrometry Reviews 27, 485 (2008).

[53] K. Seiersen, A. Al-Khalili, O. Heber, M. J. Jensen, I. B. Nielsen, H. B. Pedersen, C. P. Safvan, and L. H. Andersen, Phys. Rev. A 68, 022708 (2003).

[54] A. A. Viggiano, A. Ehlerding, F. Hellberg, R. D. Thomas, V. ZhaunerChyk, W. D. Geppert, H. Montaigne, M. Larsson, M. Kaminska, and F. Österdahl, The Journal of Chemical Physics 122, 226101 (2005).

[55] M. Tsuji, M. Nakamura, Y. Nishimura, and H. Obase, The Journal of Chemical Physics 108, 8031 (1998).

[56] G. J. Wilson, M. L. Turnidge, J. P. Reid, and C. J. S. M. Simpson, The Journal of Chemical Physics 102, 1192 (1995).

[57] M. Geoghegan, N. G. Adams, and D. Smith, Journal of Physics B: Atomic, Molecular and Optical Physics 24, 2589 (1991).

[58] G. Zheng, J. Jiang, Y. Wu, R. Zhang, and H. Hou, Plasma Chemistry and Plasma Processing 23, 59 (2003).

[59] S. L. Suib, S. L. Brock, M. Marquez, J. Luo, H. Matsumoto, and Y. Hayashi, The Journal of Physical Chemistry B 102, 9661 (1998).

[60] R. O. Denny, Plasma Dynamics, Clarendon Press, 1990.

[61] U. Kogelschatz, Fundamentals and Applications of Dielectric-Barrier Discharges, Paper, ABB Corporate Research Ltd., 2000.

[62] F. Massines, A. Rabehi, P. Decomps, R. B. Gadri, and P. Segur, Jounal of Applied Physics 83, 2950 (1998). 
[63] A. Schutze, J. Jeong, S. Babayan, J. Park, G. S. Selwyn, and R. F. Hicks, Plasma Science, IEEE Transactions on 26, 1685 (1998).

[64] L. B. Loeb and R. A. Wijsman, Journal of Applied Physics 19, 797 (1948).

[65] U. Kogelschatz, B. Eliasson, and W. Egli, Pure and Applied Chemistry 71, 1819 (1999).

[66] B. Eliasson, W. Egli, and U. Kogelschatz, Pure $\&$ Appl. Chem. 66, 1275 (1994).

[67] G. Steinle, D. Neundorf, W. Hiller, and M. Pietralla, Journal of Physics D: Applied Physics 32, 1350 (1999).

[68] R. Barni, P. Esena, and C. Riccardi, Journal of Applied Physics 97, 073301 (2005).

[69] J. Li and S. K. Dhali, Journal of Applied Physics 82, 4205 (1997).

[70] B. Eliasson, M. Hirth, and U. Kogelschatz, Journal of Physics D: Applied Physics 20, 1421 (1987).

[71] A. A. Kulikovsky, Phys. Rev. E 57, 7066 (1998).

[72] A. Chirokov, A. Gutsol, and A. Fridman, Pure Appl. Chem. 77, 487 (2005).

[73] U. Kogelschatz, Plasma Chemistry and Plasma Processing 23, 1 (2003).

[74] U. Kogelschatz, Plasma Science, IEEE Transactions on 30, 1400 (2002).

[75] P. M. Bellan, Fundamentals of Plasma Physics, Cambridge University Press, 2006.

[76] N. Y. Babaeva and M. J. Kushner, Plasma Sources Science and Technology 20, 035017 (2011). 
[77] M. LARoussi, IEEE Transactions on Plasma S 37, 714 (2009).

[78] R. Carman and R. Mildren, J. Phys. D: Apply. Phys. 33, L99 (2000).

[79] NIST Chemical Kinetics Database, http://kinetics.nist.gov/kinetics/index. jsp, 2013.

[80] R. Derai, P. R. Kemper, and M. T. Bowers, The Journal of Chemical Physics 82, 4517 (1985).

[81] S. Rosén, R. Peverall, M. Larsson, A. Le Padellec, J. Semaniak, A. Larson, C. Strömholm, W. J. van der Zande, H. Danared, and G. H. Dunn, Phys. Rev. A 57, 4462 (1998).

[82] K. Seiersen, O. Heber, M. J. Jensen, C. P. Safvan, and L. H. Andersen, The Journal of Chemical Physics 119, 839 (2003).

[83] C. H. Sheehan and J.-P. St.-Maurice, Journal of Geophysical Research: Space Physics 109 (2004).

[84] R. Peverall, S. Rosén, J. R. Peterson, M. Larsson, A. Al-Khalili, L. Vikor, J. Semaniak, R. Bobbenkamp, A. L. Padellec, A. N. MaurelLIS, and W. J. VAN DER ZANDE, The Journal of Chemical Physics 114, 6679 (2001).

[85] W. Tsang and R. F. Hampson, Journal of Physical and Chemical Reference Data 15, 1087 (1986).

[86] H. Hippler, R. Rahn, and J. Troe, The Journal of Chemical Physics 93, 6560 (1990). 
[87] J. Heimerl and T. Coffee, Combustion and Flame 35, 117 (1979).

[88] R. SHAw, International Journal of Chemical Kinetics 9, 929 (1977).

[89] R. Atkinson, D. L. Baulch, R. A. Cox, J. N. Crowley, R. F. Hampson, R. G. Hynes, M. E. Jenkin, M. J. Rossi, and J. Troe, Atmospheric Chemistry and Physics 4, 1461 (2004).

[90] S. Pshezhetskit, N. Morozov, S. Kamenetskaya, V. Siryatskaya, and E. Gribova, Russ. J. Phys. Chem. 33, 328 (1959).

[91] P. Harteck and S. Dondes, The Journal of Chemical Physics 26, 1734 (1957).

[92] S. Toвy, S. Sheth, and F. S. Toby, International Journal of Chemical Kinetics 16, 149 (1984).

[93] L. M. Arin and P. Warneck, The Journal of Physical Chemistry 76, 1514 (1972).

[94] H. J. MICK, M. BURMEISTER, and P. ROTH, AIAA Journal 31, 671 (1993).

[95] J. A. Blauer and G. R. Nickerson, A Survey of Vibrational Relaxation Rate Data for Processes Important to CO2-N2-H2O Infrared Plume Radiation, Technical report, Air Force Rocket Propulsion Laboratory, Ft. Belvoir, 1973.

[96] M. C. Gower and A. I. Carswell, Journal of Applied Physics 45, 3922 (1974).

[97] M. Huetz-Aubert and R. Tripodi, The Journal of Chemical Physics 55, 5724 (1971).

[98] J. A. Rutherford and D. A. Vroom, The Journal of Chemical Physics 64, 3057 (1976). 
[99] W. D. Geppert, D. Reignier, T. Stoecklin, C. Naulin, M. Costes, D. Chastaing, S. D. Le Picard, I. R. Sims, and I. W. M. Smith, Phys. Chem. Chem. Phys. 2, 2873 (2000).

[100] V. J. Rico, J. L. Hueso, J. Cotrino, V. Gallardo, B. Sarmiento, J. J. Brey, and A. R. Gonzalez-Elipe, Chem. Commun., 6192 (2009).

[101] Z. Salam, F. M., M. Amjad, and Z. Buntat, Power Electronics, IET 6, 1583 (2013).

[102] J. CARR, Laser Induced Fluorescence Studies of Electrostatic Double Layers in an Expanding Helicon Plasma, PhD thesis, West Virginia University (WVU), 2013.

[103] P. H. Krupenie, National Bureau of standards - National Standard Reference Data Series , 95 (1966), National Bureau of Standards.

[104] A. Keesee, Neutral Density Profiles in Argon Helicon Plasmas, PhD thesis, West Virginia University, 2006.

[105] C. Rond, P. Boubert, J.-M. Felio, and A. Chikhaoui, Journal of Thermophysics and Heat Transfer 21, 638 (2007).

[106] J. Annaloro, A. Bultel, and P. Omaly, Elaboration of a collisional-radiative model for CO2-N2-Ar plasma flows: Application to atmospheric Martian entries, in Fluid Dynamics and Co-located Conferences, pp. -, American Institute of Aeronautics and Astronautics, 2011, 42nd AIAA Thermophysics Conference.

[107] M. M. Kuraica and N. Konjevic, Applied Physics Letters 70, 1521 (1997). 
[108] R. Stamm, D. Boland, M. Christova, L. Godbert-Mouret, M. Koubiti, Y. Marandet, A. Mekkaoui, and J. Rosato, New Astronomy Reviews 53, 272 (2009), Proceedings of the $\{$ VII $\}$ Serbian Conference on Spectral Line Shapes (VII SCSLSA) held in Zrenjanin, Serbia June 15th-19th 2009.

[109] M. M. Kuraica, B. M. Obradovic, N. Cvetanovic, I. P. Dojcinovic, and J. Puric, New Astronomy Reviews 53, 266 (2009), Proceedings of the $\{\mathrm{VII}\}$ Serbian Conference on Spectral Line Shapes (VII SCSLSA) held in Zrenjanin, Serbia June 15th-19th 2009.

[110] A. Kramida and J. R. Fuhr, Atomic Spectral Line Broadening Bibliographic Database.

[111] J. Broekaert, Analytical Atomic Spectrometry with Flames and Plasmas, Wiley, 2006.

[112] A. Farmany, S. Abbasi, and A. Naghipour, Physics Letters B 650, 33 (2007).

[113] N. Konjevic, Physics Reports 316, 339 (1999).

[114] S. Duurovic and N. Konjevic, Plasma Sources Science and Technology 18, 035011 (2009).

[115] H. R. Griem, Plasma Spectroscopy, McGraw-Hill, 1964.

[116] A. Cox, Allen's Astrophysical Quantities, AIP Press, 2000.

[117] A. W. Ali and H. R. Griem, Phys. Rev. 140, A1044 (1965).

[118] H. GRIEm, Spectral line broadening by plasmas, Pure and Applied Physics Series, New York ; London : Academic Press, 1974. 
[119] R. Alvarez, A. Rodero, and M. Quintero, Spectrochimica Acta Part B: Atomic Spectroscopy 57, 1665 (2002).

[120] I. Hutchinson, Principles of Plasma Diagnostics, Cambridge University Press, 2005.

[121] N. Denisova, Plasma diagnostics using computed tomography method, in Plasma Science, 2008. ICOPS 2008. IEEE 35th International Conference on, pp. 1-1, 2008.

[122] Personal Correspondence with Dr. Hans Griem, 2011.

[123] Atomic Spectra database, http://physics.nist.gov/PhysRefData/ASD/index. html, 2011.

[124] A. Lesage, New Astronomy Reviews 52, 471 (2009).

[125] N. Konjevic, A. Lesage, J. R. Fuhr, and W. L. Wiese, Journal of Physical and Chemical Reference Data 31, 819 (2002).

[126] D. Nikolic, Z. Mijatovic, S. Duurovic, R. Kobilarov, and N. Konjevic, Journal of Quantitative Spectroscopy and Radiative Transfer 70, 67 (2001).

[127] C. Vidal, J. Cooper, and E. Smith, apjs 25, 37 (1973).

[128] N. Konjevic, M. Ivkovic, and N. Sakan, Spectrochimica Acta Part B: Atomic Spectroscopy 76, 16 (2012), Honoring Issue: A Collection of Papers on Plasma and Laser Spectrochemistry. Dedicated to Gary M. Hieftje on the occasion of his 70th birthday.

[129] H. Griem, Contributions to Plasma Physics 40, 46 (2000). 
[130] F. O. Thomas, T. C. Corke, M. Iqbal, A. Kozlov, and D. Schatzman, AiAA Journal 47, 2169 (2009).

[131] A. Meiners, M. Leck, and B. Abel, Review of Scientific Instruments 81, 113507 (2010).

[132] J. Cech, A. Brablec, P. Sahel, and M. Cernak, Czechoslovak Journal of Physics 56, B1074 (2006).

[133] Material properties, http://www.matweb.com/.

[134] Fused Silica (Quartz) properties, http://www.sciner.com/Opticsland/FS.htm.

[135] Kapton Properties, http://www2.dupont.com/Kapton/en_US/index.html.

[136] R. Li, Y. Yamaguchi, S. Yin, Q. Tang, and T. Sato, Solid State Ionics 172, 235 (2004), Proceedings of the Fifteenth International Symposium on the Reactivity of Solids.

[137] J. YaO, Z. T. Zhang, Z. Yu, S. J. Xu, Q. X. YU, and J. S. ZhaO, Journal of Physics: Conference Series 418, 012023 (2013).

[138] A. V. Pipa, J. Koskulics, R. Brandenburg, and T. Hoder, Review of Scientific Instruments 83, (2012).

[139] C. Gabriel, S. Gabriel, E. H. Grant, E. H. Grant, B. S. J. Halstead, and D. Michael P. Mingos, Chem. Soc. Rev. 27, 213 (1998).

[140] R. Bartnikas and E. J. McMahon, Engineering Dielectrics: Volume 1: Corona Measurement and Interpretation, ISTM, 1979. 
[141] E. DuARTE, InterNational Electrical Testing Association (NETA) , 1 (2004).

[142] G. Auday, P. Guillot, and J. Galy, Journal of Applied Physics 88, 4871 (2000).

[143] A. Dunaevsky, Y. Raitses, and N. J. Fisch, Physics of Plasmas 10, 2574 (2003).

[144] H. Akashi, T. Yoshinage, and A. Oda, Effect of secondary electron emission on atmospheric pressure oxygen dielectric barrier discharges, in ESCAMPIG XXI, 2012.

[145] Personal Correspondence with VTI employees, 2009.

[146] D. N. Joanes and C. A. Gill, Journal of the Royal Statistical Society: Series D (The Statistician) 47, 183 (1998).

[147] G. Bulmer, Principles of Statistics, Dover Books on Mathematics Series, Dover Publications, 1979.

[148] M. Aresta, A. Dibenedetto, and A. Angelini, Chemical Reviews ASAP (2013).

[149] U. Kogelschatz, Proc. SPIE 5483, 272 (2004).

[150] J. Guikema, N. Miller, J. Niehof, M. Klein, and M. Walhout, Phys. Rev. Lett. 85, 3817 (2000).

[151] Z. Liu, W. Wang, S. Zhang, D. Yang, L. Jia, and L. Dai, European Physical Journal D 66, 319 (2012).

[152] G. G. RAJu, Dielectrics and Electrical Insulation, IEEE Transactions on 11, 649 (2004).

[153] Y. Zhang, X.-H. Wen, and W.-H. Yang, Plasma Sources Science and Technology 16, $441(2007)$. 
[154] E. A. Sosnin, T. Oppenlander, and V. F. TArasenko, Journal of Photochemistry and Photobiology C: Photochemistry Reviews 7, 145 (2006).

[155] Y. ItIKAWA, Journal of Physical and Chemical Reference Data 38, 1 (2009).

[156] NIFS Database: Atomic \& Molecular Numerical Databases, http://dbshino.nifs . ac.jp/, 2013.

[157] Aladdin Numerical Database, http://www-amdis.iaea.org/ALADDIN/, 2013.

[158] K. Anzai, H. Kato, M. Hoshino, H. Tanaka, Y. Itikawa, L. Campbell, M. Brunger, S. Buckman, H. Cho, F. Blanco, G. Garcia, P. Limao-Vieira, and O. Ingolfsson, The European Physical Journal D 66, 1 (2012).

[159] D. RAPP and D. D. BRiglia, The Journal of Chemical Physics 43, 1480 (1965).

[160] G. J. Schulz, Phys. Rev. 128, 178 (1962).

[161] A. Zecca, G. Karwasz, and R. Brusa, La Rivista del Nuovo Cimento 19, 1 (1996).

[162] R. Rejoub, B. G. Lindsay, and R. F. Stebbings, Phys. Rev. A 65, 042713 (2002).

[163] G. D. Flesch and C. Y. NG, The Journal of Chemical Physics 97, 162 (1992).

[164] G. D. Flesch, S. Nourbakhsh, and C. Y. NG, The Journal of Chemical Physics 95, 3381 (1991).

[165] G. D. Flesch, S. Nourbakhsh, and C. Y. NG, The Journal of Chemical Physics 92, $3590(1990)$. 